\title{
OUTFLOWS AND BUBBLES IN TAURUS: STAR-FORMATION FEEDBACK SUFFICIENT TO MAINTAIN TURBULENCE
}

\author{
Huixian Li ${ }^{1,2,3}$, Di li ${ }^{1,2,4}$, Lei Qian ${ }^{1,2}$, Duo Xu ${ }^{1,3,5}$, Paul F. Goldsmith ${ }^{6}$, Alberto Noriega-Crespo ${ }^{7,8}$, Yuefang Wu ${ }^{9}$, \\ YUZHE SONG ${ }^{5}$, AND RENDONG NAN ${ }^{1,2}$ \\ ${ }^{1}$ National Astronomical Observatories, Chinese Academy of Sciences, Beijing 100012, China; 1hx@nao.cas.cn, dili@nao.cas.cn \\ ${ }^{2}$ Key Laboratory for Radio Astronomy, Chinese Academy of Sciences, Nanjing 210008, China \\ ${ }^{3}$ University of Chinese Academy of Sciences, Beijing 100049, China \\ ${ }_{5}^{4}$ Space Science Institute, Boulder, CO, USA \\ ${ }^{5}$ Nanjing University, Nanjing 210093, China \\ ${ }^{6}$ Jet Propulsion Laboratory, California Institute of Technology, Pasadena, CA 91109, USA \\ ${ }_{7}^{7}$ California Institute of Technology/IPAC, Pasadena, CA 91125, USA \\ ${ }^{8}$ Space Telescope Science Institute/JWST, Baltimore, MD 21218, USA \\ ${ }^{9}$ Department of Astronomy, Peking University, Beijing 100871, China \\ Received 2015 February 10; accepted 2015 May 11; published 2015 August 4
}

\begin{abstract}
We have identified outflows and bubbles in the Taurus molecular cloud based on the $\sim 100 \mathrm{deg}^{2}$ Five College Radio Astronomy Observatory ${ }^{12} \mathrm{CO}(1-0)$ and ${ }^{13} \mathrm{CO}(1-0)$ maps and the Spitzer young stellar object catalogs. In the main $44 \mathrm{deg}^{2}$ area of Taurus, we found 55 outflows, of which 31 were previously unknown. We also found 37 bubbles in the entire $100 \mathrm{deg}^{2}$ area of Taurus, none of which had been found previously. The total kinetic energy of the identified outflows is estimated to be $\sim 3.9 \times 10^{45} \mathrm{erg}$, which is $1 \%$ of the cloud turbulent energy. The total kinetic energy of the detected bubbles is estimated to be $\sim 9.2 \times 10^{46} \mathrm{erg}$, which is $29 \%$ of the turbulent energy of Taurus. The energy injection rate from the outflows is $\sim 1.3 \times 10^{33} \mathrm{erg} \mathrm{s}^{-1}$, which is $0.4-2$ times the dissipation rate of the cloud turbulence. The energy injection rate from bubbles is $\sim 6.4 \times 10^{33}$ $\mathrm{erg} \mathrm{s}^{-1}$, which is $2-10$ times the turbulent dissipation rate of the cloud. The gravitational binding energy of the cloud is $\sim 1.5 \times 10^{48} \mathrm{erg}$, that is, 385 and 16 times the energy of outflows and bubbles, respectively. We conclude that neither outflows nor bubbles can provide sufficient energy to balance the overall gravitational binding energy and the turbulent energy of Taurus. However, in the current epoch, stellar feedback is sufficient to maintain the observed turbulence in Taurus.
\end{abstract}

Key words: ISM: bubbles - ISM: individual objects (Taurus) - ISM: jets and outflows - ISM: kinematics and dynamics - surveys - turbulence

\section{INTRODUCTION}

During their early stage of evolution, stars experience a mass loss phase driven by strong stellar winds (Lada 1985). The stellar winds can entrain and accelerate ambient gas and inject momentum and energy into the surrounding environment, thereby significantly affect the dynamics and structure of their parent molecular clouds (Narayanan et al. 2008; Arce et al. 2011). Both outflows and bubbles are manifestations of strong stellar winds dispersing the surrounding gas. In general, collimated jet-like winds from young embedded protostars usually drive powerful collimated outflows, while wide-angle or spherical winds from pre-main-sequence stars are more likely to drive less-collimated outflows or bubbles (Arce et al. 2011). A bubble is a partially or fully enclosed threedimensional structure whose projection is a partial or full ring (Churchwell et al. 2006).

The kinetic energy of an outflow is very large $\left(10^{43}-10^{48}\right.$ erg; Lada 1985; Bachiller 1996), implying a substantial input of mechanical energy into its parent molecular cloud (Solomon et al. 1981). Feedback from young stars has been proposed as a significant aspect of the self-regulation of star formation (Norman \& Silk 1980; Franco 1983). Feedback may maintain the observed turbulence in molecular clouds and may also be responsible for stabilizing the clouds against gravitational collapse (Shu et al. 1987). The impact of outflows on the surrounding gas has primarily been studied in small regions such as Orion KL (Kwan \& Scoville 1976), L1551 (Snell et al. 1980), and GL 490 (Lada \& Harvey 1981) on scales of less than $10^{\prime}$. Recently, there have been a few studies related to outflow feedback in nearby clouds. Arce et al. (2010) undertook a complete survey of outflows in Perseus and found that outflows have an important impact on the environment immediately surrounding localized regions of active star formation, but that outflows have insufficient energy to feed the observed turbulence in the entire Perseus complex. Nakamura et al. (2011a, 2011b) studied the outflows in the $\rho$ Ophiuchi main cloud and Serpens south, and concluded that outflows can power the supersonic turbulence in their parent molecular cloud but do not have sufficient momentum to support the entire cloud against global gravitational contraction. Narayanan et al. (2012) identified 20 outflows in the Taurus region and concluded that outflows cannot sustain the observed turbulence seen in the entire cloud. In this paper, we report a systematic and detailed search for outflows around sources from the Spitzer Space Telescope (hereafter Spitzer) young stellar object (YSO) catalog and then estimate their impact on the entire Taurus molecular cloud.

Similar to outflows, bubbles are important morphological features in the star-formation process which can provide information about spherical stellar winds and the physical properties of their surrounding environments (Churchwell et al. 2006). Parsec-scale bubbles are usually found in massive 
star-forming regions (Heyer et al. 1992; Churchwell et al. 2006, 2007; Beaumont \& Williams 2010; Deharveng et al. 2010). The conventional thought has been that high-mass stars can drive spherical winds and easily create the observed bubbles, while the spherical winds from low- and intermediatemass stars are too weak to produce bubbles. However, Arce et al. (2011) studied shells (bubbles) in Perseus, a nearby lowmass star-forming molecular cloud, and concluded that the total energy input from outflows and shells is sufficient to maintain the turbulence.

The Taurus molecular cloud is at a distance of $140 \mathrm{pc}$ (Torres et al. 2009). It covers an area of more than $100 \mathrm{deg}^{2}$ (Ungerechts \& Thaddeus 1987). Using the $J=2-1$ line of ${ }^{12} \mathrm{CO}, 13$ outflows have been found around low-mass embedded YSOs in Taurus (Bontemps et al. 1996). There are 13 high-velocity molecular outflows in Taurus included in the catalog of Wu et al. (2004). Using JCMT-HARP ${ }^{12} \mathrm{CO} J=3-2$ observations, 16 outflows have been found in L1495, a "bowlshaped" region in the north-west corner of Taurus (Davis et al. 2010). Recently, 20 outflows have been identified, 8 of which were new detections with the Five College Radio Astronomy Observatory (FCRAO) ${ }^{12} \mathrm{CO} J=1-0$ and ${ }^{13} \mathrm{CO} J$ $=1-0$ data cubes covering the entire Taurus molecular cloud (Narayanan et al. 2012). The up-to-date catalog of YSOs (Rebull et al. 2010) from Spitzer provides us with an opportunity to search for outflows and bubbles in a more comprehensive manner. Here, we present a systematic and detailed search for outflows and bubbles in the vicinity of YSOs and estimate their impact on the overall Taurus molecular cloud.

The paper is organized as follows. In Section 2, we describe the data used in the study. The details, including searching methods, morphology, and physical parameters of outflows and bubbles, are presented in Sections 3 and 4, respectively. The driving sources of outflows and bubbles, their energy feedback to the parent cloud, and the potential sources of turbulent motions in Taurus are discussed in Section 5. In Section 6, we summarize the main results.

\section{THE DATA}

In our study, we used the ${ }^{12} \mathrm{CO}(1-0)$ and ${ }^{13} \mathrm{CO}(1-0)$ data observed with the $13.7 \mathrm{~m}$ FCRAO telescope (Narayanan et al. 2008). We also adopted the up-to-date catalog of Spitzer YSOs where 215 YSOs and 140 new YSO candidates in Taurus are reported (Rebull et al. 2010).

\subsection{FCRAO CO Maps}

The FCRAO CO survey was taken between 2003 and 2005 . The ${ }^{12} \mathrm{CO}$ and ${ }^{13} \mathrm{CO}$ maps are centered at $\alpha=04^{\mathrm{h}} 32^{\mathrm{m}} 44^{\mathrm{s}} .6$, $\delta=24^{\circ} 25^{\prime} 44^{\prime \prime} 6$ (J2000) covering an area of approximately $100 \mathrm{deg}^{2}$. The FWHM beam width is $45^{\prime \prime}$ for ${ }^{12} \mathrm{CO}$ and $47^{\prime \prime}$ for ${ }^{13} \mathrm{CO}$. The pixel size of the resampled data is $20^{\prime \prime}$, which corresponds to $0.014 \mathrm{pc}$ at a distance of $140 \mathrm{pc}$. There are 80 channels for ${ }^{12} \mathrm{CO}$ and 76 channels for ${ }^{13} \mathrm{CO}$, covering approximately -5 to $+14.9 \mathrm{~km} \mathrm{~s}^{-1}$. The width of a velocity channel is $0.254 \mathrm{~km} \mathrm{~s}^{-1}$ for ${ }^{12} \mathrm{CO}$ and $0.266 \mathrm{~km} \mathrm{~s}^{-1}$ for ${ }^{13} \mathrm{CO}$ (Goldsmith et al. 2008; Narayanan et al. 2008).

\subsection{Spitzer Multi-band Imaging Photometer for Spitzer (MIPS) Images}

The MIPS (Rieke et al. 2004) maps were created as part of the final products from the Spitzer Legacy Taurus I and II surveys (Padgett et al. 2007). The data were obtained in fast scan mode in three bands, 24, 70, and $160 \mu \mathrm{m}$, over an area of $44 \mathrm{deg}^{2}$. The observations were performed in three epochs between 2005 and 2007, with integration times of $30 \mathrm{~s}(24 \mu \mathrm{m})$ and $15 \mathrm{~s}(70$ and $160 \mu \mathrm{m})$. The maps were created using the basic calibrated data and coadded using the Spitzer software package Mosaicking and Point Source Extractor (Makovoz \& Marleau 2005). Despite the fact that the data were taken with interleaved scan legs to provide optimal coverage at 70 and $160 \mu \mathrm{m}$, some small gaps remained, in particular, at $160 \mu \mathrm{m}$. To mitigate this effect, the $160 \mu \mathrm{m}$ final mosaic was created using $32 \mathrm{arcsec}$ pixels, instead of the native $16 \mathrm{arcsec} / \mathrm{pixel}$ scale. This pixel scale matches quite well with the $\sim 40$ arcsec beam at $160 \mu \mathrm{m}$ wavelength. The 24 and $70 \mu \mathrm{m}$ maps adopted the standand 2.5 and 4 arcsec/pixel scale, respectively, to properly sample their respective 6 and 18 arcsec beams. The maps were used successfully for photometric purposes to identify new sources in the Taurus Molecular Cloud (Rebull et al. 2010).

\section{OUTFLOWS}

We identified 55 outflows around the Spitzer YSOs in the $44 \mathrm{deg}^{2}$ area of Taurus. In total, 31 of the detected outflows were previously unknown. In the following subsections, we describe the searching procedure of outflows, the morphology, physical properties, and the comparison between our findings and the known ones.

\subsection{The Search Procedures for Outflows}

Instead of a blind search, we focused on seeking outflows around YSOs. The search procedure was performed with an Interactive Data Language pipeline. We plotted spectra, position velocity diagrams (hereafter $\mathrm{P}-\mathrm{V}$ diagrams), and integrated intensity maps to identify the outflows around the 355 YSOs which Spitzer identified in Taurus. Detailed steps of the search are as follow.

1. We plotted ${ }^{12} \mathrm{CO}$ contours (hereafter contour map) overlaid on a ${ }^{13} \mathrm{CO}$ grayscale image around a YSO. According to the scale and velocity range of previously detected outflows in Taurus, we chose two sizes $\left(10^{\prime}\right.$ and $\left.20^{\prime}\right)$ and three sets of velocity intervals $(-1$ to $3.5 \mathrm{~km} \mathrm{~s}^{-1},-1$ to $4.5 \mathrm{~km} \mathrm{~s}^{-1}$, and -1 to $5.5 \mathrm{~km} \mathrm{~s}^{-1}$ for blue; $7.5-13 \mathrm{~km} \mathrm{~s}^{-1}, 8.5-13 \mathrm{~km} \mathrm{~s}^{-1}$, and $9.5-13 \mathrm{~km} \mathrm{~s}^{-1}$ for red) to plot the contour maps. We plotted the maps with three sets of velocity intervals and two scales automatically around the 355 YSOs. In total, 2130 maps were obtained. We inspected these maps to identify outflow candidates according to the morphology of the blue and red lobes. In the end, 74 candidates were selected.

2. We plotted ${ }^{12} \mathrm{CO} \mathrm{P}-\mathrm{V}$ diagrams along four directions (at position angles ${ }^{10}$ of $0^{\circ}, 45^{\circ}, 90^{\circ}$, and $135^{\circ}$ ) on three scales $\left(20^{\prime}, 40^{\prime}\right.$, and $\left.60^{\prime}\right)$ around the 74 candidates. The high-velocity range and size of outflow candidates were

\footnotetext{
${ }^{10}$ The position angle is defined as the angle measured from the north clockwise to the direction along which we plotted the P-V diagram.
} 
determined roughly by checking the P-V diagrams. When the velocity bulge appears in the direction away from the central velocity, we marked it as the start of the high-velocity wing. Along the above direction, the maximum velocity corresponding to the outermost contour is the end of this high-velocity wing. We further confirmed the high-velocity range of each candidate individually through visual inspection. The position range of the entire high-velocity bulge along the position axis was considered to be roughly the size of the outflow. If more than one central velocity is found in the $\mathrm{P}-\mathrm{V}$ diagram, then it likely has multiple velocity components (Wu et al. 2005), and thus will be excluded from the list of outflow candidates. Therefore, 19 candidates with multiple velocity components were eliminated and the remaining 55 outflow candidates were considered to be possible outflows.

3. Using the rough sizes and velocity ranges obtained in step 2 , we plotted contour maps for the remaining 55 outflows. P-V diagrams were plotted through the midpoint of the blue and red peaks (bipolar outflow) or through the peak of the lobe in the case of monopolar outflow, at position angles spaced by $15^{\circ}$. We chose the angle with the most prominent bulge along the velocity axis to determine the velocity interval of an outflow. Then, we plotted the contour map again with this velocity interval.

4. Finally, we plotted the average spectra of the blue and red lobes. According to the morphology of the P-V diagrams and contour maps, we divided the outflows into five classes. The higher the ranking, the more likely it is that we have identified an outflow. We define a typical $\mathrm{P}-\mathrm{V}$ diagram (TPV) and a representative contour map (RCM) as follows. If there is obvious high-velocity gas which can be seen by the protuberance along the velocity axis on the $\mathrm{P}-\mathrm{V}$ diagram and the high-velocity range is not less than $1 \mathrm{~km} \mathrm{~s}^{-1}$, then we regard the $\mathrm{P}-\mathrm{V}$ diagram as a TPV. If the outermost contour of the lobe is closed and we can see a clear and unbroken lobe on the contour map, then we regard the contour map as an RCM. Table 1 shows our criteria for outflow classification with an " $x$ ", meaning that it satisfies a certain condition. Having both ${ }^{12} \mathrm{CO}$ TPV and ${ }^{12} \mathrm{CO} \mathrm{RCM}$ is required for a high ranking (Class $\mathrm{A}^{+}$), but having ${ }^{13} \mathrm{CO}$ TPV or ${ }^{13} \mathrm{CO} \mathrm{RCM}$ gives a lower ranking (Class $\mathrm{A}^{-}$and Class $\mathrm{B}^{-}$) because ${ }^{13} \mathrm{CO}$ is generally optically thin in outflows and we usually found lobes of outflows with ${ }^{12} \mathrm{CO}$ not ${ }^{13} \mathrm{CO}$. Having only ${ }^{12} \mathrm{CO} \mathrm{RCM}$ provides the lowest ranking (Class $\mathrm{C}^{+}$) because the high-velocity gas in the $\mathrm{P}-\mathrm{V}$ diagram is not obvious. The primary condition to identify an outflow is having high-velocity gas that can be seen from the protuberance along the velocity axis on the $\mathrm{P}$ $\mathrm{V}$ diagram.

\subsection{The Results of Our Outflow Search}

Following the steps in Section 3.1, we found 55 outflows in the $44 \mathrm{deg}^{2}$ area of the Taurus molecular cloud. All of the outflows that we detected are listed in Table 2. Each outflow is referred as a "Taurus Molecular Outflow" (TMO). We present the locations, polarities, and scales of the outflows
Table 1

Criteria for Outflow Classification and Outflow Distribution

\begin{tabular}{lccccrr}
\hline \hline Class & ${ }^{12} \mathrm{CO}$ & ${ }^{12} \mathrm{CO}$ & ${ }^{13} \mathrm{CO}$ & ${ }^{13} \mathrm{CO}$ & $\begin{array}{r}\text { Outflow } \\
\text { Numbers }\end{array}$ & Percentage \\
& TPV & RCM & TPV & RCM & \\
\hline $\mathrm{A}^{+}$ & $\times$ & $\times$ & $\ldots$ & $\ldots$ & 24 & $43.6 \%$ \\
$\mathrm{~A}^{-}$ & $\times$ & $\times$ & $\times$ & $\times$ & 18 & $32.7 \%$ \\
$\mathrm{~B}^{+}$ & $\times$ & $\ldots$ & $\ldots$ & $\ldots$ & 4 & $7.3 \%$ \\
$\mathrm{~B}^{-}$ & $\times$ & $\ldots$ & $\times$ & $\ldots$ & 1 & $1.8 \%$ \\
$\mathrm{C}^{+}$ & $\ldots$ & $\times$ & $\ldots$ & $\ldots$ & 8 & $14.5 \%$ \\
\hline
\end{tabular}

overlaid on the Spitzer MIPS image in Figure 1. There are 31 new outlfows among all those detected. We have thus increased the total number of known outflows by a factor of 1.3 .

Table 1 lists the numbers and percentages in the five classes of outflows. We can see that Class $\mathrm{A}^{+}$and class $\mathrm{A}^{-}$account for $76.3 \%$ of all of the detected outflows. These two types can be considered as the "most probable" outflows in our study. Table 3 lists the numbers of previously known and newly detected outflows in different classes. We found more new outflows of Class $\mathrm{A}^{+}$and class $\mathrm{A}^{-}$, which account for $64.5 \%$ of all the newly detected outflows. That is, most of the new outflows we found are likely true outflows.

Table 4 lists the outflow numbers and percentages according to the types of their driving sources. Class I accounts for $36.4 \%$, which is the largest proportion of all the YSOs driving outflow. The outflows driven by the Class I YSOs are closer to the YSOs and have more collimated bipolar morphology. Compared with Class I, Class III YSOs drive a small proportion $(12.7 \%)$ of outflows, which tend to be farther from the YSOs. This indicates that the outflows from Class III YSOs are more evolved than those from Class I YSOs. We also found three outflows (TMO_13, TMO_40, and TMO 46) without YSOs, indicating that they are possibly Class 0 objects. Among the three outflows, TMO_13 and $\mathrm{TMO}_{-} 40$ are newly discovered in our study while TMO_46 has $\bar{b}$ been reported in Narayanan et al. (2012).

\subsection{Morphology of Outflows}

We found 25 bipolar, 22 monopolar redshifted, and 6 monopolar blueshifted outflows. Bipolar and redshifted outflows account for the vast majority of outflows in the Taurus molecular cloud. This is consistent with the results of Narayanan et al. (2012). Figures 2-56 show the ${ }^{12} \mathrm{CO}$ integrated intensity map, the ${ }^{12} \mathrm{CO} \mathrm{P}-\mathrm{V}$ diagram, and the average spectrum for each outflow. For Class $\mathrm{A}^{-}$and $\mathrm{B}^{-}$ outflows, we also plotted the ${ }^{13} \mathrm{CO}$ integrated intensity maps and ${ }^{13} \mathrm{CO} \mathrm{P}-\mathrm{V}$ diagrams.

\subsection{Comparison with Previously Discovered Outflows}

Using FCRAO large-scale survey data (Narayanan et al. 2008) and the latest YSO catalog from Rebull et al. (2010), we were able to identify previously known outflows, obtain more complete morphology, and find additional new outflows. The YSO catalog is also convenient for identifying the driving sources of the outflows. Through comparison with previous works, we confirmed more driving sources of outflows. 
Table 2

Outflows in Taurus

\begin{tabular}{|c|c|c|c|c|c|c|c|c|}
\hline $\begin{array}{l}\text { Outflow } \\
\text { Name }\end{array}$ & $\begin{array}{c}\text { R.A. } \\
(\mathrm{J} 2000)\end{array}$ & $\begin{array}{c}\text { decl. } \\
(\mathrm{J} 2000)\end{array}$ & $\begin{array}{l}\text { Common } \\
\text { Name }\end{array}$ & $\begin{array}{l}\text { YSO } \\
\text { Type }^{\text {a }}\end{array}$ & $\begin{array}{c}\text { Outflow } \\
\text { Class }\end{array}$ & Po. $^{\text {b }}$ & $\begin{array}{c}\text { New } \\
\text { Detection }^{\mathrm{c}}\end{array}$ & Reference \\
\hline TMO_01 & 041159.7 & 294236 & $\ldots$ & III & $\mathrm{A}^{+}$ & MR & $\mathrm{N}$ & 1 \\
\hline TMO_02 & 041412.2 & 280837 & IRAS $04113+2758$ (L1495) & I & $\mathrm{A}^{+}$ & $\mathrm{Bi}$ & $\mathrm{N}$ & $1,2,3$ \\
\hline TMO_03 & 041414.5 & 282758 & $\ldots$ & II & $\mathrm{A}^{+}$ & $\mathrm{Bi}$ & $\mathrm{Y}$ & 4 \\
\hline TMO_04 & 041832.0 & 283115 & $\cdots$ & Flat & $\mathrm{A}^{+}$ & $\mathrm{Bi}$ & $\mathrm{Y}$ & 4 \\
\hline TMO_05 & 041941.4 & 271607 & IRAS $04166+2706$ & I & $\mathrm{A}^{+}$ & MB & $\mathrm{N}$ & $1,2,5,6,7$ \\
\hline TMO_06 & 041958.4 & 270957 & IRAS 04169+2702 & I & $\mathrm{A}^{+}$ & $\mathrm{Bi}$ & $\mathrm{N}$ & $1,2,3,7$ \\
\hline TMO_07 & 042107.9 & 270220 & IRAS $04181+2655$ & I & $\mathrm{A}^{+}$ & $\mathrm{Bi}$ & $\mathrm{N}$ & $2,3,6,7$ \\
\hline TMO_08 & 042215.6 & 265706 & FS Tau B & I & $\mathrm{A}^{+}$ & $\mathrm{Bi}$ & $\mathrm{N}$ & 1,2 \\
\hline TMO_09 & 042325.9 & 250354 & $\ldots$ & II & $\mathrm{A}^{+}$ & MR & $\mathrm{Y}$ & 4 \\
\hline TMO_10 & 042420.9 & 263051 & $\ldots$ & II & $\mathrm{A}^{+}$ & $\mathrm{Bi}$ & $\mathrm{Y}$ & 4 \\
\hline TMO_11 & 042445.0 & 270144 & $\ldots$ & III & $\mathrm{A}^{+}$ & MR & $\mathrm{Y}$ & 4 \\
\hline TMO_12 & 042930.0 & 243955 & Haro 6-10 & I & $\mathrm{A}^{+}$ & MR & $\mathrm{N}$ & $1,6,8$ \\
\hline TMO_13 & 043110.4 & 254129 & $\ldots$ & $\cdots$ & $\mathrm{A}^{+}$ & MR & $\mathrm{Y}$ & 4 \\
\hline TMO_14 & 043158.4 & 254329 & $\ldots$ & III & $\mathrm{A}^{+}$ & $\mathrm{Bi}$ & $\mathrm{Y}$ & 4 \\
\hline TMO_15 & 043214.6 & 223742 & $\ldots$ & Flat & $\mathrm{A}^{+}$ & $\mathrm{Bi}$ & $\mathrm{Y}$ & 4 \\
\hline TMO_16 & 043231.7 & 242002 & L1529 & II & $\mathrm{A}^{+}$ & $\mathrm{Bi}$ & $\mathrm{N}$ & $1,6,9,10$ \\
\hline TMO_17 & 043232.0 & 225726 & IRAS 04295+2251 (L1536) & I & $\mathrm{A}^{+}$ & MR & $\mathrm{N}$ & 3,7 \\
\hline TMO_18 & 043243.0 & 255231 & $\cdots$ & II & $\mathrm{A}^{+}$ & $\mathrm{Bi}$ & $\mathrm{Y}$ & 4 \\
\hline TMO_19 & 043415.2 & 225030 & $\cdots$ & II & $\mathrm{A}^{+}$ & MR & $\mathrm{Y}$ & 4 \\
\hline TMO_20 & 043724.8 & 270919 & $\cdots$ & III & $\mathrm{A}^{+}$ & MR & $\mathrm{Y}$ & 4 \\
\hline TMO_21 & 043953.9 & 260309 & L1527 & I & $\mathrm{A}^{+}$ & $\mathrm{Bi}$ & $\mathrm{N}$ & $1,3,6,7,11,12,13$ \\
\hline TMO_22 & 044108.2 & 255607 & IRAS $04381+2540$ (TMC-1) & Flat & $\mathrm{A}^{+}$ & MB & $\mathrm{N}$ & $1,6,7,14$ \\
\hline TMO_23 & 044112.6 & 254635 & IRAS $04381+2540$ (TMC-1) & I & $\mathrm{A}^{+}$ & MR & $\mathrm{N}$ & $1,6,7,14$ \\
\hline TMO_24 & 044207.7 & 252311 & IRAS $04390+2517(\mathrm{LkH} \alpha 332)$ & II & $\mathrm{A}^{+}$ & MR & $\mathrm{N}$ & 3 \\
\hline TMO_25 & 041858.1 & 281223 & IRAS 04158+2805 (L1495) & Flat & $\mathrm{A}^{-}$ & MB & $\mathrm{Y}$ & 4,7 \\
\hline TMO_26 & 042318.2 & 264115 & $\cdots$ & II & $\mathrm{A}^{-}$ & MR & $\mathrm{Y}$ & 4 \\
\hline TMO_27 & 042656.2 & 244335 & IRAS $04239+2436($ HH 300) & I & $\mathrm{A}^{-}$ & MR & $\mathrm{N}$ & $1,3,6,15$ \\
\hline TMO_28 & 042702.6 & 260530 & IRAS 04240+2559 (DG Tau) & I & $\mathrm{A}^{-}$ & $\mathrm{Bi}$ & $\mathrm{N}$ & 1,16 \\
\hline TMO_29 & 042702.8 & 254222 & $\cdots$ & II & $\mathrm{A}^{-}$ & $\mathrm{Bi}$ & $\mathrm{Y}$ & 4 \\
\hline TMO_30 & 042757.3 & 261918 & IRAS $04248+2612$ & Flat & $\mathrm{A}^{-}$ & MR & $\mathrm{N}$ & 1,3 \\
\hline TMO_31 & 042810.4 & 243553 & $\cdots$ & Flat & $\mathrm{A}^{-}$ & MR & $\mathrm{Y}$ & 4 \\
\hline TMO_32 & 043051.7 & 244147 & IRAS 04278+2435 (ZZ Tau IRS) & Flat & $\mathrm{A}^{-}$ & MR & $\mathrm{N}$ & $1,6,17$ \\
\hline TMO_33 & 043215.4 & 242859 & IRAS 04292+2422 (Haro 6-13) & Flat & $\mathrm{A}^{-}$ & $\mathrm{Bi}$ & $\mathrm{N}$ & 1,3 \\
\hline TMO_34 & 043307.8 & 261606 & $\cdots$ & III & $\mathrm{A}^{-}$ & MB & $\mathrm{Y}$ & 4 \\
\hline TMO_35 & 043310.0 & 243343 & $\cdots$ & III & $\mathrm{A}^{-}$ & MR & $\mathrm{Y}$ & 4 \\
\hline TMO_36 & 043316.5 & 225320 & IRAS $04302+2247$ & I & $\mathrm{A}^{-}$ & $\mathrm{Bi}$ & $\mathrm{N}$ & $1,3,7$ \\
\hline TMO_37 & 043334.0 & 242117 & $\cdots$ & II & $\mathrm{A}^{-}$ & MR & $\mathrm{Y}$ & 4 \\
\hline TMO_38 & 043336.7 & 260949 & $\cdots$ & II & $\mathrm{A}^{-}$ & MB & $\mathrm{Y}$ & 4 \\
\hline TMO_39 & 043557.6 & 225357 & IRAS $04328+2248$ (HP Tau) & II & $\mathrm{A}^{-}$ & $\mathrm{Bi}$ & $\mathrm{N}$ & 3 \\
\hline TMO_40 & 043911.2 & 252710 & HH706 & $\cdots$ & $\mathrm{A}^{-}$ & MR & $\mathrm{N}$ & 1 \\
\hline TMO_41 & 043913.8 & 255320 & IRAS $04361+2547$ (TMR-1) & I & $\mathrm{A}^{-}$ & $\mathrm{Bi}$ & $\mathrm{N}$ & $1,3,6,7,18$ \\
\hline TMO_42 & 044802.3 & 253359 & Tau A 8 & III & $\mathrm{A}^{-}$ & $\mathrm{Bi}$ & $\mathrm{Y}$ & 4 \\
\hline TMO_43 & 041851.4 & 282026 & HH156 & I & $\mathrm{B}^{+}$ & MB & $\mathrm{Y}$ & 2 \\
\hline TMO_44 & 042021.4 & 281349 & $\cdots$ & Flat & $\mathrm{B}^{+}$ & $\mathrm{Bi}$ & $\mathrm{Y}$ & 4 \\
\hline TMO_45 & 042653.3 & 255858 & $\cdots$ & I & $\mathrm{B}^{+}$ & $\mathrm{Bi}$ & $\mathrm{Y}$ & 4 \\
\hline TMO_46 & 043956.1 & 262802 & $\cdots$ & $\cdots$ & $\mathrm{B}^{+}$ & $\mathrm{Bi}$ & $\mathrm{Y}$ & 4 \\
\hline TMO_47 & 043535.3 & 240819 & IRAS $04325+2402$ (L1535) & I & $\mathrm{B}^{-}$ & $\mathrm{Bi}$ & $\mathrm{N}$ & $1,3,6,17,19,20$ \\
\hline TMO_48 & 041535.6 & 284741 & $\cdots$ & I & $\mathrm{C}^{+}$ & $\mathrm{Bi}$ & $\mathrm{Y}$ & 4 \\
\hline TMO_49 & 041733.7 & 282046 & $\cdots$ & II & $\mathrm{C}^{+}$ & MR & $\mathrm{Y}$ & 4 \\
\hline TMO_50 & 041810.5 & 284447 & $\cdots$ & I & $\mathrm{C}^{+}$ & MR & $\mathrm{Y}$ & 4 \\
\hline TMO_51 & 041831.1 & 281629 & $\cdots$ & II & $\mathrm{C}^{+}$ & MR & $\mathrm{Y}$ & 4 \\
\hline TMO_52 & 041831.2 & 282617 & $\cdots$ & I & $\mathrm{C}^{+}$ & MR & $\mathrm{Y}$ & 4 \\
\hline TMO_53 & 041841.3 & 282725 & $\cdots$ & Flat & $\mathrm{C}^{+}$ & MR & $\mathrm{Y}$ & 4 \\
\hline TMO_54 & 042154.5 & 265231 & $\cdots$ & II & $\mathrm{C}^{+}$ & $\mathrm{Bi}$ & $\mathrm{Y}$ & 4 \\
\hline TMO_55 & 042904.9 & 264907 & IRAS $04260+2642$ & I & $\mathrm{C}^{+}$ & MR & $\mathrm{N}$ & 4 \\
\hline
\end{tabular}

Notes.

a The YSO classification from Rebull et al. (2010). "Flat" represents a flat-spectrum YSO, which is intermediate between Class I and II.

b The polarity of the outflow. "Bi" represents bipolar outflow, and "MB" and "MR" indicate blue and red monopolar outflow, respectively.

${ }^{\mathrm{c}}$ This column represents whether the outflow is detected for the first time in our study. $\mathrm{Y}=$ new, $\mathrm{N}=$ has reported in previous work.

References. (1) Narayanan et al. (2012), (2) Davis et al. (2010), (3) Moriarty-Schieven et al. (1992), (4) this paper, (5) Tafalla et al. (2004), (6) Wu et al. (2004), (7) Bontemps et al. (1996), (8) Stojimirović et al. (2007), (9) Lichten (1982), (10) Goldsmith et al. (1984), (11) Tamura et al. (1996), (12) Hogerheijde et al. (1998), (13) Zhou et al. (1996); (14) Chandler et al. (1996), (15) Arce \& Goodman (2001), (16) Mitchell et al. (1994), (17) Heyer et al. (1987), (18) Terebey et al. (1990), (19) Myers et al. (1988), (20) Wu et al. (1992). 


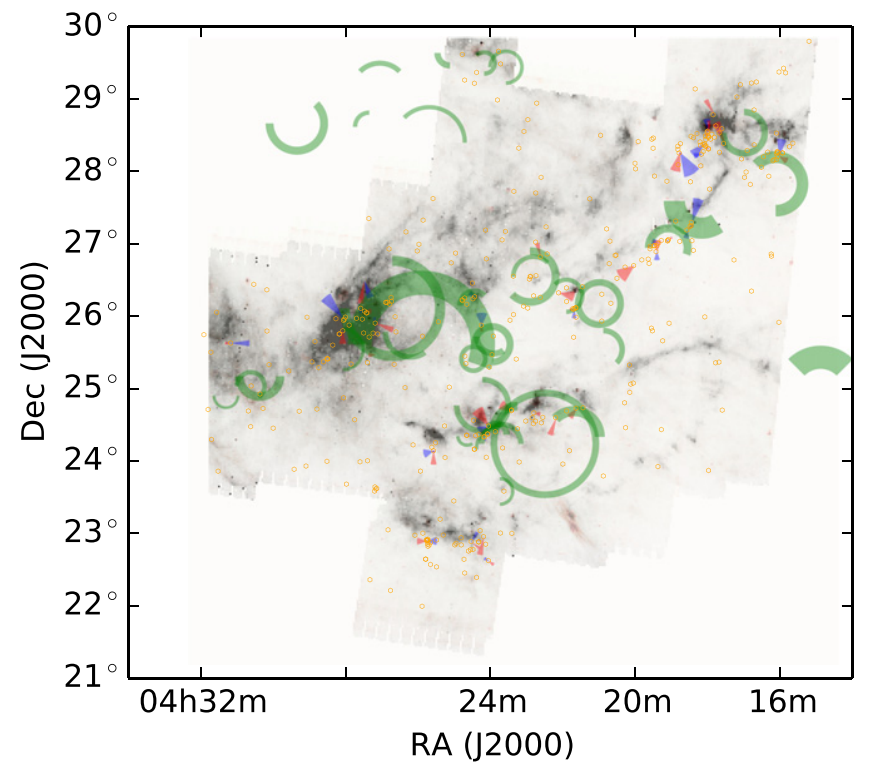

Figure 1. Outflows and bubbles overlaid on the Spitzer $160 \mu \mathrm{m}$ (gray), $70 \mu \mathrm{m}$ (green), and $24 \mu \mathrm{m}$ (red) image from the Spitzer Legacy Taurus I and II surveys. The blue and red sectors represent the blue and red lobes of outflows in Table 2, respectively. The radius of the sectors shows the scale of the outflow, while the direction of the sectors shows the projected direction of the outflow. The green rings and arcs represent the expanding and broken bubbles, respectively. The thickness and radius of the arcs and rings are the actual thickness and radius of the bubble structures. The open orange circles show the locations of YSOs, which were listed in Tables 6 and 7 of Rebull et al. (2010).

Table 3

The Numbers of Outflows in Different Classes

\begin{tabular}{lcc}
\hline \hline Type & $\begin{array}{c}\text { Previously } \\
\text { Known }\end{array}$ & $\begin{array}{c}\text { Newly } \\
\text { Detected }\end{array}$ \\
\hline $\mathrm{A}^{+}$ & 13 & 11 \\
$\mathrm{~A}^{-}$ & 9 & 9 \\
$\mathrm{~B}^{+}$ & 0 & 4 \\
$\mathrm{~B}^{-}$ & 1 & 0 \\
$\mathrm{C}^{+}$ & 1 & 7 \\
\hline
\end{tabular}

Table 4

Number of Outflows Around Different Types of YSOs

\begin{tabular}{lrr}
\hline \hline YSO & $\begin{array}{r}\text { Number of } \\
\text { Outflows }\end{array}$ & Percentage \\
Type & 20 & $36.4 \%$ \\
\hline I & 10 & $18.2 \%$ \\
Flat & 15 & $27.3 \%$ \\
II & 7 & $12.7 \%$ \\
III & 3 & $5.4 \%$ \\
No YSO & 3 & \\
\hline
\end{tabular}

L1527 (TMO_21) is a typical outflow in Taurus (Zhou et al. 1996; Hogerheijde et al. 1998; Narayanan et al. 2012). The P-V diagram and contour map in our work are very similar to those in Hogerheijde et al. (1998) and Narayanan et al. (2012). TMO_08 (SST 042215.6+265706) and FS Tau B in Narayanan et al. (2012) are the same outflow with the same location. They have the same structure, which can be seen in our Figure 9 and
Figure 15 in Narayanan et al. (2012). In addition, TMO 30 (SST042757.3+261918), TMO_32 (SST043051.7+244147), TMO_33 (SST 043215.4+242859), and TMO_41 (SST043913.8+255320) also have the same morphology as IRAS 04248+2612, ZZ Tau IRS, IRAS 04292+2422, and IRAS $04361+2547$ in Narayanan et al. (2012), respectively. These confirm the general consistency between the two works in terms of strong and extended outflows.

For TMO_02 (SST041412.2+280837), we obtained a good bipolar structure, shown in the upper left panel of Figure 2, while Narayanan et al. (2008) considered this outflow (IRAS $04113+2758$ ) to be only redshifted. Davis et al. (2010) did not identify the driving source of this outflow (named the W-CO-flow1), but we determined that the YSO SST $041412.2+280837$ is driving the outflow. MoriartySchieven et al. (1992) only presented the central spectrum of IRAS $04390+2517$ and IRAS $04328+2248$, while we illustrated the two outflows (TMO_24 and TMO_39) more clearly through contour maps and $\overline{\mathrm{P}}-\mathrm{V}$ diagrams.

The morphology of TMO_07 (SST042107.9+270220) shown in Figure 8 is similar to that of J04210795+2702204 in Davis et al. (2010). This outflow was also reported by Moriarty-Schieven et al. (1992), Bontemps et al. (1996), and $\mathrm{Wu}$ et al. (2004). However, Narayanan et al. (2012) did not find it using the same FCRAO survey data. Mitchell et al. (1994) reported that IRAS $04240+2559$ was a monopolar redshifted outflow with the ${ }^{12} \mathrm{CO}(3-2)$ data. However, at this location, we found the well-defined bipolar outflow as shown in Figure 29.

As for L1529, Lichten (1982) presented high-velocity ${ }^{12} \mathrm{CO}$ wings observed by antenna No. 2 of the Caltech $10.4 \mathrm{~m}$ array, but Goldsmith et al. (1984) did not find any high-velocity gas in observations at FCRAO. We identified a bipolar outflow named TMO_16 (SST043231.7+242002) and demonstrated the result of Lichten (1982) with the FCRAO data. At the position of IRAS 04295+2251, Moriarty-Schieven et al. (1992) showed line wings while Bontemps et al. (1996) found no outflow. We found a red monopolar outflow as shown in Figure 18.

We have found 31 new outflows which are labeled "Y" in the eighth column of Table 2. Two of these new outflows were not identified as outflows in the literature. Bontemps et al. (1996) considered IRAS 04158+2805 (L1495) but did not find any sign of outflow activity in the ${ }^{12} \mathrm{CO}(2-1)$ transition at the location of TMO_025 (SST041858.1 +281223). Davis et al. (2010) had some doubt about the CO flow of CoKU Tau-1 when analyzing the ${ }^{12} \mathrm{CO}(3-2)$ emission, but we found TMO_043 (SST041851.4+282026) at this site. The rest of the new outflows have not been reported in the literature and are identified as outflows for the first time. All of the new outflows are of small angular extent, less than $10^{\prime}$. They may have been missed in previous searches because of their small sizes.

\subsection{Physical Parameters of Outflows}

To study the effects of outflows on their environment, we calculated their masses, momenta, kinetic energy, and energy deposition rates. The total column density of the outflowing gas 

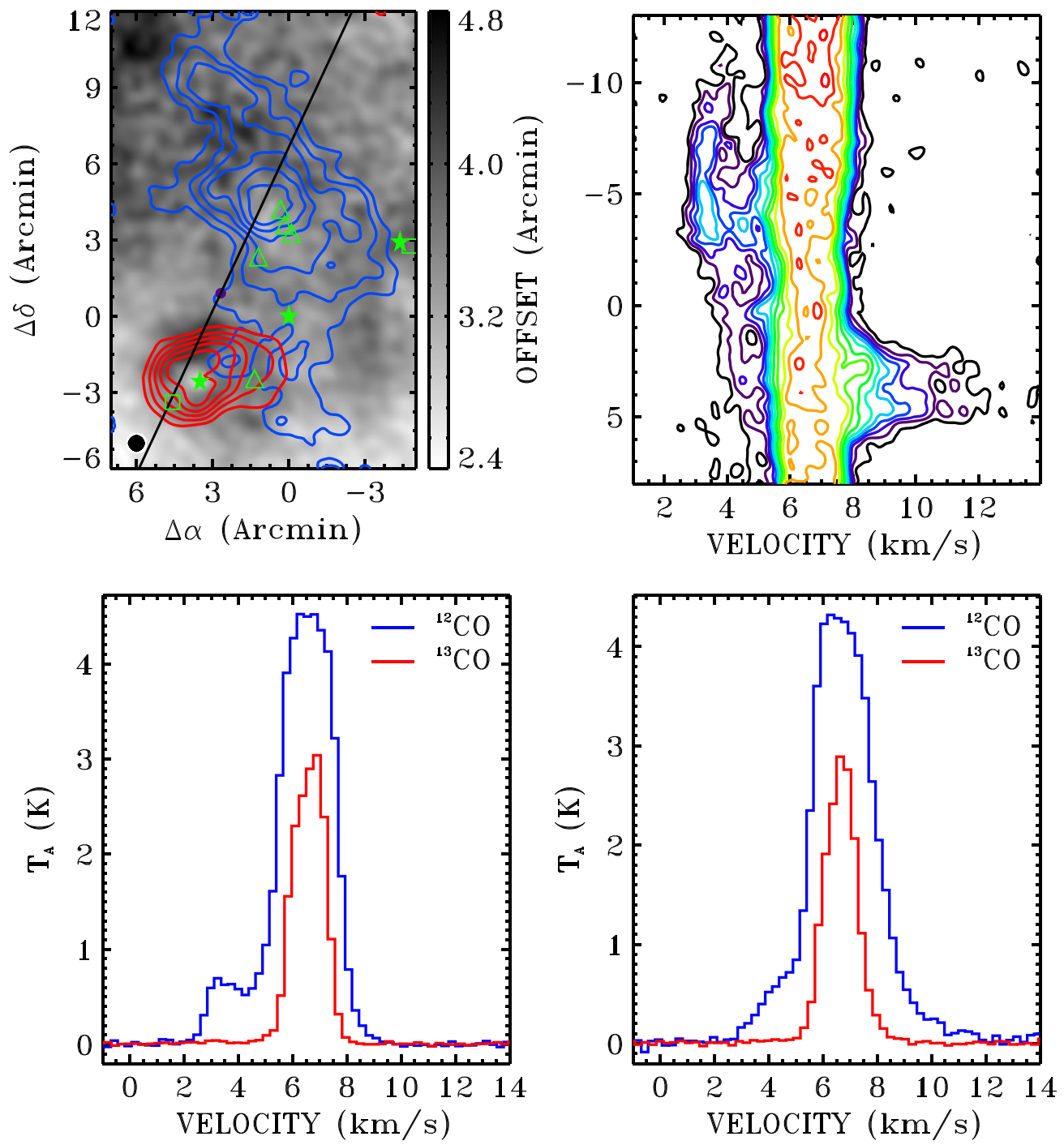

Figure 2. TMO 02 (SST 041412.2+280837). Upper left panel: ${ }^{12} \mathrm{CO}$ integrated intensity map overlaid on the ${ }^{13} \mathrm{CO}$ grayscale image, integrated over $2-5 \mathrm{~km} \mathrm{~s}{ }^{-1}$ for the blue lobe and $8-12.5 \mathrm{~km} \mathrm{~s}^{-1}$ for the red lobe with ${ }^{12} \mathrm{CO}$, and integrated over $5.5-7.5 \mathrm{~km} \mathrm{~s}^{-1}$ for the ${ }^{13} \mathrm{CO}$ grayscale image. The blue and red contour levels are $40 \%$, $50 \%, \ldots, 90 \%$ and $30 \%, 45 \%, \ldots, 90 \%$ of their peak value, respectively. In this panel and all of the subsequent upper left panels, including those of bubbles, the green filled stars, if present, show the location of Class I YSOs in Taurus, the green open squares show the location of flat-spectrum YSOs, the green open triangles show the location of Class II YSOs, and the green filled squares show the location of Class III YSOs. All of the above YSOs were listed in Tables 6 and 7 of Rebull et al. (2010). The black solid line represents a cut for the P-V diagram shown in the upper right panel, and the purple filled circle indicates the origin on the $Y$-axis of the upper right panel. The filled circle in the lower left corner shows the beam. Upper right panel: $\mathrm{P}-\mathrm{V}$ diagram of ${ }^{12} \mathrm{CO}$ through the slice shown in the upper left panel at a position angle of $25^{\circ}$. Contour levels are $0.4-1.6 \mathrm{~K}$ by $0.3 \mathrm{~K}, 2-5 \mathrm{~K}$ by $0.5 \mathrm{~K}$. Lower left panel: average spectra of ${ }^{12} \mathrm{CO}$ emission (blue lines) and ${ }^{13} \mathrm{CO}$ emission (red lines) toward the blueshifted lobe shown in the upper left panel. Lower right panel: average spectra of ${ }^{12} \mathrm{CO}$ emission (blue lines) and ${ }^{13} \mathrm{CO}$ emission (red lines) toward the redshifted lobe shown in the upper left panel.

is

$$
N_{\text {tot }}\left({ }^{12} \mathrm{CO}\right)=\frac{3 k^{2} T_{\mathrm{ex}}}{4 \pi^{3} \mu_{\mathrm{d}}^{2} h \nu^{2} \exp \left(-h \nu / k T_{\mathrm{ex}}\right)} \int T_{\mathrm{s}} d \nu,
$$

where $k=1.38 \times 10^{-16} \mathrm{erg} \mathrm{K}^{-1}, h=6.626 \times 10^{-27} \mathrm{erg} \mathrm{s}$, $\mu_{\mathrm{d}}=0.112 \times 10^{-18} \mathrm{esu}, \nu=115.2712 \times 10^{9} \mathrm{~Hz}$, and $T_{\mathrm{s}}$ is the observed source antenna temperature with proper correction for antenna efficiency. We assumed an excitation temperature of $25 \mathrm{~K}$. The excitation temperature assumed in the literature (Tamura et al. 1996; Zhou et al. 1996; Ohashi et al. 1997a, 1997b; Davis et al. 2010; Narayanan et al. 2012) ranges from 11 to $50 \mathrm{~K}$. The lowest temperature will decrease the mass estimate by a factor of 3 and the highest temperature will increase the mass estimate by a factor of 2.2. The detailed derivations regarding the physical parameters of the outflows are given in the appendix.

Arce \& Goodman (2001) described three major issues that can cause uncertainties in the calculation of an outflow's parameters, namely, the inclination, opacity, and blending. Our prescriptions are as follow. (1) We defined the inclination angle of the outflow as the angle between the long axis of the outflow and the line of sight. Since the outflows with small inclination angle (especially when the outflow is perpendicular to the plane of the sky) are hard to detect, our outflow search is biased toward those with large inclination angle. If the 

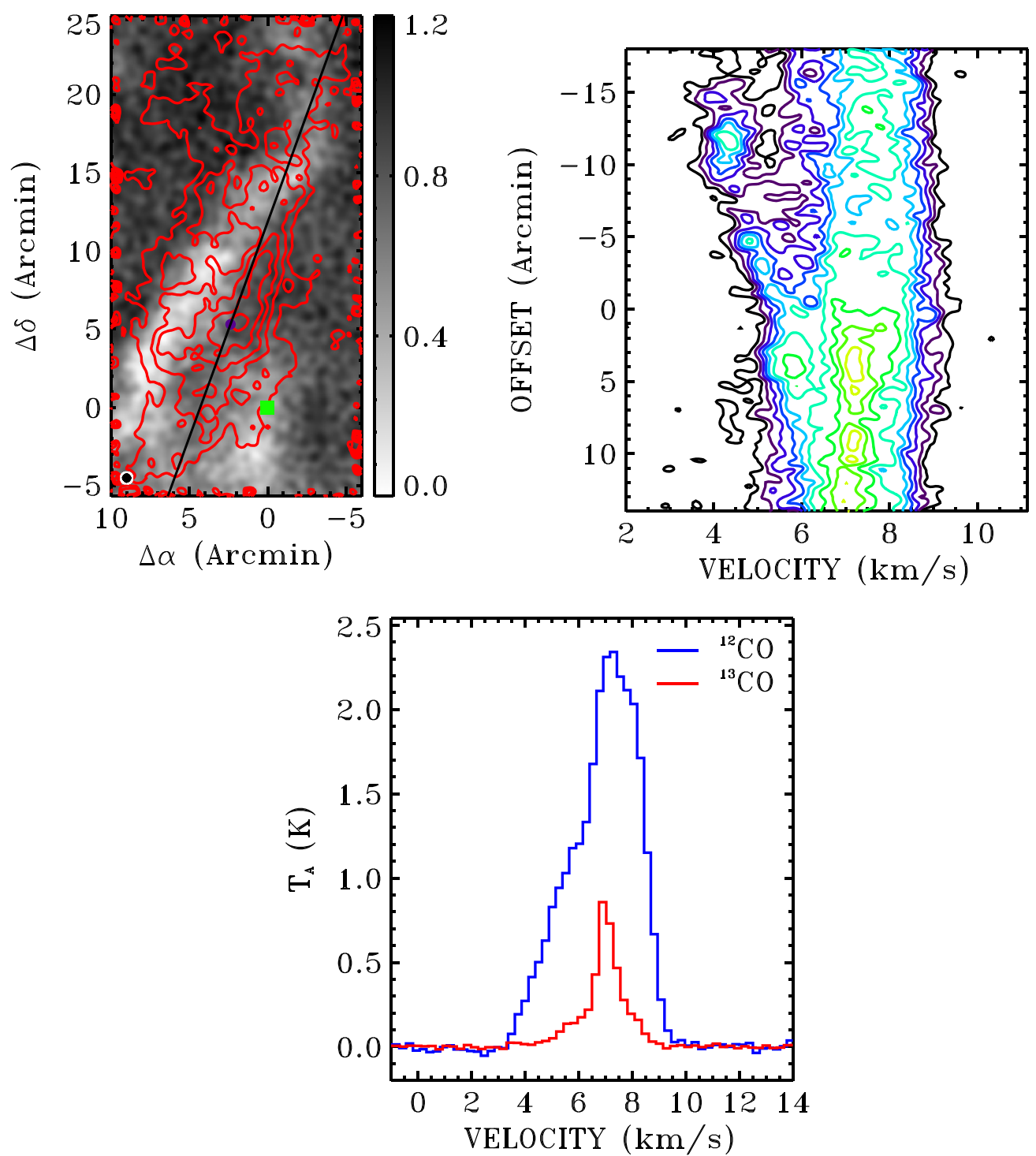

Figure 3. TMO_01 (SST 041159.7+294236). Upper left and upper right panels: same as the upper two panels of Figure 2 except for the following. In the upper left panel, the integrated intervals for the red lobe and grayscale images are $8.75-10 \mathrm{~km} \mathrm{~s}^{-1}$ and $6.5-7.5 \mathrm{~km} \mathrm{~s}^{-1}$, respectively. In the upper right panel, the position angle is $20^{\circ}$ and the contour levels are $0.4-1.6 \mathrm{~K}$ by $0.3 \mathrm{~K}, 2-4 \mathrm{~K}$ by $0.5 \mathrm{~K}$. Lower panel: same as the lower right panel of Figure 2.

inclination angle $\theta$ is randomly distributed, then the average value is given by

$$
\langle\theta\rangle=\int_{0}^{\pi / 2} \theta \sin \theta d \theta .
$$

From the above formula, we obtained the average inclination angle of $57: 3$, which differs from the typically used median value of $45^{\circ}$. Then, the velocity and dynamic age, $t_{\mathrm{dyn}}$, should be scaled up by a factor of 1.9 and 0.64 , respectively. (2) Using the ${ }^{12} \mathrm{CO}$ and ${ }^{13} \mathrm{CO}$ data, we can correct for the opacity in the ${ }^{12} \mathrm{CO}$ line when the ${ }^{12} \mathrm{CO}$ emission of an outflow is optically thick. The algorithm for the opacity correction is described in the Appendix. (3) We probably missed some low-velocity outflowing gas which blended into the ambient gas when we conservatively determined the emission from outflows only. Previous studies (Margulis \& Lada 1985; Arce et al. 2010; Narayanan et al. 2012) showed that neglecting this gas results in the underestimate of the outflow mass by nearly a factor of two.

Table 5 gives the length, mass, momentum, kinetic energy, dynamical timescale, and luminosity of the outflows in Taurus.

The distributions of length, mass, energy, and dynamical timescale of outflows are shown in Figure 57. The extents of the outflows are in the range $0.1-1.11 \mathrm{pc} .79 \%$ of outflows are smaller than $0.6 \mathrm{pc}$. The masses of $54 \%$ of outflows are between $0.01 M_{\odot}$ and $0.1 M_{\odot}$. The outflows with masses lower than $0.01 M_{\odot}$ and higher than $0.1 M_{\odot}$ account for $17 \%$ and $29 \%$ of the total, respectively. The energy of $48 \%$ of outflows is in the range $10^{42}-10^{43} \mathrm{erg}$. The outflows with energy lower than $10^{42} \mathrm{erg}$ and higher than $10^{43} \mathrm{erg}$ account for $31 \%$ and $21 \%$, respectively. The dynamical timescales of outflows are between $0.3 \times 10^{5}$ years and $6.1 \times 10^{5}$ years. $85 \%$ of outflows have dynamical timescales shorter than $2.5 \times 10^{5}$ years. 

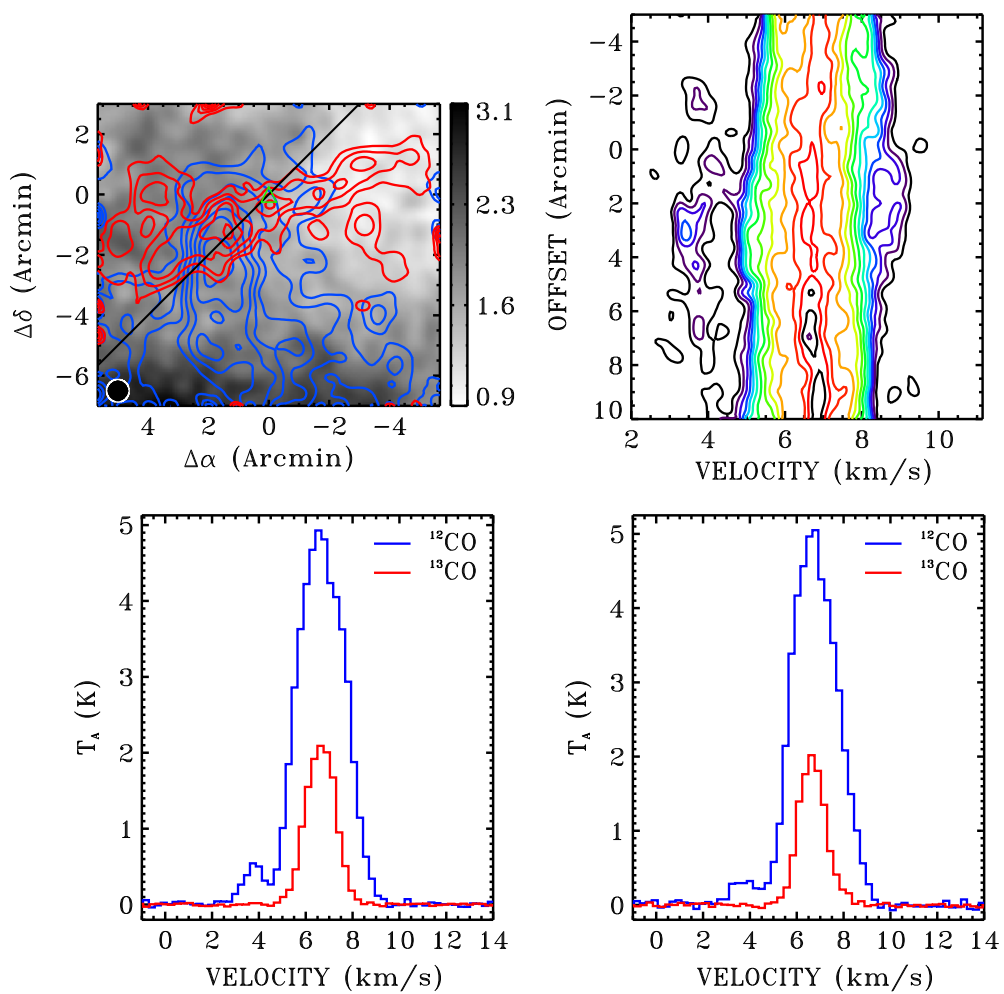

Figure 4. TMO_03 (SST 041414.5+282758). Same as Figure 2 except for the following. In the upper left panel, the integrated intervals for the red lobe and grayscale image are $8.5-9.5 \mathrm{~km} \mathrm{~s}^{-1}$ and $6-7 \mathrm{~km} \mathrm{~s}^{-1}$, respectively. The blue and red contour levels are $30 \%, 40 \%, \ldots, 90 \%$ and $50 \%, 60 \%, \ldots, 90 \%$ of their peak value, respectively. In the upper right panel, the position angle is $45^{\circ}$ and the contour levels are $0.4-1.6 \mathrm{~K}$ by $0.3 \mathrm{~K}, 2-6 \mathrm{~K}$ by $0.5 \mathrm{~K}$.
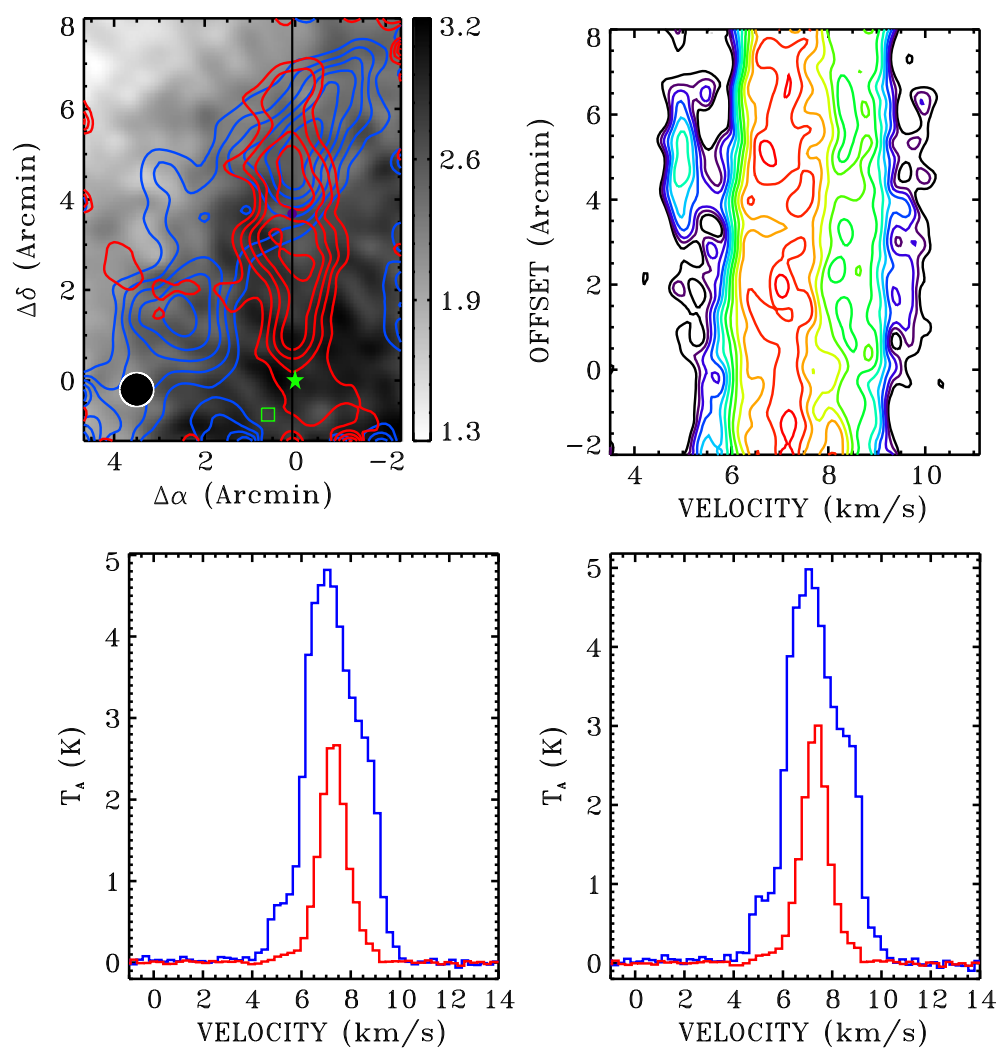

Figure 5. TMO_04 (SST 041832.0+283115). Same as Figure 2 except for the following. In the upper left panel, the integrated intervals for blue lobe, red lobe, and grayscale images are $4.4-5.3 \mathrm{~km} \mathrm{~s}^{-1}, 9.5-10.3 \mathrm{~km} \mathrm{~s}^{-1}$, and $6.5-8 \mathrm{~km} \mathrm{~s}^{-1}$, respectively. The red contour levels are $35 \%, 50 \%, \ldots, \% 90$ of the peak value. In the upper right panel, the position angle is $0^{\circ}$ and the contour levels are $0 .-2 \mathrm{~K}$ by $0.3 \mathrm{~K}, 2.5-5.5 \mathrm{~K}$ by $0.5 \mathrm{~K}$. 

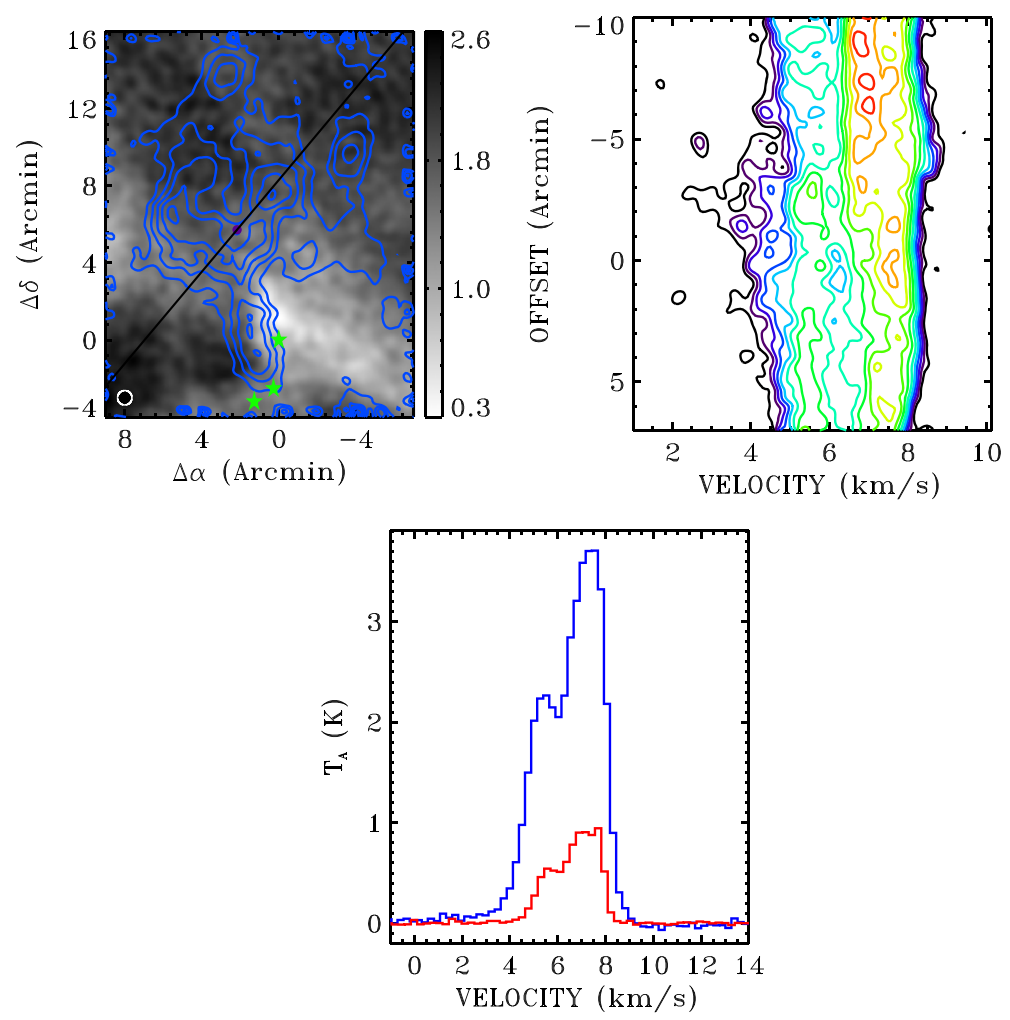

Figure 6. TMO_05 (SST 041941.4+271607). Upper left and upper right. Same as upper two panels of Figure 2 except for the following. In the upper left panel, the integrated intervals for the blue lobe and grayscale images are $2-4.3 \mathrm{~km} \mathrm{~s}^{-1}$ and $6-7 \mathrm{~km} \mathrm{~s}^{-1}$, respectively. The blue levels are $30 \%, 45 \%, \ldots, 90 \%$ of the peak value. In the upper right panel, the position angle is $40^{\circ}$ and the contour levels are $0.3-0.9 \mathrm{~K}$ by $0.15 \mathrm{~K}, 1.1-1.9 \mathrm{~K}$ by $0.2 \mathrm{~K}$. Lower panel. Same as the lower left panel of Figure 2.
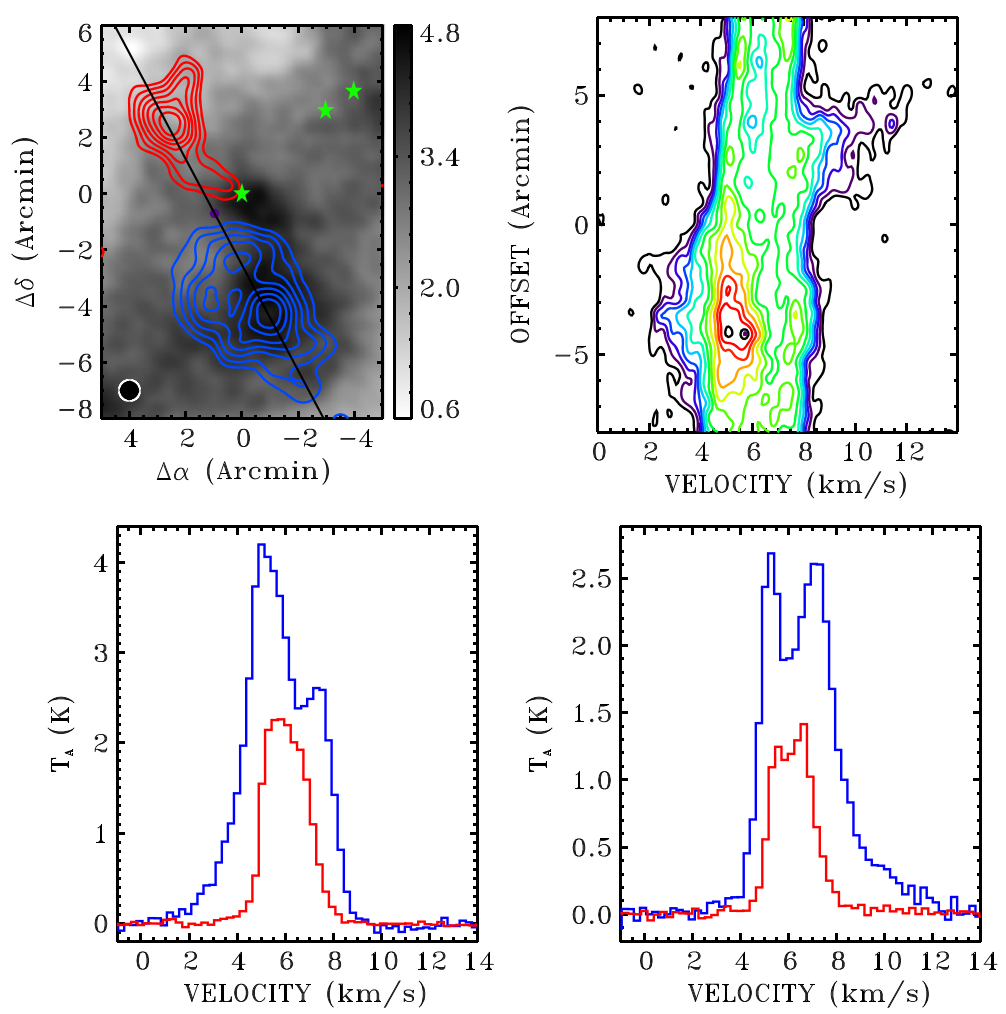

Figure 7. TMO_06 (SST 041958.4+270957). Same as Figure 2 except for the following. In the upper left panel, the integrated intervals for the blue lobe, red lobe, and grayscale images are $2-4 \mathrm{~km} \mathrm{~s}^{-1}, 9-12 \mathrm{~km} \mathrm{~s}^{-1}$, and $5-7 \mathrm{~km} \mathrm{~s}^{-1}$, respectively. The blue and red contour levels are both $30 \%, 40 \%, \ldots, 90 \%$ of their peak value. In the upper right panel, the position angle is $152^{\circ}$ and contour levels are $0.5-2 \mathrm{~K}$ by $0.3 \mathrm{~K}, 2.5-5.5 \mathrm{~K}$ by $0.5 \mathrm{~K}$. 

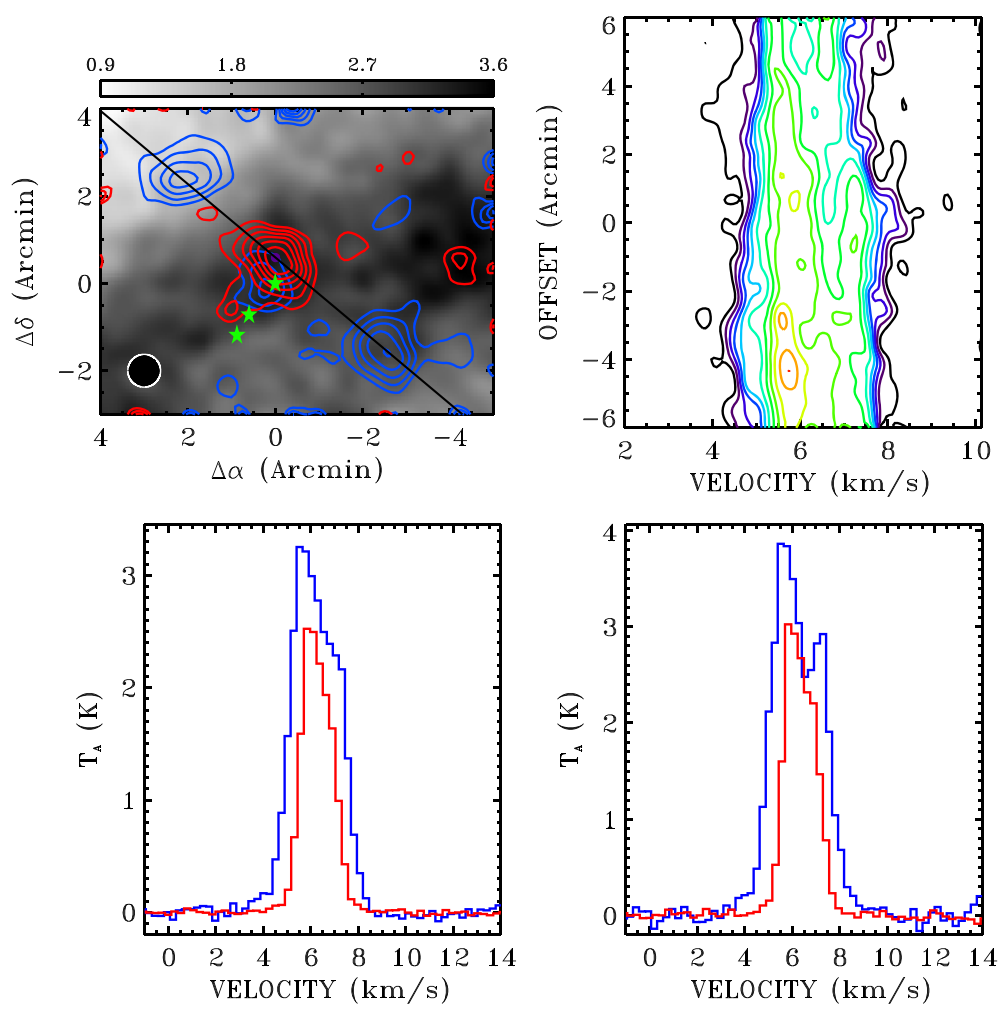

Figure 8. TMO 07 (SST 042107.9+270220). Same as Figure 2 except for the following. In the upper left panel, the integrated intervals for the blue lobe and red lobe are $3-4.5 \mathrm{~km} \mathrm{~s}^{-\Gamma}$ and $8-9 \mathrm{~km} \mathrm{~s}^{-1}$, respectively. The blue and red contour levels are $50 \%, 60 \%, \ldots, 90 \%$ and $40 \%, 50 \%, \ldots, 90 \%$ of their peak value, respectively. In the upper right panel, the position angle is $130^{\circ}$ and contour levels are $0.5-2 \mathrm{~K}$ by $0.3 \mathrm{~K}, 2.5-4.5 \mathrm{~K}$ by $0.5 \mathrm{~K}$.
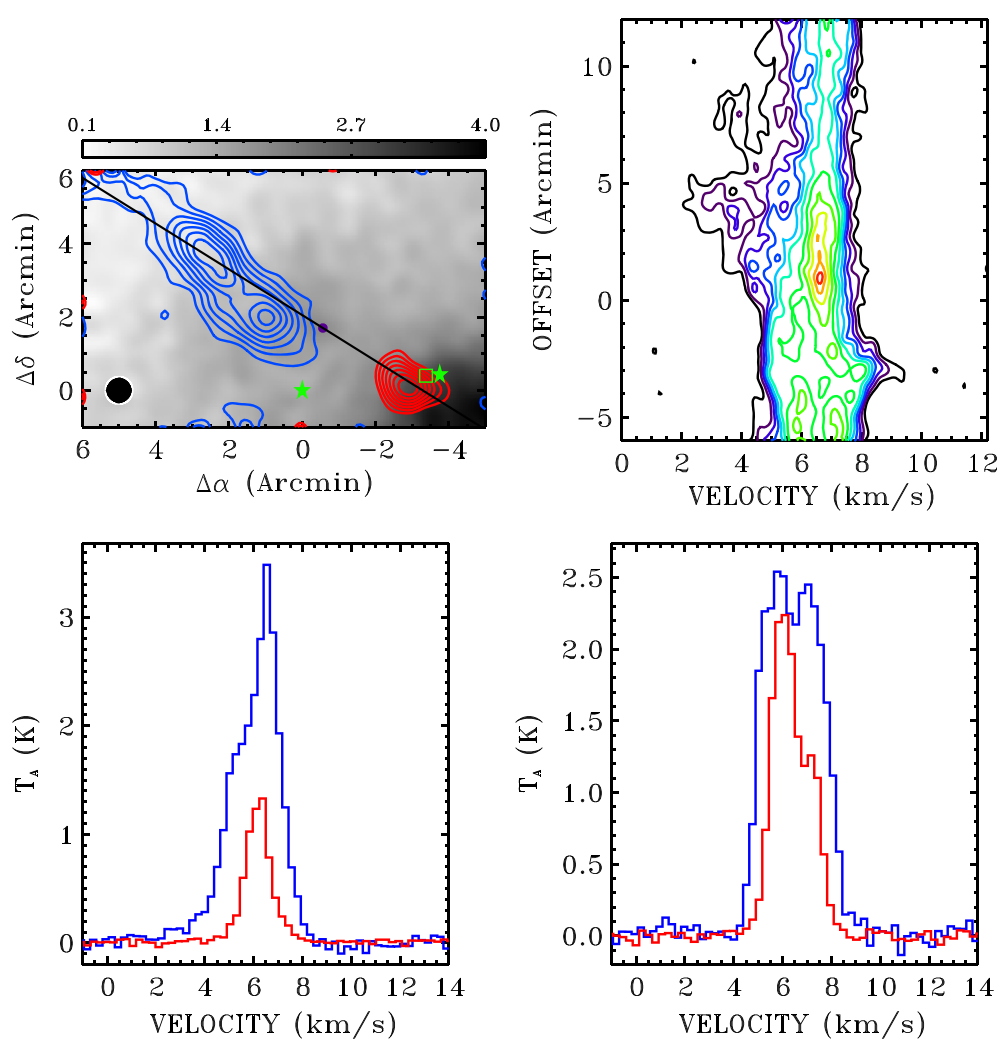

Figure 9. TMO 08 (SST 042215.6+265706). Same as Figure 2 except for the following. In the upper left panel, the integrated intervals for the blue lobe, red lobe, and grayscale images are $2-4.5 \mathrm{~km} \mathrm{~s}^{-1}, 8.3-9.5 \mathrm{~km} \mathrm{~s}^{-1}$, and $5.5-7 \mathrm{~km} \mathrm{~s}^{-1}$, respectively. The blue and red contour levels are both $30 \%, 40 \%, \ldots, 90 \%$ of their peak value. In the upper right panel, the position angle is $122^{\circ}$ and the contour levels are $0.5-2 \mathrm{~K}$ by $0.3 \mathrm{~K}, 2.5-4 \mathrm{~K}$ by $0.5 \mathrm{~K}$. 

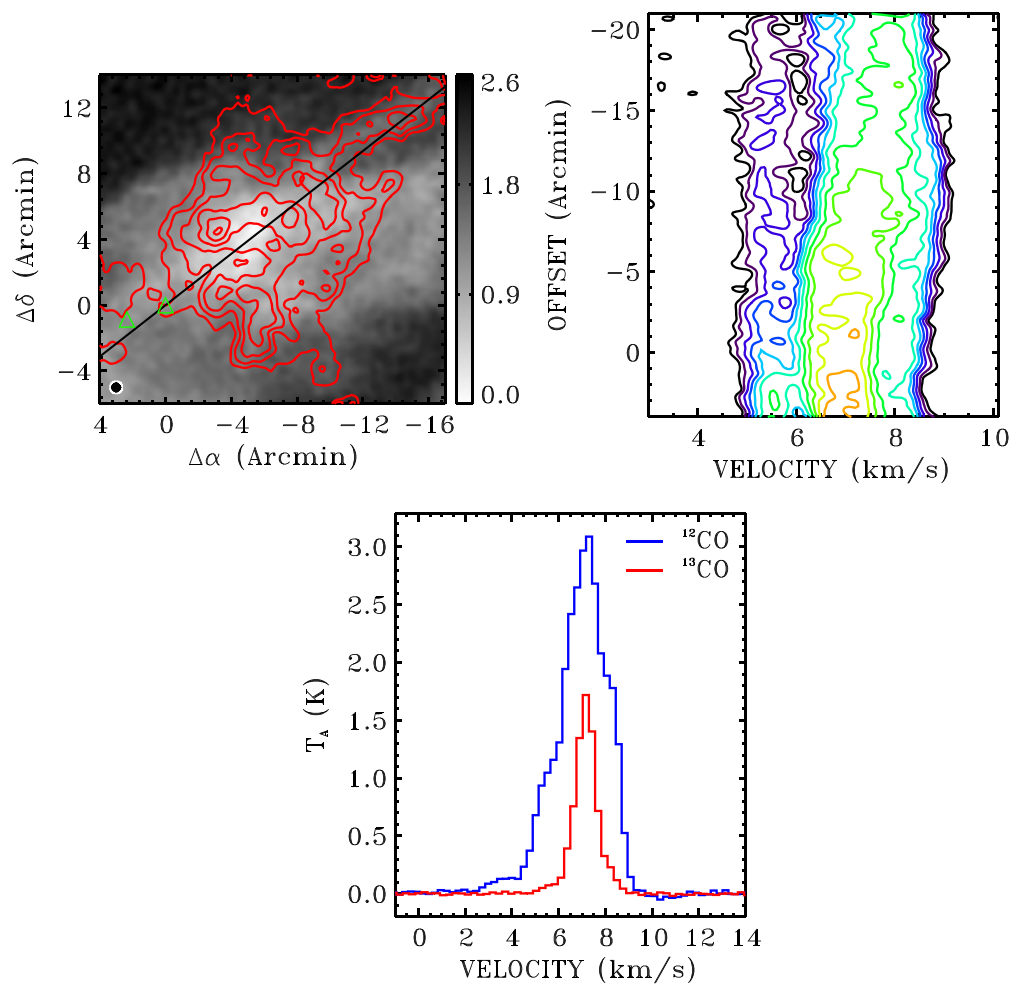

Figure 10. TMO_09 (SST 042325.9+250354). Same as Figure 3 except for the following. In the upper left panel, the integrated interval for the red lobe is $8.25-9.7 \mathrm{~km} \mathrm{~s}^{-1}$. The red contour levels are $50 \%, 60 \%, \ldots, 90 \%$ of the peak value. In the upper right panel, the position angle is $52^{\circ}$ and the contour levels are $0.4-1.6 \mathrm{~K}$ by $0.3 \mathrm{~K}, 2-4.5$ by $0.5 \mathrm{~K}$.
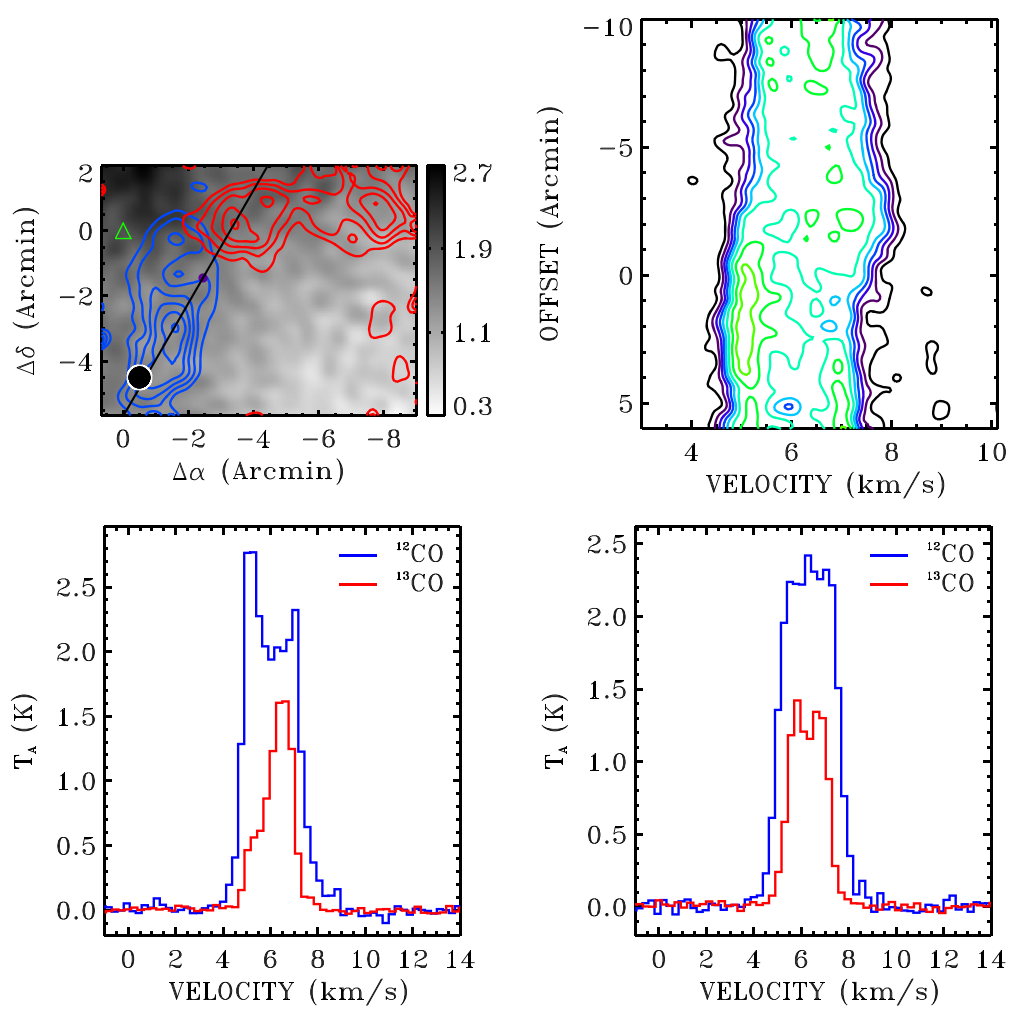

Figure 11. TMO_10 (SST 042420.9+263051). Same as Figure 2 except for the following. In the upper left panel, the integrated intervals for the blue lobe, red lobe, and grayscale images are $4-4.75 \mathrm{~km} \mathrm{~s}^{-1}, 7.5-10 \mathrm{~km} \mathrm{~s}^{-1}$, and $5.5-7.25 \mathrm{~km} \mathrm{~s}^{-1}$, respectively. The blue and red contour levels are $50 \%, 60 \%, \ldots, 90 \%$ and $60 \%, 70 \%, \ldots$, $90 \%$ of their peak value, respectively. In the upper right panel, the position angle is $30^{\circ}$ and the contour levels are $0.4-1.6 \mathrm{~K}$ by $0.3 \mathrm{~K}, 2-7 \mathrm{~K}$ by $0.5 \mathrm{~K}$. 

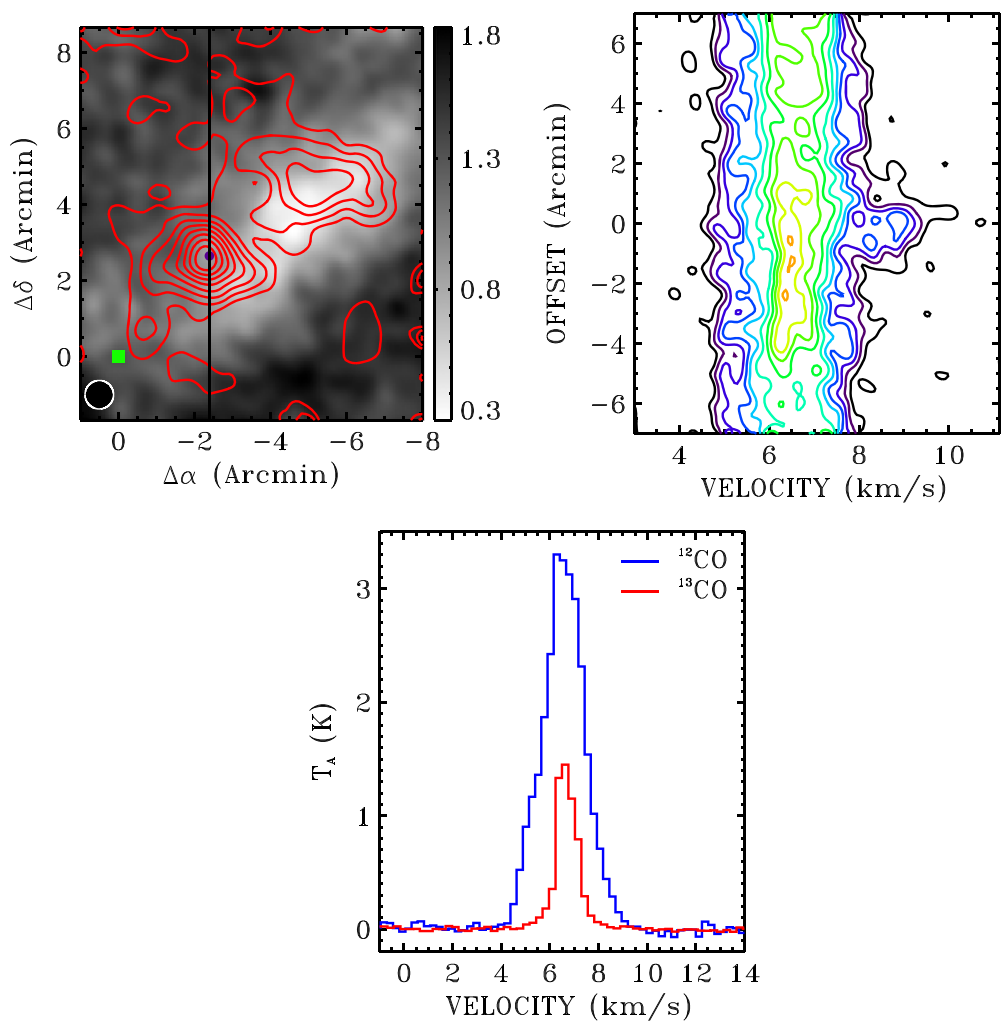

Figure 12. TMO_11 (SST 042445.0+270144). Same as Figure 3 except for the following. In the upper left panel, the integrated intervals for the red lobe and grayscale images are $8-10.5 \mathrm{~km} \mathrm{~s}^{-1}$ and $6-7 \mathrm{~km} \mathrm{~s}^{-1}$, respectively. The red contour levels are $20 \%, 30 \%, \ldots, 90 \%$ of the peak value. In the upper right panel, the position angle is $0^{\circ}$.
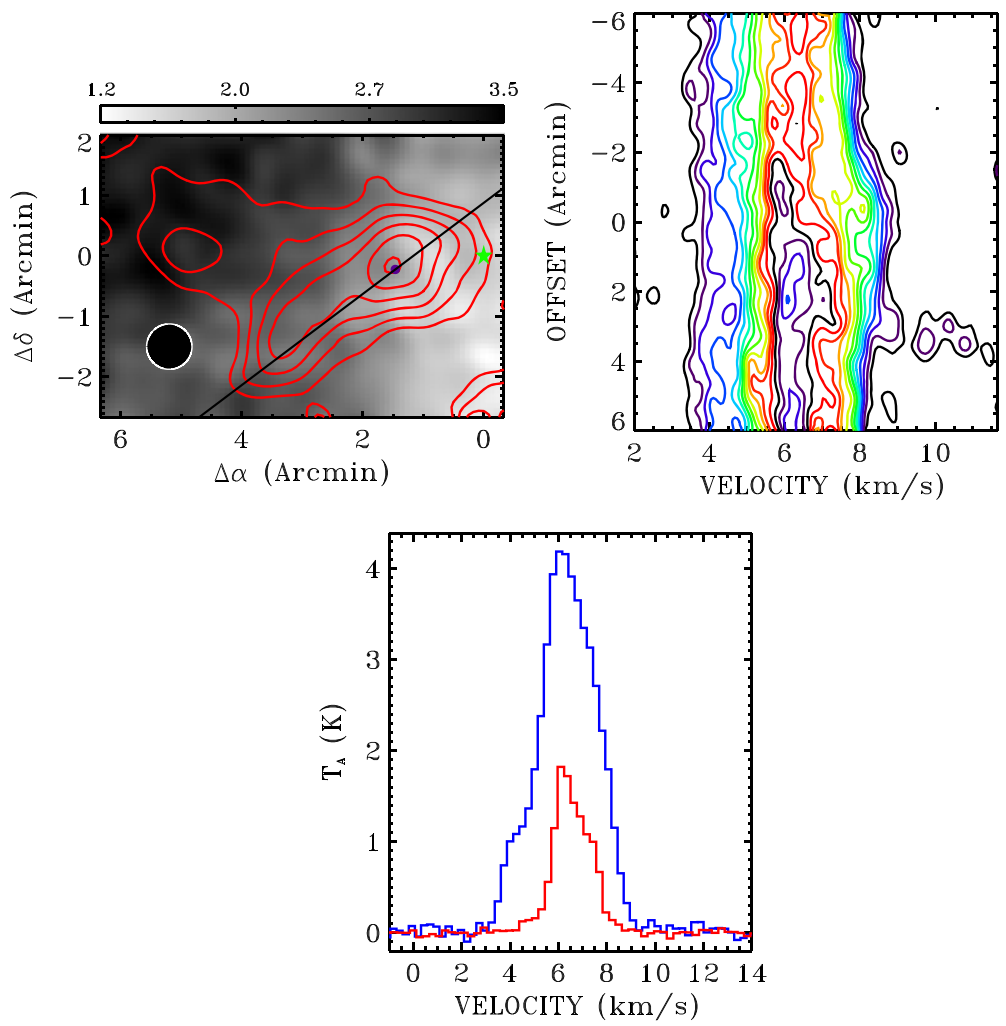

Figure 13. TMO 12 (SST 042930.0+243955). Same as Figure 3 except for the following. In the upper left panel, the integrated intervals for the red lobe and grayscale images are $8-9.2 \mathrm{~km} \mathrm{~s}^{-1}$ and $5.5-7.5 \mathrm{~km} \mathrm{~s}^{-1}$, respectively. The red contour levels are $50 \%, 60 \%, \ldots, 90 \%$ of the peak value. In the upper right panel, the position angle is $53^{\circ}$ and the contour levels are $0.4-4.8 \mathrm{~K}$ by $0.3 \mathrm{~K}$. 

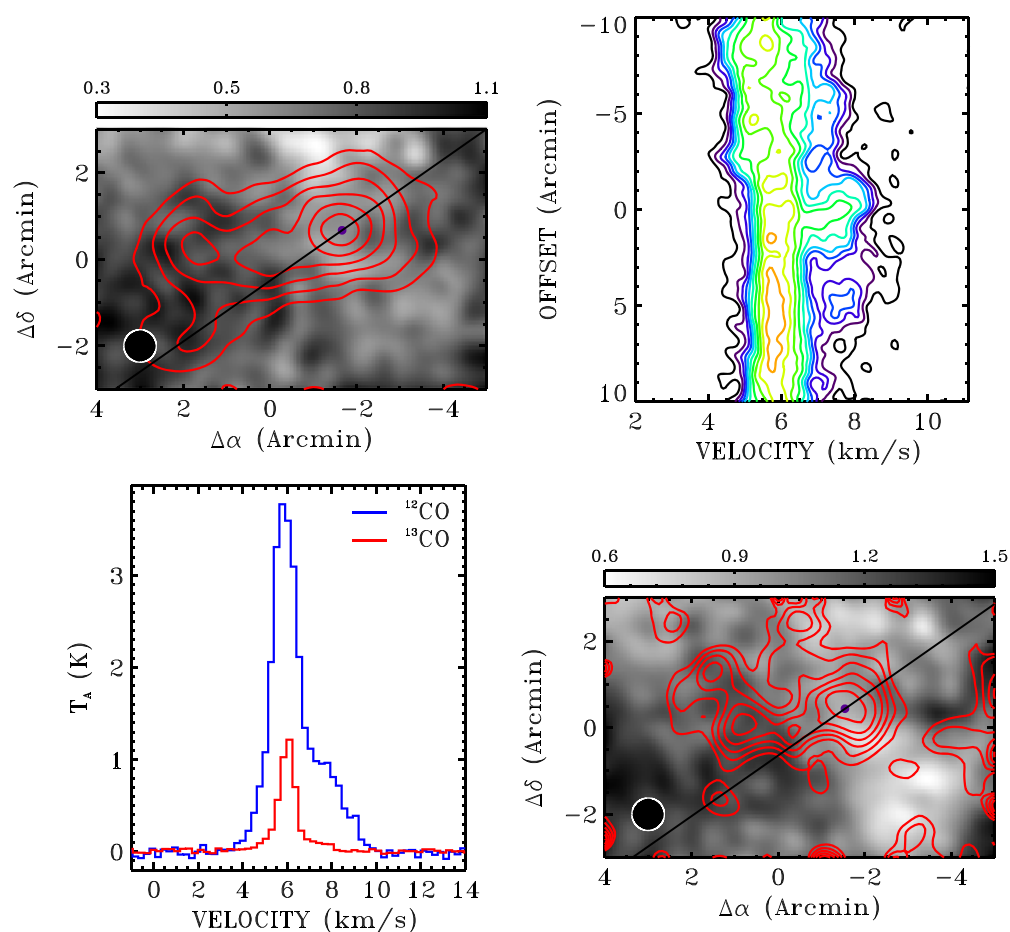

Figure 14. TMO 13. Upper left and upper right: same as the upper two panels of Figure 3 except for the following. In the upper left panel, the integrated intervals for the red lobe and grayscale images are $7-9.5 \mathrm{~km} \mathrm{~s}^{-1}$ and $5.5-6.5 \mathrm{~km} \mathrm{~s}^{-1}$, respectively. The red contour levels are $40 \%, 50 \%, \ldots, 90 \%$ of the peak value. In the upper right panel, the position angle is $55^{\circ}$ and the contour levels are $0.4-1.6 \mathrm{~K}$ by $0.3 \mathrm{~K}, 2-4.5 \mathrm{~K}$ by $0.5 \mathrm{~K}$. Lower left panel: same as the lower panel of Figure 3 . Lower right panel: ${ }^{13} \mathrm{CO}$ integrated intensity map overlaid on the ${ }^{13} \mathrm{CO}$ grayscale image, the integrated intervals for the red lobe, and the grayscale images and the red contour levels are the same with those in the upper left panel of this figure.
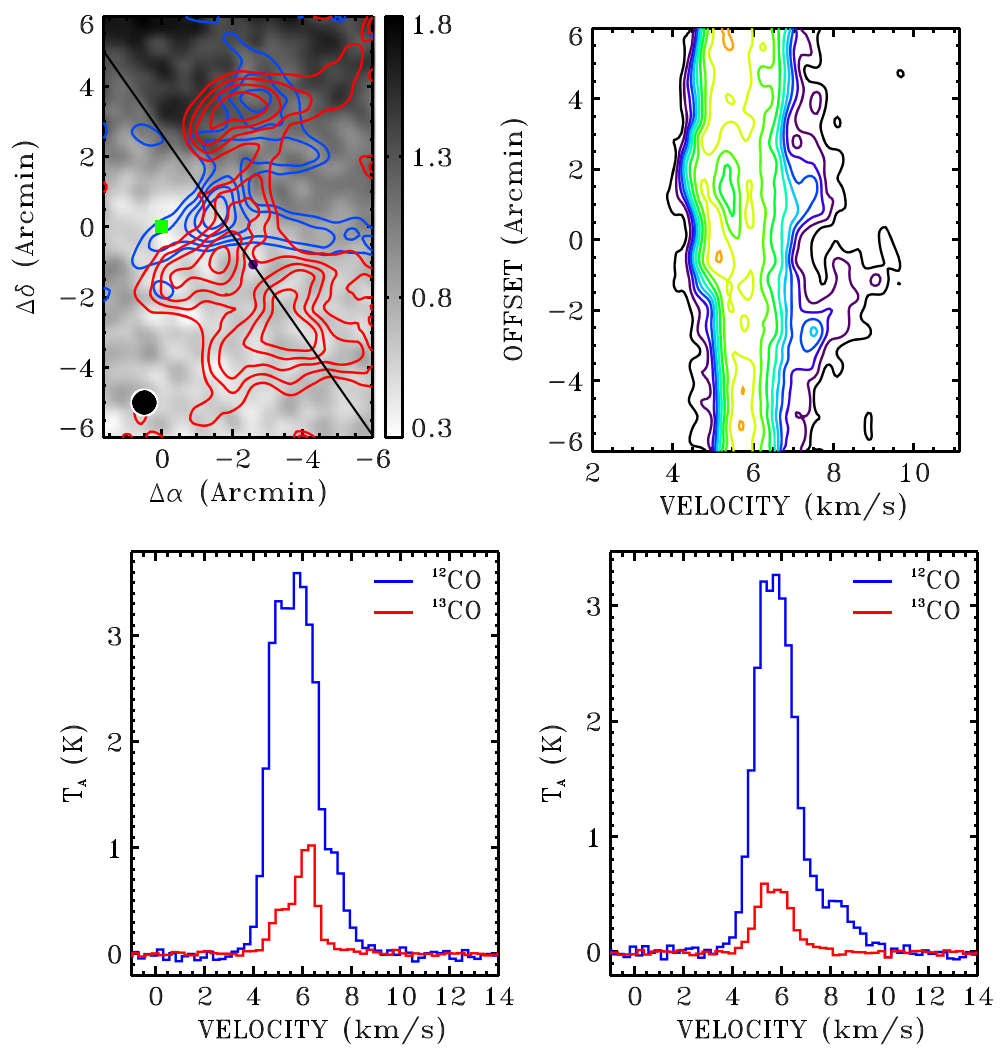

Figure 15. TMO_14 (SST 043158.4+254329). Same as Figure 2 except for the following. In the upper left panel, the integrated intervals for the blue lobe, red lobe, and grayscale images are $3-4.5 \mathrm{~km} \mathrm{~s}^{-1}, 7-10 \mathrm{~km} \mathrm{~s}^{-1}$, and $5-6.5 \mathrm{~km} \mathrm{~s}^{-1}$, respectively. The blue and red contour levels are both $50 \%, 60 \%, \ldots, 90 \%$ of their peak value. In the upper right panel, the position angle is $145^{\circ}$ and the contour levels are $0.4-1.6 \mathrm{~K}$ by $0.3 \mathrm{~K}, 2-4.5 \mathrm{~K}$ by $0.5 \mathrm{~K}$. 

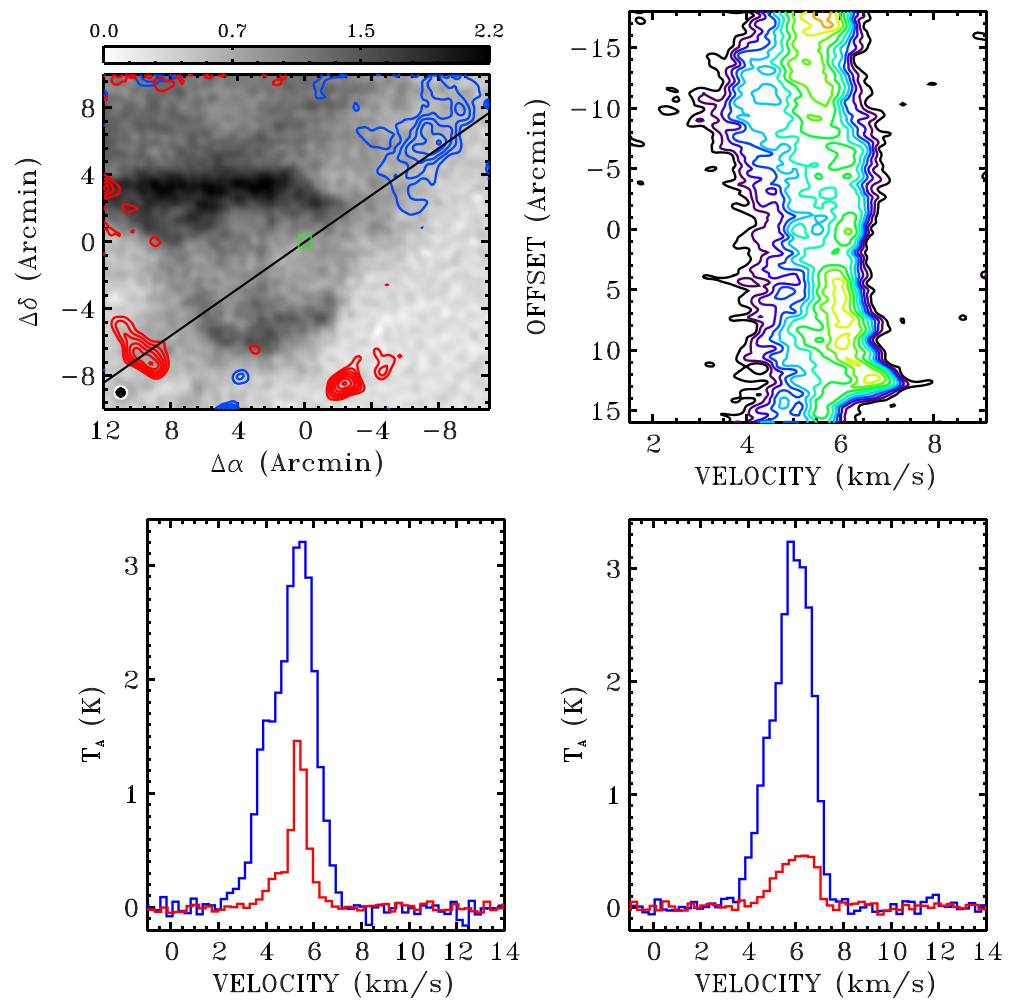

Figure 16. TMO 15 (SST 043214.6+223742). Same as Figure 2 except for the following. In the upper left panel, the integrated intervals for the blue lobe, red lobe, and gray-scale images are $2.8-4 \mathrm{~km} \mathrm{~s}^{-1}, 6.8-9 \mathrm{~km} \mathrm{~s}^{-1}$, and $5-6 \mathrm{~km} \mathrm{~s}^{-1}$, respectively. The blue and red contour levels are $60 \%, 70 \%, \ldots, 90 \%$ and $40 \%, 50 \%, \ldots, 90 \%$ of their peak value, respectively. In the upper right panel, the position angle is $55^{\circ}$ and the contour levels are $0.5-2 \mathrm{~K}$ by $0.3 \mathrm{~K}, 2.5-4 \mathrm{~K}$ by $0.5 \mathrm{~K}$.
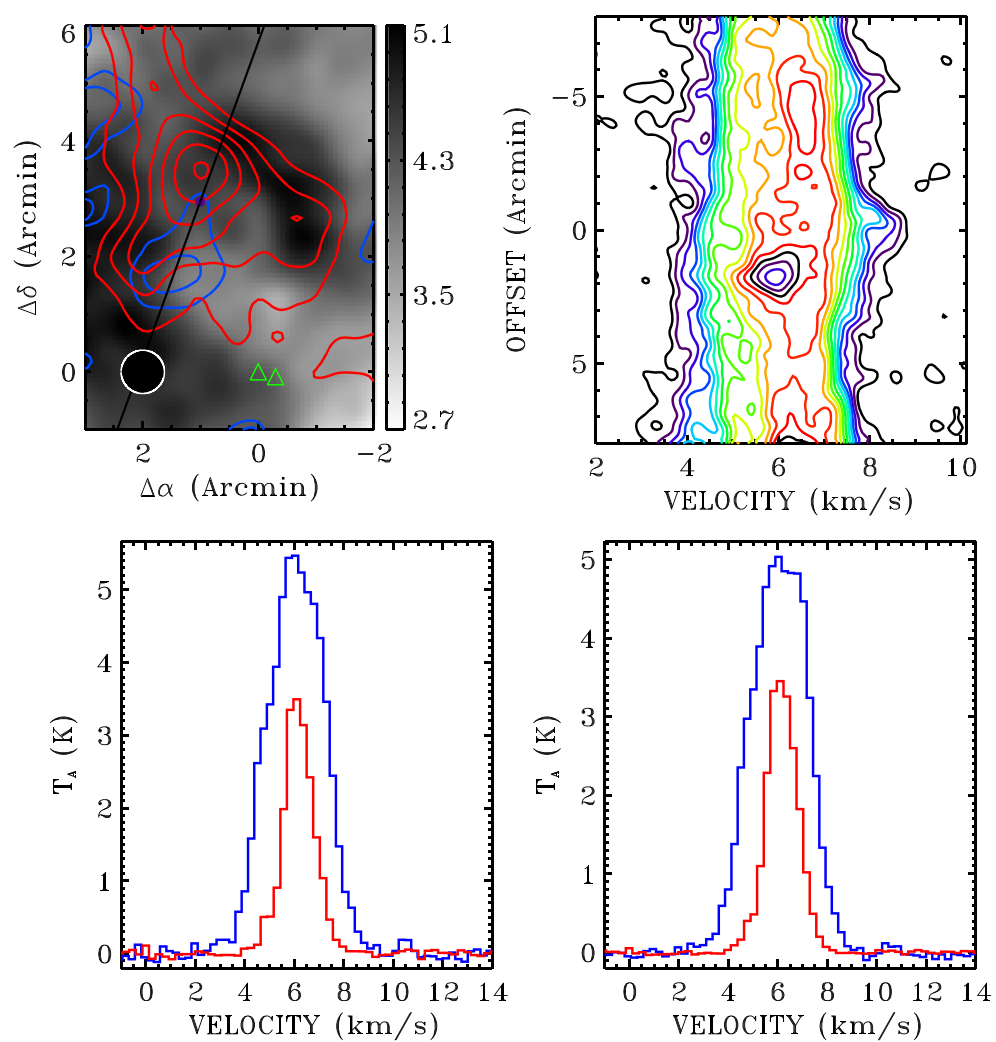

Figure 17. TMO 16 (SST 043231.7+242002). Same as Figure 2 except for the following. In the upper left panel, the integrated intervals for the blue lobe, red lobe, and grayscale images are 3-4.5 $\mathrm{km} \mathrm{s}^{-1}, 7.5-8.7 \mathrm{~km} \mathrm{~s}^{-1}$, and $5-7 \mathrm{~km} \mathrm{~s}^{-1}$, respectively. The blue and red contour levels are $80 \%, 90 \%$ and $50 \%, 60 \%, \ldots, 90 \%$ of their peak value, respectively. In the upper right panel, the position angle is $20^{\circ}$ and the contour levels are $0.4-1.6 \mathrm{~K}$ by $0.3 \mathrm{~K}, 2-7 \mathrm{~K}$ by $0.5 \mathrm{~K}$. 

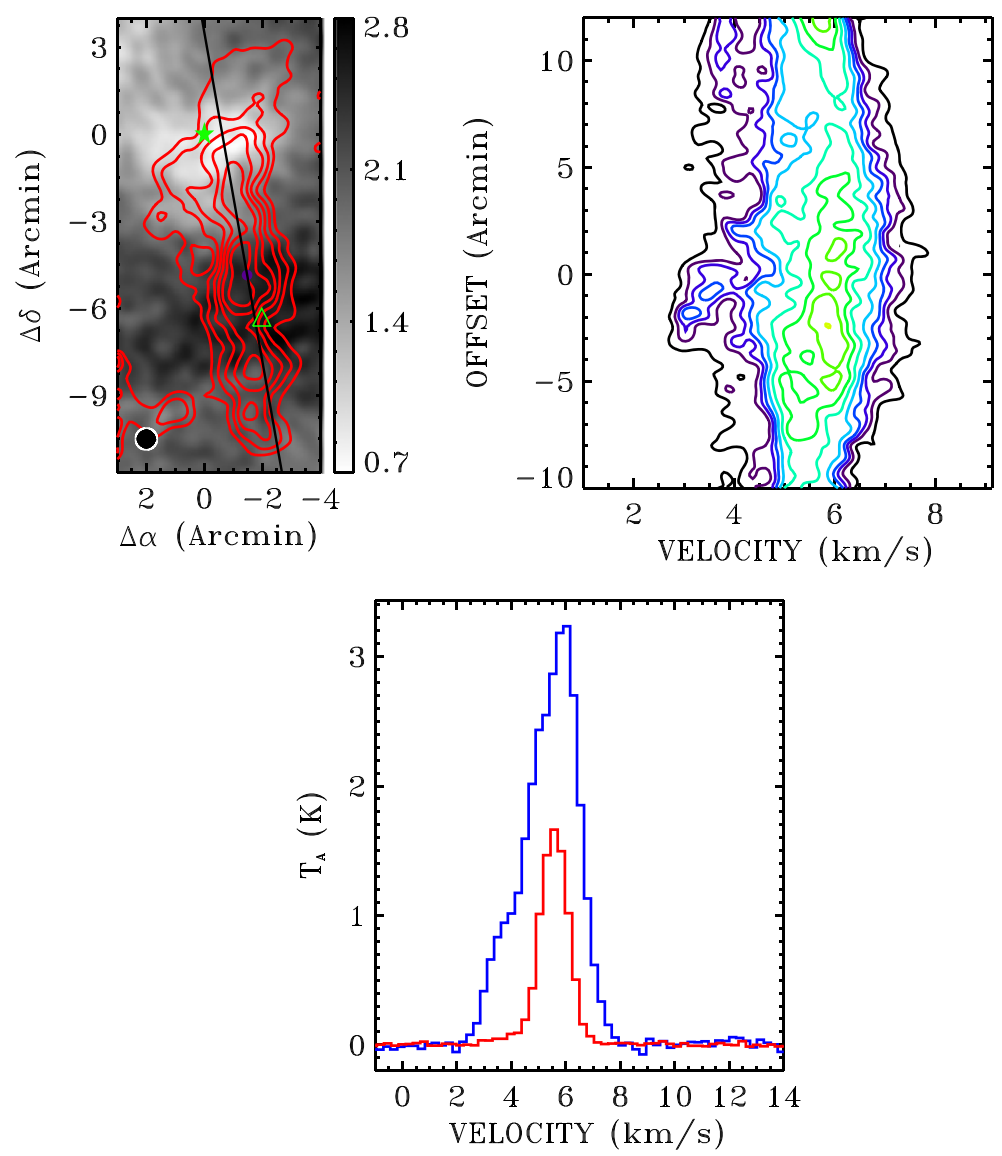

Figure 18. TMO 17 (SST 043232.0+225726). Same as Figure 3 except for the following. In the upper left panel, the integrated intervals for the red lobe and grayscale images are $6.8-7.8 \mathrm{~km} \mathrm{~s}^{-1}$ and $4.8-6.3 \mathrm{~km} \mathrm{~s}^{-1}$, respectively. The red contour levels are $40 \%, 50 \%, \ldots, 90 \%$ of the peak value. In the upper right panel, the position angle is $170^{\circ}$ and the contour levels are $0.4-1.6 \mathrm{~K}$ by $0.3 \mathrm{~K}, 2-7 \mathrm{~K}$ by $0.5 \mathrm{~K}$.
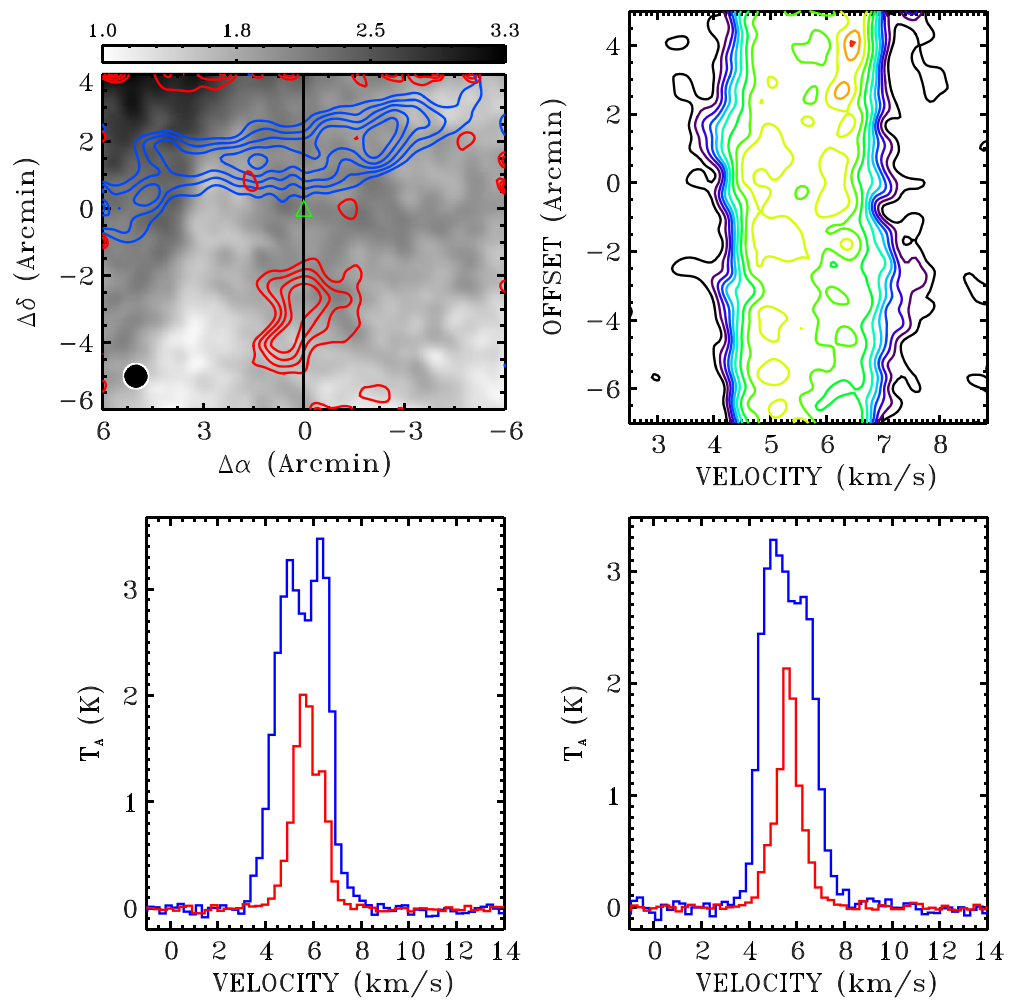

Figure 19. TMO_18 (SST 043243.0+255231). Same as Figure 2 except for the following. In the upper left panel, the integrated intervals for the blue lobe, red lobe, and grayscale image are 3.2-4 $\mathrm{km} \mathrm{s}^{-1}, 7-8 \mathrm{~km} \mathrm{~s}^{-1}$, and 5-6.5 $\mathrm{km} \mathrm{s}^{-1}$, respectively. The blue and red contour levels are $50 \%, 60 \%, \ldots, 90 \%$ and $60 \%, 70 \%, \ldots, 90 \%$ of their peak value, respectively. In the upper right panel, the position angle is $0^{\circ}$ and the contour levels are $0.4-1.6 \mathrm{~K}$ by $0.3 \mathrm{~K}, 2-4 \mathrm{~K}$ by $0.5 \mathrm{~K}$. 

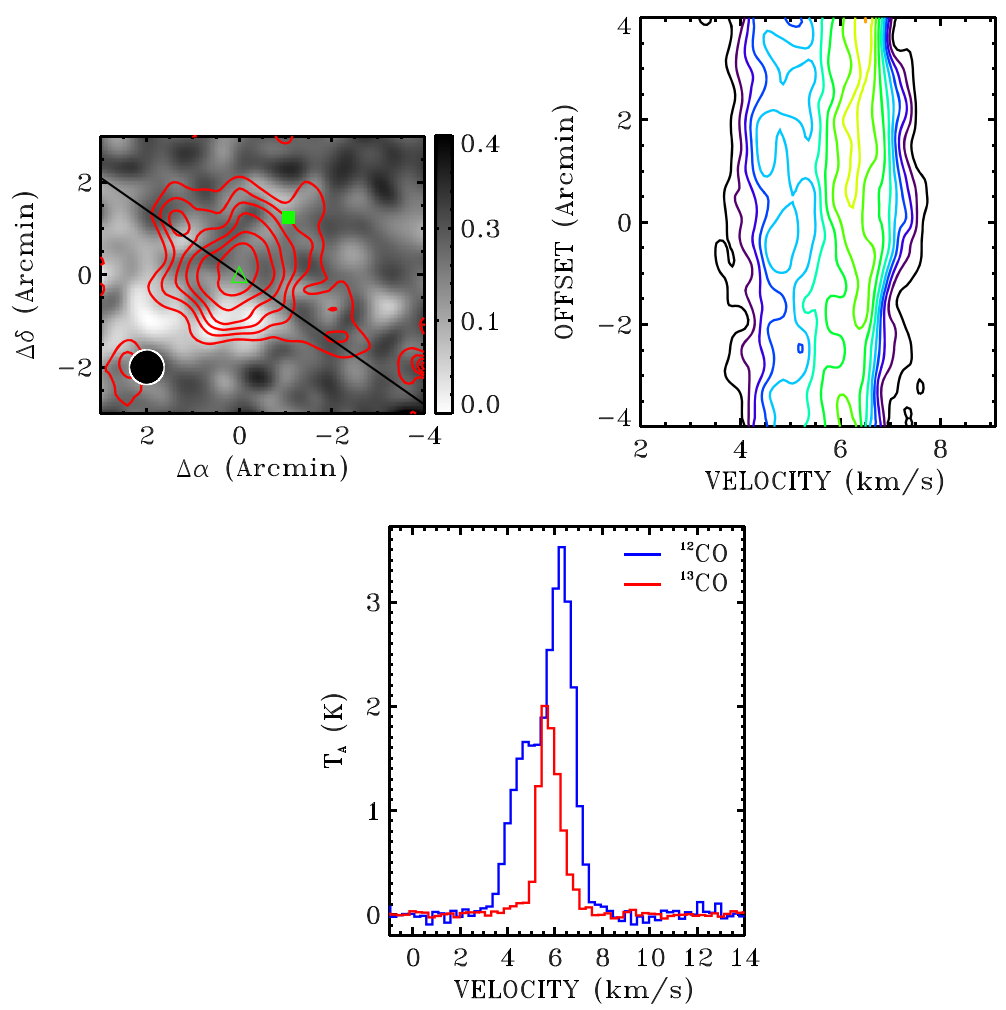

Figure 20. TMO 19 (SST 043415.2+225030). Same as Figure 3 except for the following. In the upper left panel, the integrated intervals for the red lobe and grayscale images are $7-8.5 \mathrm{~km} \mathrm{~s}^{-1}$ and $6-6.5 \mathrm{~km} \mathrm{~s}^{-1}$, respectively. The red contour levels are $40 \%, 50 \%, \ldots, 90 \%$ of the peak value. In the upper right panel, the position angle is $125^{\circ}$.
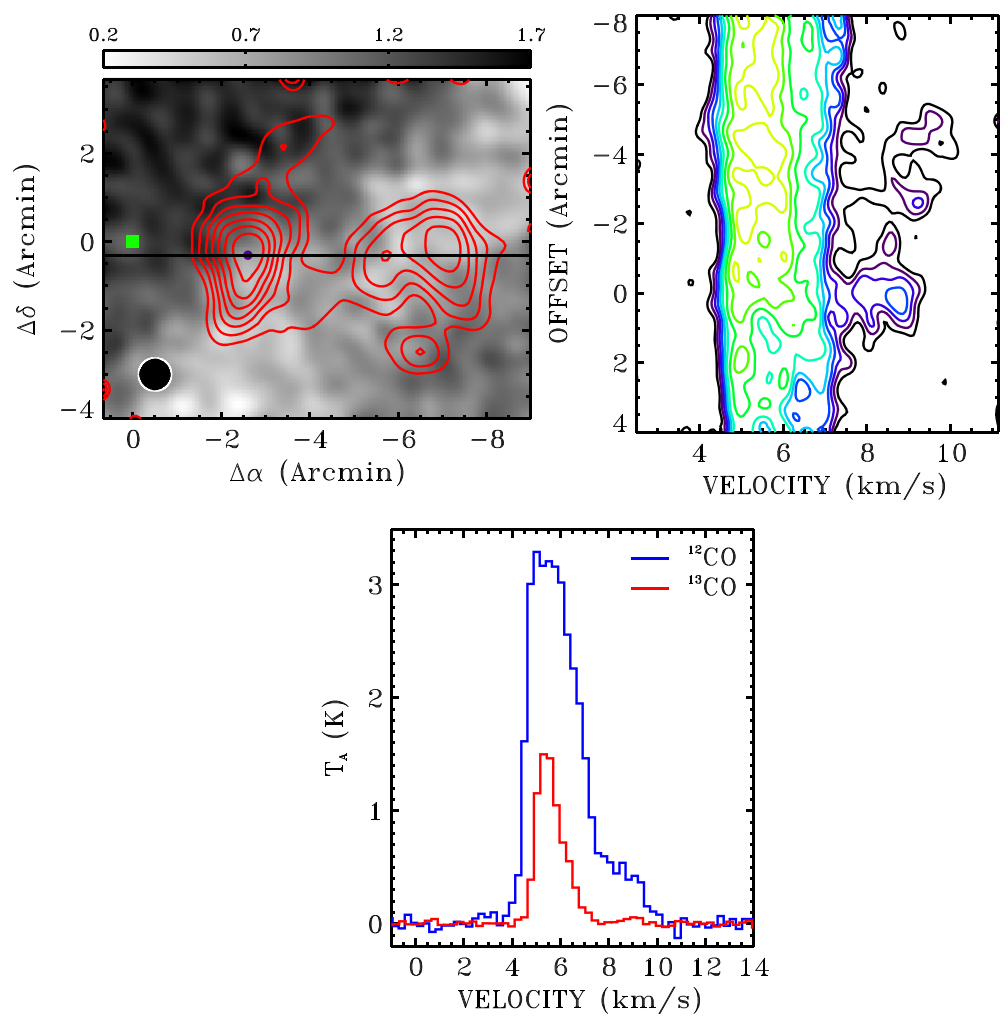

Figure 21. TMO 20 (SST 043724.8+270919). Same as Figure 3 except for the following. In the upper left panel, the integrated intervals for the red lobe and grayscale images are $8-10.5 \mathrm{~km} \mathrm{~s}^{-1}$ and $5-7 \mathrm{~km} \mathrm{~s}^{-1}$, respectively. The red contour levels are $30 \%, 40 \%, \ldots, 90 \%$ of the peak value. In the upper right panel, the position angle is $90^{\circ}$ and the contour levels are $0.5-2 \mathrm{~K}$ by $0.3 \mathrm{~K}, 2.5-4 \mathrm{~K}$ by $0.5 \mathrm{~K}$. 

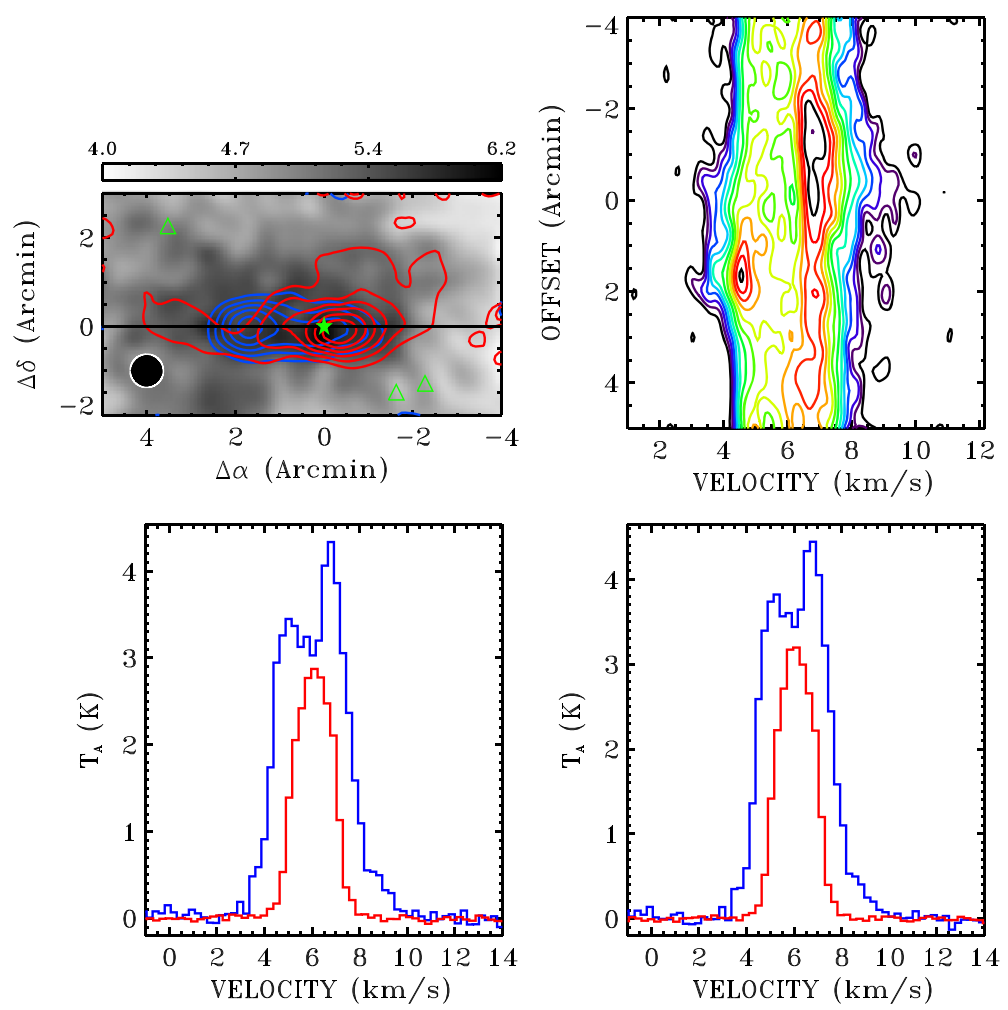

Figure 22. TMO 21 (SST 043953.9+260309). Same as Figure 2 except for the following. In the upper left panel, the integrated intervals for the blue lobe, red lobe, and grayscale images are $2-4 \mathrm{~km} \mathrm{~s}^{-1}, 8-10 \mathrm{~km} \mathrm{~s}^{-1}$, and $4.5-7.5 \mathrm{~km} \mathrm{~s}^{-1}$, respectively. The blue and red contour levels are $30 \%, 45 \%, \ldots, 90 \%$ and $40 \%, 50 \%, \ldots, 90 \%$ of their peak value, respectively. In the upper right panel, the position angle is $90^{\circ}$ and the contour levels are $2 \mathrm{~K}$ by $0.3 \mathrm{~K}, 2.5-6 \mathrm{~K}$ by $0.5 \mathrm{~K}$.
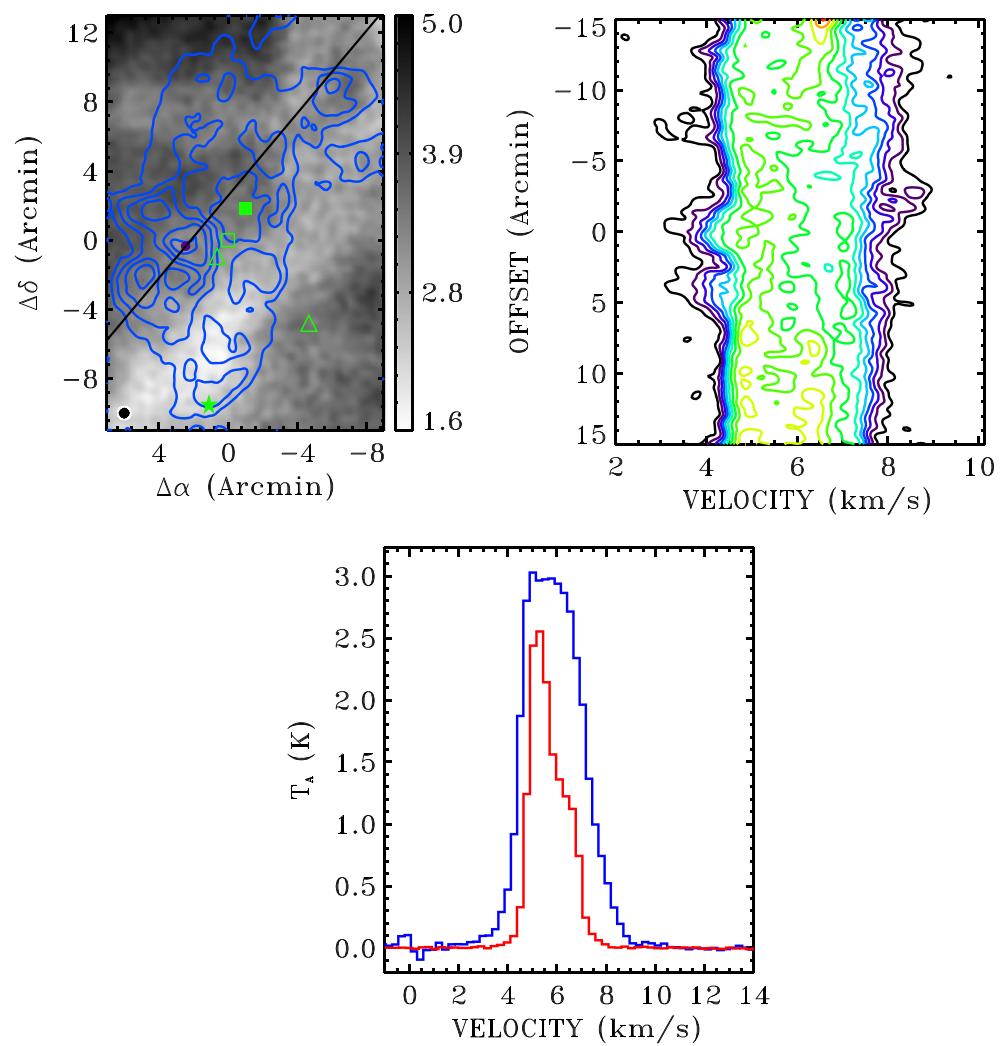

Figure 23. TMO 22 (SST 044108.2+255607). Same as Figure 6 except for the following. In the upper left panel, the integrated intervals for the blue lobe and grayscale images are $3-4.5 \mathrm{~km} \mathrm{~s}^{-1}$ and $4.6-7 \mathrm{~km} \mathrm{~s}^{-1}$, respectively. The blue contour levels are $35 \%, 50 \%, \ldots, 95 \%$ of the peak value. In the upper right panel, the contour levels are $0.4-1.6 \mathrm{~K}$ by $0.3 \mathrm{~K}, 2-4.5 \mathrm{~K}$ by $0.5 \mathrm{~K}$. 

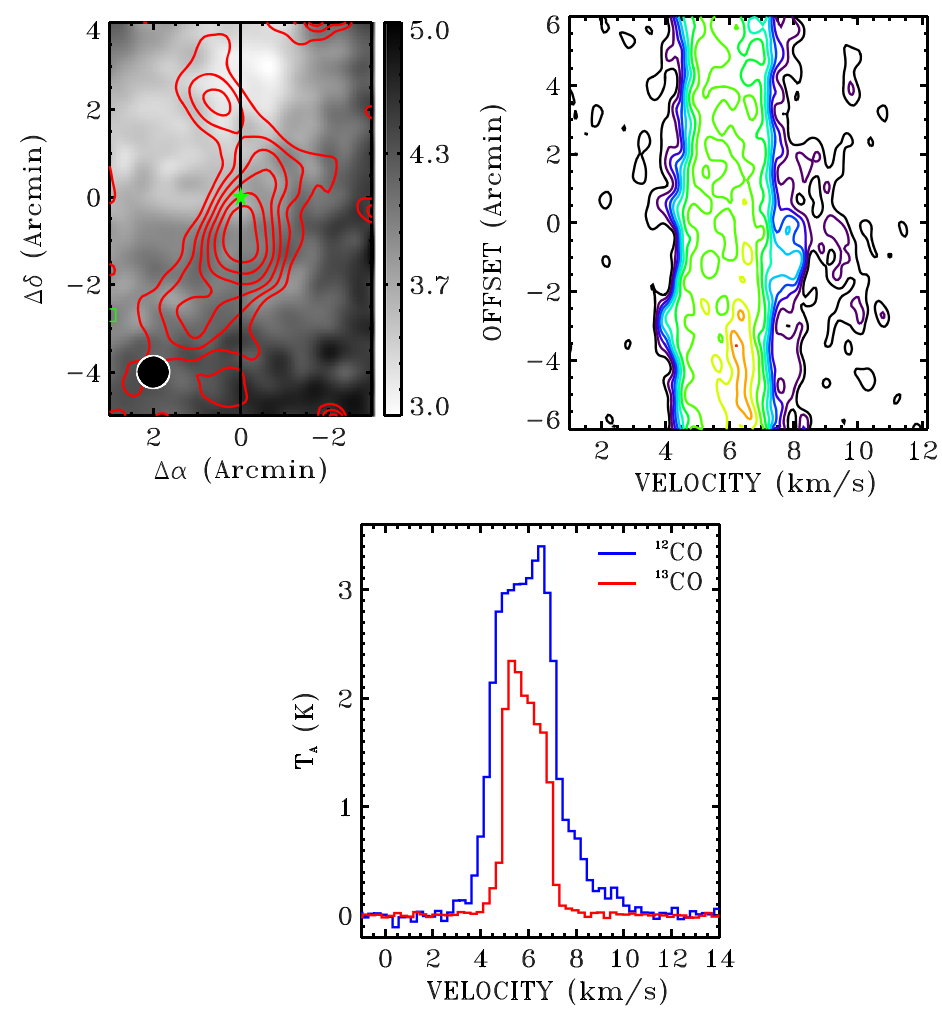

Figure 24. TMO 23 (SST 044112.6+254635). Same as Figure 3 except for the following. In the upper left panel, the integrated intervals for the red lobe and grayscale images are $7.5-10.5 \mathrm{~km} \mathrm{~s}^{-1}$ and $4.5-7 \mathrm{~km} \mathrm{~s}^{-1}$, respectively. The red contour levels are $40 \%, 50 \%, \ldots, 90 \%$ of the peak value. In the upper right panel, the position angle is $0^{\circ}$.
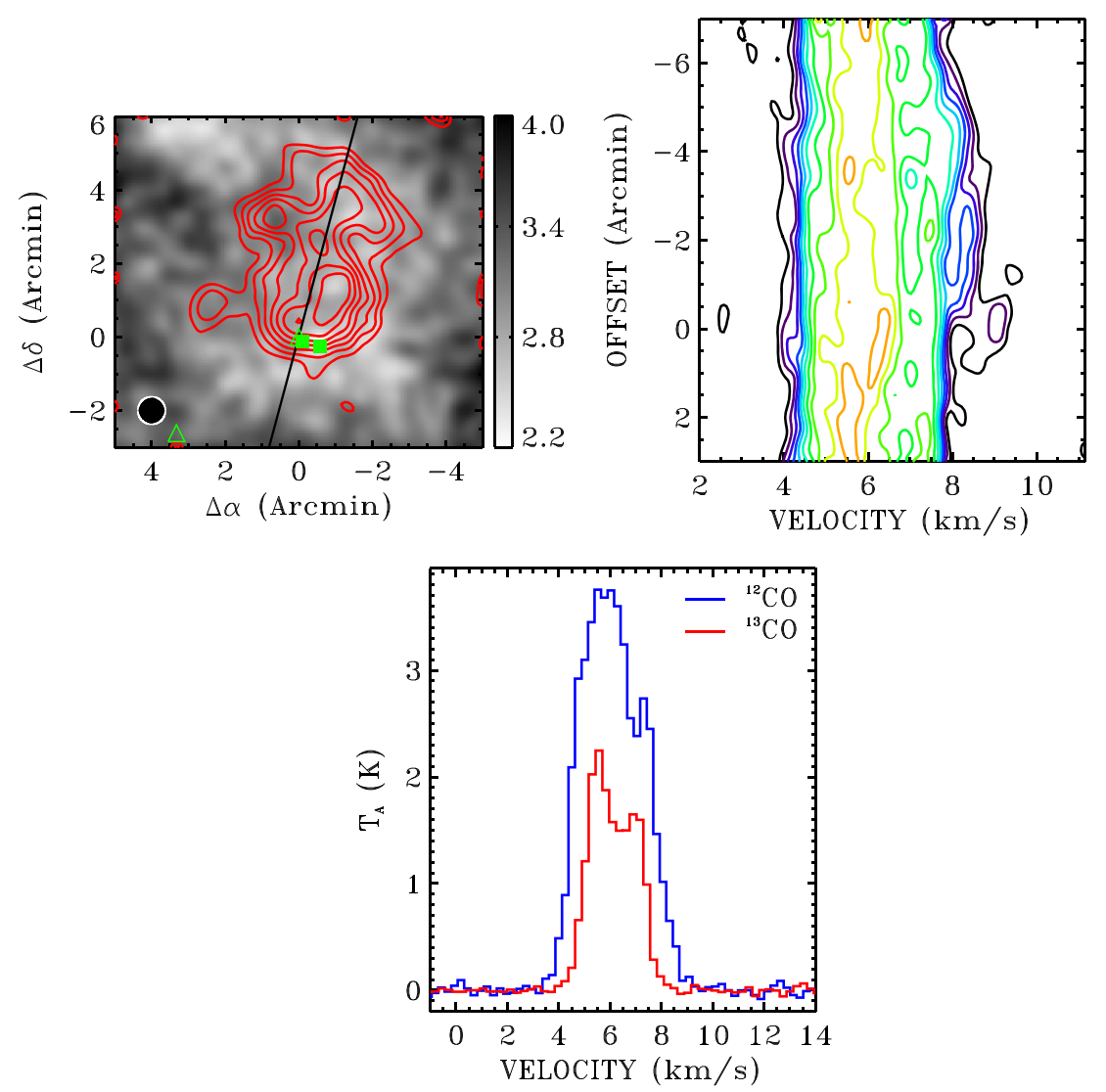

Figure 25. TMO_24 (SST 044207.7+252311). Same as Figure 3 except for the following. In the upper left panel, the integrated intervals for the red lobe and grayscale images are 8-9.5 $\mathrm{km} \mathrm{s}^{-1}$ and $5-7 \mathrm{~km} \mathrm{~s}^{-1}$, respectively. The red contour levels are $30 \%, 40 \%, \ldots, 90 \%$ of the peak value. In the upper right panel, the position angle is $15^{\circ}$ and the contour levels are $0.4-1.6 \mathrm{~K}$ by $0.3 \mathrm{~K}, 2-4.5 \mathrm{~K}$ by $0.5 \mathrm{~K}$. 

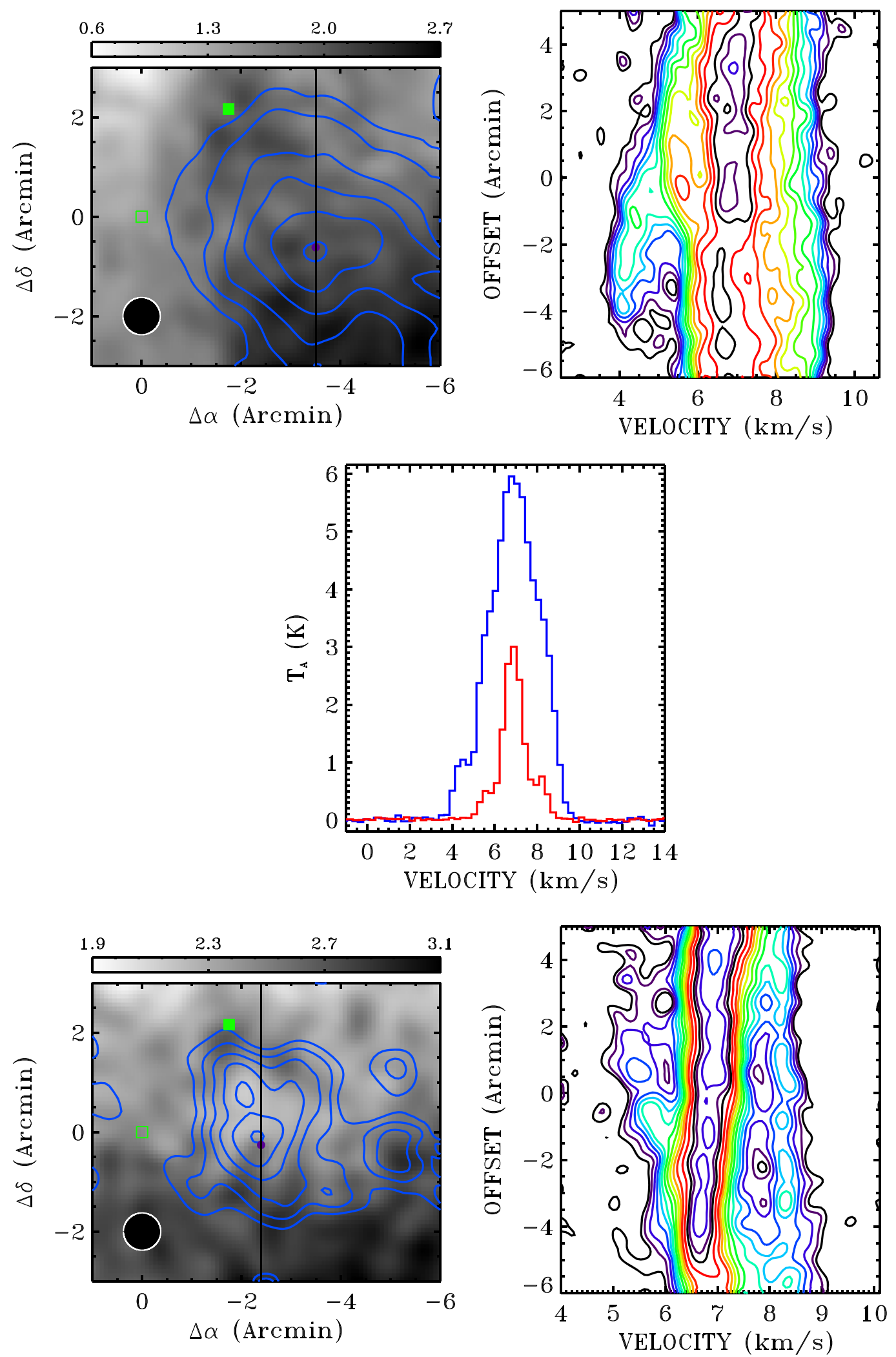

Figure 26. TMO_25 (SST 041858.1+281223). Upper left and upper right panels: same as the upper two panels of Figure 6 except for the following. In the upper left panel, the integrated intervals for the blue lobe and grayscale images are $3.5-5.5 \mathrm{~km} \mathrm{~s}^{-1}$ and $6.5-7.5 \mathrm{~km} \mathrm{~s}^{-1}$, respectively. The blue contour levels are $50 \%, 60 \%, \ldots$, $90 \%$ of the peak value. In the upper right panel, the position angle is $0^{\circ}$ and the contour levels are $0.5-2 \mathrm{~K}$ by $0.3 \mathrm{~K}, 2.5-6.5 \mathrm{~K}$ by $0.5 \mathrm{~K}$. Middle panel: same as the lower panel of Figure 6. Lower left panel: ${ }^{13} \mathrm{CO}$ integrated intensity map overlaid on the ${ }^{13} \mathrm{CO}$ grayscale image. The integrated intervals for the blue lobe and the grayscale images and the blue contour levels are the same as those in the upper left panel of this figure. Lower right panel: $\mathrm{P}-\mathrm{V}$ diagram of ${ }^{13} \mathrm{CO}$, through the same position angle in the upper left panel of this figure. Contour levels are $0.15-0.9 \mathrm{~K}$ by $0.15 \mathrm{~K}, 1.1-1.9 \mathrm{~K}$ by $0.2 \mathrm{~K}$, and $2.1-3.9 \mathrm{~K}$ by $0.3 \mathrm{~K}$. 

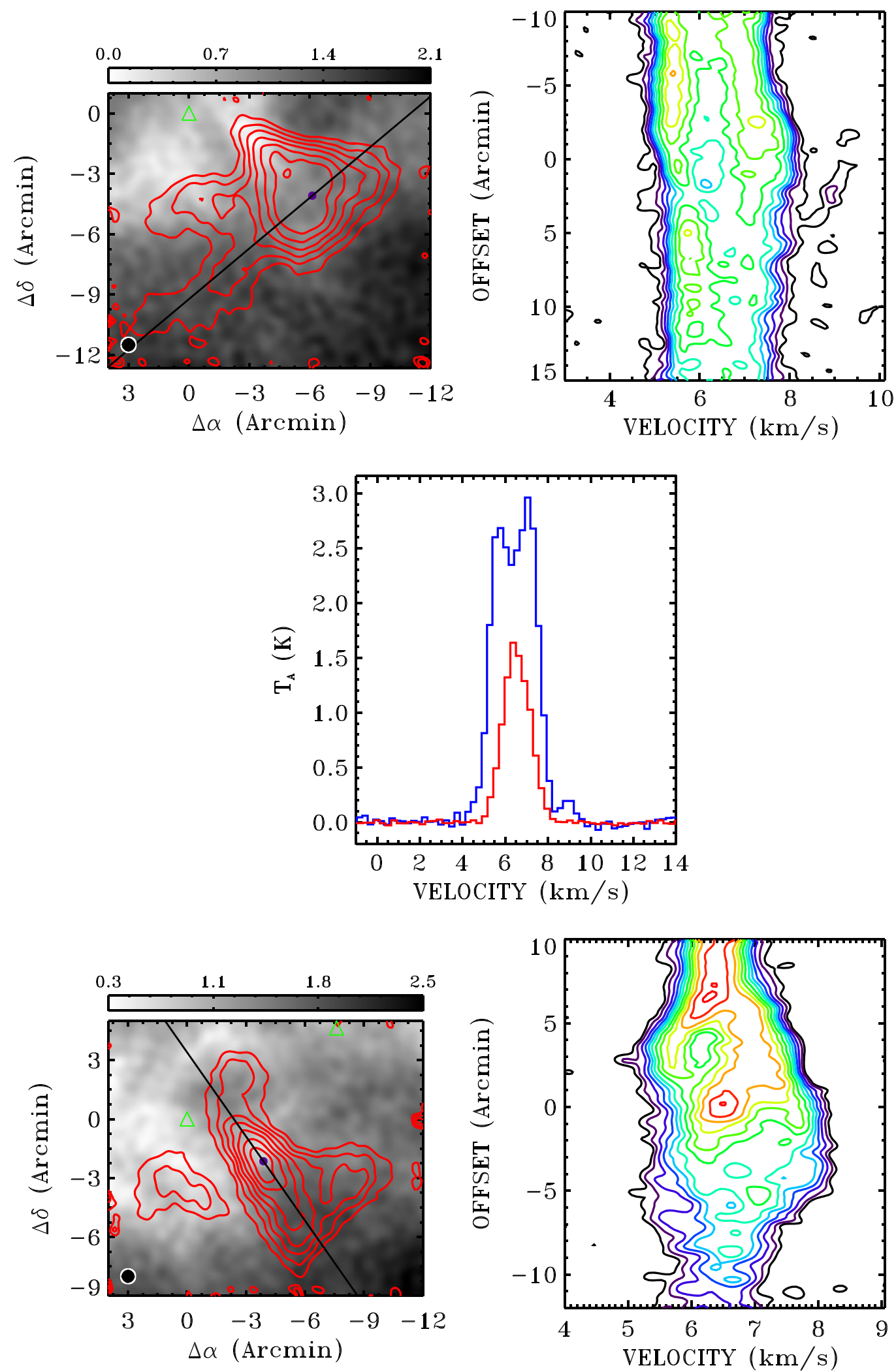

Figure 27. TMO_26 (SST 042318.2+264115). Upper left and upper right: same as the upper two panels of Figure 3 except for the following. In the upper left panel, the integrated intervals for the red lobe and grayscale images are $7.5-10 \mathrm{~km} \mathrm{~s}^{-1}$ and $6-7 \mathrm{~km} \mathrm{~s}^{-1}$, respectively. The red contour levels are $40 \%, 50 \%, \ldots, 90 \%$ of the peak value. In the upper right panel, the position angle is $50^{\circ}$. Middle panel: same as the lower panel of Figure 3 . Lower left panel: ${ }^{13} \mathrm{CO}$ integrated intensity map overlaid on the ${ }^{13} \mathrm{CO}$ gray-scale image. The integrated intervals for the red lobe and grayscale images are the same as those in the upper left panel of this figure. The red contour levels are $30 \%, 40 \%, \ldots, 90 \%$ of the peak value. Lower right panel: same as the lower right panel of Figure 26 except that the contour levels are $0.15-0.9 \mathrm{~K}$ by $0.15 \mathrm{~K}, 1.1-2.3 \mathrm{~K}$ by $0.2 \mathrm{~K}$. 

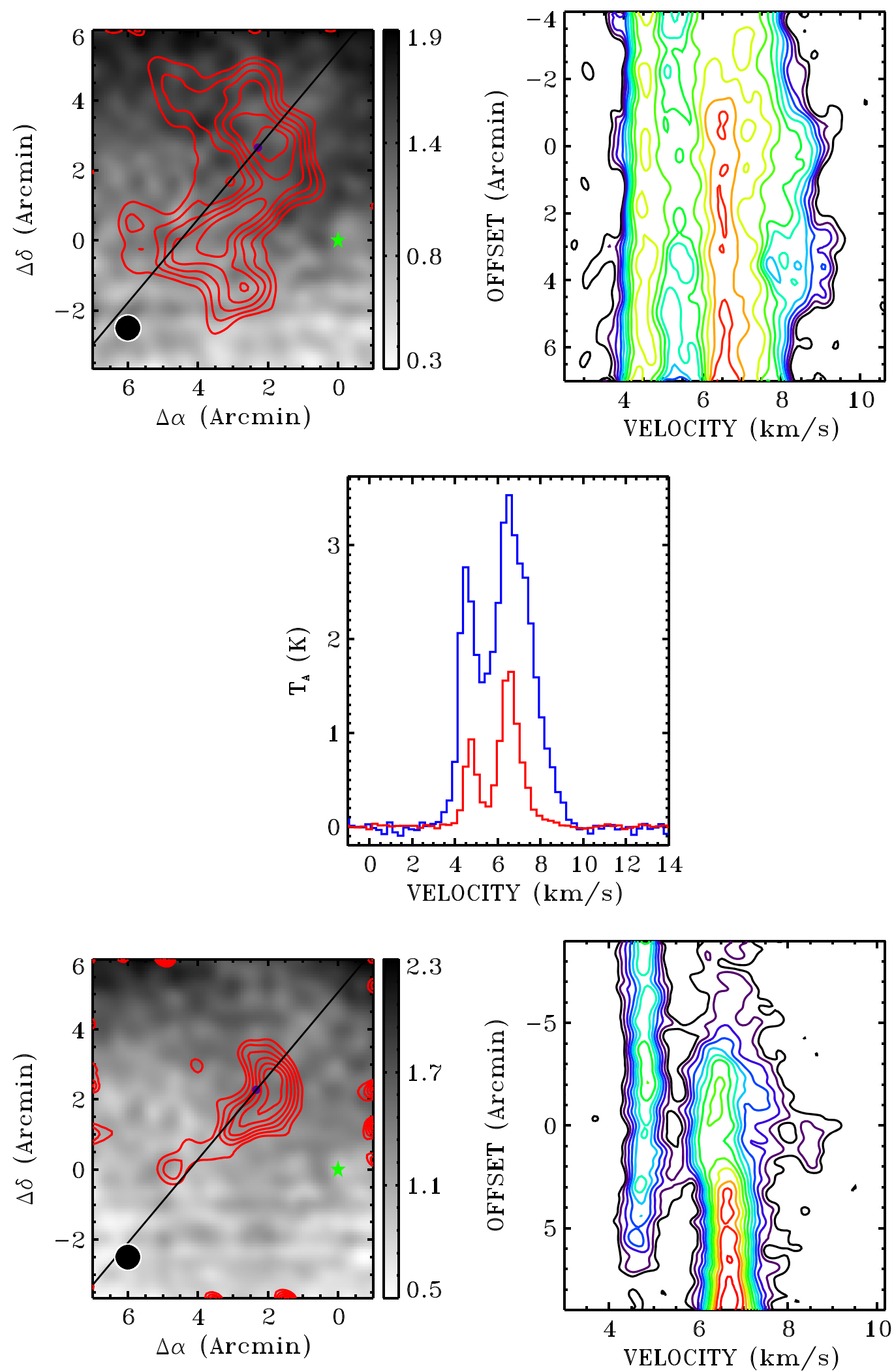

Figure 28. TMO_27 (SST 042656.2+244335). Same as Figure 27 except for the following. In the upper left panel, the integrated intervals for the red lobe and grayscale images are 8.3-9.7 $\mathrm{km} \mathrm{s}^{-1}$ and 6-7.5 $\mathrm{km} \mathrm{s}^{-1}$, respectively. In the upper right panel, the position angle is $40^{\circ}$. In the lower left panel, the integrated intervals for the red lobe and grayscale images and the red contour levels are the same as those in the upper left panel of this figure. In the lower right panel, the position angle is the same with the upper right panel of this figure and the contour levels are $0.15-0.9 \mathrm{~K}$ by $0.15 \mathrm{~K}, 1.1-2.1 \mathrm{~K}$ by $0.2 \mathrm{~K}$. 

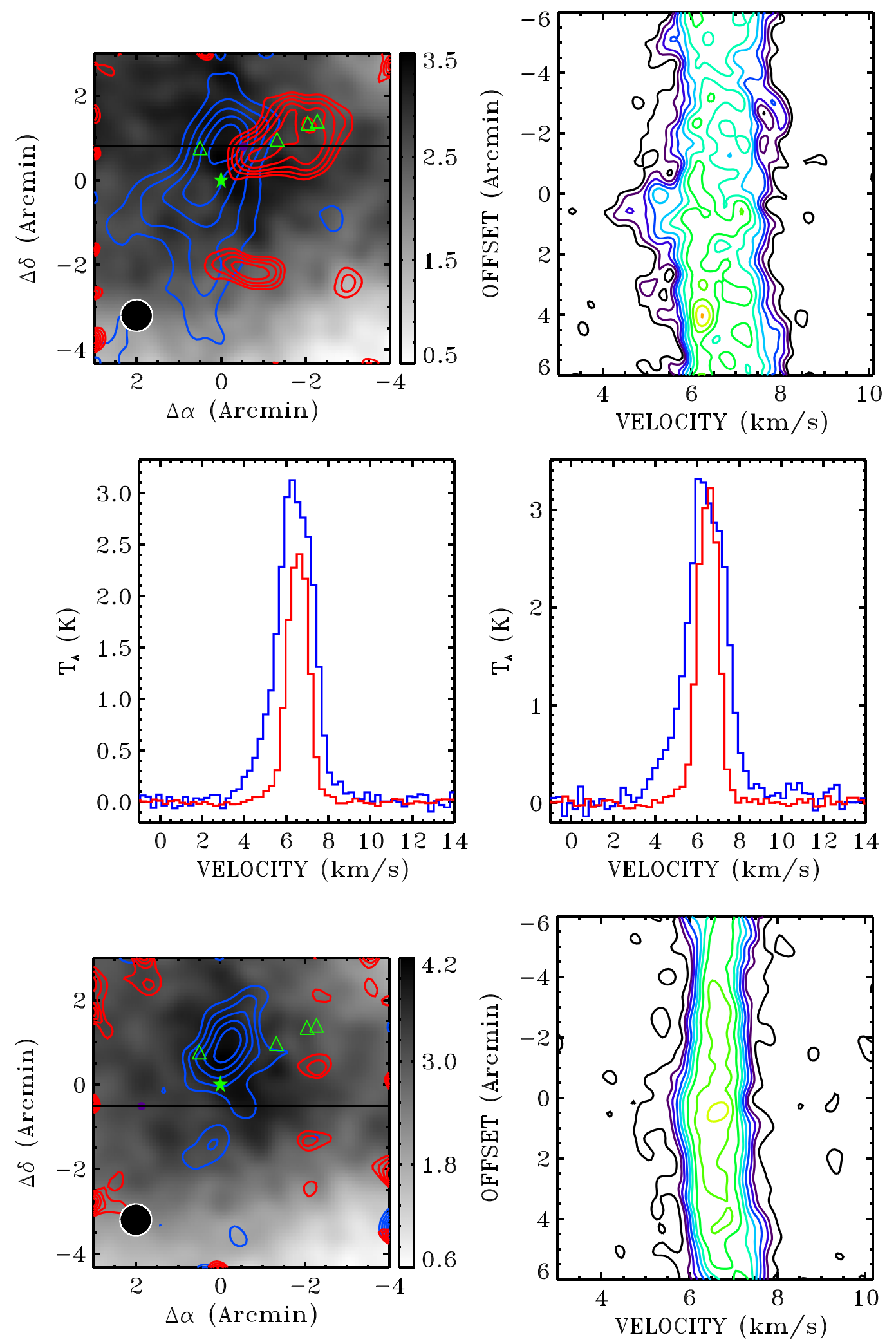

Figure 29. TMO_28 (SST 042702.6+260530). Upper left and upper right panels: same as the upper two panels of Figure 2 except for the following. In the upper left panel, the integrated intervals for the blue and red lobes are $3.4-5 \mathrm{~km} \mathrm{~s}^{-1}$ and $8-13 \mathrm{~km} \mathrm{~s}^{-1}$, respectively. The blue and red contour levels are both $50 \%, 60 \%, \ldots, 90 \%$ of their peak value. In the upper right panel, the position angle is $90^{\circ}$ and the contour levels are $0.7-1.6 \mathrm{~K}$ by $0.3 \mathrm{~K}, 2-4.5 \mathrm{~K}$ by $0.5 \mathrm{~K}$. Middle left and middle right panels: same as the lower two panels of Figure 2. Lower left panel: ${ }^{13} \mathrm{CO}$ integrated intensity map overlaid on the ${ }^{13} \mathrm{CO}$ grayscale image. The integrated intervals for the blue lobe, red lobe, and grayscale image, and the blue and red contour levels are the same as those in the upper left panel of this figure. Lower right: same as the lower right panel of Figure 26 except that the position angle is $90^{\circ}$ and the contour levels are $0.2-2 \mathrm{~K}$ by $0.3 \mathrm{~K}, 2.5-4.5 \mathrm{~K}$ by $0.5 \mathrm{~K}$. 

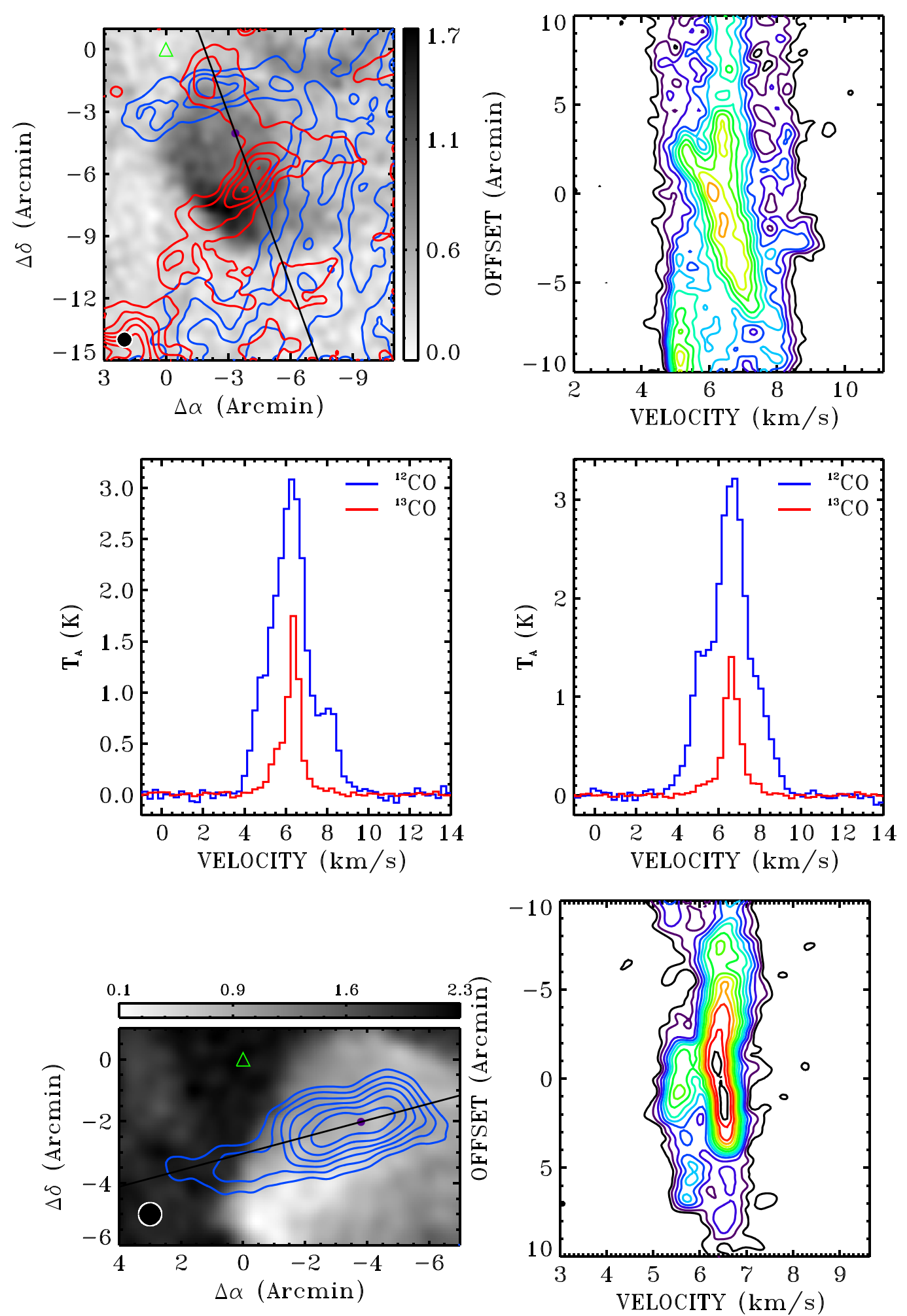

Figure 30. TMO 29 (SST 042702.8+254222). Same as Figure 29 except for the following. In the upper left panel, the integrated intervals for the blue lobe, red lobe, and grayscale images are $3.5-5.5 \mathrm{~km} \mathrm{~s}^{-1}, 8-9.5 \mathrm{~km} \mathrm{~s}^{-1}$, and $6-7 \mathrm{~km} \mathrm{~s}^{-1}$, respectively. The blue and red contour levels are $60 \%, 70 \%, \ldots, 90 \%$ and $30 \%, 40 \%, \ldots, 90 \%$ of their peak value, respectively. In the upper right panel, the position angle is $160^{\circ}$ and the contour levels are $0.4-1.6 \mathrm{~K}$ by $0.3 \mathrm{~K}, 2-4.5 \mathrm{~K}$ by $0.5 \mathrm{~K}$. In the lower left panel, the integrated interval for the blue lobe is $4.5-5.9 \mathrm{~km} \mathrm{~s}^{-1}$. The blue levels are $40 \%, 50 \%, \ldots, 90 \%$ of the peak value. In the lower right panel, the position angle is $75^{\circ}$ and the contour levels are $0.15-0.9 \mathrm{~K}$ by $0.15 \mathrm{~K}, 1.1-1.9 \mathrm{~K}$ by $0.2 \mathrm{~K}$, and $2.2-2.8 \mathrm{~K}$ by $0.3 \mathrm{~K}$. 

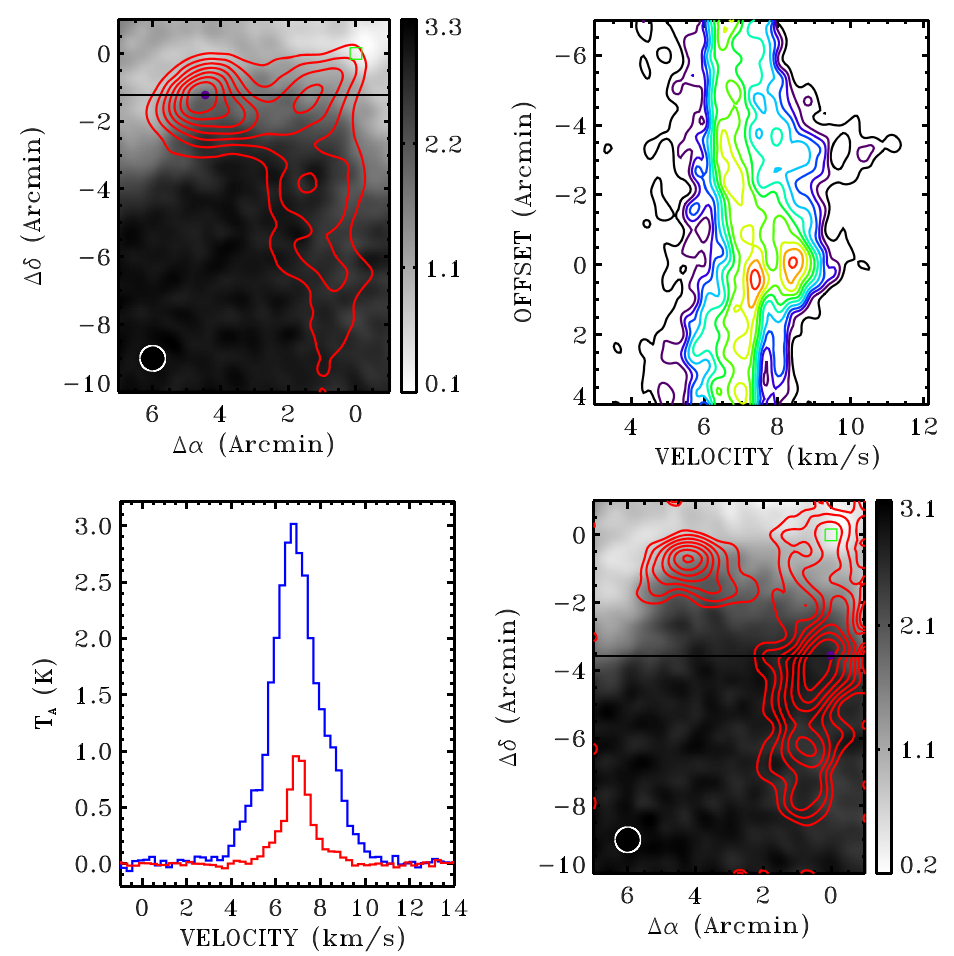

Figure 31. TMO_30 (SST 042757.3+261918). Same as Figure 14 except for the following. In the upper left panel, the integrated intervals for the red lobe and grayscale images are $8-10.5 \mathrm{~km} \mathrm{~s}^{-1}$ and $6-7.5 \mathrm{~km} \mathrm{~s}^{-1}$, respectively. The red contour levels are $30 \%, 40 \%, \ldots, 90 \%$ of the peak value. In the upper right panel, the position angle is $90^{\circ}$ and the contour levels are $0.4-1.6 \mathrm{~K}$ by $0.3 \mathrm{~K}, 2-7 \mathrm{~K}$ by $0.5 \mathrm{~K}$.
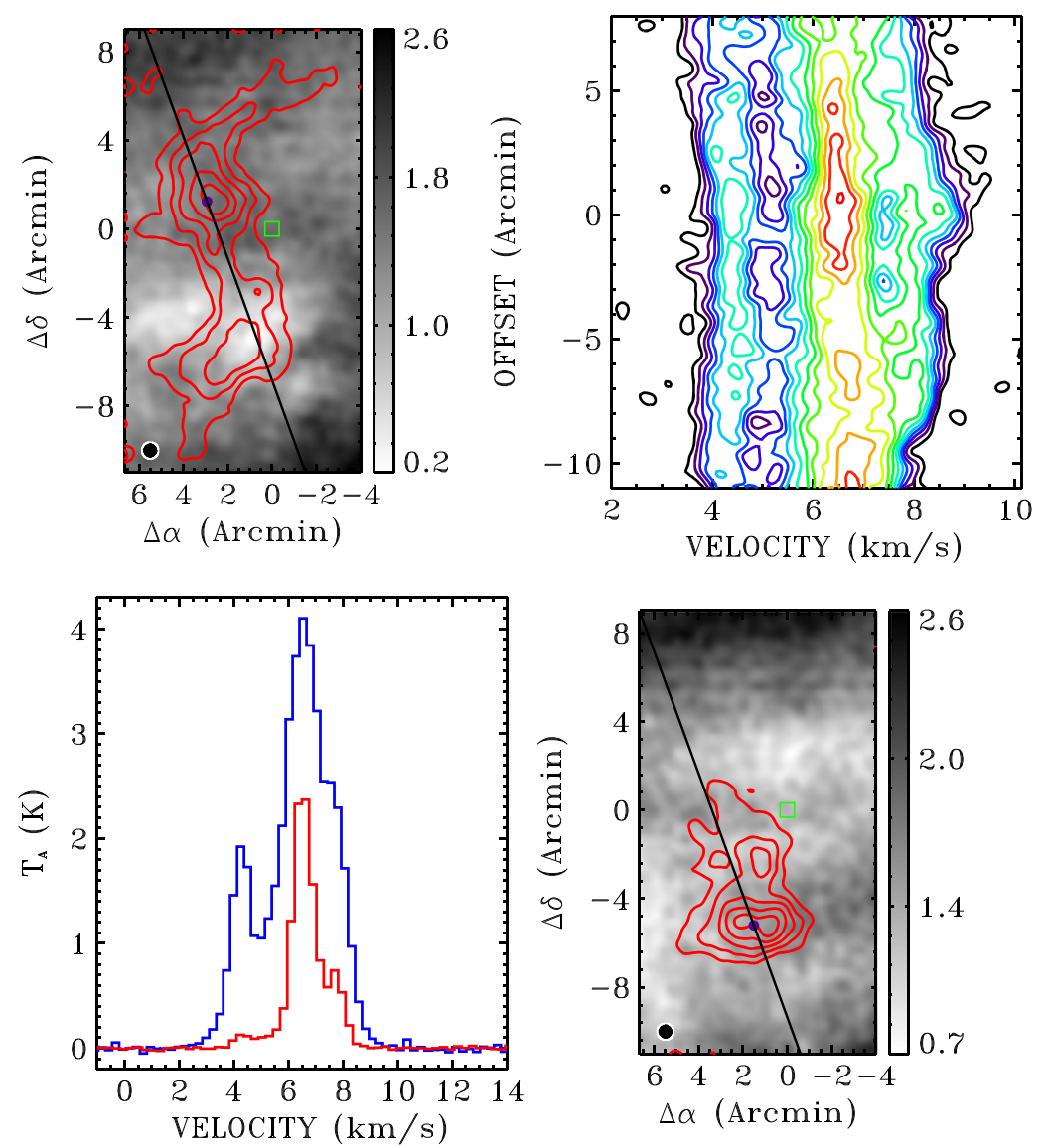

Figure 32. TMO 31 (SST 042810.4+243553). Same as Figure 14 except for the following. In the upper left panel, the integrated intervals for the red lobe and the grayscale image are $8-9.5 \mathrm{~km} \mathrm{~s}^{-1}$ and $6-7 \mathrm{~km} \mathrm{~s}^{-1}$, respectively. The red contour levels are $30 \%, 45 \%, \ldots, 90 \%$ of the peak value. In the upper right panel, the position angle is $160^{\circ}$ and the contour levels are $0.4-1.6 \mathrm{~K}$ by $0.3 \mathrm{~K}, 2-5 \mathrm{~K}$ by $0.5 \mathrm{~K}$. 

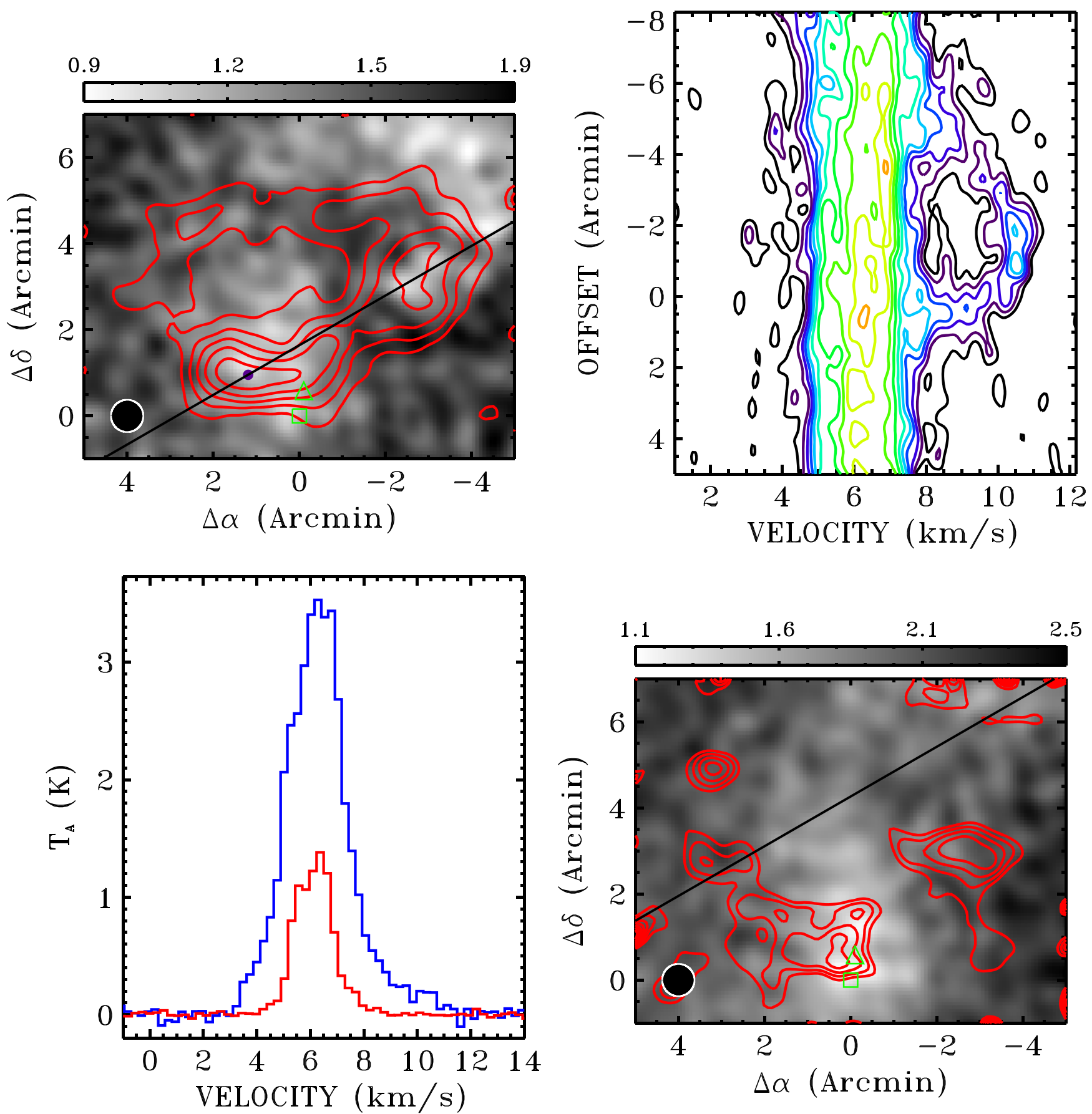

Figure 33. TMO 32 (SST 043051.7+244147). Same as Figure 14 except for the following. In the upper left panel, the integrated intervals for the red lobe and grayscale images are $8-11.3 \mathrm{~km} \mathrm{~s}^{-1}$ and $5.5-7 \mathrm{~km} \mathrm{~s}^{-1}$, respectively. The red contour levels are $30 \%, 45 \%, \ldots, 90 \%$ of the peak value. In the upper right panel, the position angle is $60^{\circ}$ and the contour levels are $0.4-1.6 \mathrm{~K}$ by $0.3 \mathrm{~K}, 2-7 \mathrm{~K}$ by $0.5 \mathrm{~K}$.

The mass, momentum, energy, and luminosity in Table 5 are only lower limits because we did not take into account the inclination and blending correction in the calculation. The mass should be multiplied by a factor of two due to blending. Assuming the average inclination angle of outflows is $57: 3$, the velocity and dynamic age should be scaled up by a factor of 1.9 and 0.64 , respectively. Combining the correction factors due to blending and inclination, the momentum, kinetic energy, and luminosity of outflows should be multiplied by a factor of 3.8, 6.8 , and 11, respectively. After correction, the total mass, momentum, energy, and luminosity of all outflows found in Taurus are approximately $15.4 M_{\odot}, 77 M_{\odot} \mathrm{km} \mathrm{s}^{-1}, 3.9 \times 10^{45}$ erg, and $1.3 \times 10^{33} \mathrm{erg} \mathrm{s}^{-1}$, respectively. The totals of the previously known outflows are about $8.6 M_{\odot}, 47 M_{\odot} \mathrm{km} \mathrm{s}^{-1}$, $2.6 \times 10^{45} \mathrm{erg}$, and $9.4 \times 10^{32} \mathrm{erg} \mathrm{s}^{-1}$, respectively. We found 1.8 times more outflowing mass, 1.6 times more momentum, and 1.5 times more energy from outflows injected into the Taurus molecular cloud than in previous studies. A high spatial dynamic range and systematic spectral line survey 

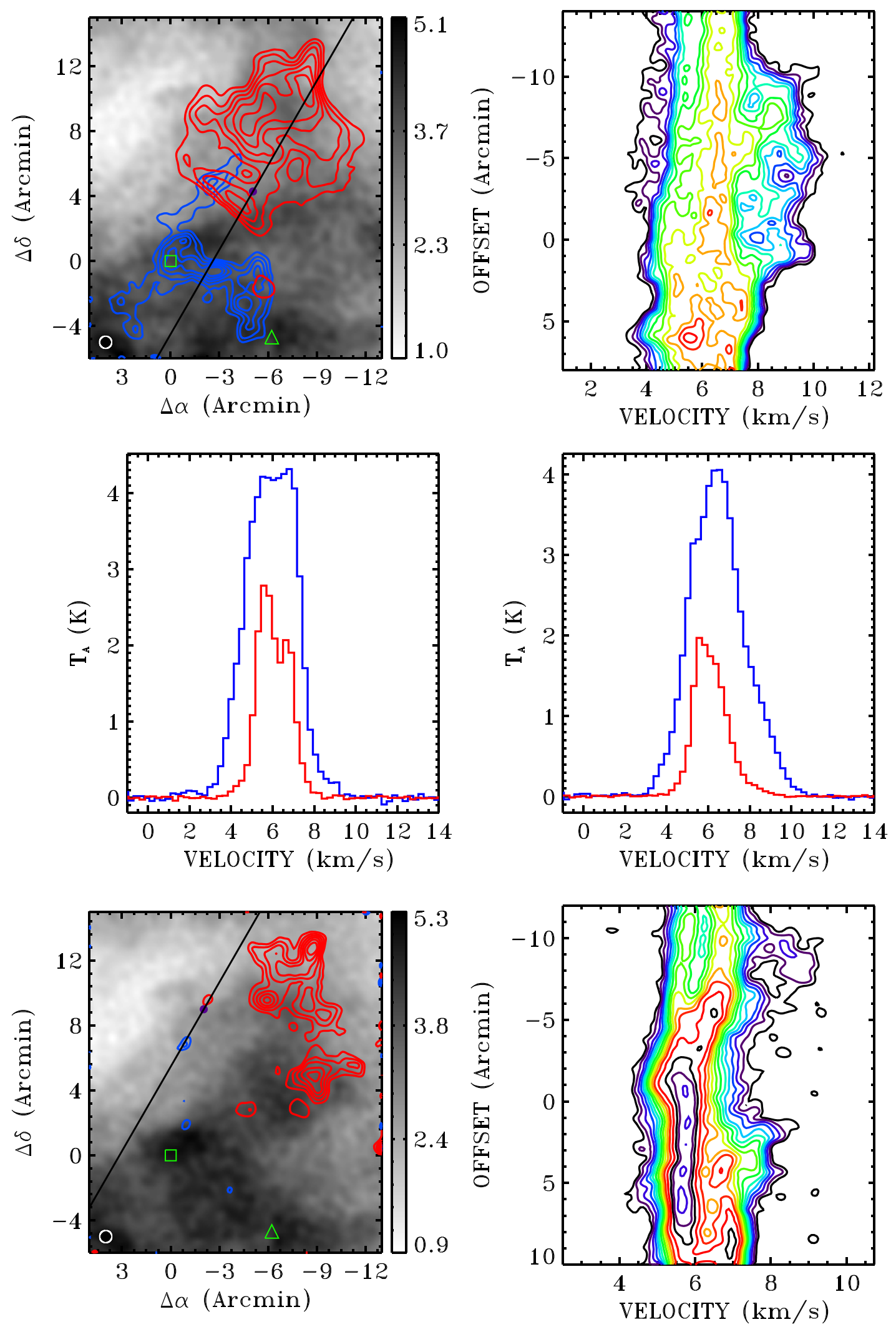

Figure 34. TMO_33 (SST 043215.4+242859). Same as Figure 29 except for the following. In the upper left panel, the integrated intervals for the blue lobe, red lobe, and grayscale images are $3-4 \mathrm{~km} \mathrm{~s}^{-1}, 8-10.5 \mathrm{~km} \mathrm{~s}^{-1}$, and $5-7 \mathrm{~km} \mathrm{~s}^{-1}$, respectively. In the upper right panel, the position angle is $30^{\circ}$ and the contour levels are $0.4-1.6 \mathrm{~K}$ by $0.3 \mathrm{~K}, 2-5.5 \mathrm{~K}$ by $0.5 \mathrm{~K}$. In the lower right panel, the contour levels are $0.15-0.9 \mathrm{~K}$ by $0.15 \mathrm{~K}, 1.1-3.2 \mathrm{~K}$ by $0.2 \mathrm{~K}$. 

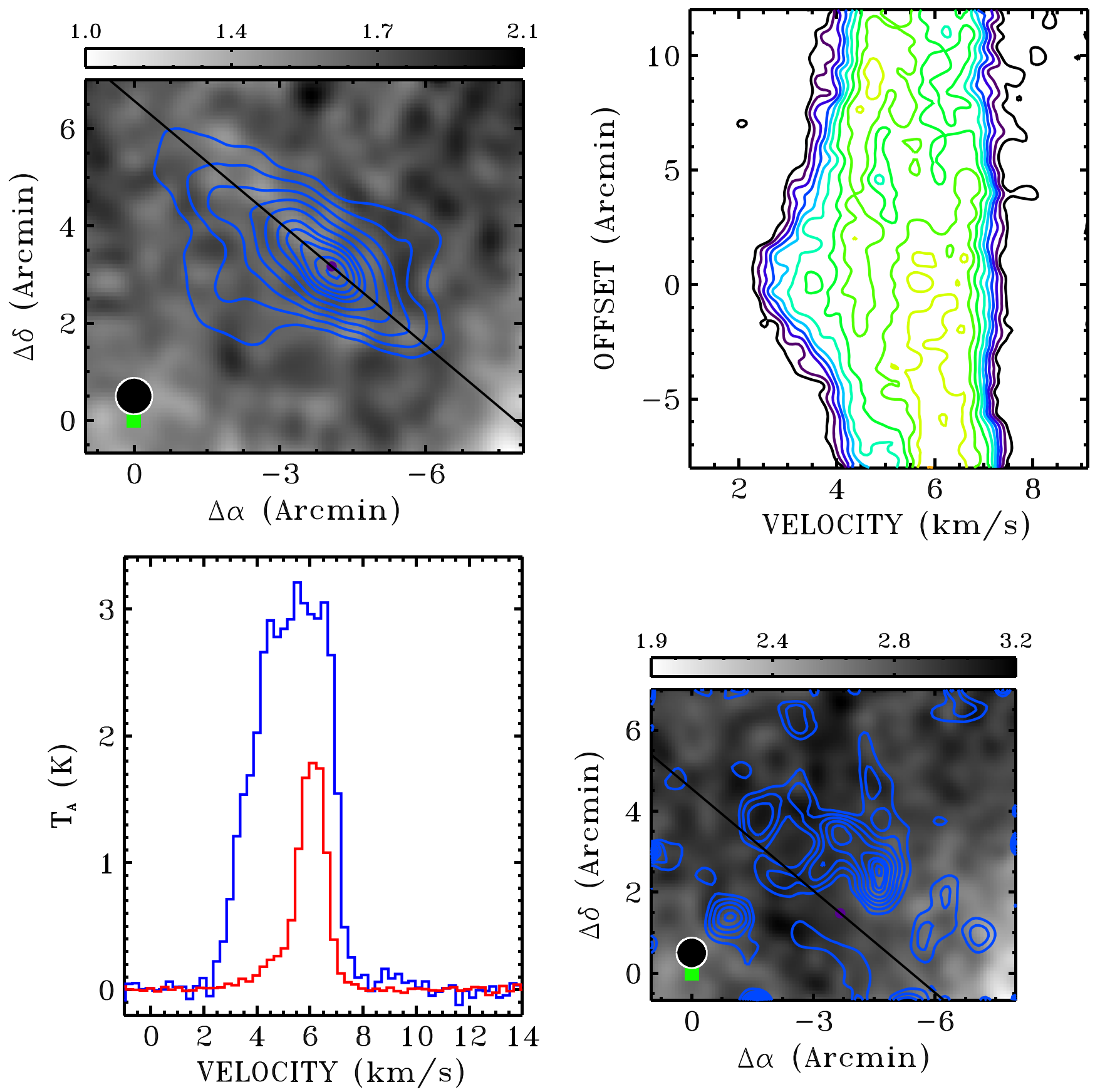

Figure 35. TMO 34 (SST 043307.8+261606). Upper left and upper right panels: same as the upper two panels of Figure 6 except for the following. In the upper left panel, the integrated intervals for the blue lobe and grayscale images are $2.3-3.5 \mathrm{~km} \mathrm{~s}^{-1}$ and $5.5-7 \mathrm{~km} \mathrm{~s}^{-1}$, respectively. The blue contour levels are $30 \%$, $40 \%, \ldots$, $90 \%$ of the peak value. In the upper right panel, the position angle is $130^{\circ}$ and the contour levels are $0.4-1.6 \mathrm{~K}$ by $0.3 \mathrm{~K}, 2-4 \mathrm{~K}$ by $0.5 \mathrm{~K}$. Lower left panel: same as the lower panel of Figure 6. Lower right panel: same as the lower left panel of Figure 26.

with good angular resolution is clearly necessary to obtain a more complete picture of the influence of outflows on their parent cloud.

\section{BUBBLES}

Following the method of identifying bubbles presented in Arce et al. (2011), we have identified 37 bubbles in the $\sim 100 \mathrm{deg}^{2}$ region of Taurus. The procedures for bubble searching, the morphology, and the physical parameters of bubbles are described in the following sections.

\subsection{The Procedures of Searching for Bubbles}

We undertook a blind search for bubbles using the FCRAO ${ }^{13} \mathrm{CO}$ data cube. The integrated intensity map, $\mathrm{P}-\mathrm{V}$ diagram, and channel maps of each bubble were examined. The detailed steps of the search were as follows.

1. We first searched for circular or arc-like (hereafter bubble-like) structures in the ${ }^{13} \mathrm{CO}$ data cube channel by channel through visual inspection. If there is a bubblelike structure in at least three contiguous channels, we considered it to be a bubble candidate. The approximate 

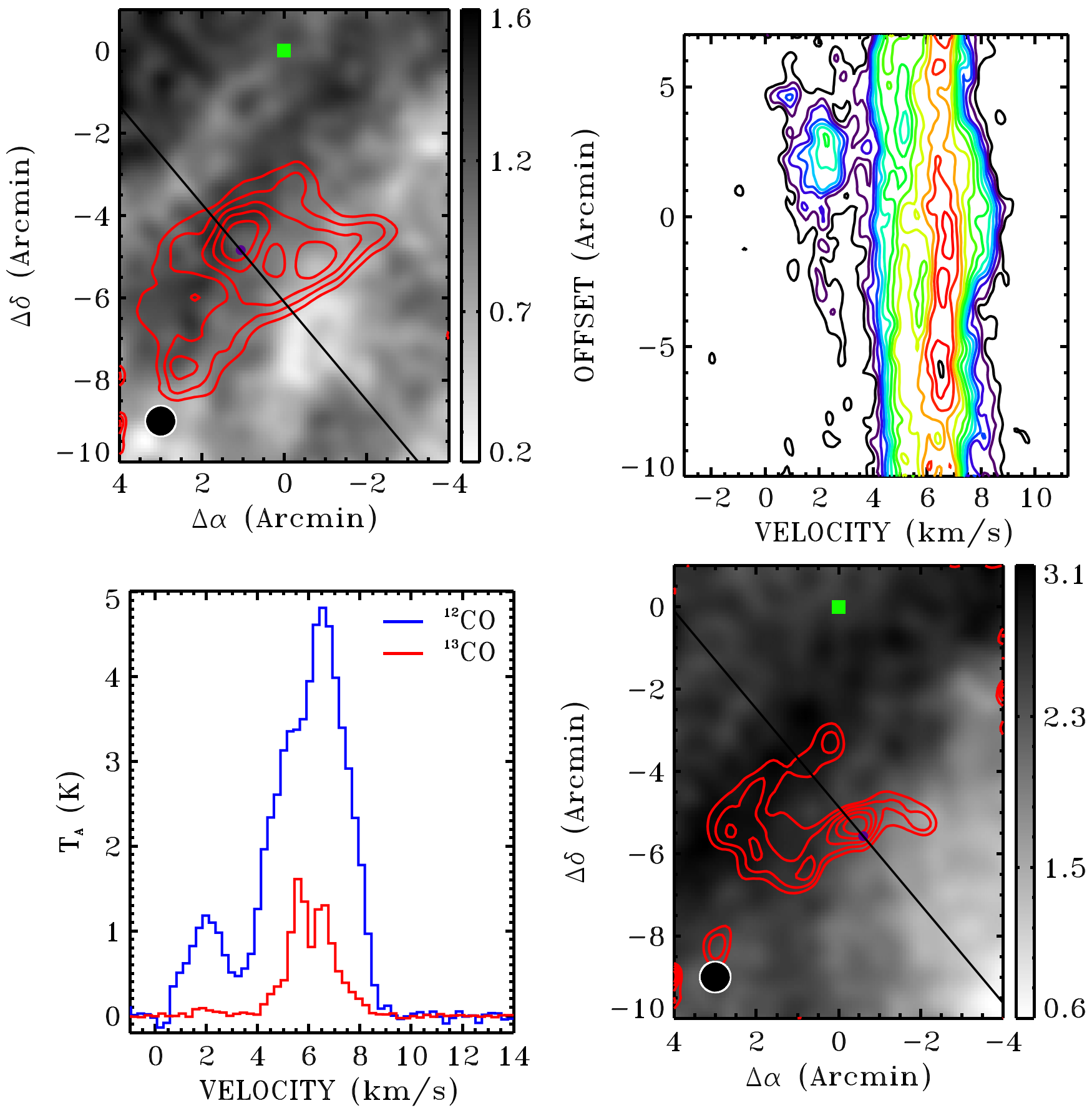

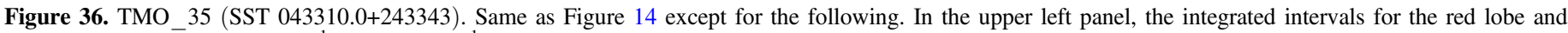

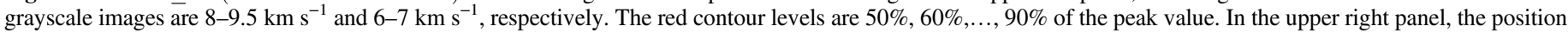
angle is $140^{\circ}$ and the contour levels are $0.4-1.6 \mathrm{~K}$ by $0.3 \mathrm{~K}, 2-5.5 \mathrm{~K}$ by $0.5 \mathrm{~K}$.

central position and radius of each candidate were recorded for further analysis. We also marked the channels where the bubble-like structure appears. With the marked channels, we obtained the expanding velocity interval of a bubble.

2. We plotted ${ }^{13} \mathrm{CO}$ contour maps around the central position of the bubble candidates with expanding velocity intervals.

3. We plotted $\mathrm{P}-\mathrm{V}$ diagrams in ${ }^{13} \mathrm{CO}$ through the central position of each candidate of every $15^{\circ}$ in position angle. We chose the one with the most obvious circular or "V" structure to show in the figures. The circular or "V" structure in the $\mathrm{P}-\mathrm{V}$ diagram is described in the expanding bubble model (Arce et al. 2011).

4. We plotted the ${ }^{13} \mathrm{CO}$ channel maps of each candidate to look over the variation of radius with velocity.

5. Finally, we fit a Gaussian profile to the azimuthally averaged profile of the ${ }^{13} \mathrm{CO}$ intensity of each candidate in the channel where the bubble morphology is most like a ring or arc. The radius of a bubble was obtained from the peak position of the fitted profile. 

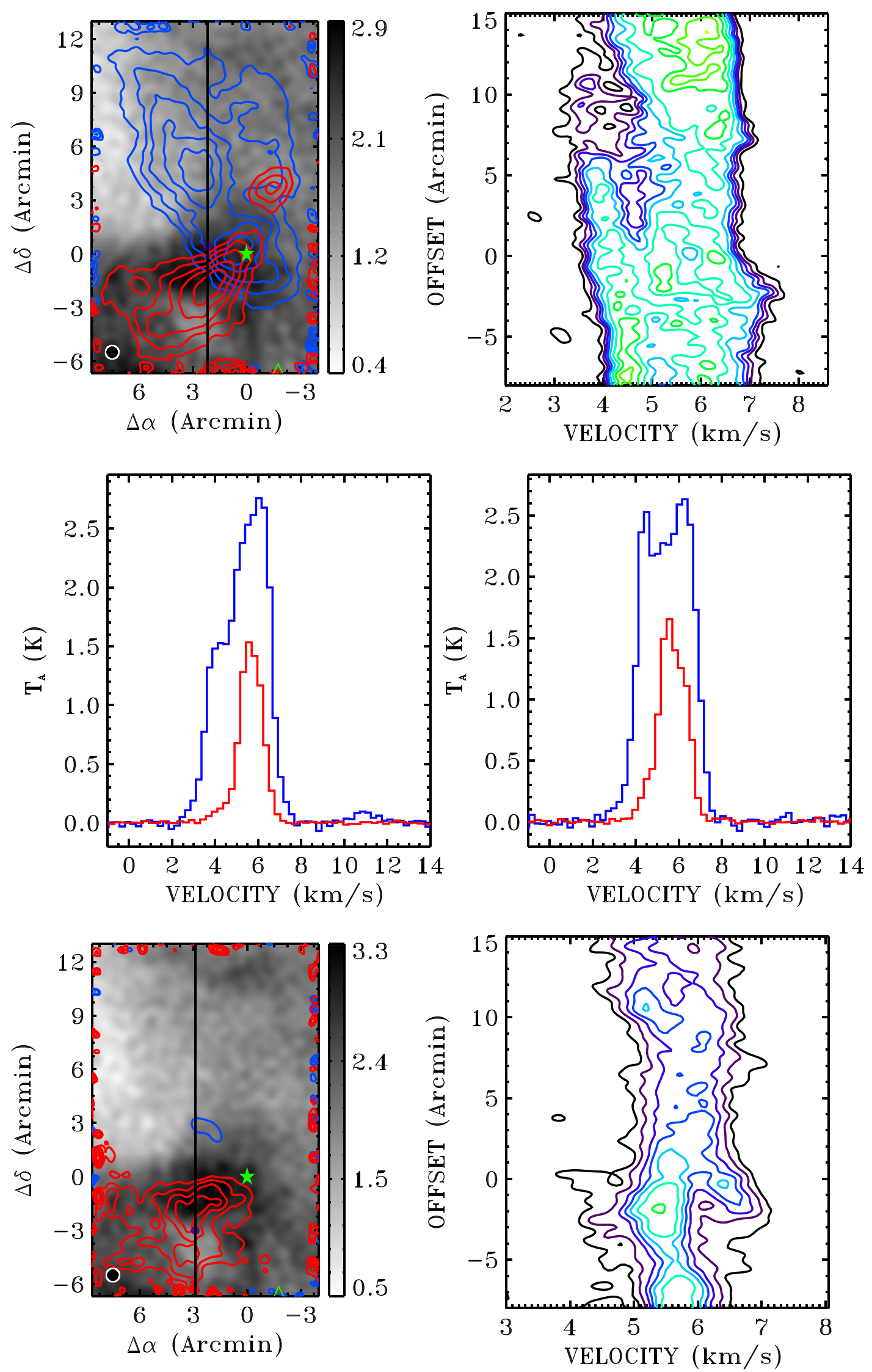

Figure 37. TMO 36 (SST 043316.5+225320). Same as Figure 29 except for the following. In the upper left panel, the integrated intervals for the blue lobe, red lobe, and grayscale images are 2-4 $\mathrm{km} \mathrm{s}^{-1}, 7-8 \mathrm{~km} \mathrm{~s}^{-1}$, and $4.5-6.5 \mathrm{~km} \mathrm{~s}^{-1}$, respectively. In the upper right panel, the position angle is $0^{\circ}$ and the contour levels are $0.7-1.6 \mathrm{~K}$ by $0.3 \mathrm{~K}, 2-4 \mathrm{~K}$ by $0.5 \mathrm{~K}$. In the lower right panel, the contour levels are $0.3-1.2 \mathrm{~K}$ by $0.3 \mathrm{~K}, 1.5-6.5 \mathrm{~K}$ by $0.5 \mathrm{~K}$. 

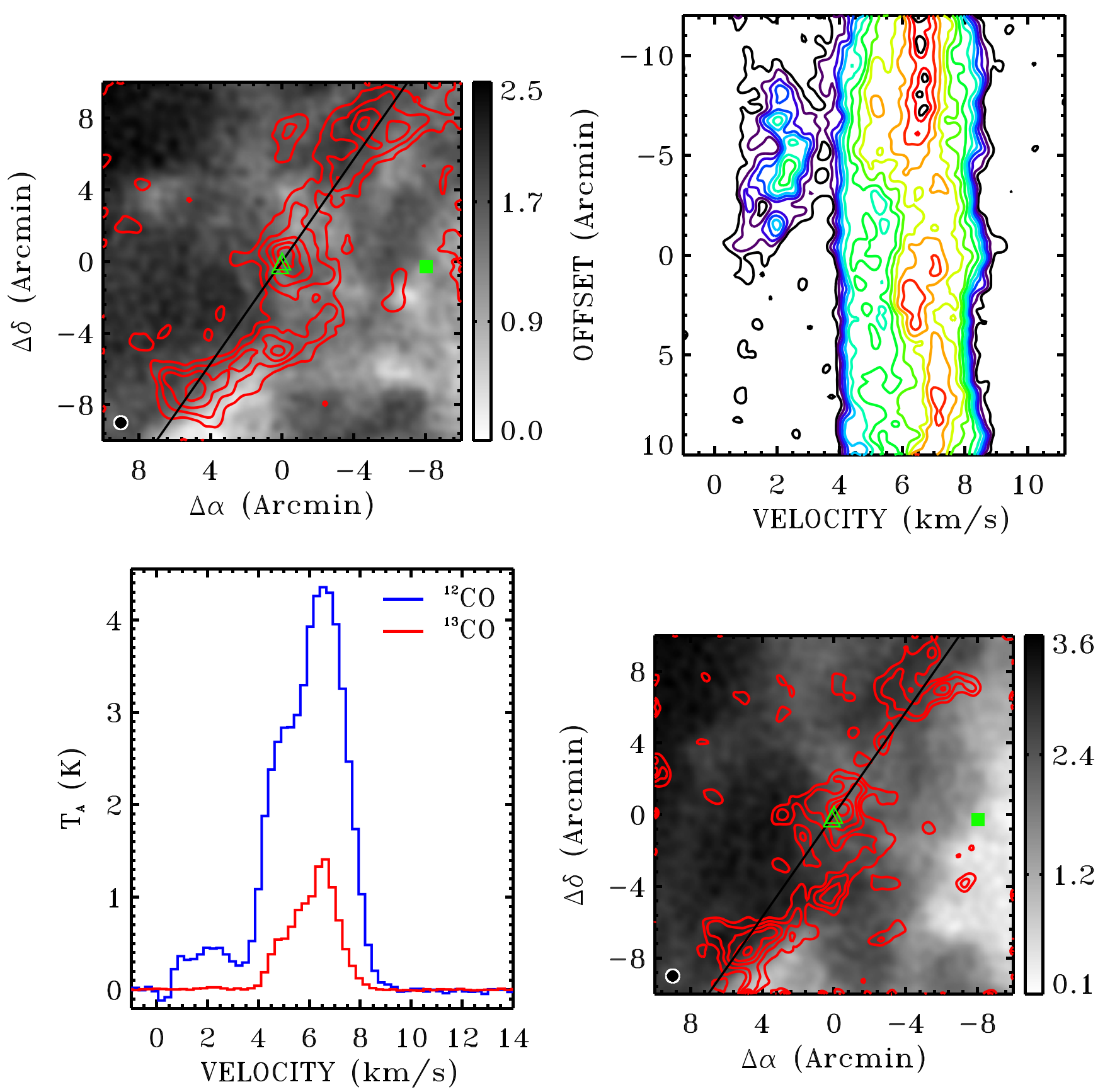

Figure 38. TMO 37 (SST 043334.0+242117). Same as Figure 14 except for the following. In the upper left panel, the integrated intervals for the red lobe and grayscale images are $8-9.5 \mathrm{~km} \mathrm{~s}^{-1}$ and $6-7 \mathrm{~km} \mathrm{~s}^{-1}$, respectively. The red contour levels are $30 \%, 45 \%, \ldots, 90 \%$ of the peak value. In the upper right panel, the position angle is $35^{\circ}$ and the contour levels are $0.4-1.6 \mathrm{~K}$ by $0.3 \mathrm{~K}, 2-5 \mathrm{~K}$ by $0.5 \mathrm{~K}$.

The contour map, P-V diagram, channel maps, and Gaussianfitted curve helped us not only to analyze the morphology, but also to determine the confidence level of a bubble. The bubble candidates were classified into six categories according to the characteristic of the above four types of plots. The criteria for bubble classification, as well as the numbers and ratios of bubbles in different classes are illustrated in Table 6 . In this table, " $x$ " means that it meets a certain condition. For each type of plot, the conditions are as follow.

a. There is an obvious bubble-like structure in the contour map. b. The P-V diagram has an obvious circular or "V" structure.

c. There is an obvious bubble-like structure in the channel map and the radius of the bubble is increasing or decreasing with the channel.

d. The average intensity distribution can be fit with a Gaussian profile.

Meeting all four of the above items is required for a high ranking (Class A). If the plots only meet (b) and (c), then we divided the bubbles into the lower ranking (Class B1) because only the expanding velocity is detected but there is 

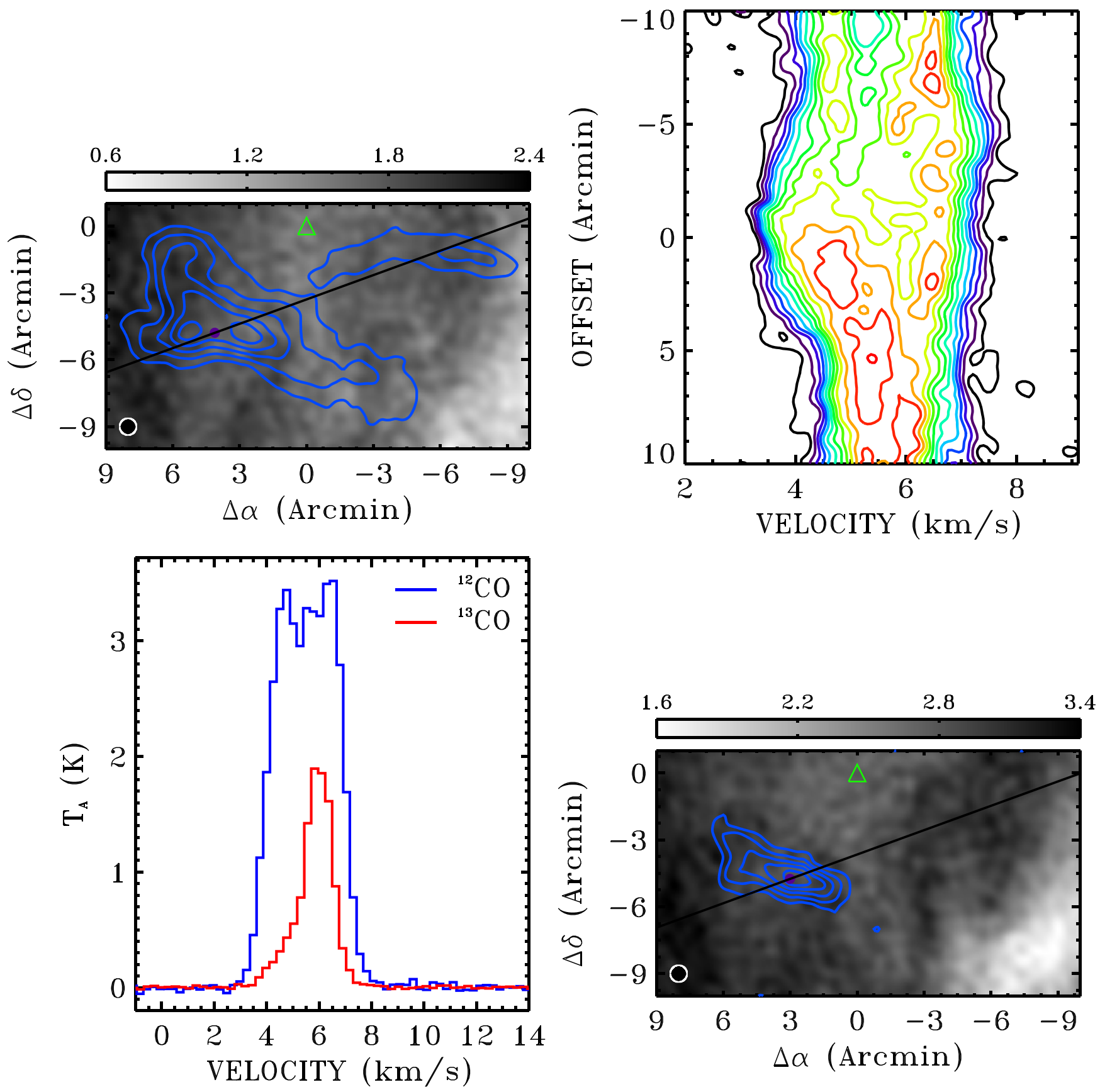

Figure 39. TMO_38 (SST 043336.7+260949). Same as Figure 35 except for the following. In the upper left panel, the integrated intervals for the blue lobe and grayscale image are $3.0-4.1 \mathrm{~km} \mathrm{~s}^{-1}$ and $5.5-6.5 \mathrm{~km} \mathrm{~s}^{-1}$, respectively. The blue contour levels are $30 \%, 45 \%, \ldots, 90 \%$ of the peak value. In the upper right panel, the position angle is $70^{\circ}$.

no obvious bubble-like structure and good Gaussian fitted profile. Then, B2, B3, and B4 are in descending order of ranking. A candidate bubble only meeting (a) is assigned the lowest ranking (Class C) because gas with expanding velocity is not obvious.

Following the above procedures, we found 37 bubbles in the entire $100 \mathrm{deg}^{2}$ area of the Taurus molecular cloud. Each bubble is referred as a "Taurus Molecular Bubble" (TMB). The positions and classifications are listed in Table 7. The number and percentage of each class are illustrated in Table 6.

\subsection{Morphology of Bubbles}

The ${ }^{13} \mathrm{CO}$ integrated intensity maps, $\mathrm{P}-\mathrm{V}$ diagrams, Gaussian fitting profiles, and channel maps for the bubbles are presented in Figures 58-94. If the morphology of a contour map is a closed ring, then we considered it to be an expanding bubble. If the ring on the contour map is incomplete, then we referred to it as a broken bubble. There are 3 expanding bubbles (TMB_07, TMB_10, and TMB_24) and 34 broken bubbles among all of the bubbles in Taurus. 

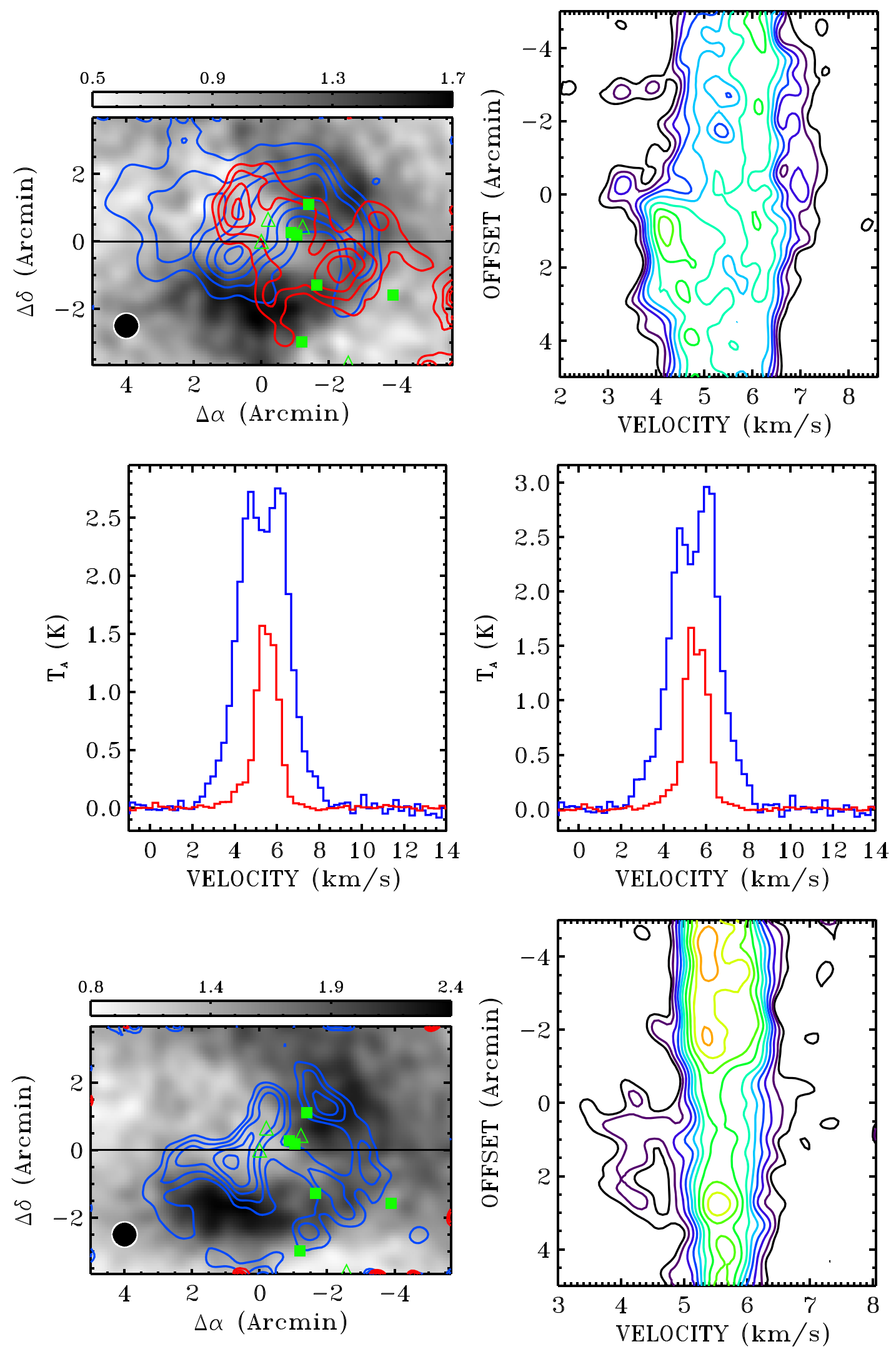

Figure 40. TMO 39 (SST 043557.6+225357). Same as Figure 29 except for the following. In the upper left panel, the integrated intervals for the blue lobe, red lobe, and grayscale images are $2-4 \mathrm{~km} \mathrm{~s}^{-1}, 7-8 \mathrm{~km} \mathrm{~s}^{-1}$, and $5-6 \mathrm{~km} \mathrm{~s}^{-1}$, respectively. The blue and red contour levels are $35 \%, 50 \%, \ldots, 95 \%$ and $60 \%, 70 \%, \ldots, 90 \%$ of their peak value, respectively. In the upper right panel, the contour levels are $0.7-1.6 \mathrm{~K}$ by $0.3 \mathrm{~K}, 2-7 \mathrm{~K}$ by $0.5 \mathrm{~K}$. In the lower right panel, the contour levels are $0.15-0.9 \mathrm{~K}$ by $0.15 \mathrm{~K}, 1.1-1.9 \mathrm{~K}$ by $0.2 \mathrm{~K}$, and $2.2-4 \mathrm{~K}$ by $0.3 \mathrm{~K}$. 

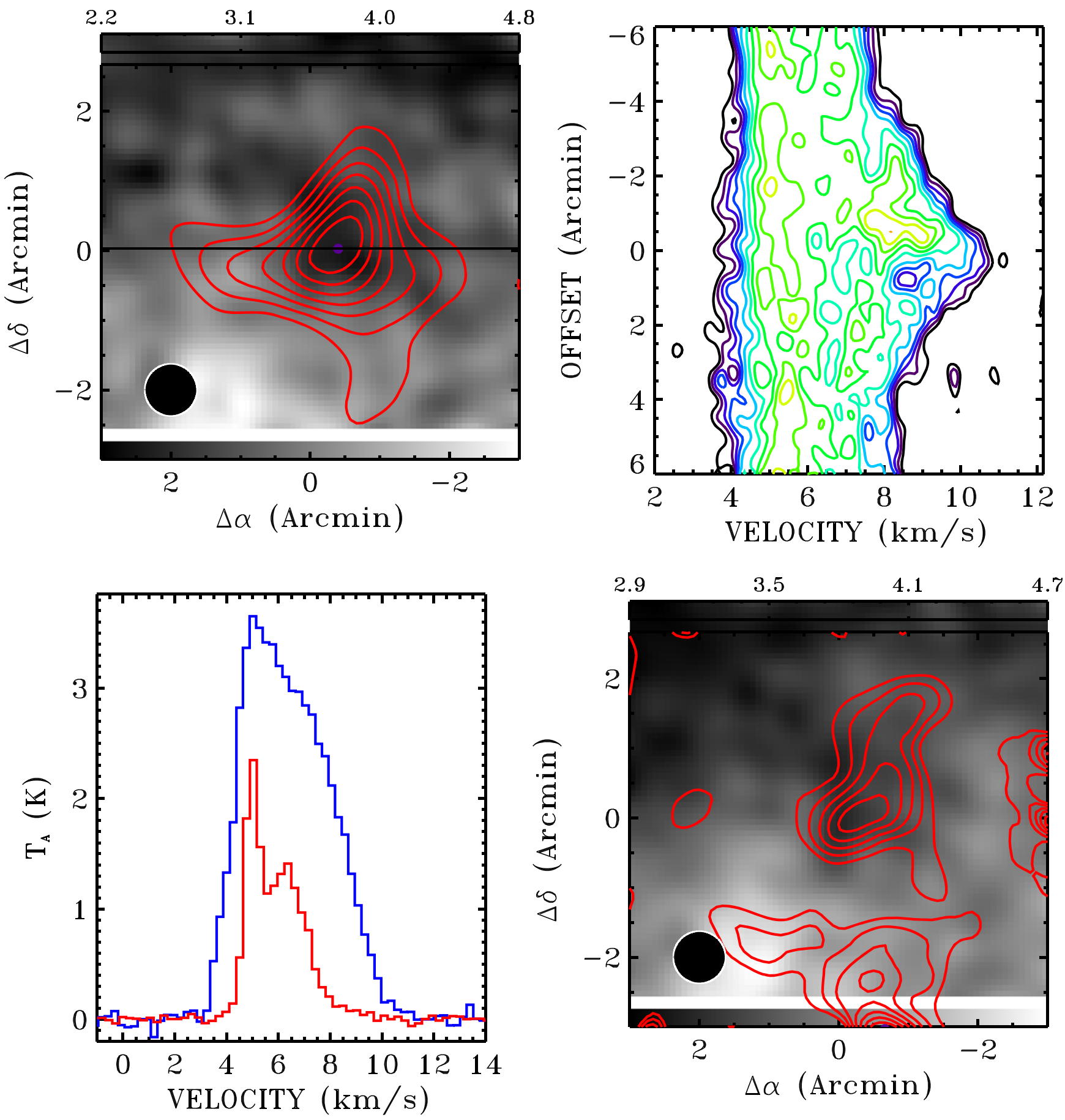

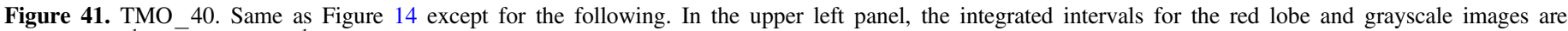

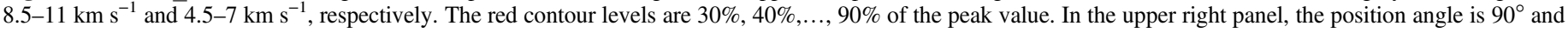
the contour levels are $0.7-1.6 \mathrm{~K}$ by $0.3 \mathrm{~K}, 2-5 \mathrm{~K}$ by $0.5 \mathrm{~K}$.

\subsection{Physical Parameters of Bubbles}

To examine the impact of bubbles on the host cloud, we calculated the mass, momentum, kinetic energy, dynamic timescale, and energy deposition rate of the bubbles. Assuming the ${ }^{13} \mathrm{CO}(1-0)$ emission of the bubble is optically thin, the total column density is derived as follows:

$$
N_{\text {tot }}\left({ }^{13} \mathrm{CO}\right)=\frac{3 k^{2} T_{\mathrm{ex}} f_{\tau}}{4 \pi^{3} \mu_{\mathrm{d}}^{2} h \nu^{2} \exp \left(-h \nu / k T_{\mathrm{ex}}\right)} \int T_{\mathrm{s}} d \nu,
$$



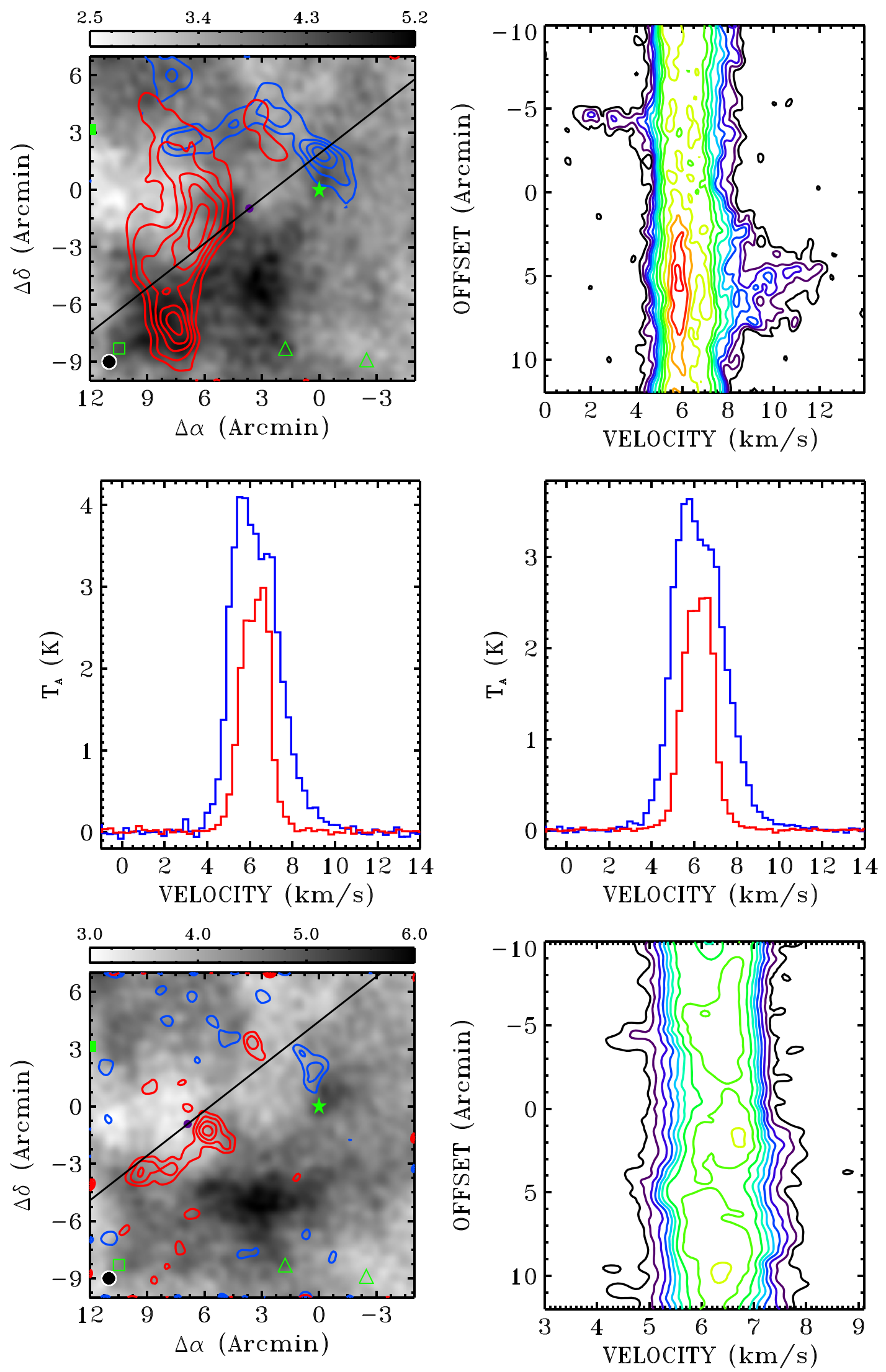

Figure 42. TMO_41 (SST 043913.8+255320). Same as Figure 29 except for the following. In the upper left panel, the integrated intervals for the blue lobe and grayscale images are $1.3-4.5 \mathrm{~km} \mathrm{~s}^{-1}$ and $5-6 \mathrm{~km} \mathrm{~s}^{-1}$, respectively. The blue and red contour levels are $45 \%, 60 \%, \ldots, 90 \%$ and $30 \%, 45 \%, \ldots, 90 \%$ of their peak value, respectively. In the upper right panel, the position angle is $52^{\circ}$ and the contour levels are $0.4-1.6 \mathrm{~K}$ by $0.3 \mathrm{~K}, 2-5 \mathrm{~K}$ by $0.5 \mathrm{~K}$. In the lower left panel, the blue and red contour levels are $60 \%, 75 \%, 90 \%$ and $45 \%, 60 \%, \ldots, 90 \%$ of their peak value, respectively. In the lower right panel, the contour levels are $0.1-1.6 \mathrm{~K}$ by $0.3 \mathrm{~K}, 2-5 \mathrm{~K}$ by $0.5 \mathrm{~K}$. 

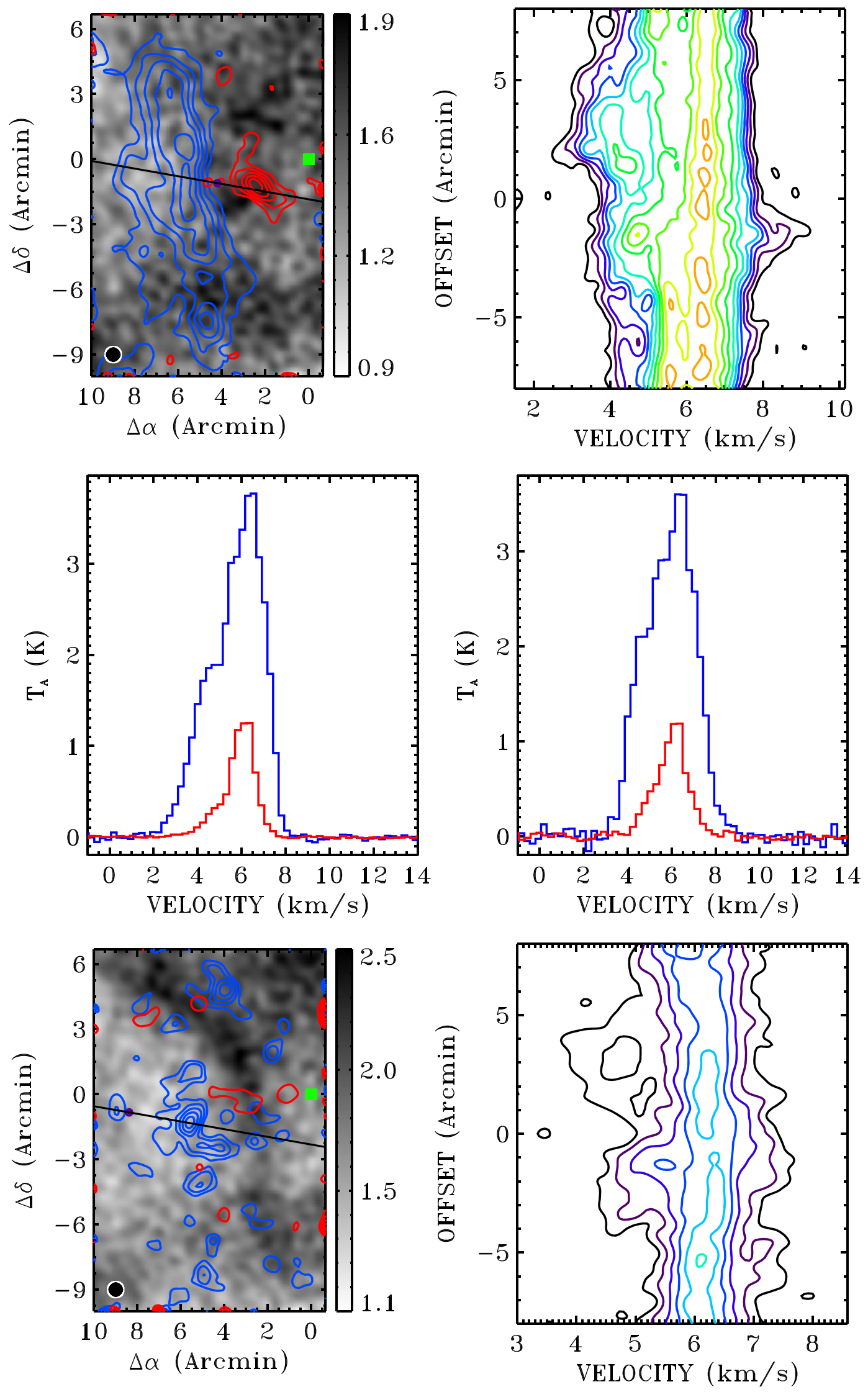

Figure 43. TMO 42 (SST 044802.3+253359). Same as Figure 29 except for the following. In the upper left panel, the integrated intervals for the blue lobe, red lobe, and grayscale image are $2.8-3.5 \mathrm{~km} \mathrm{~s}^{-1}, 8-9.5 \mathrm{~km} \mathrm{~s}^{-1}$, and $5-7 \mathrm{~km} \mathrm{~s}^{-1}$, respectively. The blue and red contour levels are both $30 \%, 45 \%, \ldots, 90 \%$ of their peak value. In the upper right panel, the position angle is $100^{\circ}$ and the contour levels are $0.4-1.6 \mathrm{~K}$ by $0.3 \mathrm{~K}, 2-5 \mathrm{~K}$ by $0.5 \mathrm{~K}$. In the lower right panel, the contour levels are $0.1-1.6 \mathrm{~K}$ by $0.3 \mathrm{~K}, 2-6.5 \mathrm{~K}$ by $0.5 \mathrm{~K}$. 

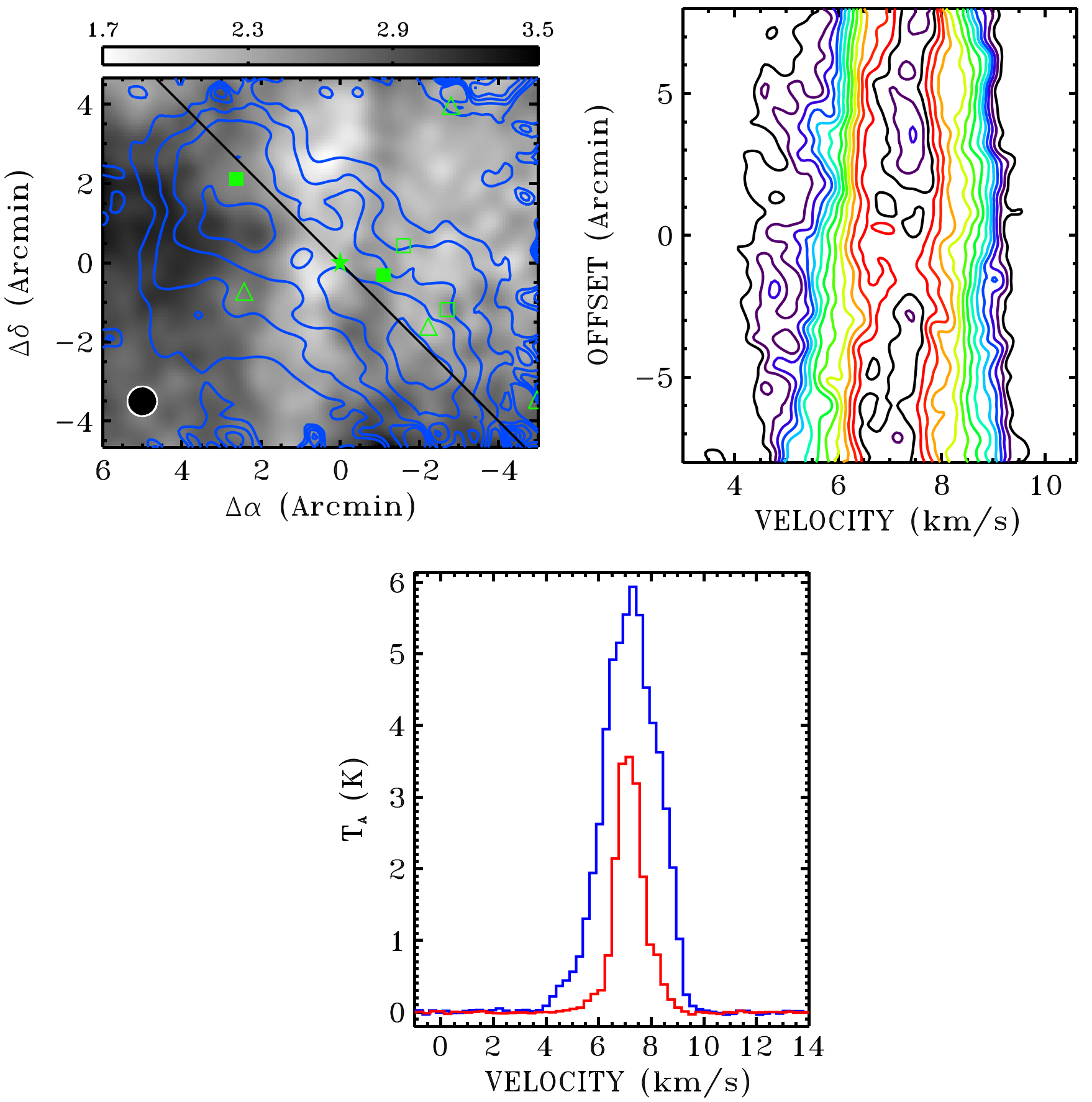

Figure 44. TMO_43 (SST 041851.4+282026). Same as Figure 6 except for the following. In the upper left panel, the integrated intervals for the blue lobe and grayscale images are $4-5 \mathrm{~km} \mathrm{~s}^{-1}$ and $6.5-8 \mathrm{~km} \mathrm{~s}^{-1}$, respectively. The blue contour levels are $40 \%, 50 \%, \ldots, 90 \%$ of the peak value. In the upper right panel, the position angle is $135^{\circ}$ and the contour levels are $0.4-1.6 \mathrm{~K}$ by $0.3 \mathrm{~K}, 2-6.5 \mathrm{~K}$ by $0.5 \mathrm{~K}$.

where $k=1.38 \times 10^{-16} \mathrm{erg} \mathrm{\textrm {K } ^ { - 1 }}, \quad h=6.626 \times 10^{-27} \mathrm{erg} \mathrm{s}$, $\mu_{\mathrm{d}}=0.112 \times 10^{-18} \mathrm{esu}, \quad$ and $\quad \nu=110.2014 \times 10^{9} \mathrm{~Hz}$. The excitation temperature, $T_{\mathrm{ex}}$, is assumed to be $25 \mathrm{~K}$. $T_{\mathrm{s}}$ is the observed source antenna temperature with proper correction for antenna efficiency. The optical depth correction factor, $f_{\tau}$, is estimated from the following formulae (Qian et al. 2012):

$$
f_{\tau}=\frac{\int \tau\left({ }^{13} \mathrm{CO}\right) d v}{\int\left[1-e^{-\tau\left({ }^{13} \mathrm{CO}\right)}\right] d v},
$$

where $\tau\left({ }^{13} \mathrm{CO}\right)$ is the opacity of the ${ }^{13} \mathrm{CO}$ transition. Assuming equal excitation temperatures for ${ }^{13} \mathrm{CO}$ and ${ }^{12} \mathrm{CO}$, we can obtain

$$
\frac{T\left({ }^{12} \mathrm{CO}\right)}{T\left({ }^{13} \mathrm{CO}\right)}=\frac{1-e^{-\tau\left({ }^{12} \mathrm{CO}\right)}}{1-e^{-\tau\left({ }^{13} \mathrm{CO}\right)}},
$$

where $T\left({ }^{12} \mathrm{CO}\right)$ and $T\left({ }^{13} \mathrm{CO}\right)$ are the brightness temperatures of ${ }^{12} \mathrm{CO}$ and ${ }^{13} \mathrm{CO}$, respectively. $\tau\left({ }^{12} \mathrm{CO}\right)$ is the opacity of the ${ }^{12} \mathrm{CO}$ transition. Assuming the ${ }^{12} \mathrm{CO}$ emission from the bubbles is optically thick $\left(\tau\left({ }^{12} \mathrm{CO}\right) \gg 1\right)$, the opacity of ${ }^{13} \mathrm{CO}$ can be 

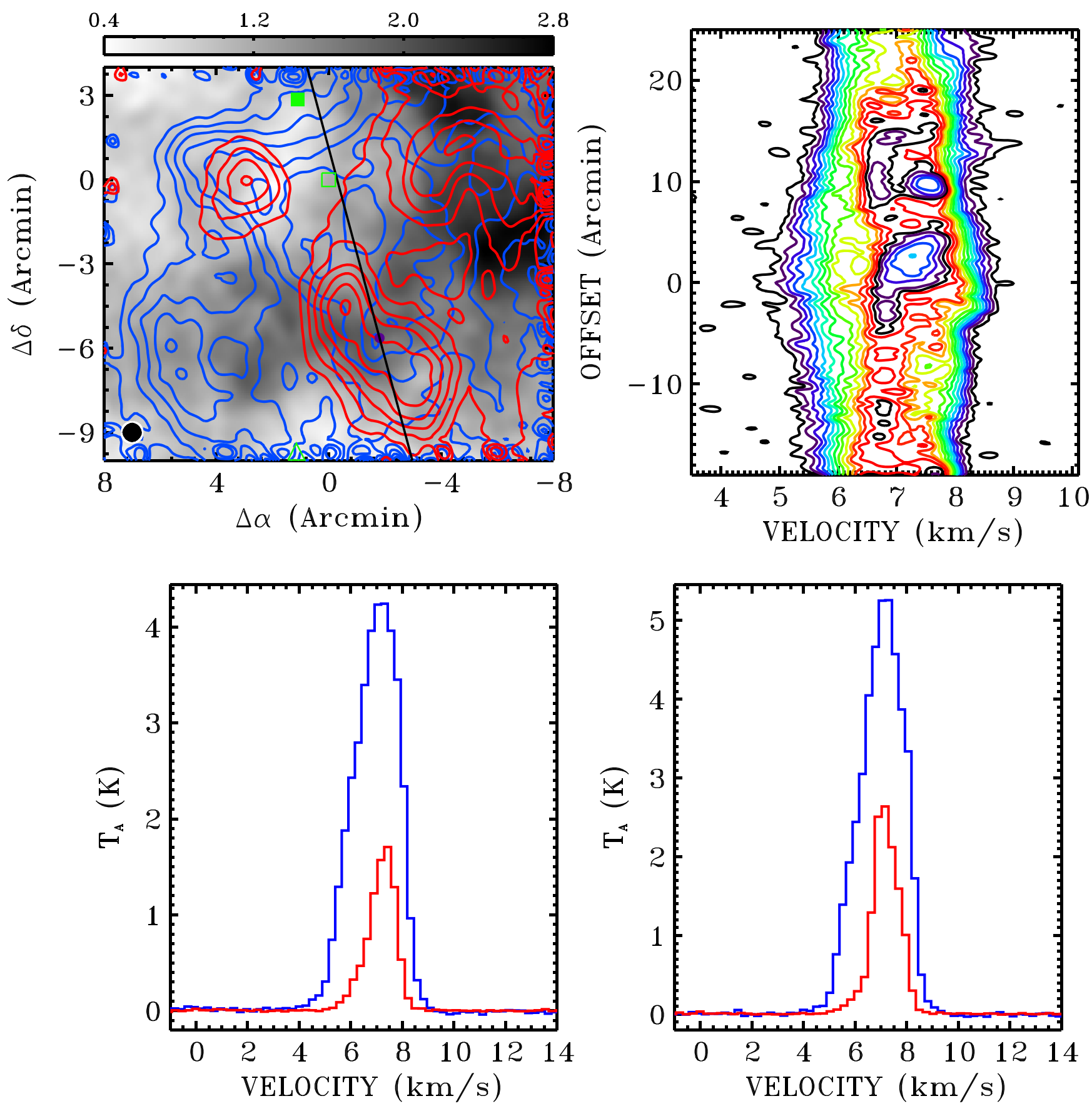

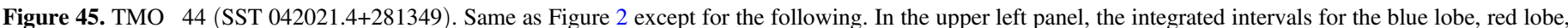

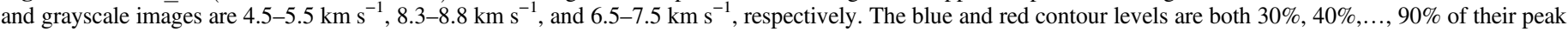
value. In the upper right panel, the position angle is $165^{\circ}$ and the contour levels are $0.4-5.5 \mathrm{~K}$ by $0.3 \mathrm{~K}$.

obtained from

$$
\tau\left({ }^{13} \mathrm{CO}\right)=-\ln \left(1-\frac{T\left({ }^{13} \mathrm{CO}\right)}{T\left({ }^{12} \mathrm{CO}\right)}\right) .
$$

Using the column density and area, we can obtain the bubble mass. Using the bubble mass and expansion velocity, we can then obtain the momentum and kinetic energy of the bubble using $\quad P_{\text {bubble }}=M_{\text {bubble }} V_{\exp } \quad$ and $\quad E_{\text {bubble }}=\frac{1}{2} M_{\text {bubble }} V_{\exp }^{2}$, respectively. The kinetic timescale of the bubble can be calculated as $t_{\text {kinetic }}=R / V_{\text {exp }}$, where $R$ is the radius and $V_{\exp }$ is the expansion velocity of the bubble. The bubble energy injection rate, $L_{\text {bubble, }}$ can be estimated as $L_{\text {bubble }}=E_{\text {bubble }} / t_{\text {kinetic }}$.

The physical parameters of all bubbles are listed in Table 7. The momentum and kinetic energy are lower limits mainly because of the underestimate of the minimum expansion velocity. The total mass, momentum, energy, and energy 

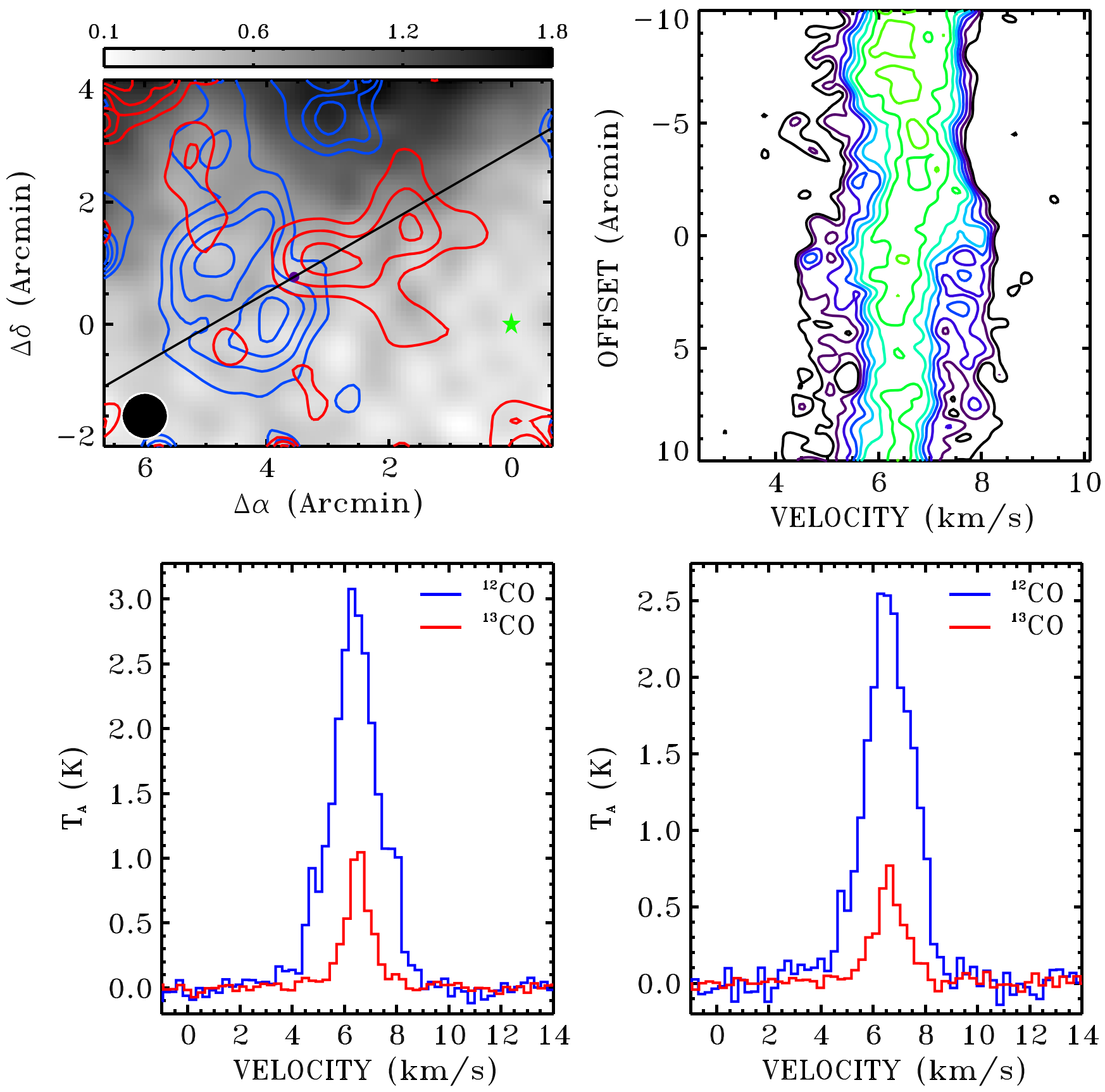

Figure 46. TMO 45 (SST 042653.3+255858). Same as Figure 2 except for the following. In the upper left panel, the integrated intervals for the blue lobe, red lobe, and gray-scale image are $2-5.5 \mathrm{~km} \mathrm{~s}^{-1}, 7.5-10 \mathrm{~km} \mathrm{~s}^{-1}$, and $6-7 \mathrm{~km} \mathrm{~s}^{-1}$, respectively. The blue and red contour levels are both $60 \%, 70 \%, \ldots, 90 \%$ of their peak value. In the upper right panel, the position angle is $60^{\circ}$ and the contour levels are $0.4-1.6 \mathrm{~K}$ by $0.3 \mathrm{~K}, 2-3.5 \mathrm{~K}$ by $0.5 \mathrm{~K}$.

injection rate of all of the detected bubbles in the Taurus molecular cloud are about $1704 M_{\odot}, 3780 M_{\odot} \mathrm{km} \mathrm{s}^{-1}$, $9.2 \times 10^{46} \mathrm{erg}$, and $6.4 \times 10^{33} \mathrm{erg} \mathrm{s}^{-1}$, respectively.

The distribution of radius, mass, energy, and dynamical timescale of bubbles are shown in Figure 95. The radius of bubbles is in the range $0.28-1.9 \mathrm{pc}$. $78 \%$ of the bubbles are smaller than $1 \mathrm{pc}$. The mass of $65 \%$ of the bubbles is between $5 M_{\odot}$ and $50 M_{\odot}$. The bubbles with mass lower than $5 M_{\odot}$ and higher than $50 M_{\odot}$ account for $16 \%$ and $19 \%$, respectively. The highest bubble mass is $386 M_{\odot}$. The energy of $60 \%$ of bubbles is in the range of $10^{44}-10^{45} \mathrm{erg}$. The bubbles with energy lower than $10^{44} \mathrm{erg}$ and higher than $10^{45} \mathrm{erg}$ account for $16 \%$ and $24 \%$, respectively. The dynamical timescales of bubbles are between $10^{5}$ and $10^{6}$ years. Almost $95 \%$ of bubbles are younger than $10^{6}$ years. Compared with the outflows, the bubbles have about 110 times greater mass and 24 times higher energy. The extents of the bubbles are larger than the outflows, as can be seen from Figure 96. The dynamical timescales of bubbles are longer than those of outflows. 

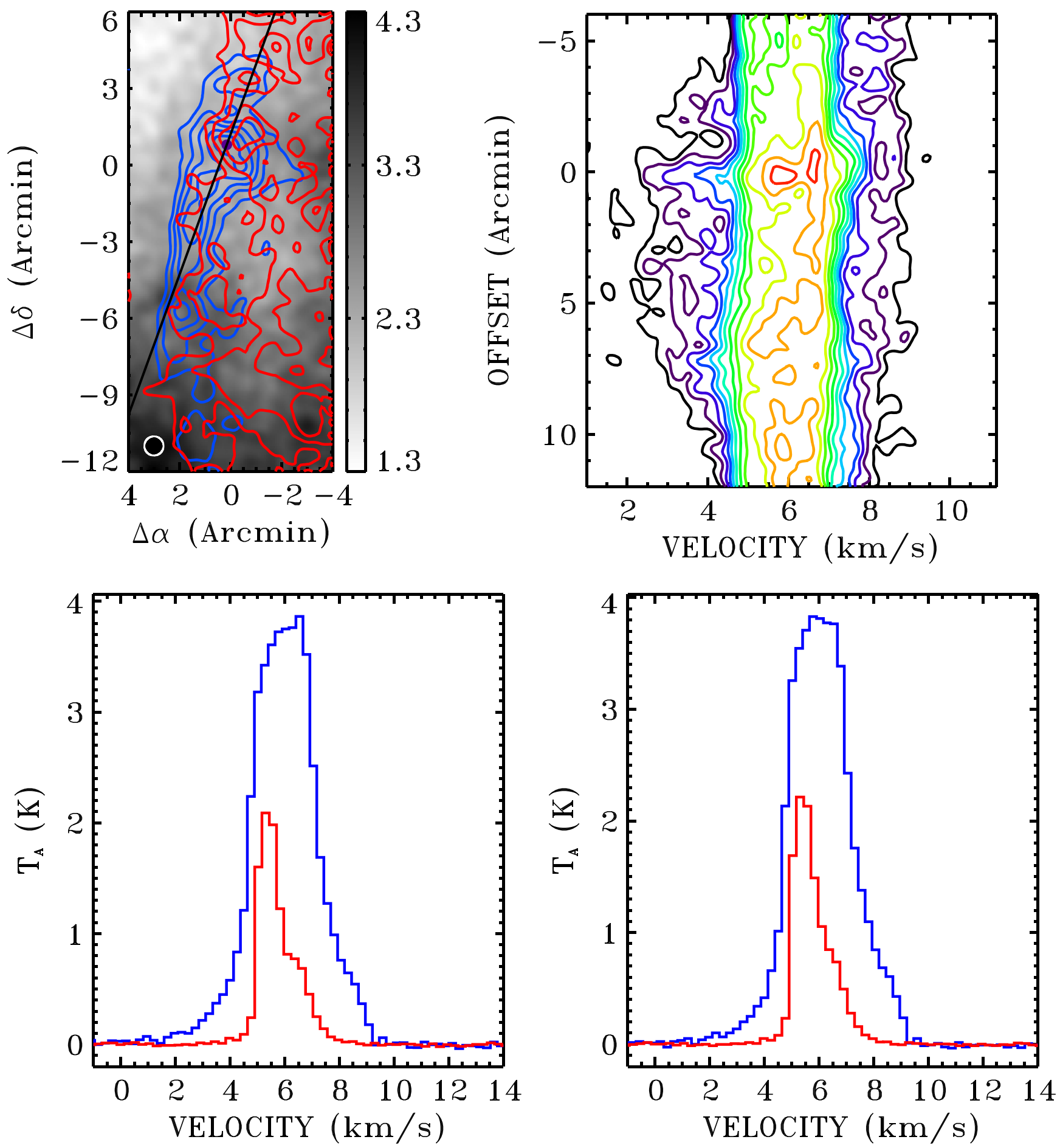

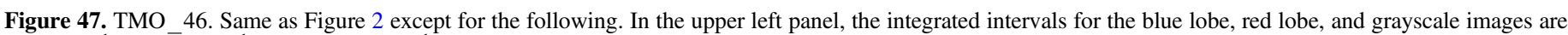

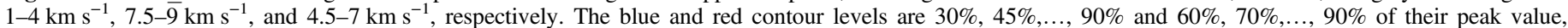
respectively. In the upper right panel, the position angle is $20^{\circ}$ and the contour levels are $0.4-1.6 \mathrm{~K}$ by $0.3 \mathrm{~K}, 2-4.5 \mathrm{~K}$ by $0.5 \mathrm{~K}$.

\section{ANALYSIS AND DISCUSSION}

\subsection{Polarity of Outflows in Taurus}

Among the 55 outflows, we found that bipolar, monopolar redshifted, and monopolar blueshifted outflows account for $45 \%, 44 \%$, and $11 \%$, respectively. There are more red lobes than blue ones, which can be seen from the histograms in Figure 57. The occurrence of more red lobes may result from the fact that Taurus is thin (Qian et al. 2014). Red lobes tend to be smaller and younger. The total mass and energy of red lobes is similar to blue lobes on average, which can be seen from the upper right panel and lower left panels of Figure 57. 

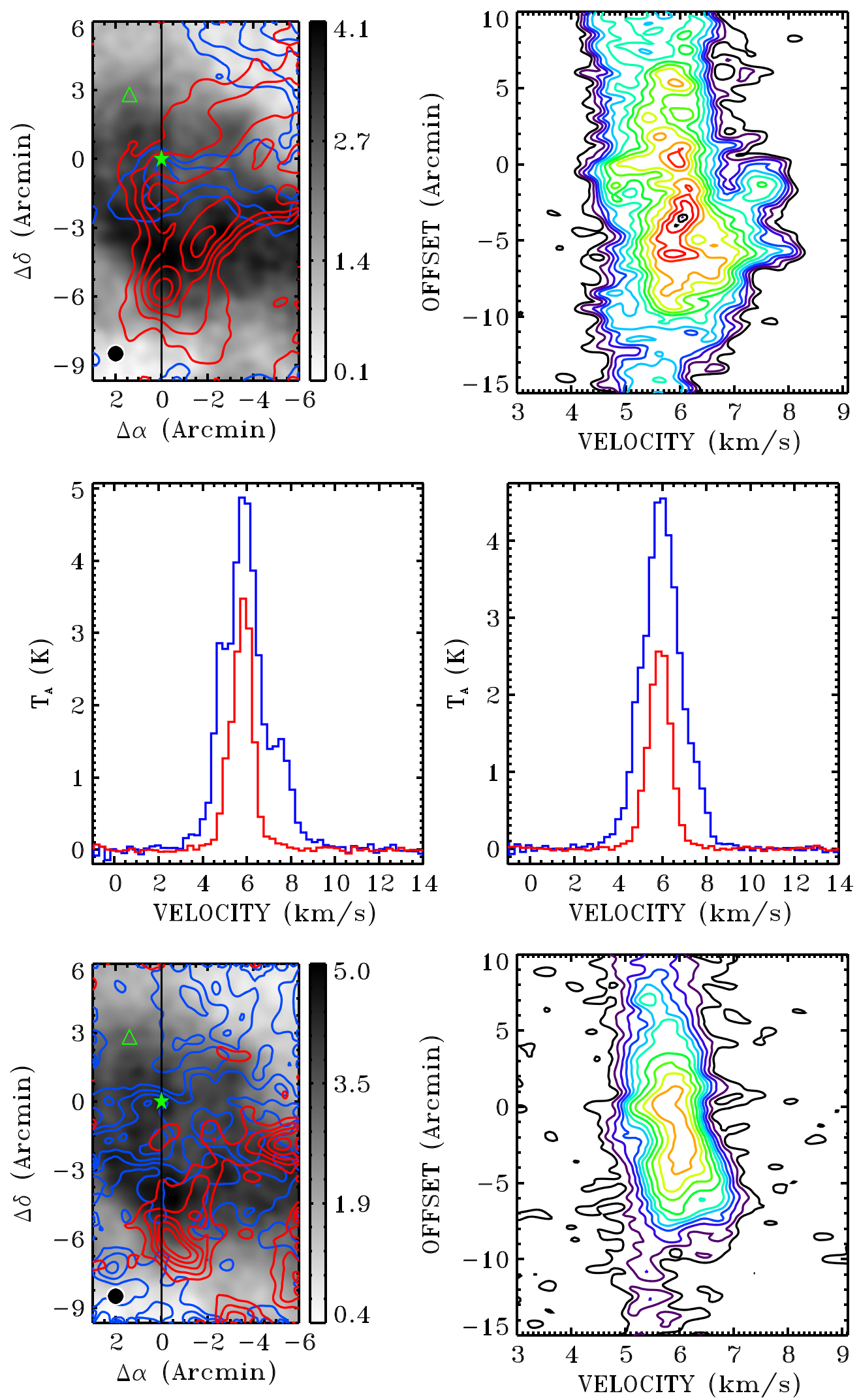

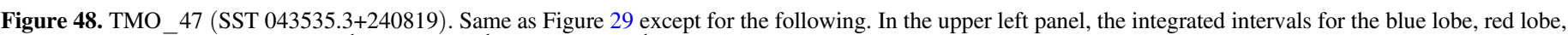

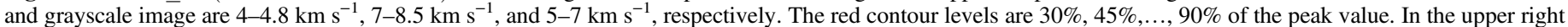

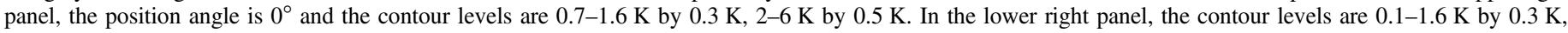
$2-4 \mathrm{~K}$ by $0.5 \mathrm{~K}$. 

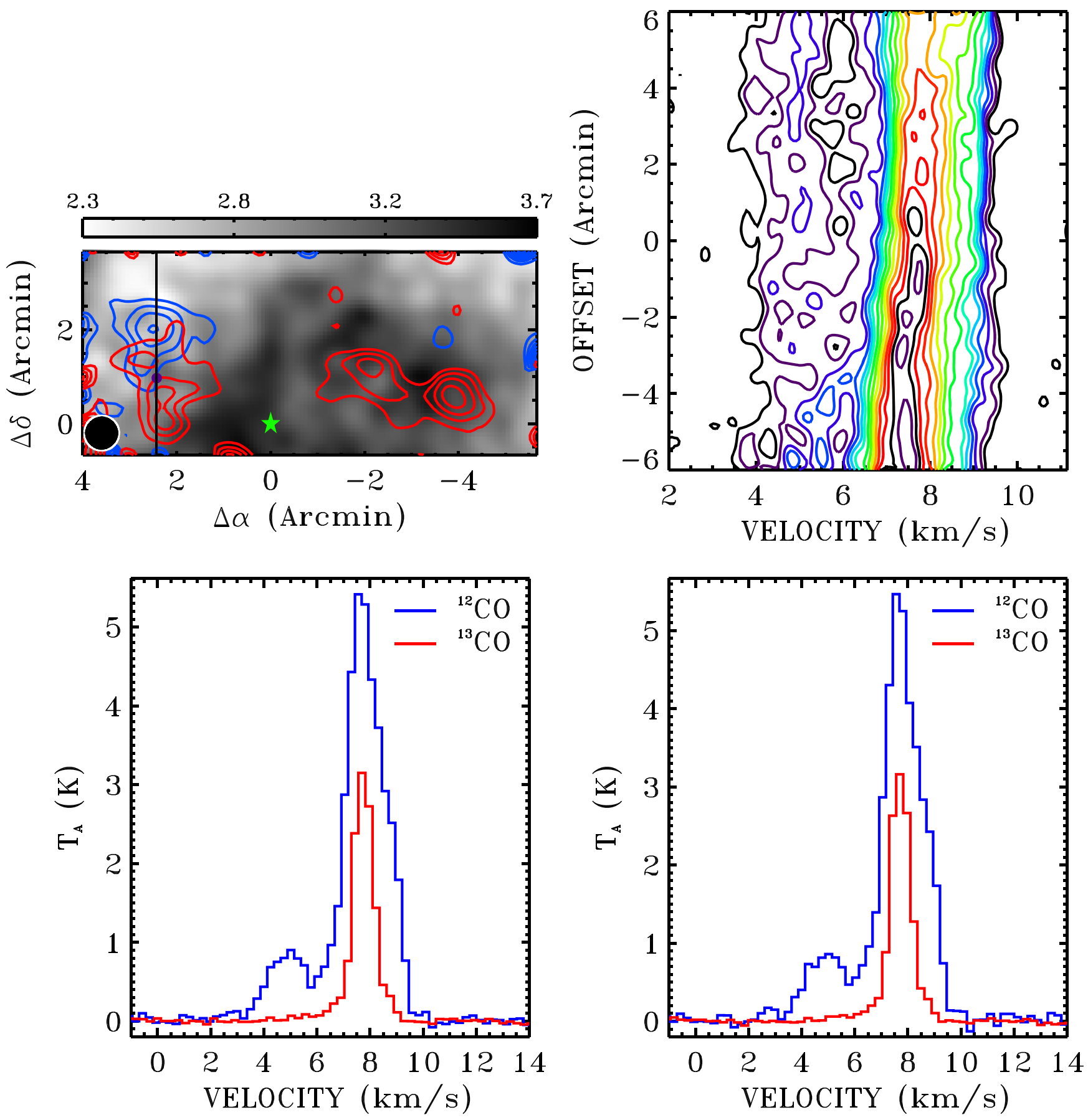

Figure 49. TMO_48 (SST 041535.6+284741). Same as Figure 2 except for the following. In the upper left panel, the integrated intervals for the blue lobe, red lobe, and grayscale image are -1 to $4 \mathrm{~km} \mathrm{~s}^{-1}, 9.25-13 \mathrm{~km} \mathrm{~s}^{-1}$, and $6.5-8.5 \mathrm{~km} \mathrm{~s}^{-1}$, respectively. The red contour levels are $40 \%, 50 \%, \ldots, 90 \%$ of the peak value. In the upper right panel, the position angle is $0^{\circ}$ and contour levels are $0.4-1.6 \mathrm{~K}$ by $0.3 \mathrm{~K}, 2-6.5 \mathrm{~K}$ by $0.5 \mathrm{~K}$.

\subsection{The Driving Sources of Outflows and Bubbles in Taurus}

The outflows are driven by four types of YSOs. From Table 4, we can see that Class I, Flat, Class II, and Class III account for $36.4 \%, 18.2 \%, 27.3 \%$, and $12.7 \%$ of all the driving sources, respectively. Figure 96 shows the distribution of different classes of YSOs driving outflows (hereafter outflow-driving YSOs) and YSOs inside the bubbles (hereafter bubble-driving YSOs). The rough dividing line shows that there are more outflow-driving
YSOs in Class I, Flat, and Class II and few outflow-driving YSOs in Class III, which indicates that outflows are more likely to appear in the earlier stage (Class I) than in the later phase (Class III) of star formation. There are more bubble-driving YSOs of Class II and Class III while there are few bubble-driving YSOs of Class I and Flat, implying that the bubble structures are more likely to occur in the later stage of star formation. From the size of the symbols, we can see that the larger the outflows and bubbles are, the higher energy they have. 

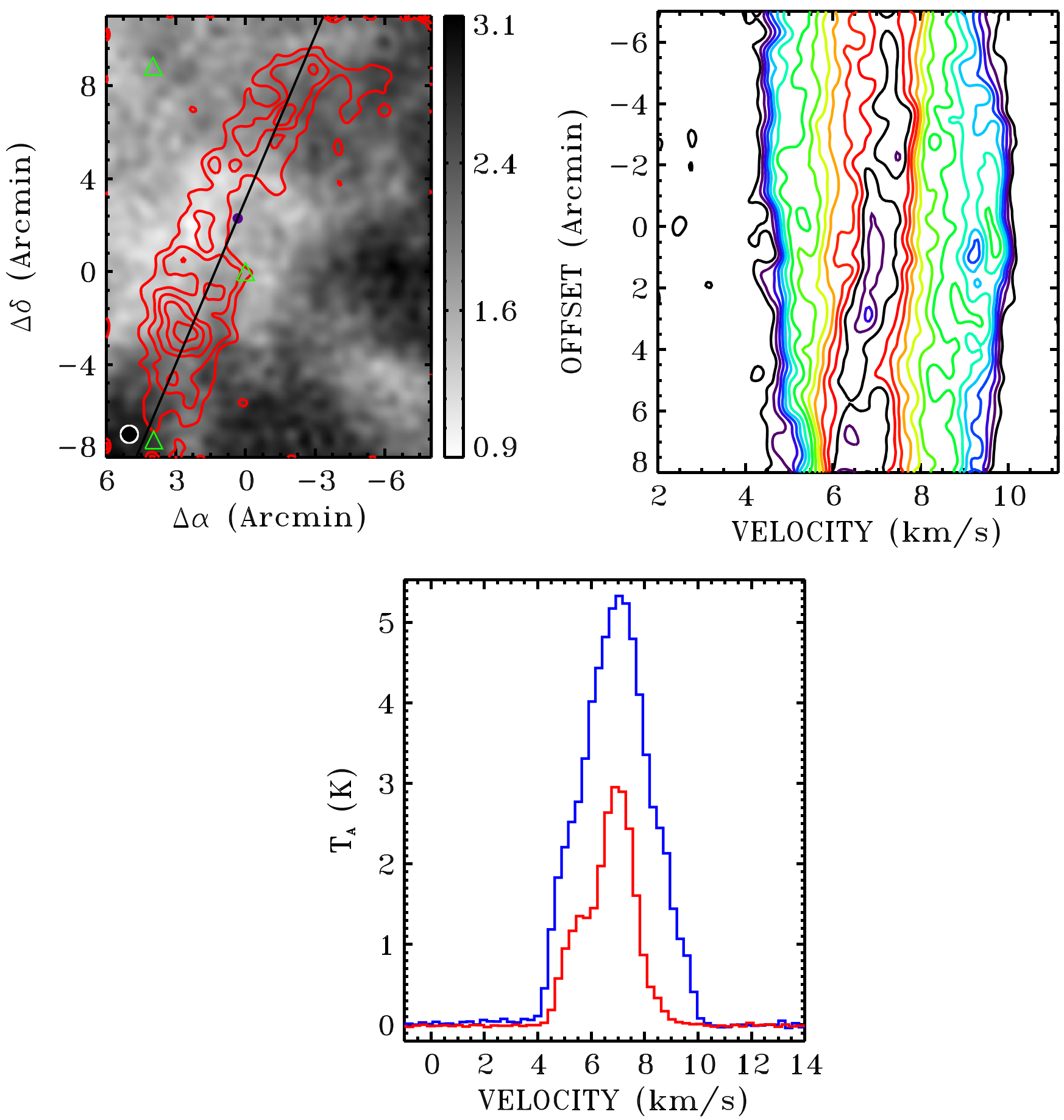

Figure 50. TMO_49 (SST 041733.7+282046). Same as Figure 3 except for the following. In the upper left panel, the integrated interval for the red lobe is $9.5-13 \mathrm{~km} \mathrm{~s}^{-1}$. In the upper right panel, the position angle is $23^{\circ}$ and contour levels are $0.4-1.6 \mathrm{~K}$ by $0.3 \mathrm{~K}, 2-7 \mathrm{~K}$ by $0.5 \mathrm{~K}$.

\subsection{The Feedback of Outflows and Bubbles in Taurus}

Using the complete sample of outflows and bubbles, we can estimate the overall impact of dynamical structures on the Taurus molecular cloud. We investigated whether the outflows and bubbles have enough energy to potentially unbind the entire Taurus molecular cloud or drive the turbulence in the cloud.

\subsubsection{The Energy of Outflows and Bubbles Cannot Balance the Gravitational Binding Energy of the Entire Cloud}

Using a total mass of $1.5 \times 10^{4} M_{\odot}$ (Pineda et al. 2010) and an effective radius of $13.8 \mathrm{pc}$ (Narayanan et al. 2012) for the $100 \mathrm{deg}^{2}$ region of Taurus, we calculated the magnitude of the gravitational binding energy $G M_{\text {cloud }}^{2} / R_{\text {cloud }}$ to be $\sim 1.5 \times 10^{48}$ erg. The total kinetic energy of outflows from the $44 \mathrm{deg}^{2}$ region 

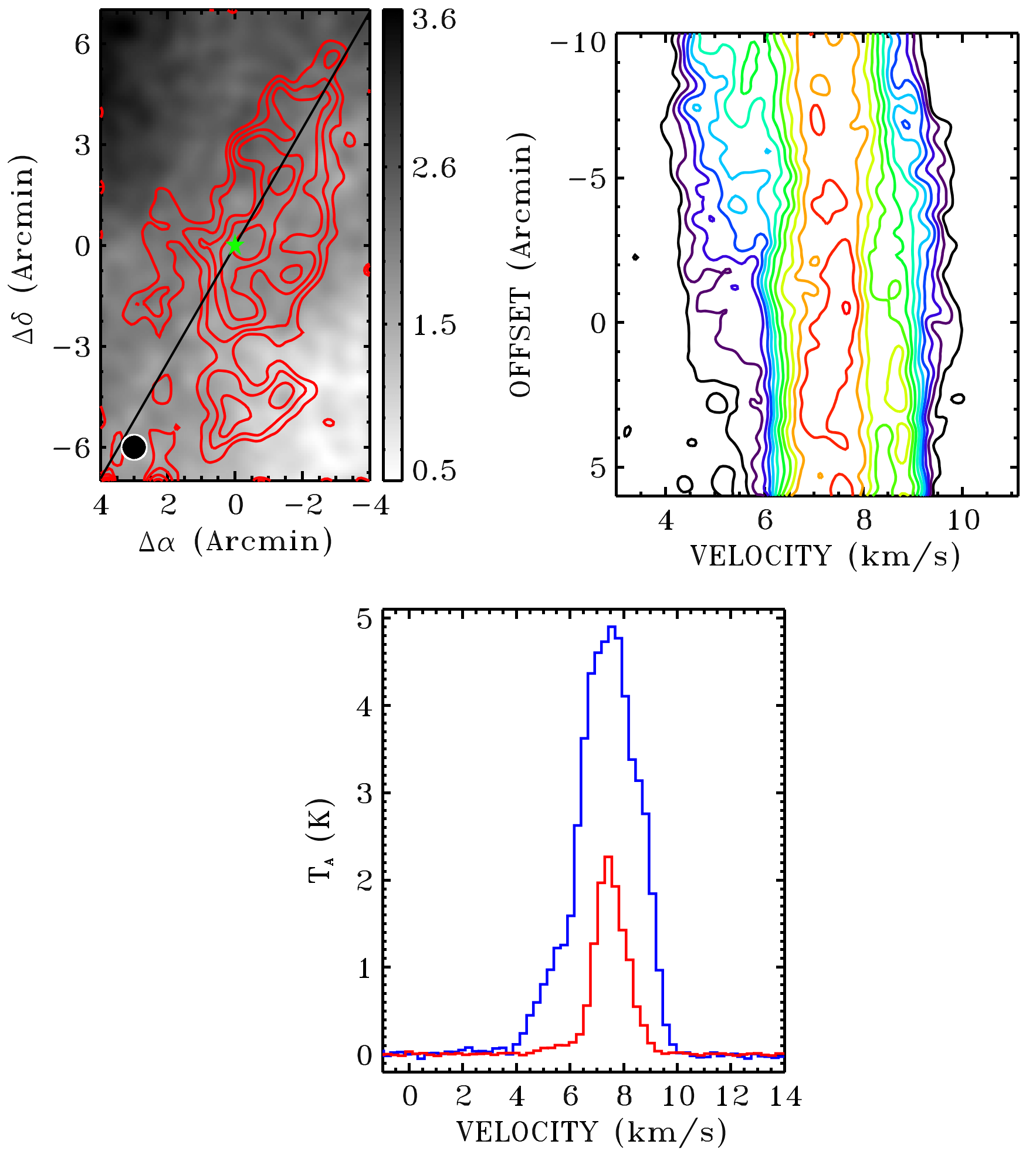

Figure 51. TMO 50 (SST 041810.5+284447). Same as Figure 3 except for the following. In the upper left panel, the integrated intervals for the red lobe and grayscale images are $9.3-10 \mathrm{~km} \mathrm{~s}^{-1}$ and $6.5-8.5 \mathrm{~km} \mathrm{~s}^{-1}$, respectively. The red contour levels are $50 \%, 60 \%, \ldots, 90 \%$ of the peak value. In the upper right panel, the position angle is $30^{\circ}$ and the contour levels are $0.4-1.6 \mathrm{~K}$ by $0.3 \mathrm{~K}, 2-5 \mathrm{~K}$ by $0.5 \mathrm{~K}$.

of Taurus is $3.9 \times 10^{45} \mathrm{erg}$, which is much less than the gravitational binding energy. Given that we searched for outflows around YSOs only in the Spitzer $44 \mathrm{deg}^{2}$ survey region, not the overall area of Taurus, we may well have missed some outflows.
Most of the gas of Taurus is centered on the Spitzer $44 \mathrm{deg}^{2}$ survey region, which can be seen from Figure 2 of Goldsmith et al. (2008). There are not many YSOs outside the Spitzer coverage in Taurus and those YSOs are generally clustered, 

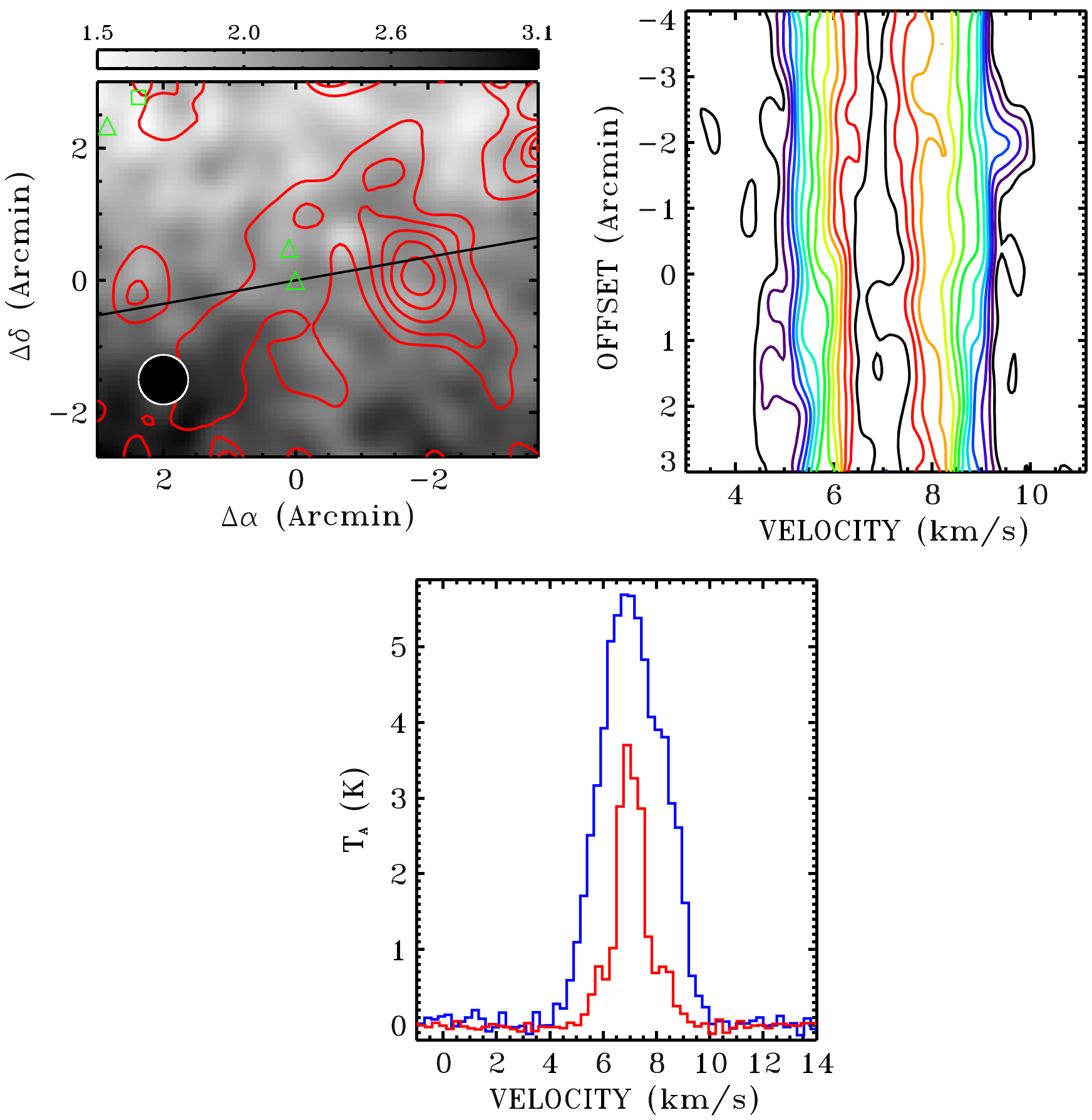

Figure 52. TMO 51 (SST 041831.1+281629). Same as Figure 3 except for the following. In the upper left panel, the integrated intervals for the red lobe and grayscale images are $9.3-10.2 \mathrm{~km} \mathrm{~s}^{-1}$ and $6.5-8 \mathrm{~km} \mathrm{~s}^{-1}$, respectively. The red contour levels are $30 \%, 40 \%, \ldots, 90 \%$ of the peak value. In the upper right panel, the position angle is $80^{\circ}$ and the contour levels are $0.5-2 \mathrm{~K}$ by $0.3 \mathrm{~K}, 2.5-6 \mathrm{~K}$ by $0.5 \mathrm{~K}$.

which can be seen from Figure 1 of Rebull et al. (2011). Therefore, there should be few outflows outside the Spitzer coverage in Taurus and the outflows we found around YSOs in the $44 \mathrm{deg}^{2}$ area account for the majority of outflows in Taurus. Similarly, the total kinetic energy of the detected bubbles in the $100 \mathrm{deg}^{2}$ Taurus region is $9.2 \times 10^{46} \mathrm{erg}$, which also cannot balance the gravitational potential energy of the entire cloud.

\subsubsection{Turbulent Energy is Greater than the Energy of Outflows and Bubbles}

The turbulent energy of the Taurus molecular cloud is given approximately by

$$
E_{\text {turb }}=\frac{1}{2} M_{\text {cloud }} \sigma_{3 \mathrm{~d}}^{2}
$$



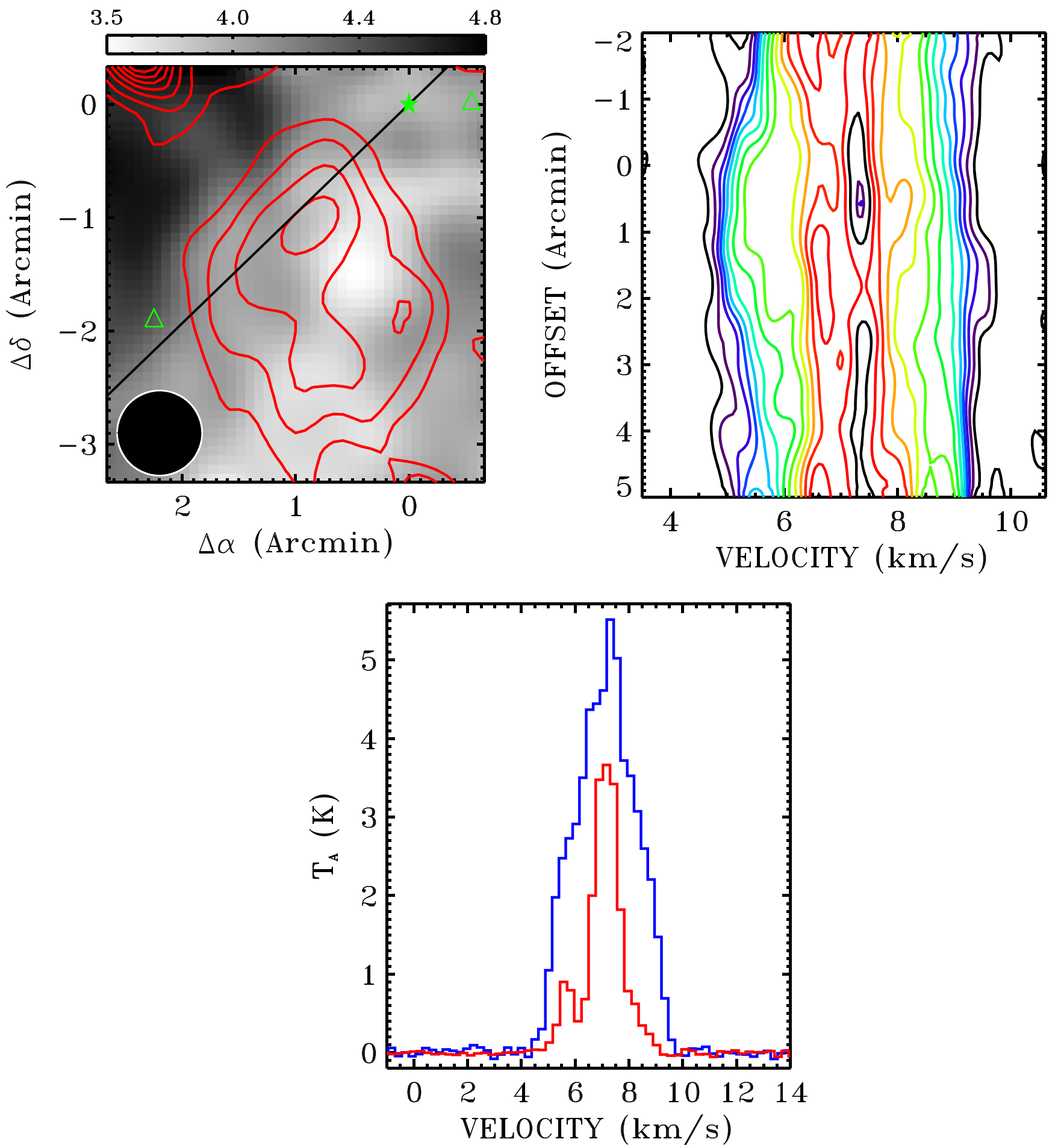

Figure 53. TMO_52 (SST 041831.2+282617). Same as Figure 3 except for the following. In the upper left panel, the integrated intervals for the red lobe and grayscale images are $9.3-9.8 \mathrm{~km} \mathrm{~s}^{-1}$ and $6-8 \mathrm{~km} \mathrm{~s}^{-1}$, respectively. The red contour levels are $30 \%, 40 \%, \ldots, 90 \%$ of the peak value. In the upper right panel, the position angle is $60^{\circ}$ and the contour levels are $0.4-1.6 \mathrm{~K}$ by $0.3 \mathrm{~K}, 2-6 \mathrm{~K}$ by $0.5 \mathrm{~K}$.

where $\sigma_{3 \mathrm{~d}}$ is the three-dimensional turbulent velocity dispersion, which can be calculated by

$$
\sigma_{3 \mathrm{~d}}=\frac{\sqrt{3}}{2 \sqrt{2 \ln 2}} \Delta v_{\mathrm{FWHM}} .
$$

Here, $\Delta v_{\mathrm{FWHM}}=2 \mathrm{~km} \mathrm{~s}^{-1}$ is the one-dimensional FWHM velocity dispersion based on typical ${ }^{13} \mathrm{CO}$ spectra in Taurus (Narayanan et al. 2012). Then, we obtain $\sigma_{3 \mathrm{~d}}=1.47 \mathrm{~km} \mathrm{~s}^{-1}$. The total mass of the $100 \mathrm{deg}^{2}$ region of Taurus is $M_{\text {cloud }}=1.5 \times 10^{4} M_{\odot} \quad($ Pineda $\quad$ et $\quad$ al. 2010). Using 

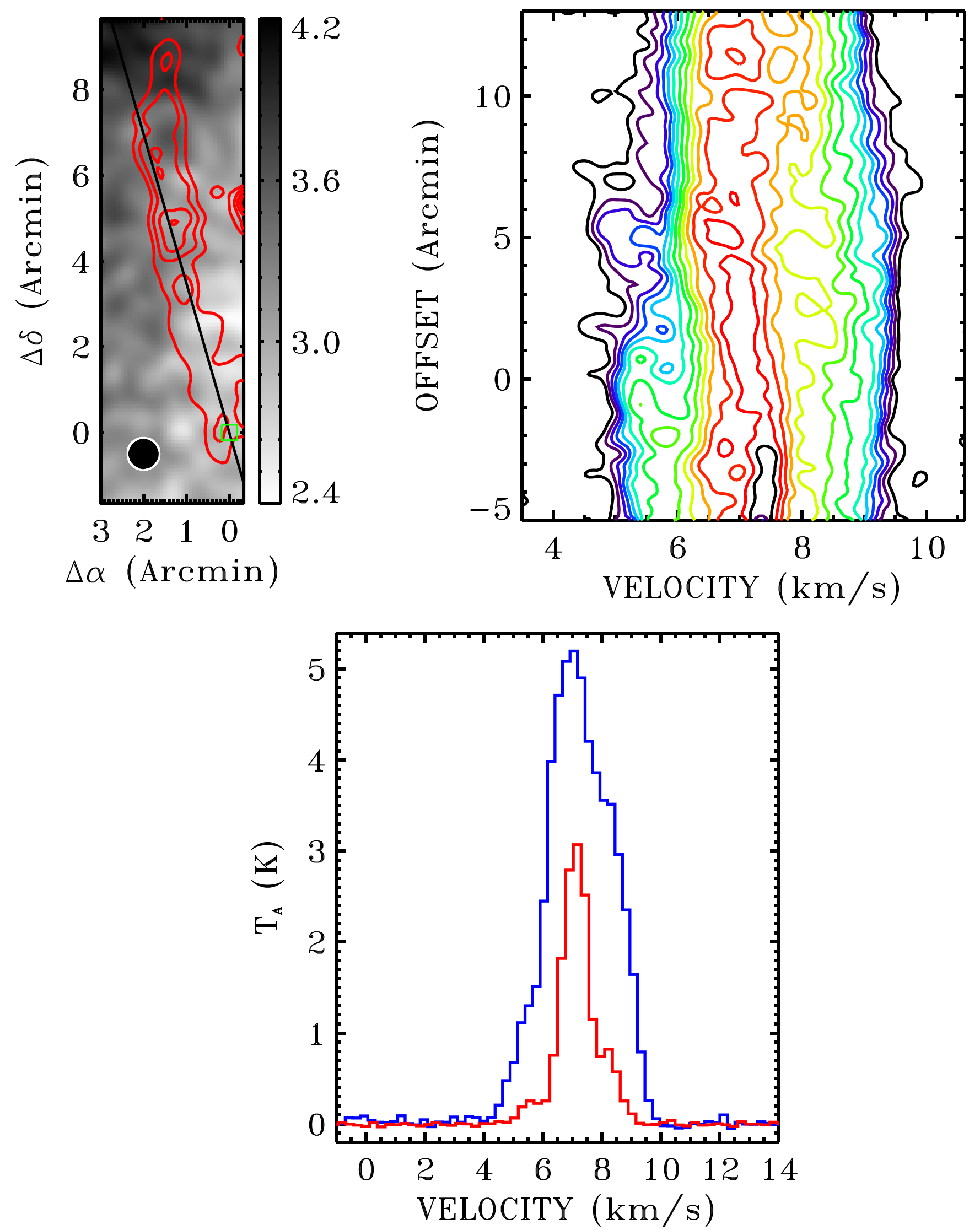

Figure 54. TMO_53 (SST 041841.3+282725). Same as Figure 3 except for the following. In the upper left panel, the integrated intervals for the red lobe and grayscale images are 9.3-9.9 $\mathrm{km} \mathrm{s}^{-1}$ and $6-8 \mathrm{~km} \mathrm{~s}^{-1}$, respectively. The red contour levels are $60 \%, 70 \%, \ldots, 90 \%$ of the peak value. In the upper right panel, the position angle is $164^{\circ}$ and the contour levels are $0.4-1.6 \mathrm{~K}$ by $0.3 \mathrm{~K}, 2-6 \mathrm{~K}$ by $0.5 \mathrm{~K}$. 

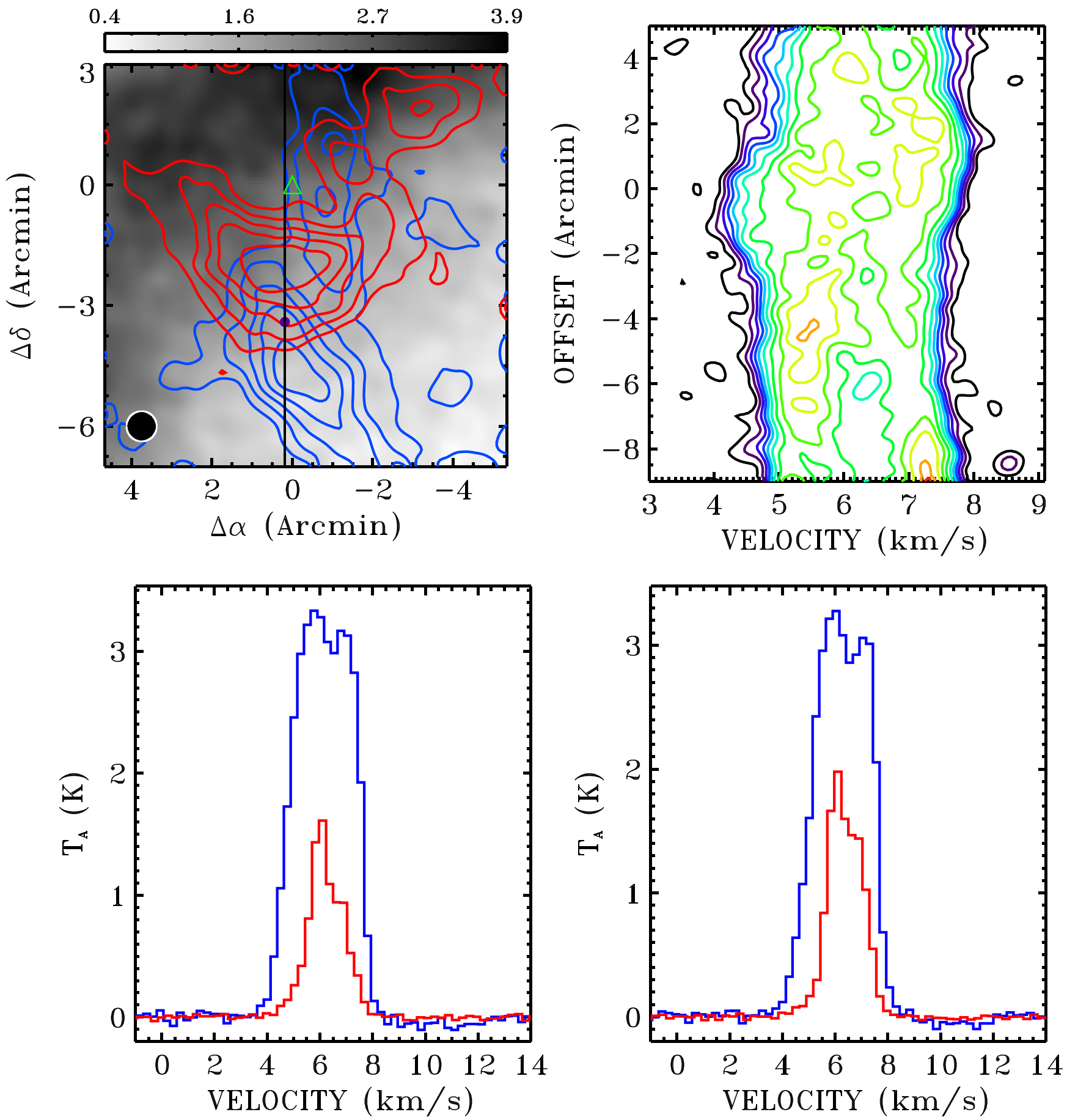

Figure 55. TMO 54 (SST 042154.5+265231). Same as Figure 2 except for the following. In the upper left panel, the integrated intervals for the blue lobe, red lobe, and grayscale images are $3.8-4.5 \mathrm{~km} \mathrm{~s}^{-1}, 7.5-8.3 \mathrm{~km} \mathrm{~s}^{-1}$, and $5.5-7 \mathrm{~km} \mathrm{~s}^{-1}$, respectively. The blue and red contour levels are $30 \%, 45 \%, \ldots, 90 \%$ and $50 \%, 60 \%, \ldots, 90 \%$ of their peak value, respectively. In the upper right panel, the position angle is $0^{\circ}$ and the contour levels are $0.5-2 \mathrm{~K}$ by $0.3 \mathrm{~K}, 2.5-4 \mathrm{~K}$ by $0.5 \mathrm{~K}$.

Equation (7), we determine the turbulent energy of Taurus to be $3.2 \times 10^{47} \mathrm{erg}$. The energy of all of the detected outflows $\left(3.9 \times 10^{45} \mathrm{erg}\right)$ is about two orders of magnitude less than the turbulent energy of the cloud. The lower limit of the total energy of the bubbles $\left(9.2 \times 10^{46} \mathrm{erg}\right)$ is $29 \%$ of the turbulent energy. We conclude that the total energy of outflows and bubbles cannot balance the turbulence in Taurus.

\subsubsection{The Turbulent Dissipation Rate is Comparable to the Bubble Energy Injection Rate}

We also estimated the total outflow energy rate (outflow luminosity, $L_{\text {flow }}$ ) and the total bubble energy rate (bubble luminosity, $L_{\text {bubble }}$ ) with the energy rate needed to maintain the turbulence (turbulent energy dissipation, $\left.L_{\text {turb }}\right)$. The luminosity of outflows is $1.3 \times 10^{33} \mathrm{erg} \mathrm{s}^{-1}$ after inclination and blending 

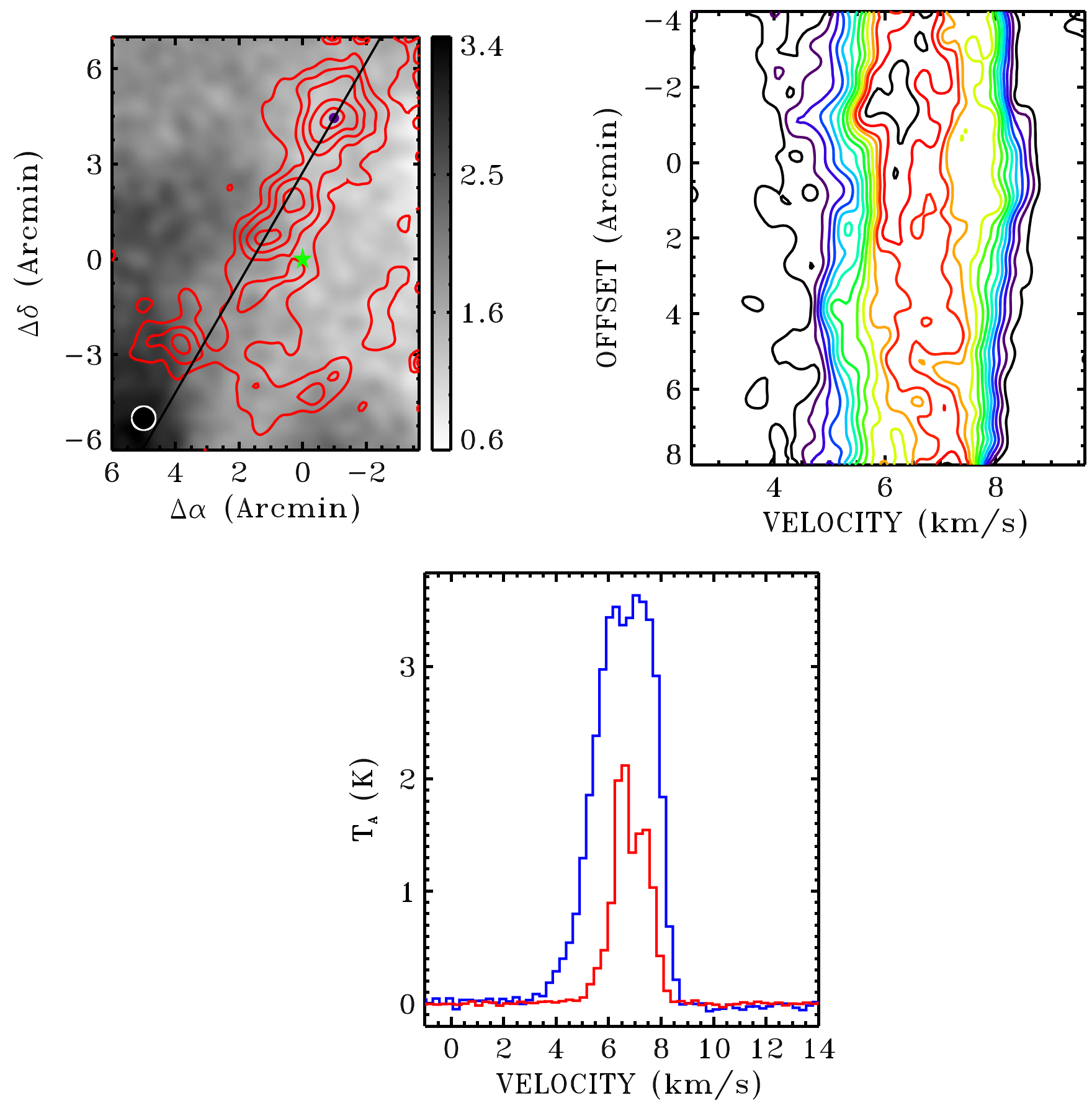

Figure 56. TMO_55 (SST 042904.9+264907). Same as Figure 3 except for the following. In the upper left panel, the integrated intervals for the red lobe and grayscale images are $8.3-9 \mathrm{~km} \mathrm{~s}^{-1}$ and $5.5-7 \mathrm{~km} \mathrm{~s}^{-1}$, respectively. In the upper right panel, the position angle is $50^{\circ}$ and the contour levels are $0.4-4.2 \mathrm{~K}$ by $0.3 \mathrm{~K}$.

correction. Summing up the luminosity in Table 5, we determine the energy injection rate of bubbles to be $6.4 \times 10^{33} \mathrm{erg} \mathrm{s}^{-1}$.

The turbulent dissipation rate can be calculated as

$$
L_{\text {turb }}=\frac{E_{\text {turb }}}{t_{\text {diss }}},
$$

where $t_{\text {diss }}$ is the turbulent dissipation time. We estimate the turbulent dissipation time through two methods based on numerical simulations.
First, the turbulent dissipation time of the cloud is given by (McKee \& Ostriker 2007)

$$
t_{\text {diss }} \sim 0.5 \frac{d}{\sigma_{1 \mathrm{~d}}},
$$

where $d=27.6 \mathrm{pc}$ is the cloud diameter and $\sigma_{1 \mathrm{~d}}$ is the one-dimensional turbulent velocity dispersion along the line of sight,

$$
\sigma_{1 \mathrm{~d}}=\frac{\Delta v_{\mathrm{FWHM}}}{2 \sqrt{2 \ln 2}}
$$



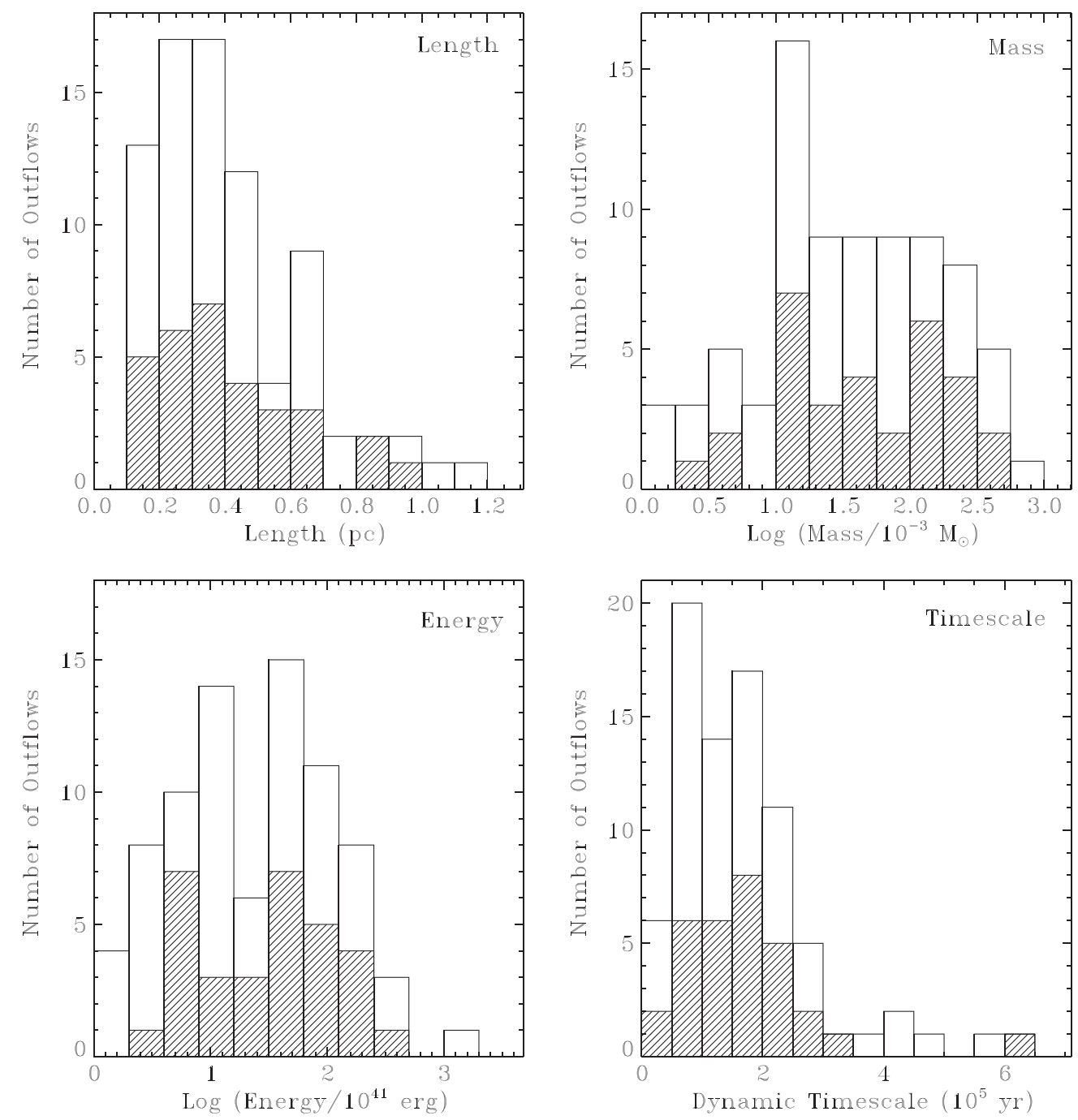

Figure 57. Histograms of the distributions of outflow parameters. The shaded histograms represent the blue lobes and the open histograms represent the red lobes.

Here $\Delta v_{\mathrm{FWHM}}=2 \mathrm{~km} \mathrm{~s}^{-1}$ is the same as that in Section 5.3.2. Then, we obtain $\sigma_{1 \mathrm{~d}}=0.85 \mathrm{~km} \mathrm{~s}^{-1}$. Combining Equations (10) and (11), we obtain the turbulent dissipation time, $t_{\text {diss }}=1.6 \times 10^{7}$ years, which is about six times larger than the result $\left(2.7 \times 10^{6}\right.$ years $)$ in Narayanan et al. (2012). Then, using Equation (9), we determine the turbulent dissipation rate to be $6.6 \times 10^{32} \mathrm{erg} \mathrm{s}^{-1}$, which is $51 \%$ of the luminosity of outflows and only $10 \%$ of the energy injection rate of bubbles.

Second, we follow Mac Low (1999) in calculating the dissipation time of the cloud by

$$
t_{\mathrm{diss}} \sim\left(\frac{3.9 \kappa}{M_{\mathrm{rms}}}\right) t_{\mathrm{ff}},
$$

where $t_{\mathrm{ff}}$ is the free-fall timescale, $M_{\mathrm{rms}}$ is the Mach number of the turbulence, and $\kappa$ is the ratio of the driving length to the Jean's length of the cloud,

$$
\kappa=\frac{\lambda_{\mathrm{d}}}{\lambda_{\mathrm{J}}}
$$

Using our extensive data sets, we obtain $\lambda_{\mathrm{d}}=0.53 \mathrm{pc}$, which is the average size of outflows and bubbles we found in Taurus. The Jean's length is defined as

$$
\lambda_{\mathrm{J}}=c_{\mathrm{s}}\left(\pi / G \rho_{\mathrm{reg}}\right)^{1 / 2},
$$

where $c_{\mathrm{s}}$ is the sound speed (Mac Low 1999). For an ideal gas

$$
c_{\mathrm{s}}=(3 k T / \mu)^{1 / 2},
$$

where $k$ is the Boltzmann's constant, $T=10 \mathrm{~K}$ is the temperature of the Taurus molecular cloud, and $\boldsymbol{\mu}=2.72$ is the mean molecular weight (Brunt 2010). Then, we get the sound speed, $c_{\mathrm{s}}=0.3 \mathrm{~km} \mathrm{~s}^{-1} \cdot \rho_{\text {reg }}$ is the representative volume density of the region where dissipation takes place. We estimate the volume density to be $\sim 1500 \mathrm{~cm}^{-3}$. Using Equation (14), we obtain the Jean's length of the region, $\lambda_{\mathrm{J}}=0.83 \mathrm{pc}$, which is about four times larger than that $\left(\lambda_{\mathrm{J}} \sim 0.2 \mathrm{pc}\right)$ in Perseus (Arce et al. 2010). Then, using Equation (13), we obtain $\kappa=0.64$, which is different from the assumption $(\kappa \sim 1)$ by Arce et al. (2010) and Narayanan et al. (2012). 

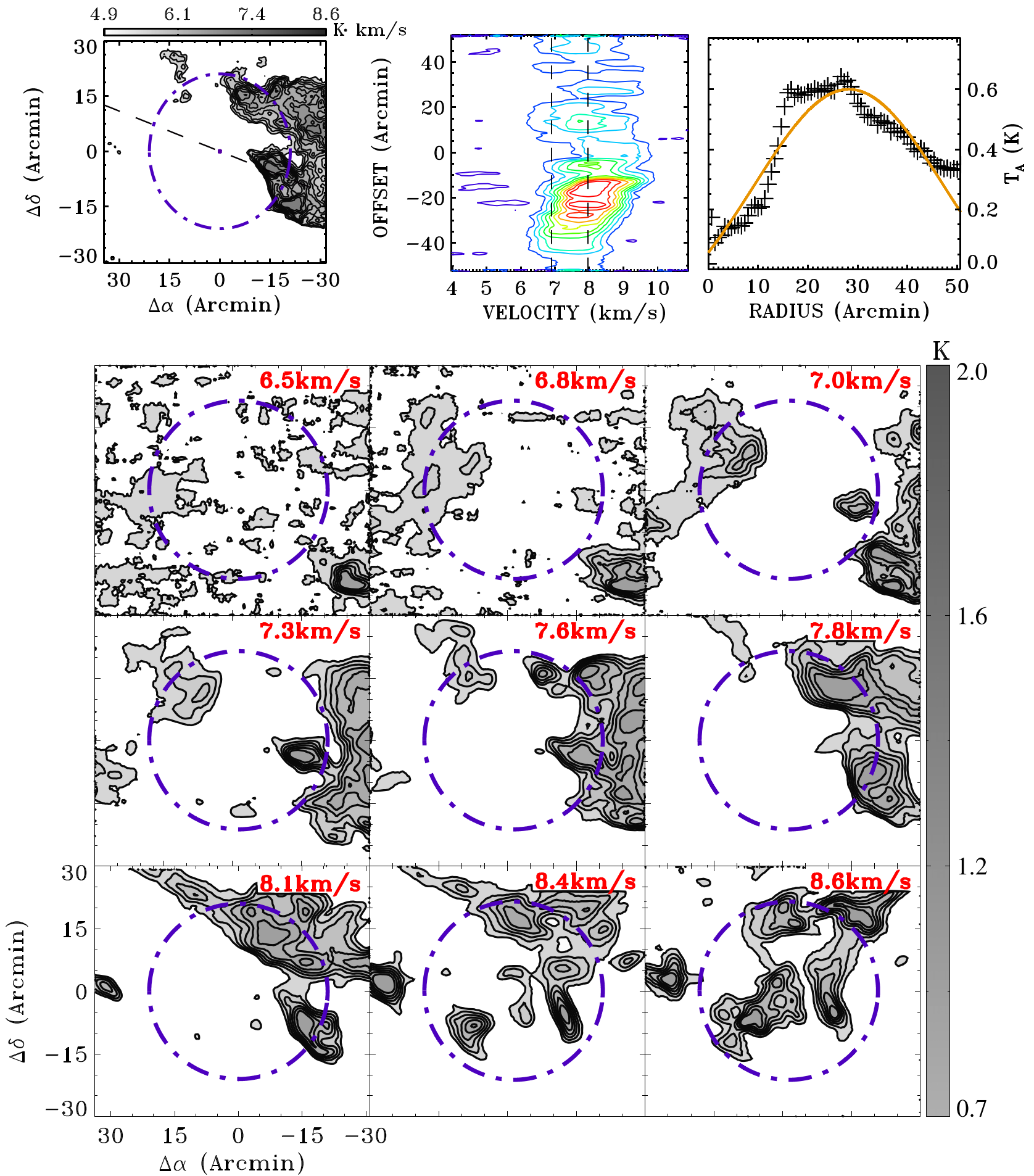

Figure 58. TMB 01. Upper left panel: ${ }^{13} \mathrm{CO}$ integrated intensity map integrated over $7.3-8.4 \mathrm{~km} \mathrm{~s}^{-1}$. The starting contour and the contour step are 3.3 and $0.6 \mathrm{~K} \mathrm{~km} \mathrm{~s}^{-1}$, respectively. The black dashed line represents a cut for the $\mathrm{P}-\mathrm{V}$ diagram shown in the upper middle panel, and the purple open circle indicates the origin on the $Y$-axis of the upper middle panel. The purple dashed circle shows the approximate extent of the $\mathrm{CO}$ emission of the bubble. The black open circle on the lower left corner marks the beam. Upper middle panel: $\mathrm{P}-\mathrm{V}$ diagram of ${ }^{13} \mathrm{CO}$ through the slice shown by the black dashed line in the upper left panel at a position angle of $110^{\circ}$. The contour levels are $0.1-1.2 \mathrm{~K}$ by $0.1 \mathrm{~K}$. The two black dashed vertical lines show the expansion velocity interval determined by visual inspection. Upper right panel: Gaussian fit to the azimuthally averaged profile of ${ }^{13} \mathrm{CO}$ intensity. Lower panel: channel maps of ${ }^{13} \mathrm{CO}$ emission; the number on the upper right corner of each panel indicates the central velocity of the channel map. The purple dashed circles are the same as that in the upper left panel. 

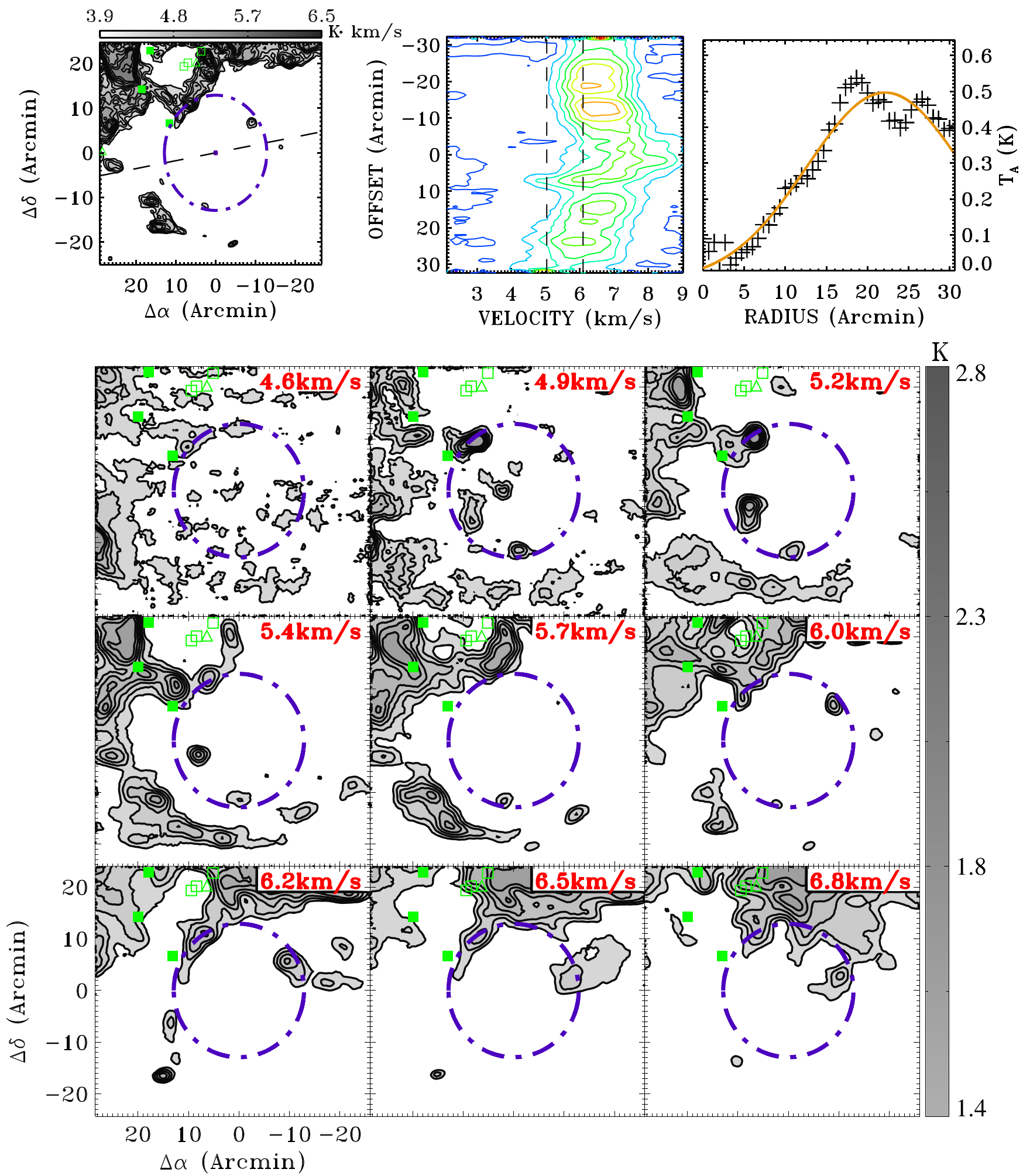

Figure 59. TMB_02. Same as Figure 58 except for the following. In the upper left panel, the integrated velocity interval is from 5.4 to $6.5 \mathrm{~km} \mathrm{~s}^{-1}$, the starting contour and the contour step are 2.7 and $0.5 \mathrm{~K} \mathrm{~km} \mathrm{~s}^{-1}$, respectively. In the upper middle panel, the position angle is $80^{\circ}$ and the contour levels are $0.1-1.0 \mathrm{~K}$ by $0.1 \mathrm{~K}$. 
Table 5

Physical Parameters of Outflows

\begin{tabular}{|c|c|c|c|c|c|c|c|c|c|}
\hline $\begin{array}{l}\text { Outflow } \\
\text { Name }\end{array}$ & Lobe & $\begin{array}{c}v_{\mathrm{avg}}^{\mathrm{a}} \\
\left(\mathrm{km} \mathrm{s}^{-1}\right)\end{array}$ & $\begin{array}{c}\text { Area }^{\mathrm{b}} \\
(\operatorname{arcmin})\end{array}$ & $\begin{array}{l}\text { Length } \\
\text { (pc) }\end{array}$ & $\begin{array}{l}\text { Mass } \\
\left(M_{\odot}\right)\end{array}$ & $\begin{array}{c}\text { Momentum } \\
\left(M_{\odot} \mathrm{km} \mathrm{s}^{-1}\right)\end{array}$ & $\begin{array}{c}\text { Energy } \\
\left(10^{43} \mathrm{erg}\right)\end{array}$ & $\begin{array}{c}t_{\text {dyn }} \\
\left(10^{5} \text { years }\right)\end{array}$ & $\begin{array}{c}L_{\text {flow }} \\
\left(10^{30} \mathrm{erg} \mathrm{s}^{-1}\right)\end{array}$ \\
\hline \multirow[t]{2}{*}{ TMO_01 } & Blueshifted & $\ldots$ & $\ldots$ & $\cdots$ & $\ldots$ & $\ldots$ & $\ldots$ & $\cdots$ & $\ldots$ \\
\hline & Redshifted & 2.5 & $11 \times 25$ & 1.11 & 0.083 & 0.205 & 0.51 & 4.4 & 0.37 \\
\hline \multirow[t]{2}{*}{ TMO_02 } & Blueshifted & 3.4 & $8 \times 12$ & 0.58 & 0.168 & 0.570 & 1.92 & 1.7 & 3.63 \\
\hline & Redshifted & 3.6 & $6 \times 5$ & 0.31 & 0.061 & 0.220 & 0.79 & 0.8 & 2.97 \\
\hline \multirow[t]{2}{*}{ TMO_03 } & Blueshifted & 3.1 & $6 \times 9$ & 0.41 & 0.050 & 0.156 & 0.49 & 1.3 & 1.19 \\
\hline & Redshifted & 2.4 & $11 \times 2$ & 0.46 & 0.013 & 0.030 & 0.07 & 1.9 & 0.12 \\
\hline \multirow[t]{2}{*}{ TMO_04 } & Blueshifted & 2.6 & $6 \times 8$ & 0.41 & 0.026 & 0.069 & 0.18 & 1.5 & 0.36 \\
\hline & Redshifted & 2.5 & $3 \times 8$ & 0.33 & 0.005 & 0.013 & 0.03 & 1.3 & 0.08 \\
\hline \multirow[t]{2}{*}{ TMO_05 } & Blueshifted & 4.6 & $9 \times 18$ & 0.83 & 0.078 & 0.355 & 1.61 & 1.8 & 2.89 \\
\hline & Redshifted & $\cdots$ & $\cdots$ & $\cdots$ & $\cdots$ & $\cdots$ & $\cdots$ & $\cdots$ & $\cdots$ \\
\hline \multirow[t]{2}{*}{ TMO_06 } & Blueshifted & 2.8 & $5 \times 5$ & 0.29 & 0.038 & 0.109 & 0.31 & 1.0 & 0.96 \\
\hline & Redshifted & 3.9 & $3 \times 5$ & 0.21 & 0.010 & 0.038 & 0.15 & 0.5 & 0.88 \\
\hline \multirow{2}{*}{ TMO_07 } & Blueshifted & 2.1 & $7 \times 7$ & 0.38 & 0.012 & 0.025 & 0.05 & 1.8 & 0.09 \\
\hline & Redshifted & 2.7 & $2 \times 2$ & 0.13 & 0.002 & 0.005 & 0.01 & 0.5 & 0.08 \\
\hline \multirow[t]{2}{*}{ TMO_08 } & Blueshifted & 3.1 & $5 \times 4$ & 0.26 & 0.013 & 0.039 & 0.12 & 0.8 & 0.48 \\
\hline & Redshifted & 2.8 & $2 \times 2$ & 0.12 & 0.001 & 0.003 & 0.01 & 0.4 & 0.07 \\
\hline \multirow[t]{2}{*}{ TMO_09 } & Blueshifted & $\ldots$ & $\ldots$ & $\ldots$ & $\ldots$ & $\ldots$ & $\ldots$ & $\ldots$ & $\ldots$ \\
\hline & Redshifted & 1.8 & $17 \times 18$ & 1.01 & 0.311 & 0.565 & 1.02 & 5.5 & 0.59 \\
\hline \multirow[t]{2}{*}{ TMO_10 } & Blueshifted & 2.3 & $3 \times 7$ & 0.29 & 0.010 & 0.023 & 0.05 & 1.3 & 0.13 \\
\hline & Redshifted & 2.9 & $7 \times 3$ & 0.30 & 0.016 & 0.047 & 0.14 & 1.0 & 0.43 \\
\hline \multirow{2}{*}{$\mathrm{TMO}_{-} 11$} & Blueshifted & $\cdots$ & $\cdots$ & $\cdots$ & $\cdots$ & $\cdots$ & $\cdots$ & $\cdots$ & $\cdots$ \\
\hline & Redshifted & 2.6 & $7 \times 7$ & 0.40 & 0.020 & 0.052 & 0.14 & 1.5 & 0.29 \\
\hline \multirow[t]{2}{*}{ TMO_12 } & Blueshifted & $\cdots$ & $\cdots$ & $\cdots$ & $\cdots$ & $\cdots$ & $\cdots$ & $\cdots$ & $\cdots$ \\
\hline & Redshifted & 2.5 & $4 \times 3$ & 0.22 & 0.014 & 0.036 & 0.09 & 0.8 & 0.33 \\
\hline \multirow[t]{2}{*}{ TMO_13 } & Blueshifted & $\cdots$ & $\cdots$ & $\cdots$ & $\cdots$ & $\cdots$ & $\cdots$ & $\cdots$ & $\cdots$ \\
\hline & Redshifted & 2.2 & $7 \times 4$ & 0.34 & 0.053 & 0.115 & 0.25 & 1.5 & 0.51 \\
\hline TMO_14 & Blueshifted & 2.6 & $5 \times 5$ & 0.29 & 0.019 & 0.050 & 0.13 & 1.1 & 0.38 \\
\hline & Redshifted & 3.2 & $7 \times 4$ & 0.31 & 0.031 & 0.099 & 0.32 & 0.9 & 1.05 \\
\hline TMO_15 & Blueshifted & 1.9 & $3 \times 3$ & 0.18 & 0.013 & 0.024 & 0.05 & 0.9 & 0.16 \\
\hline & Redshifted & 1.5 & $3 \times 4$ & 0.20 & 0.010 & 0.016 & 0.02 & 1.3 & 0.06 \\
\hline TMO_16 & Blueshifted & 2.3 & $2 \times 2$ & 0.11 & 0.005 & 0.012 & 0.03 & 0.4 & 0.21 \\
\hline & Redshifted & 2.0 & $4 \times 4$ & 0.23 & 0.023 & 0.046 & 0.09 & 1.1 & 0.25 \\
\hline TMO_17 & Blueshifted & $\cdots$ & $\cdots$ & $\cdots$ & $\ldots$ & $\cdots$ & $\cdots$ & $\cdots$ & $\cdots$ \\
\hline & Redshifted & 1.7 & $5 \times 14$ & 0.60 & 0.042 & 0.072 & 0.12 & 3.5 & 0.11 \\
\hline TMO_18 & Blueshifted & 2.0 & $10 \times 3$ & 0.42 & 0.012 & 0.024 & 0.05 & 2.1 & 0.07 \\
\hline & Redshifted & 1.9 & $3 \times 4$ & 0.20 & 0.007 & 0.013 & 0.02 & 1.0 & 0.08 \\
\hline TMO_19 & Blueshifted & $\cdots$ & $\cdots$ & $\cdots$ & $\cdots$ & $\cdots$ & $\cdots$ & $\cdots$ & $\cdots$ \\
\hline & Redshifted & 2.2 & $4 \times 4$ & 0.23 & 0.008 & 0.017 & 0.04 & 1.0 & 0.11 \\
\hline TMO_20 & Blueshifted & $\ldots$ & $\cdots$ & $\cdots$ & $\cdots$ & $\cdots$ & $\cdots$ & $\cdots$ & $\cdots$ \\
\hline & Redshifted & 4.0 & $7 \times 3$ & 0.31 & 0.017 & 0.067 & 0.26 & 0.8 & 1.08 \\
\hline TMO_21 & Blueshifted & 3.1 & $4 \times 2$ & 0.17 & 0.004 & 0.012 & 0.04 & 0.5 & 0.22 \\
\hline & Redshifted & 2.9 & $5 \times 3$ & 0.22 & 0.011 & 0.032 & 0.09 & 0.7 & 0.40 \\
\hline TMO_22 & Blueshifted & 1.6 & $10 \times 22$ & 0.97 & 0.226 & 0.351 & 0.54 & 6.1 & 0.28 \\
\hline & Redshifted & $\ldots$ & $\ldots$ & $\ldots$ & $\ldots$ & $\cdots$ & $\ldots$ & $\ldots$ & $\ldots$ \\
\hline TMO_23 & Blueshifted & $\cdots$ & $\cdots$ & $\cdots$ & $\cdots$ & $\cdots$ & $\cdots$ & $\cdots$ & $\cdots$ \\
\hline & Redshifted & 3.7 & $3 \times 6$ & 0.27 & 0.019 & 0.072 & 0.26 & 0.7 & 1.18 \\
\hline TMO_24 & Blueshifted & $\cdots$ & $\cdots$ & $\cdots$ & $\cdots$ & $\cdots$ & $\cdots$ & $\cdots$ & $\cdots$ \\
\hline & Redshifted & 3.2 & $4 \times 6$ & 0.29 & 0.015 & 0.047 & 0.15 & 0.9 & 0.52 \\
\hline TMO_25 & Blueshifted & 2.4 & $5 \times 5$ & 0.31 & 0.267 & 0.640 & 1.52 & 1.2 & 3.87 \\
\hline & Redshifted & $\cdots$ & $\cdots$ & $\cdots$ & $\cdots$ & $\cdots$ & $\cdots$ & $\cdots$ & $\cdots$ \\
\hline TMO_26 & Blueshifted & $\cdots$ & $\cdots$ & $\ldots$ & $\ldots$ & $\cdots$ & $\cdots$ & $\cdots$ & $\cdots$ \\
\hline & Redshifted & 2.4 & $13 \times 8$ & 0.60 & 0.369 & 0.880 & 2.09 & 2.5 & 2.68 \\
\hline TMO_27 & Blueshifted & $\cdots$ & $\cdots$ & $\cdots$ & $\cdots$ & $\cdots$ & $\cdots$ & $\cdots$ & $\cdots$ \\
\hline & Redshifted & 2.4 & $6 \times 7$ & 0.37 & 0.074 & 0.174 & 0.41 & 1.5 & 0.86 \\
\hline TMO_28 & Blueshifted & 2.2 & $4 \times 6$ & 0.30 & 0.170 & 0.371 & 0.80 & 1.3 & 1.91 \\
\hline & Redshifted & 3.9 & $3 \times 2$ & 0.15 & 0.031 & 0.122 & 0.47 & 0.4 & 3.80 \\
\hline TMO_29 & Blueshifted & 1.9 & $9 \times 4$ & 0.40 & 0.264 & 0.492 & 0.91 & 2.1 & 1.37 \\
\hline & Redshifted & 2.1 & $6 \times 5$ & 0.32 & 0.076 & 0.161 & 0.34 & 1.5 & 0.74 \\
\hline TMO_30 & Blueshifted & $\ldots$ & $\ldots$ & $\ldots$ & $\ldots$ & $\ldots$ & $\ldots$ & $\ldots$ & $\ldots$ \\
\hline & Redshifted & 2.4 & $9 \times 9$ & 0.49 & 0.286 & 0.673 & 1.57 & 2.1 & 2.43 \\
\hline TMO_31 & Blueshifted & $\cdots$ & $\cdots$ & $\cdots$ & $\cdots$ & $\cdots$ & $\cdots$ & $\cdots$ & $\cdots$ \\
\hline & Redshifted & 2.1 & $5 \times 14$ & 0.62 & 0.277 & 0.587 & 1.24 & 2.9 & 1.37 \\
\hline
\end{tabular}


Table 5

(Continued)

\begin{tabular}{|c|c|c|c|c|c|c|c|c|c|}
\hline $\begin{array}{l}\text { Outflow } \\
\text { Name }\end{array}$ & Lobe & $\begin{array}{c}v_{\mathrm{avg}}{ }^{\mathrm{a}} \\
\left(\mathrm{km} \mathrm{s}^{-1}\right)\end{array}$ & $\begin{array}{c}\text { Area }^{\mathrm{b}} \\
(\operatorname{arcmin})\end{array}$ & $\begin{array}{l}\text { Length } \\
\text { (pc) }\end{array}$ & $\begin{array}{l}\text { Mass } \\
\left(M_{\odot}\right)\end{array}$ & $\begin{array}{c}\text { Momentum } \\
\left(M_{\odot} \mathrm{km} \mathrm{s}^{-1}\right)\end{array}$ & $\begin{array}{c}\text { Energy } \\
\left(10^{43} \mathrm{erg}\right)\end{array}$ & $\begin{array}{c}t_{\text {dyn }} \\
\left(10^{5} \text { years }\right)\end{array}$ & $\begin{array}{c}L_{\text {flow }} \\
\left(10^{30} \mathrm{erg} \mathrm{s}^{-1}\right)\end{array}$ \\
\hline \multirow[t]{2}{*}{ TMO_32 } & Blueshifted & $\ldots$ & $\ldots$ & $\ldots$ & $\ldots$ & $\ldots$ & $\ldots$ & $\ldots$ & $\ldots$ \\
\hline & Redshifted & 3.3 & $9 \times 6$ & 0.42 & 0.131 & 0.429 & 1.39 & 1.2 & 3.53 \\
\hline \multirow[t]{2}{*}{ TMO_33 } & Blueshifted & 2.1 & $8 \times 5$ & 0.38 & 0.120 & 0.249 & 0.51 & 1.8 & 0.90 \\
\hline & Redshifted & 3.7 & $11 \times 13$ & 0.68 & 0.896 & 3.299 & 12.08 & 1.8 & 1.174 \\
\hline \multirow[t]{2}{*}{$\mathrm{TMO}_{-} 34$} & Blueshifted & 3.2 & $5 \times 3$ & 0.23 & 0.061 & 0.198 & 0.63 & 0.7 & 2.86 \\
\hline & Redshifted & $\ldots$ & $\ldots$ & $\ldots$ & $\ldots$ & $\ldots$ & $\ldots$ & $\ldots$ & $\ldots$ \\
\hline \multirow[t]{2}{*}{ TMO_35 } & Blueshifted & $\ldots$ & $\ldots$ & $\ldots$ & $\ldots$ & $\ldots$ & $\ldots$ & $\ldots$ & $\ldots$ \\
\hline & Redshifted & 3.2 & $6 \times 4$ & 0.28 & 0.049 & 0.156 & 0.49 & 0.9 & 1.79 \\
\hline \multirow[t]{2}{*}{ TMO_36 } & Blueshifted & 2.6 & $9 \times 14$ & 0.65 & 0.484 & 1.241 & 3.17 & 2.5 & 4.03 \\
\hline & Redshifted & 1.9 & $8 \times 6$ & 0.42 & 0.092 & 0.177 & 0.34 & 2.1 & 0.51 \\
\hline \multirow[t]{2}{*}{ TMO_37 } & Blueshifted & $\ldots$ & $\ldots$ & $\ldots$ & $\ldots$ & $\ldots$ & $\ldots$ & $\ldots$ & $\ldots$ \\
\hline & Redshifted & 2.1 & $15 \times 19$ & 0.99 & 0.348 & 0.737 & 1.55 & 4.6 & 1.08 \\
\hline \multirow[t]{2}{*}{ TMO_38 } & Blueshifted & 2.3 & $13 \times 8$ & 0.61 & 0.377 & 0.860 & 1.95 & 2.6 & 2.35 \\
\hline & Redshifted & $\ldots$ & $\ldots$ & $\ldots$ & $\ldots$ & $\ldots$ & $\ldots$ & $\ldots$ & $\ldots$ \\
\hline \multirow[t]{2}{*}{ TMO_39 } & Blueshifted & 2.3 & $7 \times 5$ & 0.35 & 0.136 & 0.313 & 0.72 & 1.5 & 1.51 \\
\hline & Redshifted & 2.2 & $6 \times 5$ & 0.32 & 0.076 & 0.167 & 0.37 & 1.4 & 0.81 \\
\hline \multirow{2}{*}{$\mathrm{TMO}_{-} 40$} & Blueshifted & $\ldots$ & $\ldots$ & $\ldots$ & $\ldots$ & $\ldots$ & $\ldots$ & $\ldots$ & $\cdots$ \\
\hline & Redshifted & 4.7 & $4 \times 4$ & 0.25 & 0.129 & 0.607 & 2.84 & 0.5 & 7.683 \\
\hline \multirow[t]{2}{*}{$\mathrm{TMO}_{-} 41$} & Blueshifted & 3.8 & $3 \times 4$ & 0.23 & 0.028 & 0.104 & 0.39 & 0.6 & 2.09 \\
\hline & Redshifted & 3.9 & $5 \times 15$ & 0.63 & 0.317 & 1.226 & 4.72 & 1.6 & 9.401 \\
\hline \multirow[t]{2}{*}{$\mathrm{TMO}_{-} 42$} & Blueshifted & 3.2 & $5 \times 15$ & 0.65 & 0.105 & 0.338 & 1.08 & 2.0 & 1.72 \\
\hline & Redshifted & 2.3 & $3 \times 3$ & 0.17 & 0.004 & 0.010 & 0.02 & 0.7 & 0.10 \\
\hline \multirow[t]{2}{*}{ TMO_43 } & Blueshifted & 2.7 & $9 \times 9$ & 0.51 & 0.034 & 0.092 & 0.24 & 1.9 & 0.41 \\
\hline & Redshifted & $\cdots$ & $\ldots$ & $\ldots$ & $\ldots$ & $\cdots$ & $\ldots$ & $\cdots$ & $\ldots$ \\
\hline \multirow[t]{2}{*}{$\mathrm{TMO}_{-} 44$} & Blueshifted & 2.4 & $15 \times 14$ & 0.84 & 0.148 & 0.359 & 0.87 & 3.4 & 0.81 \\
\hline & Redshifted & 1.4 & $9 \times 13$ & 0.63 & 0.070 & 0.097 & 0.13 & 4.4 & 0.10 \\
\hline \multirow[t]{2}{*}{ TMO_45 } & Blueshifted & 2.9 & $2 \times 4$ & 0.19 & 0.013 & 0.037 & 0.11 & 0.6 & 0.53 \\
\hline & Redshifted & 2.1 & $3 \times 2$ & 0.14 & 0.006 & 0.013 & 0.03 & 0.6 & 0.14 \\
\hline \multirow[t]{2}{*}{ TMO_46 } & Blueshifted & 2.8 & $6 \times 13$ & 0.58 & 0.050 & 0.141 & 0.39 & 2.0 & 0.61 \\
\hline & Redshifted & 2.9 & $5 \times 18$ & 0.76 & 0.123 & 0.362 & 1.06 & 2.5 & 1.34 \\
\hline \multirow[t]{2}{*}{ TMO_47 } & Blueshifted & 1.5 & $8 \times 3$ & 0.36 & 0.193 & 0.282 & 0.41 & 2.4 & 0.53 \\
\hline & Redshifted & 1.9 & $5 \times 10$ & 0.45 & 0.304 & 0.583 & 1.11 & 2.3 & 1.53 \\
\hline \multirow[t]{2}{*}{ TMO_48 } & Blueshifted & 5.2 & $2 \times 3$ & 0.15 & 0.002 & 0.012 & 0.06 & 0.3 & 0.71 \\
\hline & Redshifted & 3.4 & $2 \times 2$ & 0.11 & 0.001 & 0.004 & 0.02 & 0.3 & 0.16 \\
\hline \multirow[t]{2}{*}{ TMO_49 } & Blueshifted & $\ldots$ & $\ldots$ & $\ldots$ & $\ldots$ & $\ldots$ & $\ldots$ & $\ldots$ & $\ldots$ \\
\hline & Redshifted & 4.4 & $7 \times 17$ & 0.76 & 0.042 & 0.182 & 0.79 & 1.7 & 1.47 \\
\hline \multirow[t]{2}{*}{ TMO_50 } & Blueshifted & $\ldots$ & $\ldots$ & $\ldots$ & $\ldots$ & $\ldots$ & $\ldots$ & $\ldots$ & $\ldots$ \\
\hline & Redshifted & 2.2 & $4 \times 13$ & 0.55 & 0.020 & 0.044 & 0.10 & 2.4 & 0.12 \\
\hline \multirow[t]{2}{*}{ TMO_51 } & Blueshifted & $\ldots$ & $\ldots$ & $\ldots$ & $\ldots$ & $\ldots$ & $\ldots$ & $\ldots$ & $\ldots$ \\
\hline & Redshifted & 2.9 & $2 \times 2$ & 0.10 & 0.001 & 0.003 & 0.01 & 0.4 & 0.08 \\
\hline TMO_52 & Blueshifted & $\ldots$ & $\ldots$ & $\ldots$ & $\ldots$ & $\ldots$ & $\ldots$ & $\ldots$ & $\ldots$ \\
\hline & Redshifted & 2.3 & $2 \times 3$ & 0.16 & 0.002 & 0.004 & 0.01 & 0.7 & 0.04 \\
\hline TMO_53 & Blueshifted & $\ldots$ & $\ldots$ & $\ldots$ & $\ldots$ & $\ldots$ & $\ldots$ & $\ldots$ & $\ldots$ \\
\hline & Redshifted & 2.4 & $2 \times 10$ & 0.41 & 0.005 & 0.011 & 0.03 & 1.7 & 0.05 \\
\hline TMO_54 & Blueshifted & 1.9 & $5 \times 5$ & 0.30 & 0.013 & 0.025 & 0.05 & 1.5 & 0.10 \\
\hline & Redshifted & 1.8 & $8 \times 5$ & 0.38 & 0.034 & 0.061 & 0.11 & 2.1 & 0.17 \\
\hline TMO_55 & Blueshifted & $\ldots$ & $\ldots$ & $\ldots$ & $\ldots$ & $\ldots$ & $\ldots$ & $\ldots$ & $\ldots$ \\
\hline & Redshifted & 2.0 & $4 \times 11$ & 0.49 & 0.011 & 0.023 & 0.05 & 2.4 & 0.06 \\
\hline
\end{tabular}

Notes.

${ }^{\mathrm{a}}$ The average velocity of the outflow relative to the cloud systemic velocity.

b The extent along R.A. $\times$ the extent along the decl. 
Table 6

Criteria for Bubble Classification and Bubble Distribution

\begin{tabular}{|c|c|c|c|c|c|c|}
\hline $\begin{array}{l}\text { Bubble } \\
\text { Class }\end{array}$ & $\begin{array}{c}\text { Contour } \\
\text { Map }\end{array}$ & $\begin{array}{c}\mathrm{P}-\mathrm{V} \\
\text { Diagram }\end{array}$ & $\begin{array}{c}\text { Channel } \\
\text { Maps }\end{array}$ & $\begin{array}{l}\text { Fitting } \\
\text { Curve }\end{array}$ & $\begin{array}{c}\text { Bubble } \\
\text { Numbers }\end{array}$ & Percentage \\
\hline A & $x$ & $x$ & $x$ & $x$ & 13 & $35.2 \%$ \\
\hline B1 & $\ldots$ & $x$ & $x$ & $\ldots$ & 6 & $16.2 \%$ \\
\hline B2 & $x$ & $\ldots$ & $x$ & $\ldots$ & 4 & $10.8 \%$ \\
\hline B3 & $x$ & $x$ & $\ldots$ & $\ldots$ & 4 & $10.8 \%$ \\
\hline B4 & $\ldots$ & $\ldots$ & $x$ & $\ldots$ & 4 & $10.8 \%$ \\
\hline $\mathrm{C}$ & $x$ & $\ldots$ & $\ldots$ & $\ldots$ & 6 & $16.2 \%$ \\
\hline
\end{tabular}

Table 7

Bubbles in Taurus

\begin{tabular}{|c|c|c|c|c|c|c|c|c|c|c|c|c|}
\hline Bubble & (J2000) & (J2000) & Bubble & YSO & Radius & $\begin{array}{c}V_{\exp } \\
\left(\mathrm{km} \mathrm{s}^{-1}\right)\end{array}$ & $\left(M_{\odot}\right)$ & $\begin{array}{l}\text { Momentum } \\
\left(M_{\odot} \mathrm{km} \mathrm{s}^{-1}\right)\end{array}$ & $\begin{array}{c}\text { Energy } \\
\left(10^{45} \mathrm{erg}\right)\end{array}$ & $\begin{array}{c}t_{\text {kinetic }} \\
10^{6} \text { years }\end{array}$ & $\begin{array}{c}L_{\text {bubble }} \\
\left(10^{32} \mathrm{erg} \mathrm{s}^{-1}\right)\end{array}$ & $\begin{array}{c}\dot{m}_{\mathrm{w}}{ }^{\mathrm{a}} \\
\left(10^{-8} M_{\odot}\right. \\
\left.\mathrm{yr}^{-1}\right)\end{array}$ \\
\hline TMB_01 & 041208 & 245333 & A & $\mathrm{N}$ & 0.98 & 1.3 & 25 & 31 & 0.39 & 0.8 & 0.17 & 15.5 \\
\hline TMB_02 & 041428 & 274553 & B1 & $\mathrm{Y}$ & 0.60 & 1.3 & 6 & 7 & 0.09 & 0.5 & 0.06 & 3.5 \\
\hline TMB_03 & 041620 & 282853 & A & $\mathrm{Y}$ & 0.76 & 1.8 & 10 & 18 & 0.33 & 0.4 & 0.25 & 9.0 \\
\hline TMB_04 & 041905 & 273333 & A & $\mathrm{Y}$ & 0.62 & 1.3 & 7 & 9 & 0.12 & 0.5 & 0.08 & 4.5 \\
\hline TMB_05 & 042112 & 265533 & B3 & $\mathrm{Y}$ & 0.56 & 1.8 & 4 & 8 & 0.14 & 0.3 & 0.14 & 4.0 \\
\hline TMB_06 & 042517 & 253213 & B1 & $\mathrm{N}$ & 0.77 & 1.3 & 10 & 12 & 0.16 & 0.6 & 0.08 & 6.0 \\
\hline TMB_07 & 042529 & 261013 & B1 & $\mathrm{N}$ & 1.12 & 2.3 & 102 & 234 & 5.31 & 0.5 & 3.51 & 117.0 \\
\hline TMB_08 & 042707 & 242013 & A & $\mathrm{N}$ & 0.70 & 2.3 & 18 & 42 & 0.95 & 0.3 & 1.00 & 21.0 \\
\hline TMB_09 & 042731 & 261653 & B4 & $\mathrm{N}$ & 0.49 & 1.0 & 4 & 4 & 0.04 & 0.5 & 0.03 & 2.0 \\
\hline TMB_10 & 042852 & 241433 & A & $\mathrm{Y}$ & 1.58 & 1.5 & 213 & 325 & 4.92 & 1.0 & 1.53 & 162.5 \\
\hline TMB_11 & 042932 & 263233 & A & $\mathrm{Y}$ & 0.84 & 2.0 & 41 & 83 & 1.68 & 0.4 & 1.31 & 41.5 \\
\hline TMB_12 & 042944 & 263253 & B4 & $\mathrm{Y}$ & 0.70 & 2.5 & 23 & 58 & 1.47 & 0.3 & 1.72 & 29.0 \\
\hline TMB_13 & 043031 & 242613 & A & $\mathrm{Y}$ & 0.73 & 2.3 & 18 & 41 & 0.93 & 0.3 & 0.94 & 20.5 \\
\hline TMB_14 & 043114 & 292553 & B2 & $\mathrm{Y}$ & 0.84 & 2.0 & 10 & 20 & 0.40 & 0.4 & 0.31 & 10.0 \\
\hline TMB_15 & 043130 & 241433 & A & $\mathrm{Y}$ & 0.84 & 1.3 & 34 & 43 & 0.54 & 0.6 & 0.27 & 21.5 \\
\hline TMB_16 & 043132 & 240953 & B3 & $\mathrm{N}$ & 0.70 & 1.8 & 22 & 39 & 0.69 & 0.4 & 0.57 & 19.5 \\
\hline TMB_17 & 043135 & 233513 & B4 & $\mathrm{N}$ & 0.42 & 1.0 & 2 & 2 & 0.02 & 0.4 & 0.02 & 1.0 \\
\hline TMB_18 & 043150 & 242213 & $\mathrm{C}$ & $\mathrm{N}$ & 0.56 & 2.0 & 14 & 29 & 0.59 & 0.3 & 0.69 & 14.5 \\
\hline TMB_19 & 043159 & 254313 & B1 & $\mathrm{Y}$ & 0.70 & 2.0 & 16 & 32 & 0.64 & 0.3 & 0.60 & 16.0 \\
\hline TMB_20 & 043203 & 253653 & $\mathrm{~B} 2$ & $\mathrm{~N}$ & 0.42 & 1.3 & 4 & 5 & 0.07 & 0.3 & 0.07 & 2.5 \\
\hline TMB_21 & 043237 & 292913 & A & $\mathrm{N}$ & 0.62 & 2.3 & 6 & 14 & 0.31 & 0.3 & 0.37 & 7.0 \\
\hline TMB_22 & 043239 & 244613 & A & $\mathrm{Y}$ & 1.06 & 1.3 & 28 & 36 & 0.45 & 0.8 & 0.17 & 18.0 \\
\hline TMB_23 & 043310 & 260853 & B4 & $\mathrm{N}$ & 0.28 & 1.3 & 2 & 2 & 0.03 & 0.2 & 0.04 & 1.0 \\
\hline TMB_24 & 043313 & 252453 & $\mathrm{C}$ & $\mathrm{N}$ & 0.49 & 1.3 & 5 & 7 & 0.09 & 0.4 & 0.07 & 3.5 \\
\hline TMB_25 & 043334 & 242053 & A & $\mathrm{Y}$ & 0.28 & 3.0 & 5 & 15 & 0.46 & 0.1 & 1.61 & 7.5 \\
\hline TMB_26 & 043447 & 293713 & B3 & $\mathrm{N}$ & 0.46 & 2.3 & 3 & 6 & 0.14 & 0.2 & 0.23 & 3.0 \\
\hline TMB_27 & 043602 & 282313 & $\mathrm{C}$ & $\mathrm{Y}$ & 1.40 & 2.5 & 64 & 161 & 4.08 & 0.5 & 2.39 & 80.5 \\
\hline TMB_28 & 043623 & 253633 & $\mathrm{~B} 2$ & $\mathrm{Y}$ & 1.40 & 3.3 & 386 & 1275 & 41.84 & 0.4 & 31.93 & 637.5 \\
\hline TMB_29 & 043704 & 254633 & $\mathrm{C}$ & $\mathrm{Y}$ & 0.84 & 1.5 & 20 & 30 & 0.46 & 0.5 & 0.27 & 15.0 \\
\hline TMB_30 & 043811 & 260553 & B3 & $\mathrm{Y}$ & 1.90 & 1.8 & 340 & 604 & 10.68 & 1.0 & 3.23 & 302.0 \\
\hline TMB_31 & 043911 & 290513 & $\mathrm{~B} 2$ & $\mathrm{~N}$ & 1.26 & 1.5 & 74 & 113 & 1.71 & 0.8 & 0.67 & 56.5 \\
\hline TMB_32 & 043948 & 283533 & $\mathrm{C}$ & $\mathrm{N}$ & 0.70 & 2.0 & 7 & 14 & 0.27 & 0.3 & 0.26 & 7.0 \\
\hline TMB_33 & 044110 & 253113 & $\mathrm{C}$ & $\mathrm{N}$ & 0.70 & 1.0 & 10 & 10 & 0.10 & 0.7 & 0.05 & 5.0 \\
\hline TMB_34 & 044420 & 283653 & B1 & $\mathrm{N}$ & 1.26 & 2.8 & 143 & 399 & 11.08 & 0.4 & 7.95 & 199.5 \\
\hline TMB_35 & 044612 & 250733 & A & $\mathrm{N}$ & 0.62 & 1.5 & 8 & 13 & 0.19 & 0.4 & 0.15 & 6.5 \\
\hline TMB_36 & 044643 & 245913 & $\mathrm{~B} 1$ & $\mathrm{Y}$ & 0.56 & 1.8 & 12 & 21 & 0.38 & 0.3 & 0.39 & 10.5 \\
\hline TMB_37 & 044812 & 245033 & A & $\mathrm{N}$ & 0.63 & 2.3 & 8 & 18 & 0.41 & 0.3 & 0.48 & 9.0 \\
\hline
\end{tabular}

Note.

${ }^{\text {a }}$ Estimate of minimum stellar wind mass loss rate needed to drive the bubbles. 

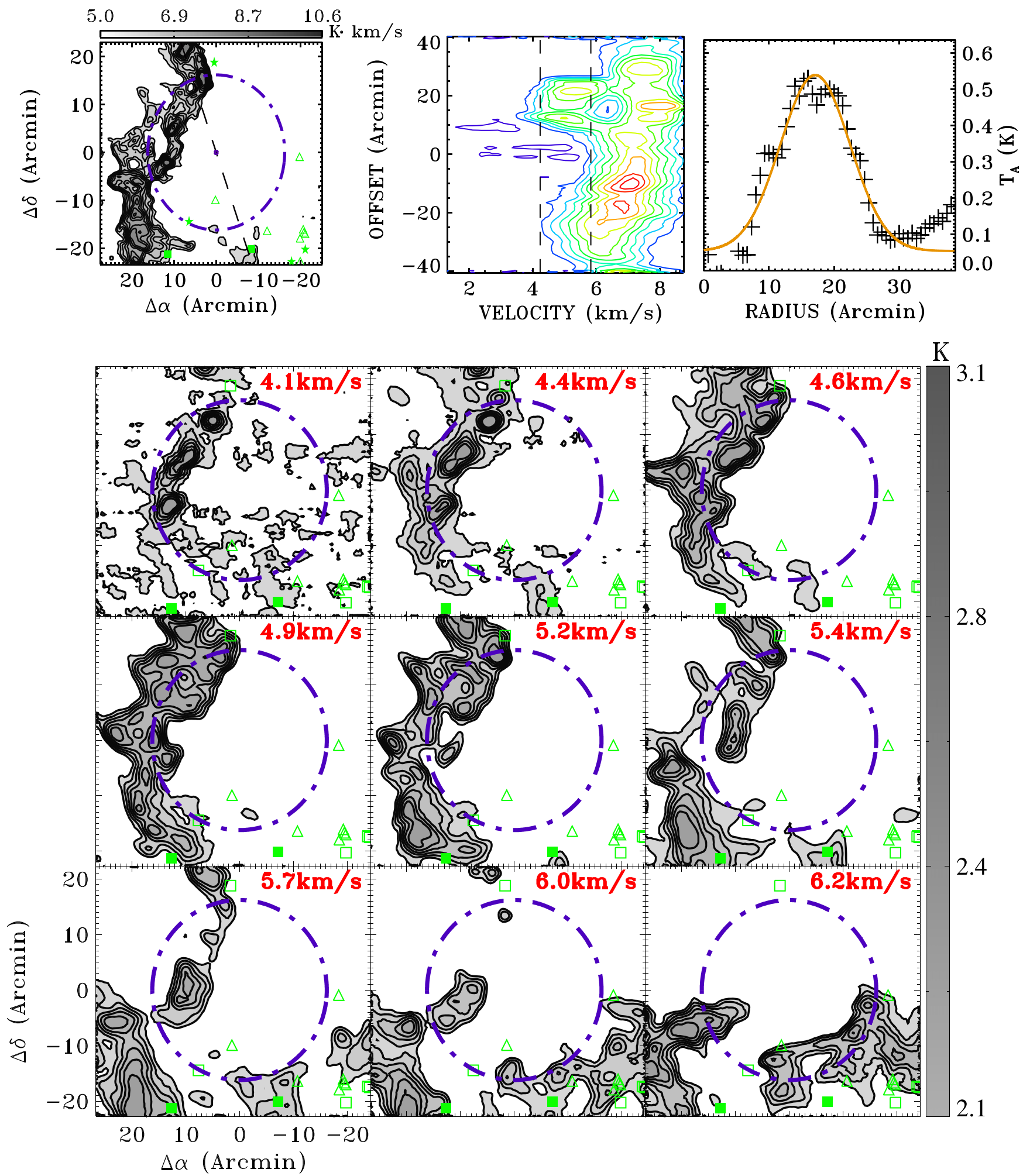

Figure 60. TMB_03. Same as Figure 58 except for the following. In the upper left panel, the integrated velocity interval is from 4.6 to $6.2 \mathrm{~km} \mathrm{~s}^{-1}$ and the contour step is $0.9 \mathrm{~K} \mathrm{~km} \mathrm{~s}^{-1}$. In the upper middle panel, the position angle is $160^{\circ}$ and the contour levels are $0.1-1.7 \mathrm{~K}$ by $0.2 \mathrm{~K}$. 

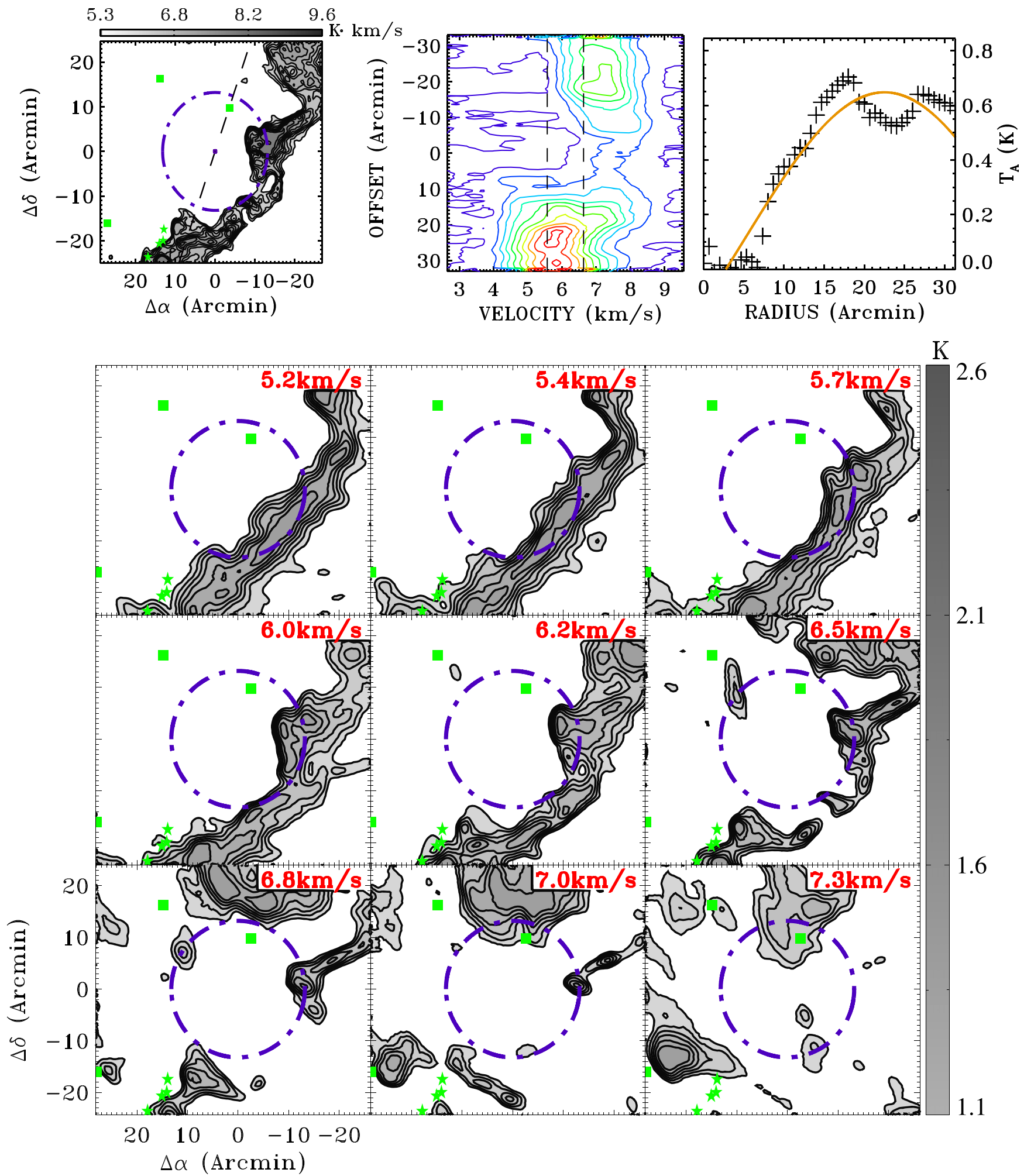

Figure 61. TMB 04. Same as Figure 58 except for the following. In the upper left panel, the integrated velocity interval is from 6.0 to $7.0 \mathrm{~km} \mathrm{~s}^{-1}$ and the contour step is $0.8 \mathrm{~K} \mathrm{~km} \mathrm{~s}^{-1}$. In the upper middle panel, the position angle is $20^{\circ}$ and the contour levels are $0.1-2.2 \mathrm{~K}$ by $0.2 \mathrm{~K}$. 

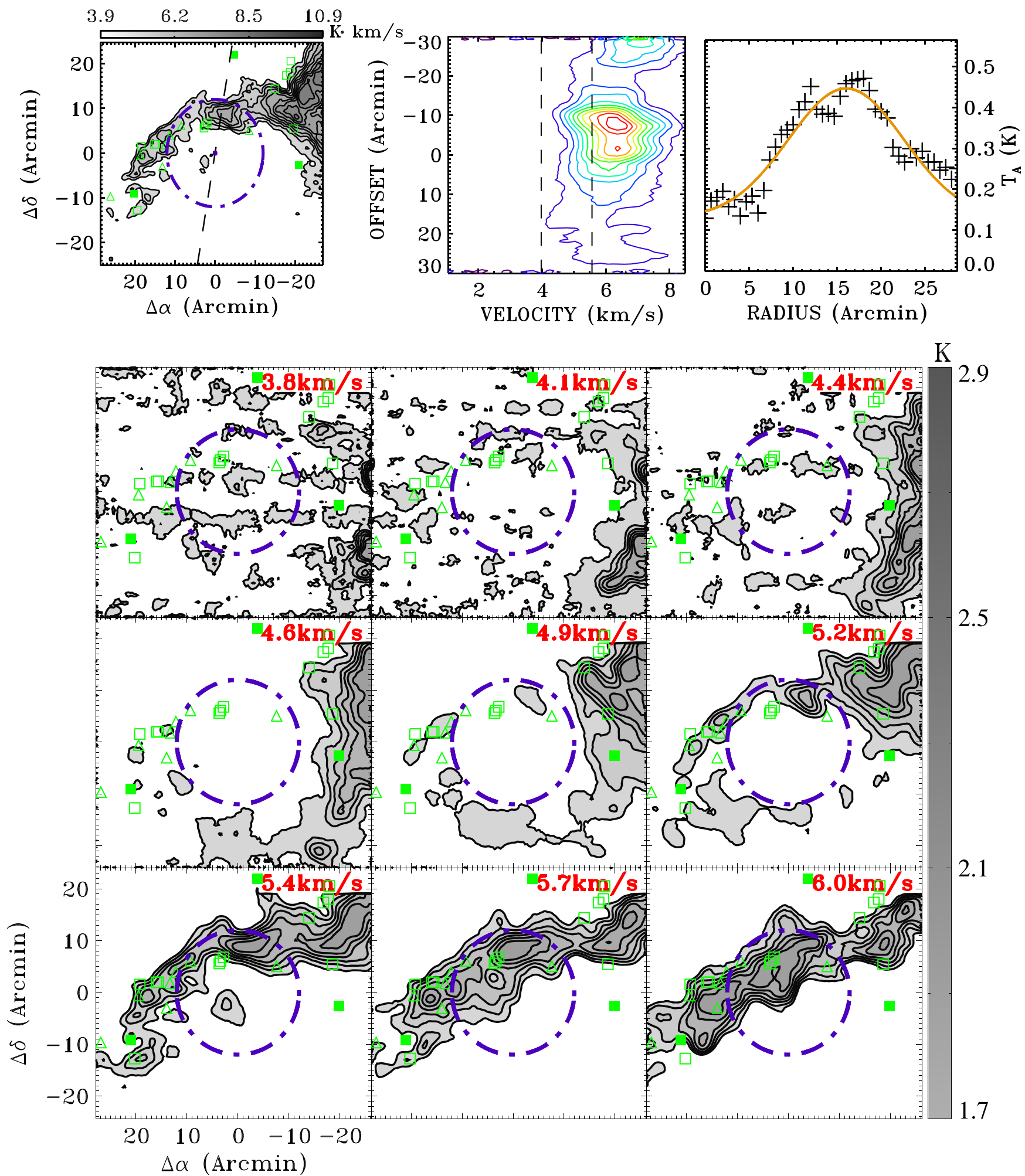

Figure 62. TMB 05. Same as Figure 58 except for the following. In the upper left panel, the integrated velocity interval is from 4.4 to $6.0 \mathrm{~km} \mathrm{~s}{ }^{-1}$, the starting contour and the contour step is 2.7 and $1 \mathrm{~K} \mathrm{~km} \mathrm{~s}^{-1}$, respectively. In the upper middle panel, the position angle is $10^{\circ}$ and the contour levels are $0.1-1.9 \mathrm{~K}$ by $0.2 \mathrm{~K}$. 

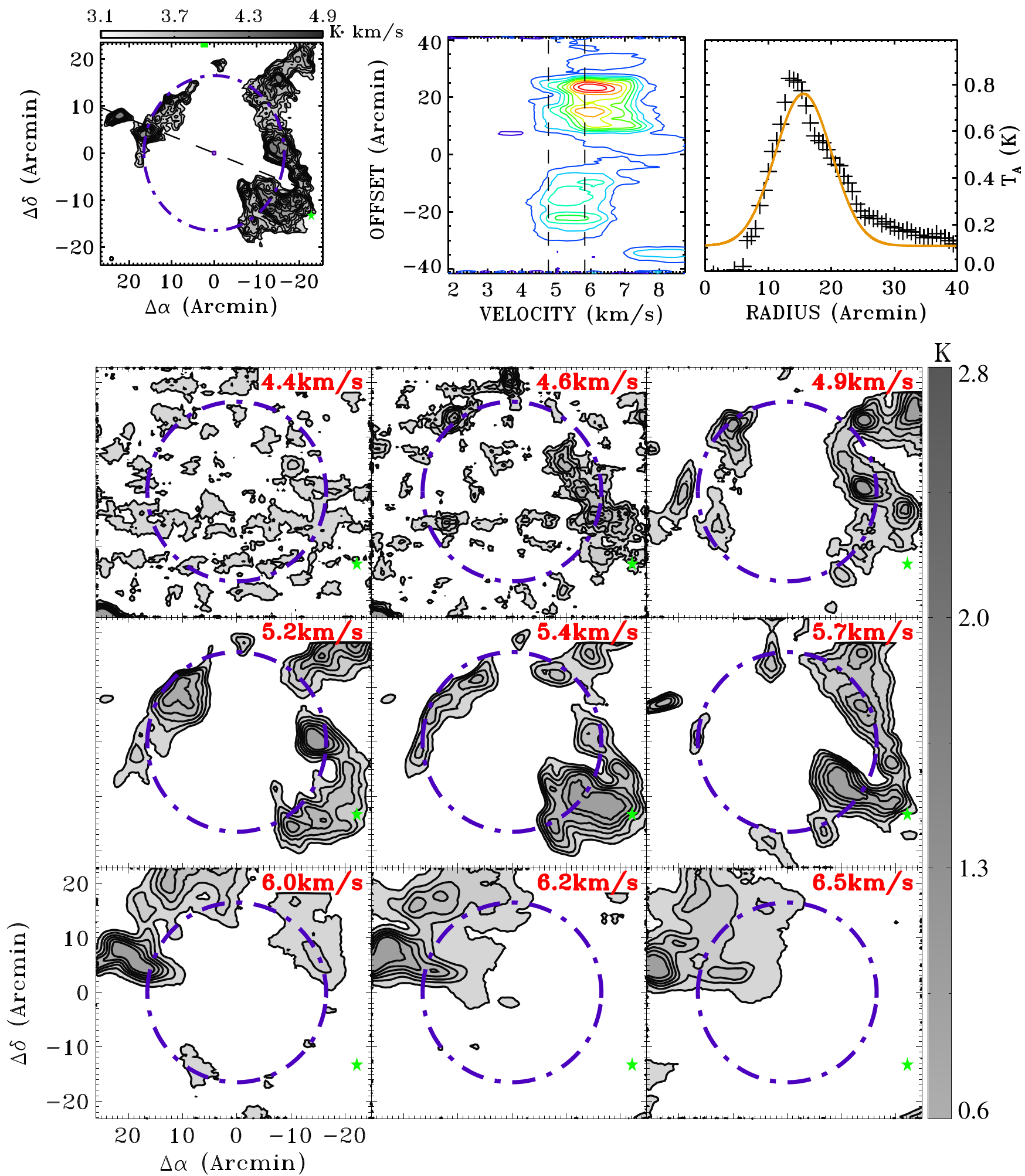

Figure 63. TMB 06. Same as Figure 58 except for the following. In the upper left panel, the integrated velocity interval is from 5.2 to $6.2 \mathrm{~km} \mathrm{~s}^{-1}$, the starting contour and the contour step are 2.1 and $0.3 \mathrm{~K} \mathrm{~km} \mathrm{~s}^{-1}$, respectively. 

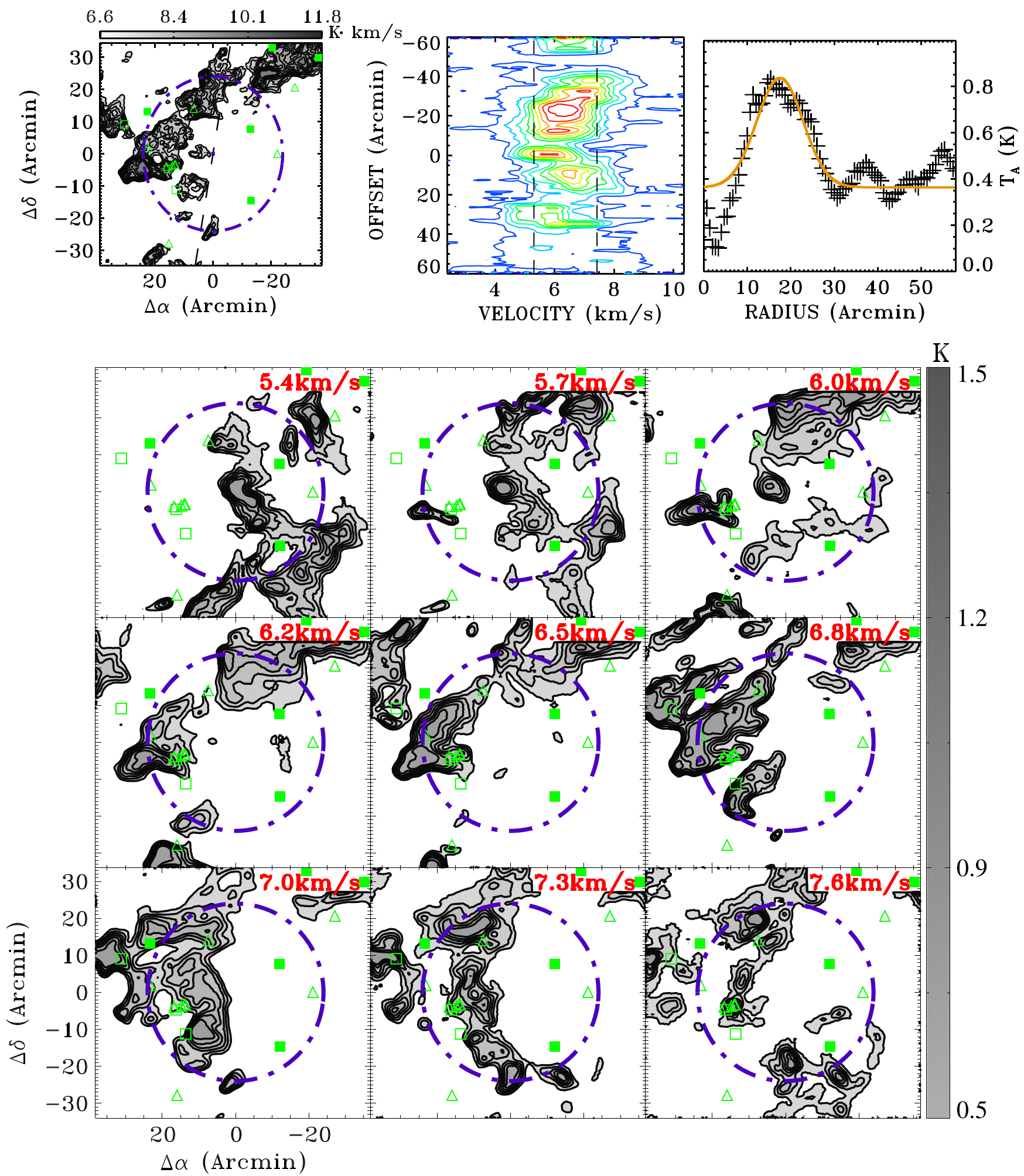

Figure 64. TMB 07. Same as Figure 58 except for the following. In the upper left panel, the integrated velocity interval is from 5.7 to $7.8 \mathrm{~km} \mathrm{~s}^{-1}$, the starting contour and the contour step are 4.7 and $0.9 \mathrm{~K} \mathrm{~km} \mathrm{~s}^{-1}$, respectively. In the upper middle panel, the position angle is $10^{\circ}$ and the contour levels are $0.1-1.1 \mathrm{~K}$ by $0.1 \mathrm{~K}$. 

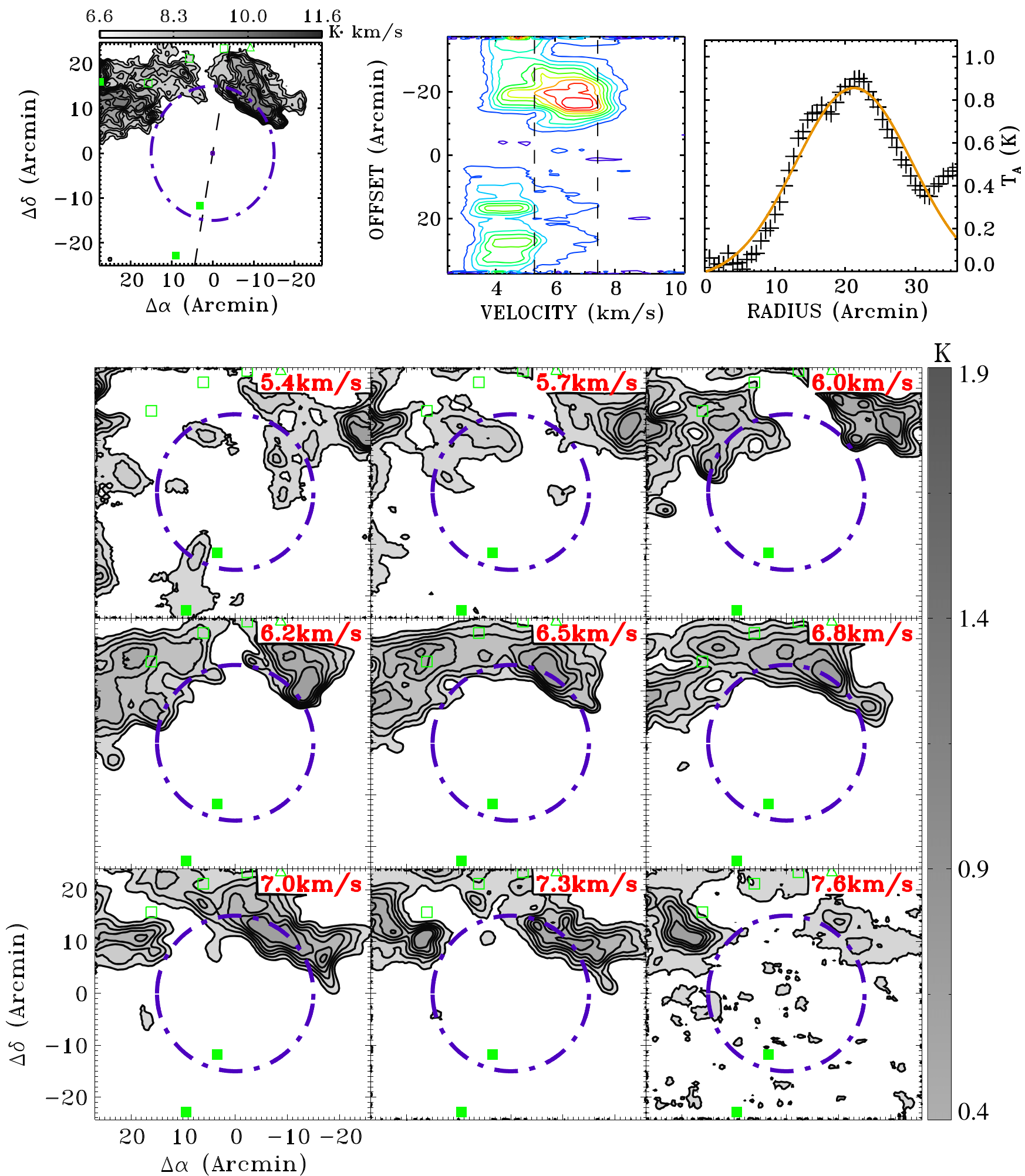

Figure 65. TMB 08. Same as Figure 58 except for the following. In the upper left panel, the integrated velocity interval is from 5.7 to $7.8 \mathrm{~km} \mathrm{~s}^{-1}$ and the contour step is $1 \mathrm{~K} \mathrm{~km} \mathrm{~s}^{-1}$. In the upper middle panel, the position angle is $10^{\circ}$ and the contour levels are $0.1-1.1 \mathrm{~K}$ by $0.1 \mathrm{~K}$. 

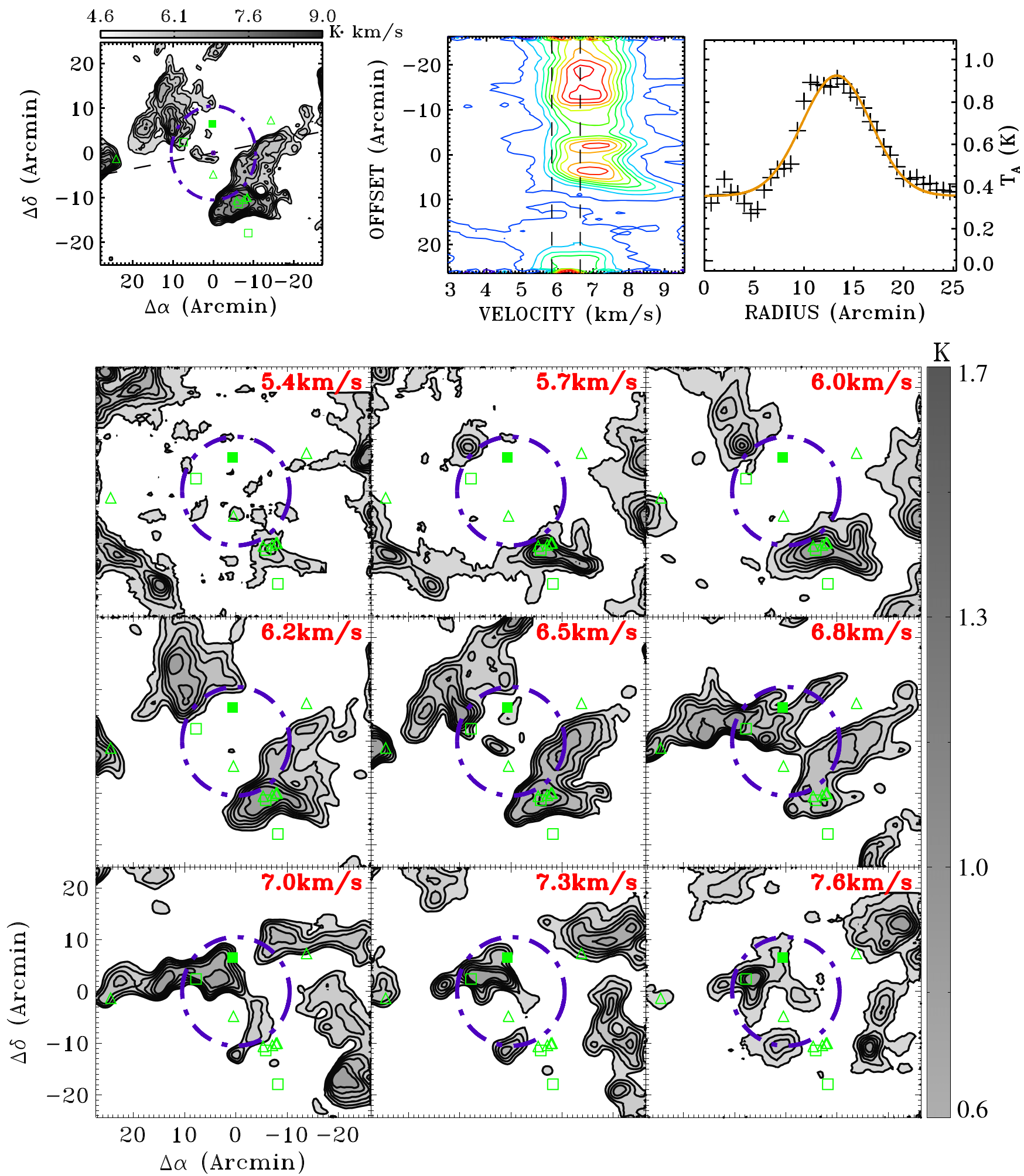

Figure 66. TMB 09. Same as Figure 58 except for the following. In the upper left panel, the integrated velocity interval is from 6.2 to $7.0 \mathrm{~km} \mathrm{~s}^{-1}$, the starting contour and the contour step are 3.4 and $0.7 \mathrm{~K} \mathrm{~km} \mathrm{~s}^{-1}$, respectively. In the upper middle panel, the position angle is $80^{\circ}$. 

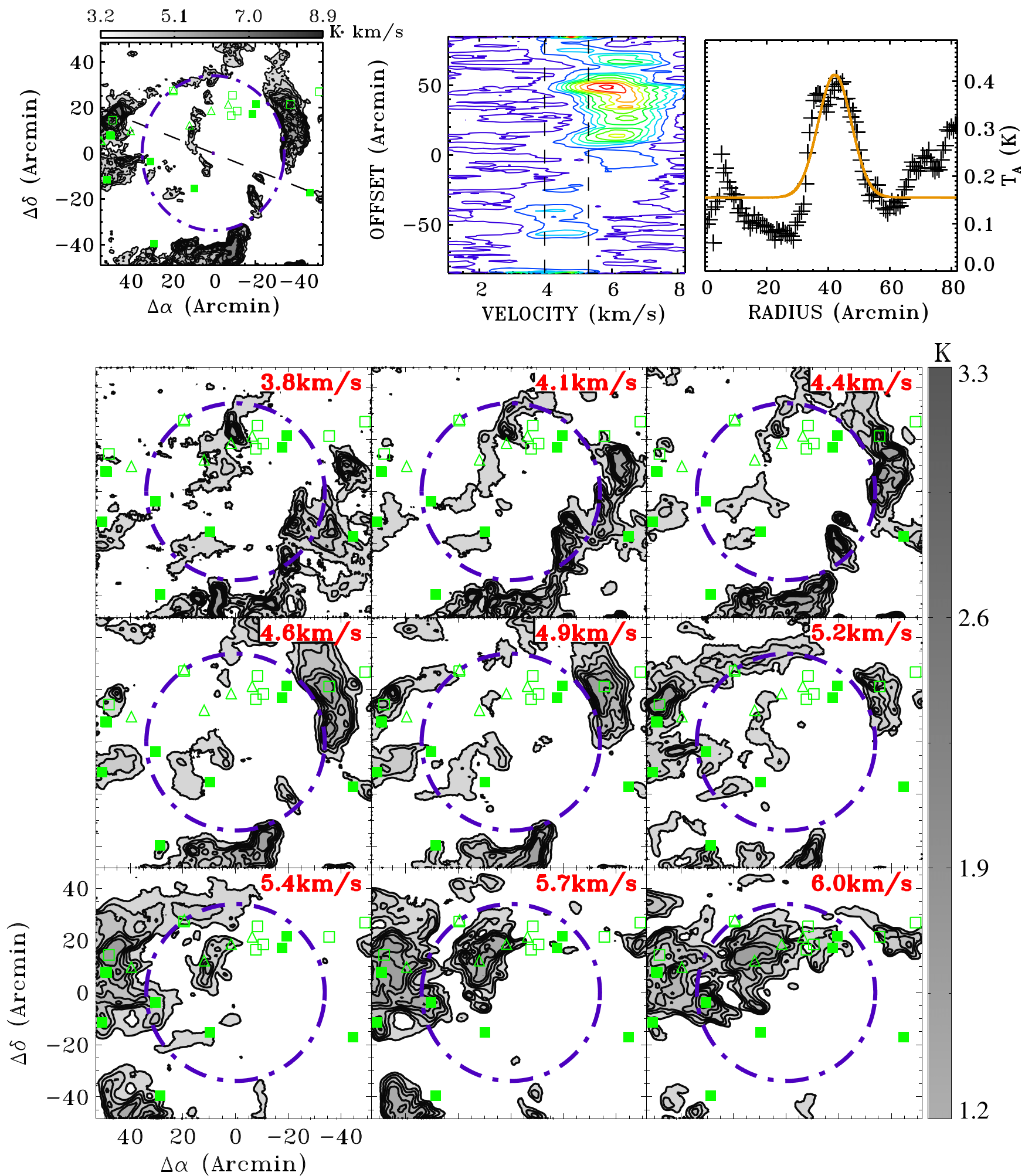

Figure 67. TMB_10. Same as Figure 58 except for the following. In the upper left panel, the integrated velocity interval is from 4.4 to $5.7 \mathrm{~km} \mathrm{~s}^{-1}$, the starting contour and the contour step are 2.3 and $0.8 \mathrm{~K} \mathrm{~km} \mathrm{~s}^{-1}$, respectively. In the upper middle panel, the contour levels are $0.1-1.8 \mathrm{~K}$ by $0.2 \mathrm{~K}$. 

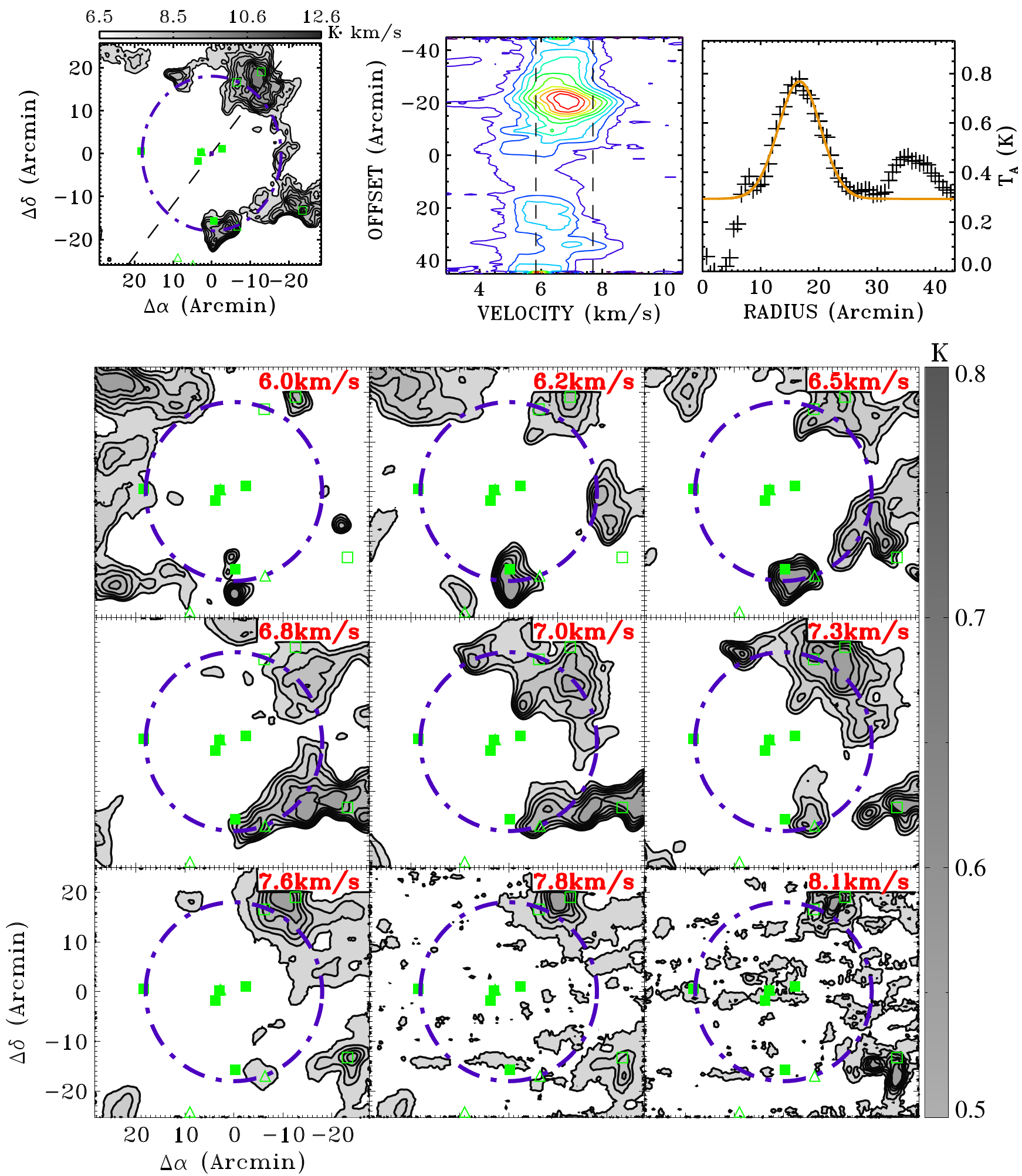

Figure 68. TMB 11. Same as Figure 58 except for the following. In the upper left panel, the integrated velocity interval is from 6.2 to $8.1 \mathrm{~km} \mathrm{~s}^{-1}$, the starting contour and the contour step are 4.8 and $0.9 \mathrm{~K} \mathrm{~km} \mathrm{~s}^{-1}$, respectively. In the upper middle panel, the position angle is $40^{\circ}$ and the contour levels are $0.1-1.6 \mathrm{~K}$ by $0.2 \mathrm{~K}$. 

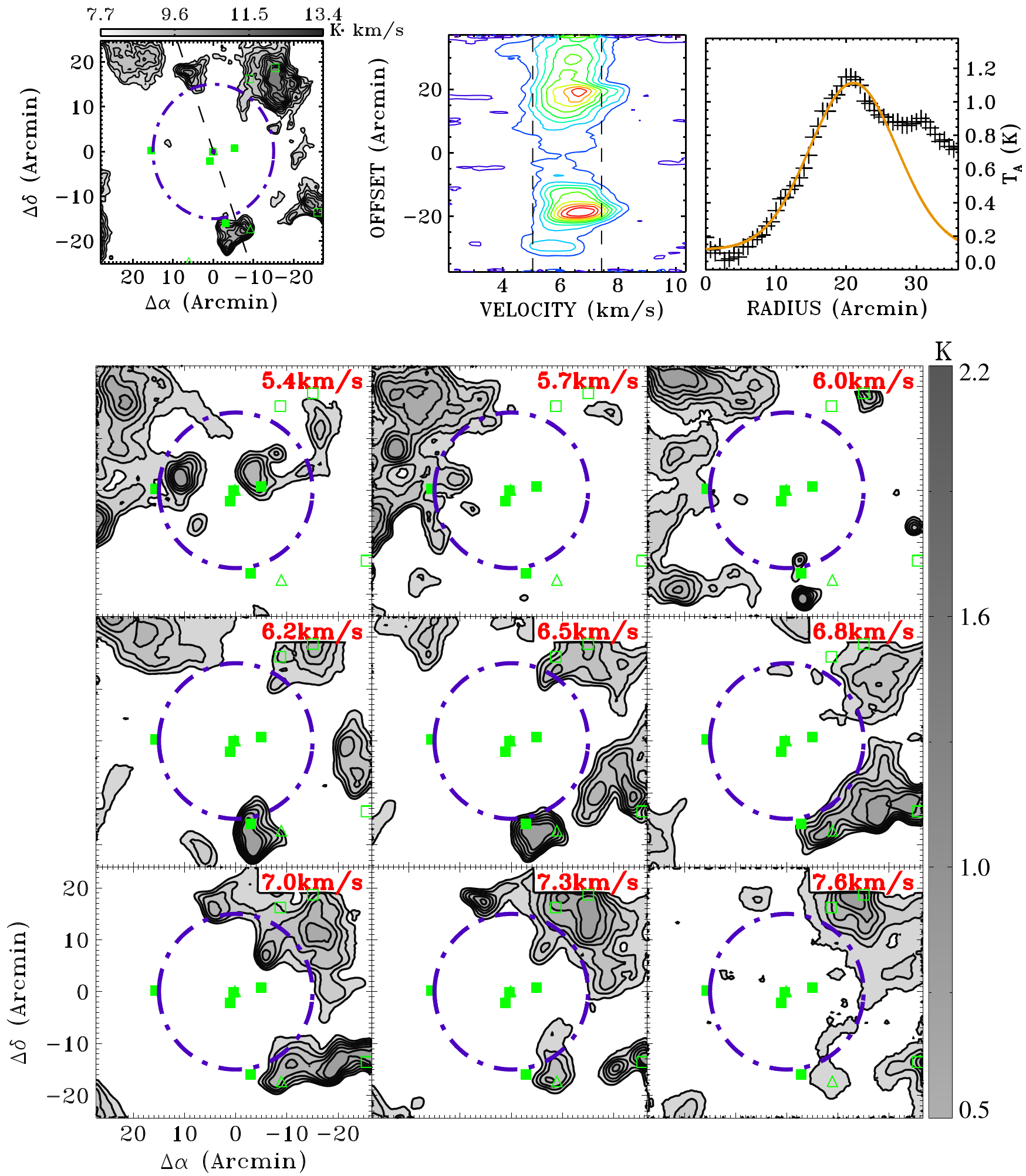

Figure 69. TMB 12. Same as Figure 58 except for the following. In the upper left panel, the integrated velocity interval is from 5.4 to $7.8 \mathrm{~km} \mathrm{~s}^{-1}$, the starting contour and the contour step are 6.2 and $0.9 \mathrm{~K} \mathrm{~km} \mathrm{~s}^{-1}$, respectively. In the upper middle panel, the position angle is $160^{\circ}$ and the contour levels are $0.1-1.5 \mathrm{~K}$ by $0.2 \mathrm{~K}$. 

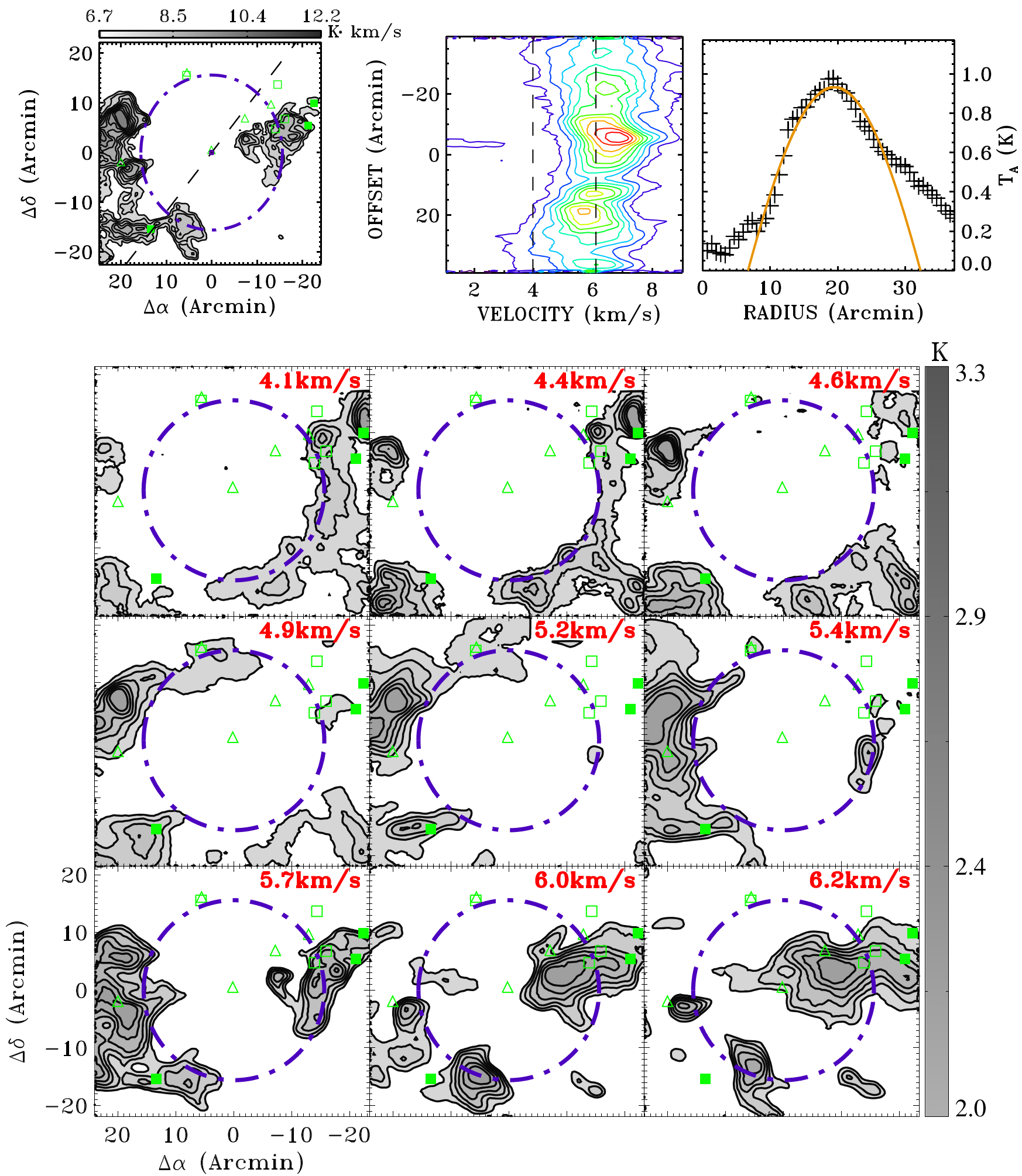

Figure 70. TMB_13. Same as Figure 58 except for the following. In the upper left panel, the integrated velocity interval is from 4.4 to $6.5 \mathrm{~km} \mathrm{~s}^{-1}$, the starting contour and the contour step are 5.0 and $0.9 \mathrm{~K} \mathrm{~km} \mathrm{~s}^{-1}$, respectively. In the upper middle panel, the position angle is $40^{\circ}$ and the contour levels are $0.1-1.7 \mathrm{~K}$ by $0.2 \mathrm{~K}$. 

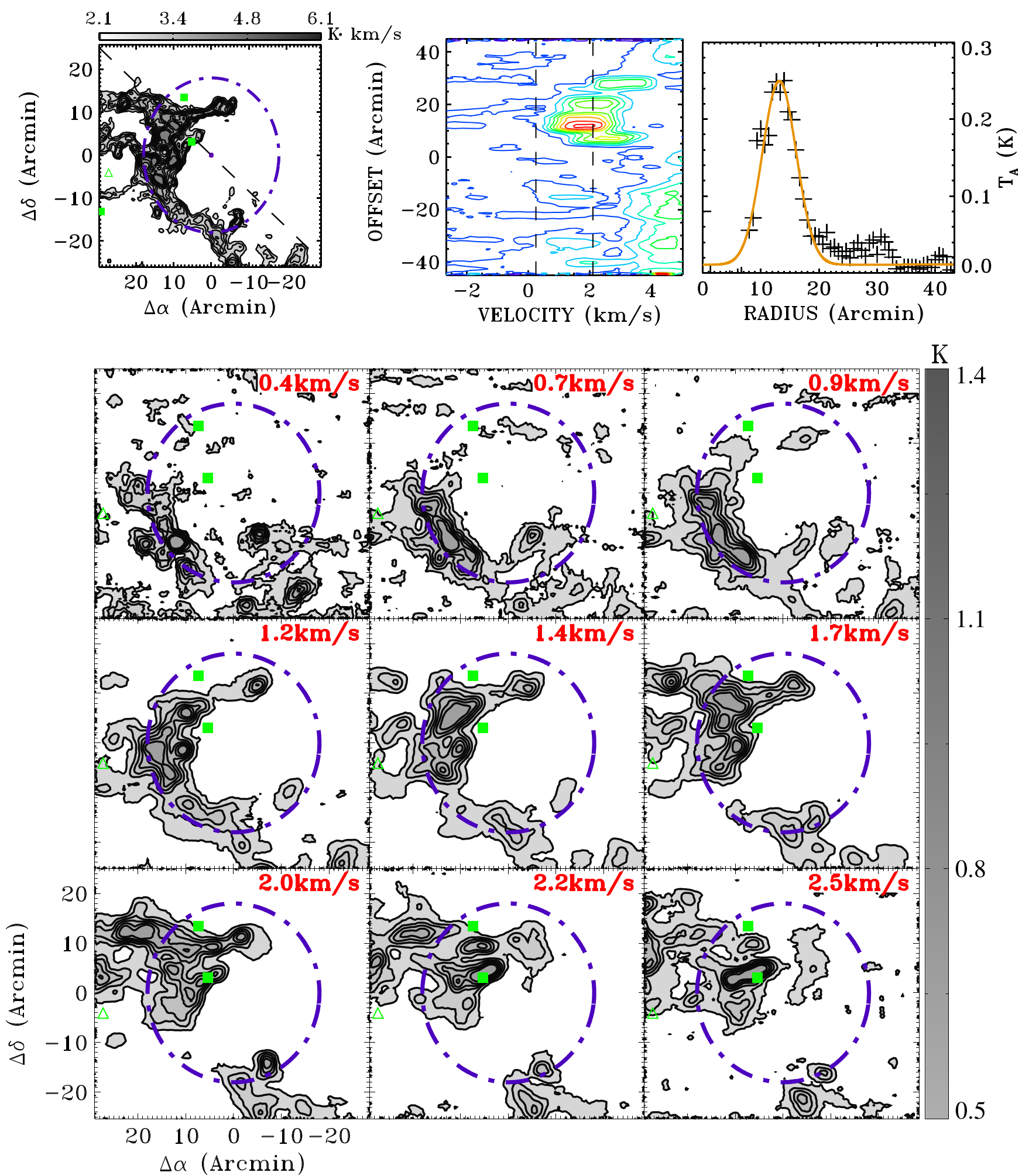

Figure 71. TMB 14. Same as Figure 58 except for the following. In the upper left panel, the integrated velocity interval is from 0.7 to $2.5 \mathrm{~km} \mathrm{~s} \mathrm{~s}^{-1}$ and the starting contour is $1.3 \mathrm{~K} \mathrm{~km} \mathrm{~s}^{-1}$. In the upper middle panel, the position angle is $130^{\circ}$ and the contour levels are $0.1-0.8 \mathrm{~K}$ by $0.1 \mathrm{~K}$. 

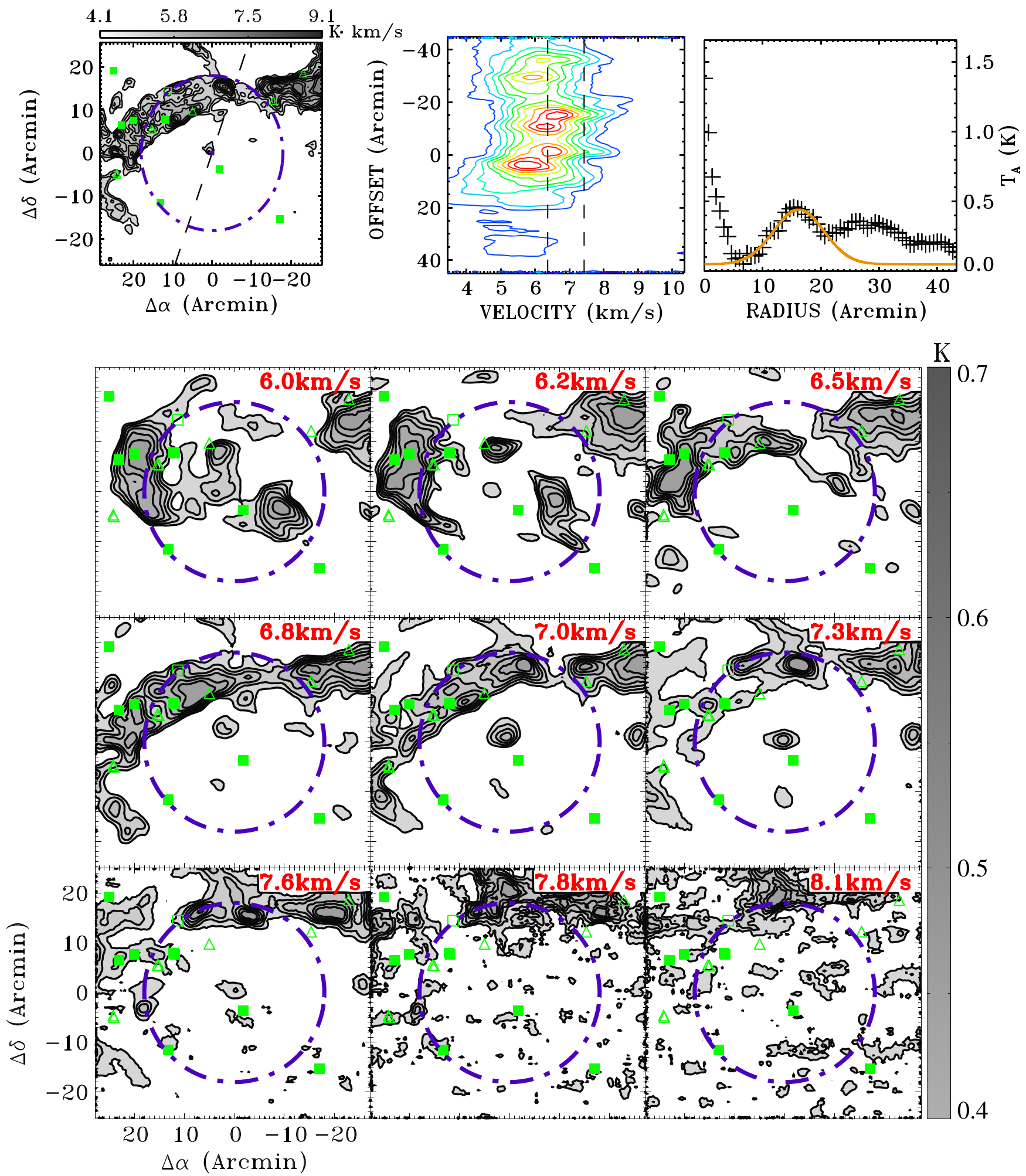

Figure 72. TMB 15. Same as Figure 58 except for the following. In the upper left panel, the integrated velocity interval is from $6.8-7.8 \mathrm{~km} \mathrm{~s}^{-1}$, the starting contour and the contour step are 2.9 and $0.8 \mathrm{~K} \mathrm{~km} \mathrm{~s}^{-1}$, respectively. In the upper middle panel, the position angle is $20^{\circ}$ and the contour levels are $0.1-1.4 \mathrm{~K}$ by $0.1 \mathrm{~K}$. 

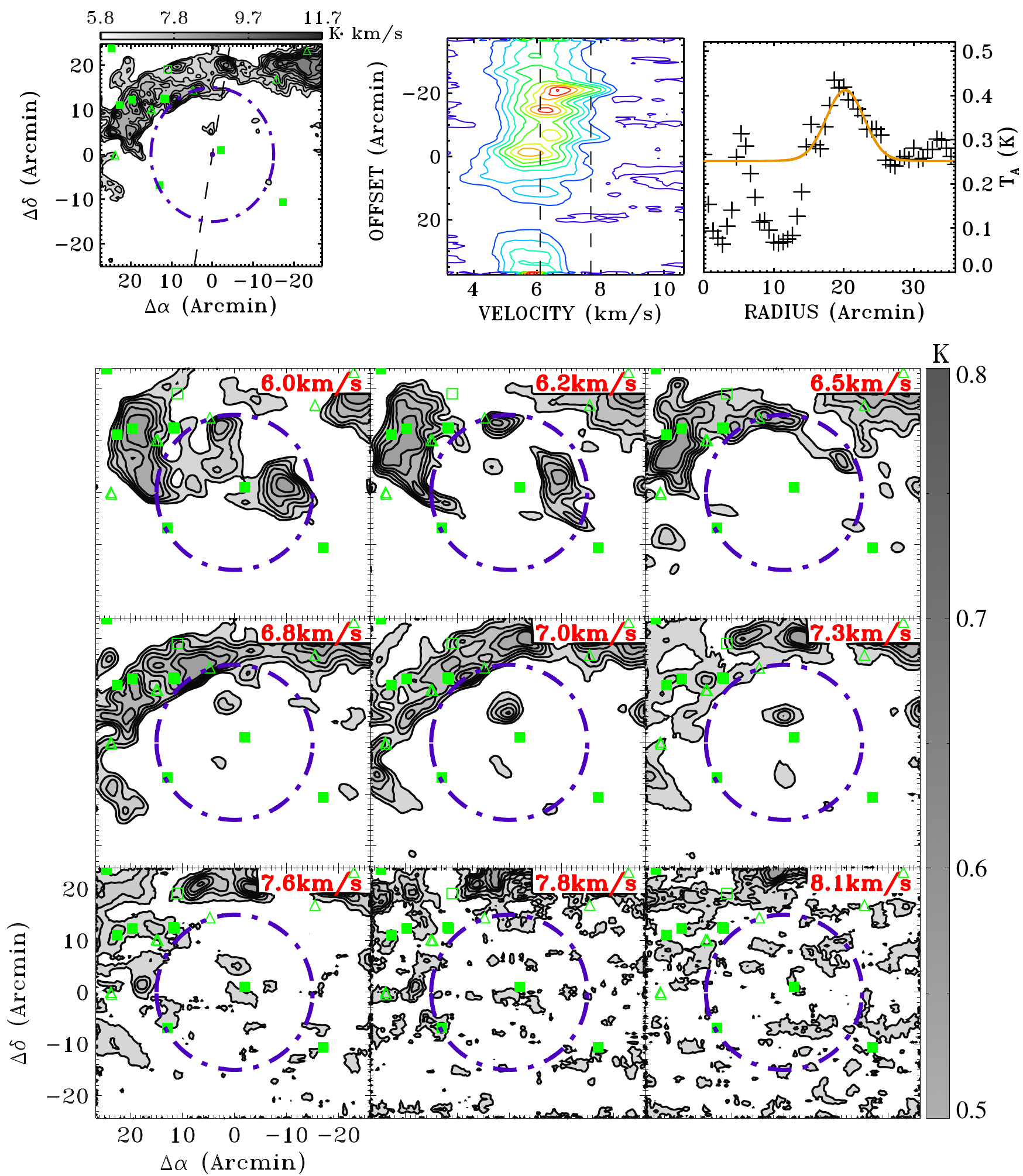

Figure 73. TMB 16. Same as Figure 58 except for the following. In the upper left panel, the integrated velocity interval is from 6.5 to $8.1 \mathrm{~km} \mathrm{~s}^{-1}$, the starting contour and the contour step are 4.2 and $0.9 \mathrm{~K} \mathrm{~km} \mathrm{~s}^{-1}$, respectively. In the upper middle panel, the position angle is $10^{\circ}$ and the contour levels are $0.1-1.8 \mathrm{~K}$ by $0.2 \mathrm{~K}$. 

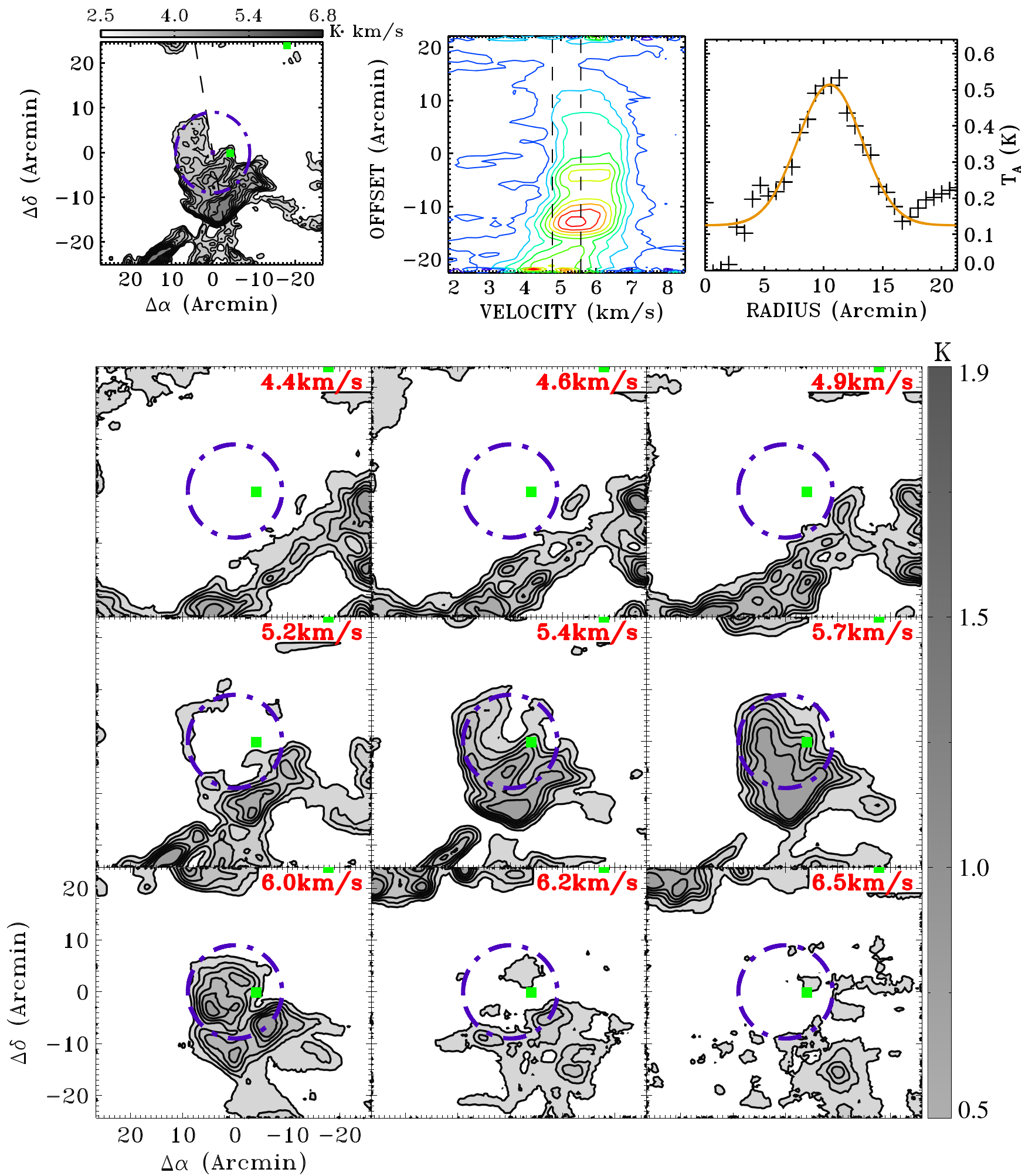

Figure 74. TMB 17. Same as Figure 58 except for the following. In the upper left panel, the integrated velocity interval is from 5.2 to $6.0 \mathrm{~km} \mathrm{~s} \mathrm{~s}^{-1}$ and the starting contour is $1.6 \mathrm{~K} \mathrm{~km} \mathrm{~s}^{-1}$. In the upper middle panel, the position angle is $170^{\circ}$ and the contour levels are $0.1-1.3 \mathrm{~K}$ by $0.1 \mathrm{~K}$. 

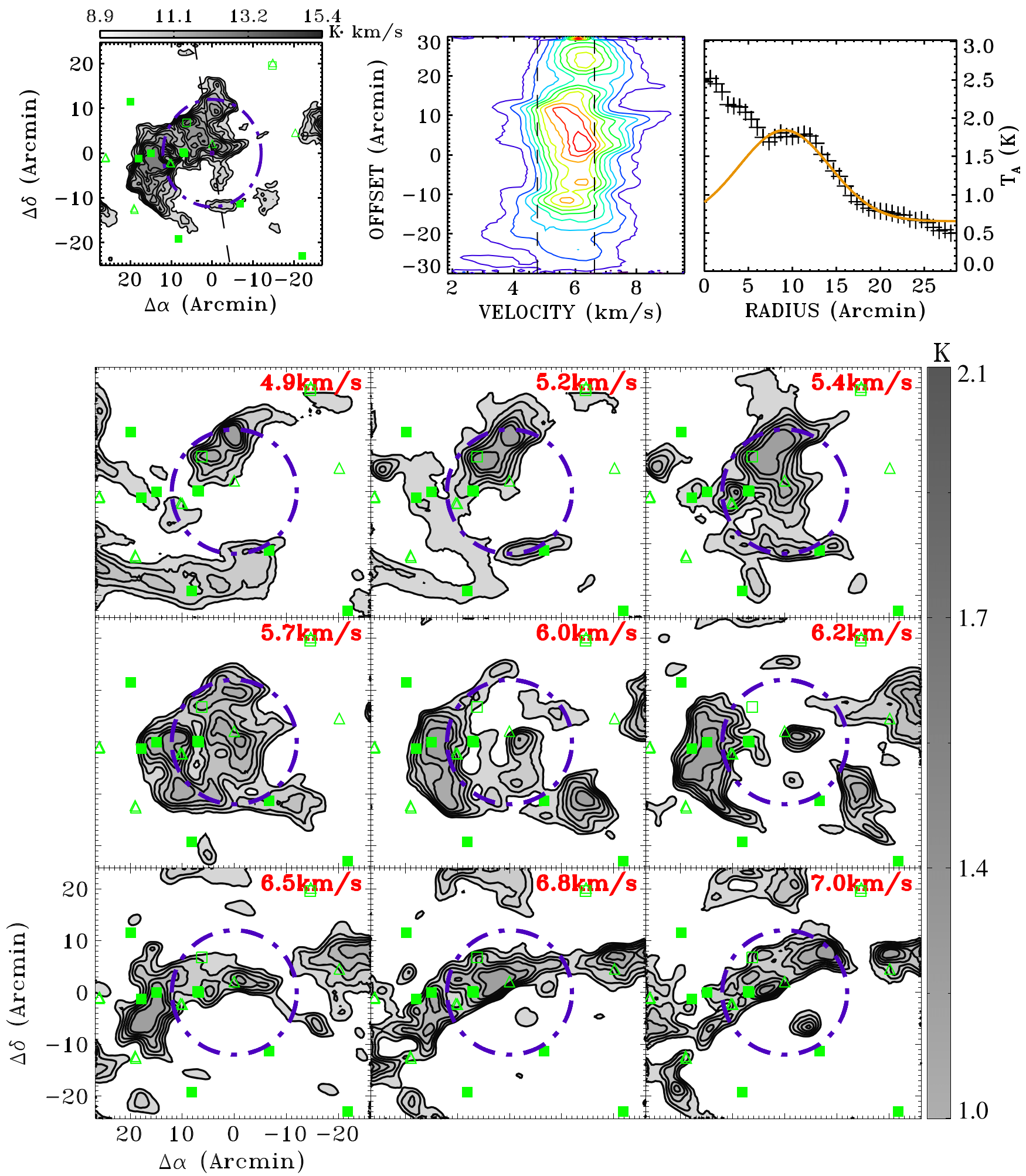

Figure 75. TMB 18. Same as Figure 58 except for the following. In the upper left panel, the integrated velocity interval is from 5.2 to $7.0 \mathrm{~km} \mathrm{~s}{ }^{-1}$, the starting contour and the contour step are 7.2 and $1 \mathrm{~K} \mathrm{~km} \mathrm{~s}^{-1}$, respectively. In the upper middle panel, the position angle is $170^{\circ}$ and the contour levels are $0.1-1.9 \mathrm{~K}$ by $0.2 \mathrm{~K}$. 

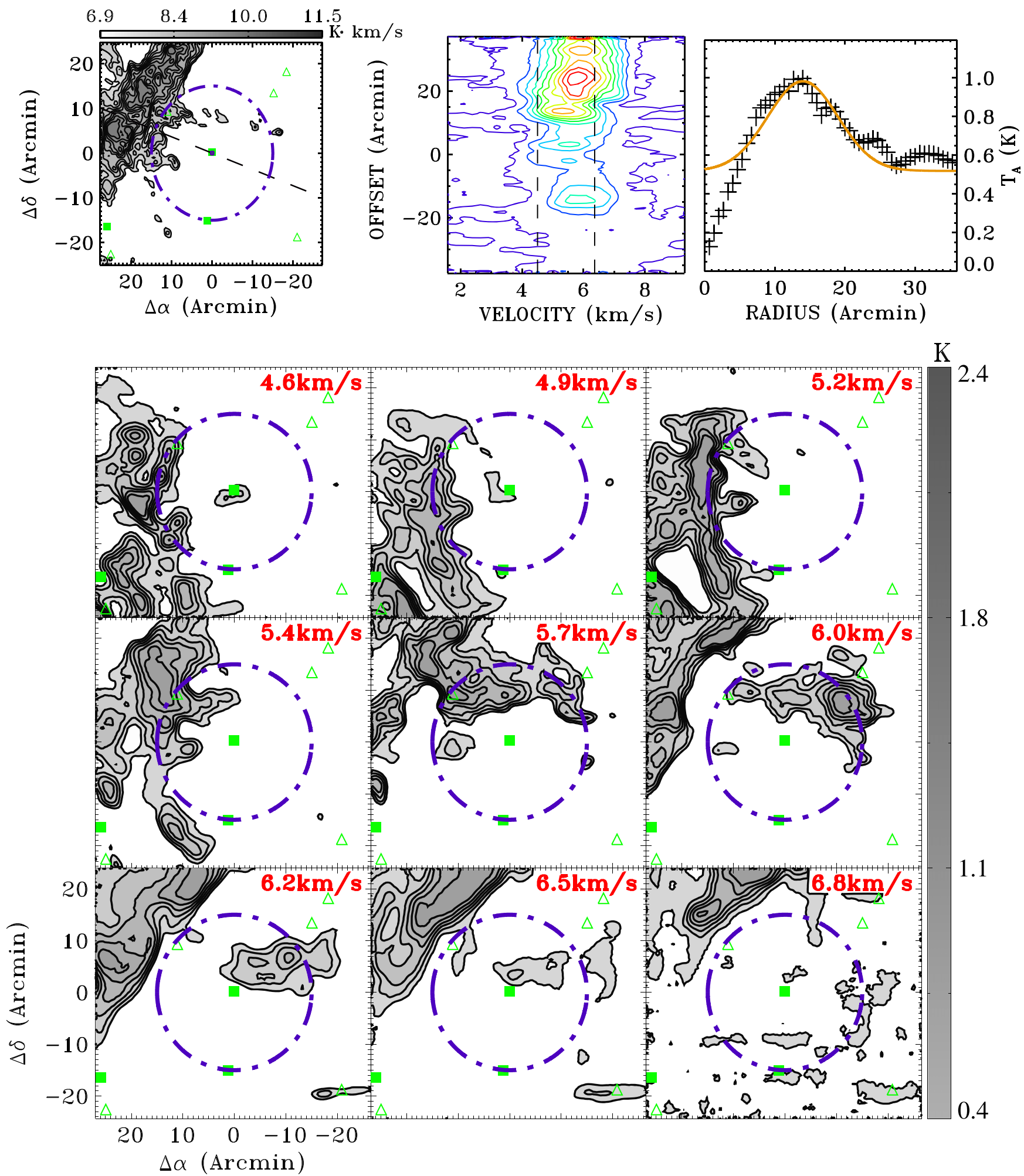

Figure 76. TMB 19. Same as Figure 58 except for the following. In the upper left panel, the integrated velocity interval is from 4.9 to $6.8 \mathrm{~km} \mathrm{~s}^{-1}$, the starting contour and the contour step are 5.0 and $0.8 \mathrm{~K} \mathrm{~km} \mathrm{~s}^{-1}$, respectively. In the upper middle panel, the contour levels are $0.1-1.5 \mathrm{~K}$ by $0.1 \mathrm{~K}$. 

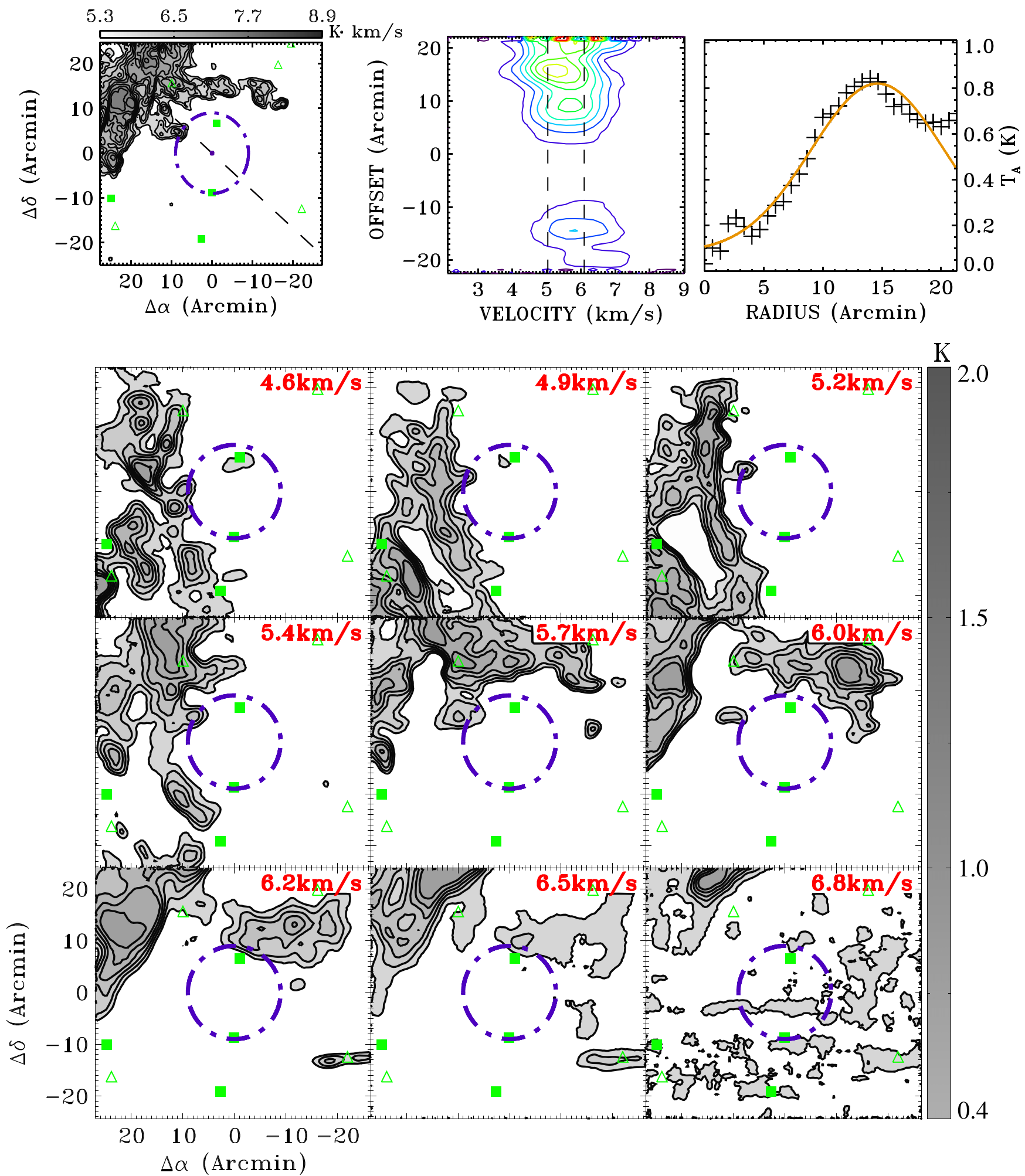

Figure 77. TMB 20. Same as Figure 58 except for the following. In the upper left panel, the integrated velocity interval is from 5.4 to $6.5 \mathrm{~km} \mathrm{~s} \mathrm{~s}^{-1}$ and the starting contour is $3.6 \mathrm{~K} \mathrm{~km} \mathrm{~s}^{-1}$. In the upper middle panel, the position angle is $130^{\circ}$ and the contour levels are $0.1-1.8 \mathrm{~K}$ by $0.2 \mathrm{~K}$. 

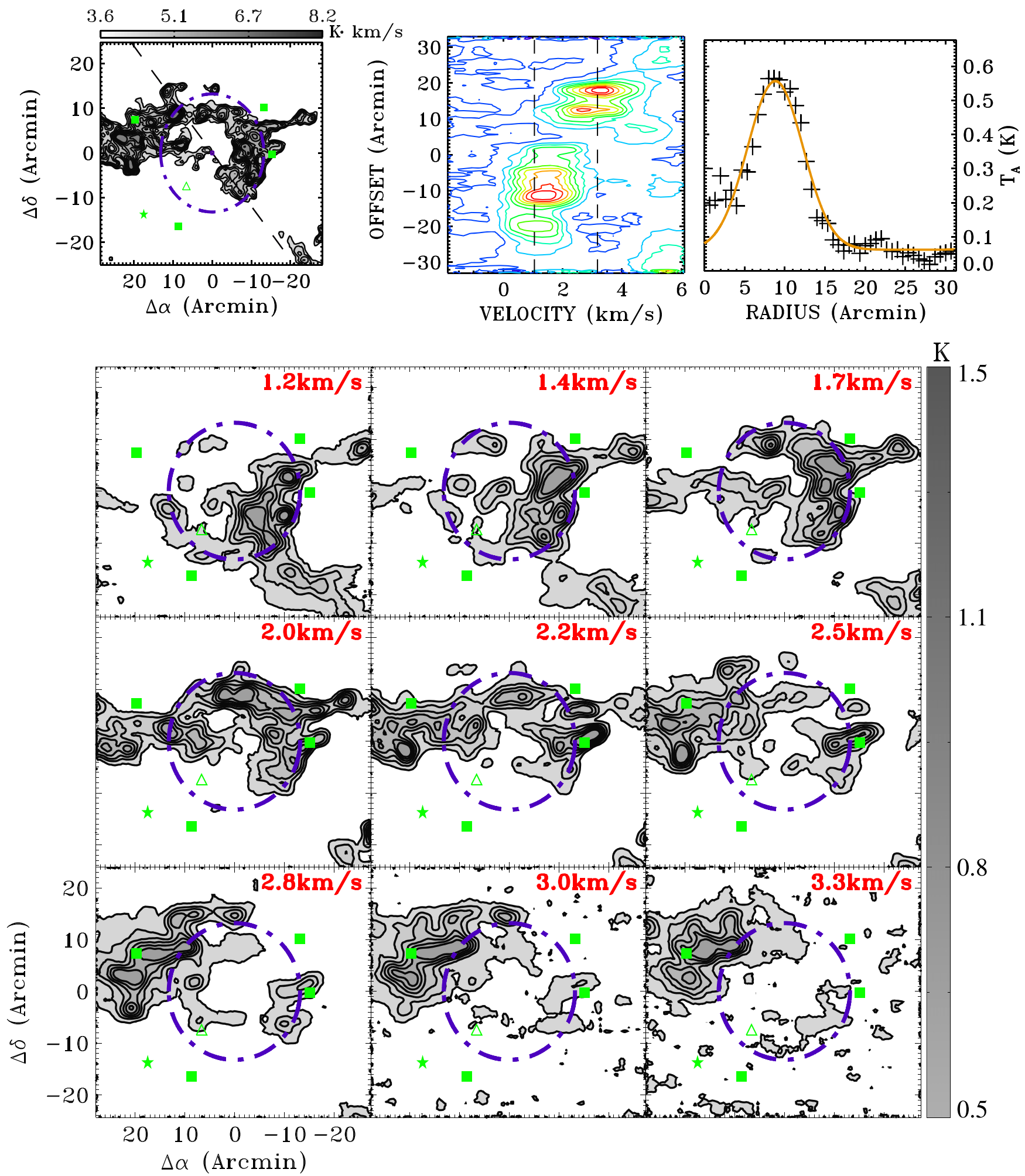

Figure 78. TMB 21. Same as Figure 58 except for the following. In the upper left panel, the integrated velocity interval is from 1.4 to $3.6 \mathrm{~km} \mathrm{~s}^{-1}$, the starting contour and the contour step are 2.2 and $0.7 \mathrm{~K} \mathrm{~km} \mathrm{~s}^{-1}$, respectively. In the upper middle panel, the position angle is $140^{\circ}$ and the contour levels are $0.1-0.8 \mathrm{~K}$ by $0.1 \mathrm{~K}$. 

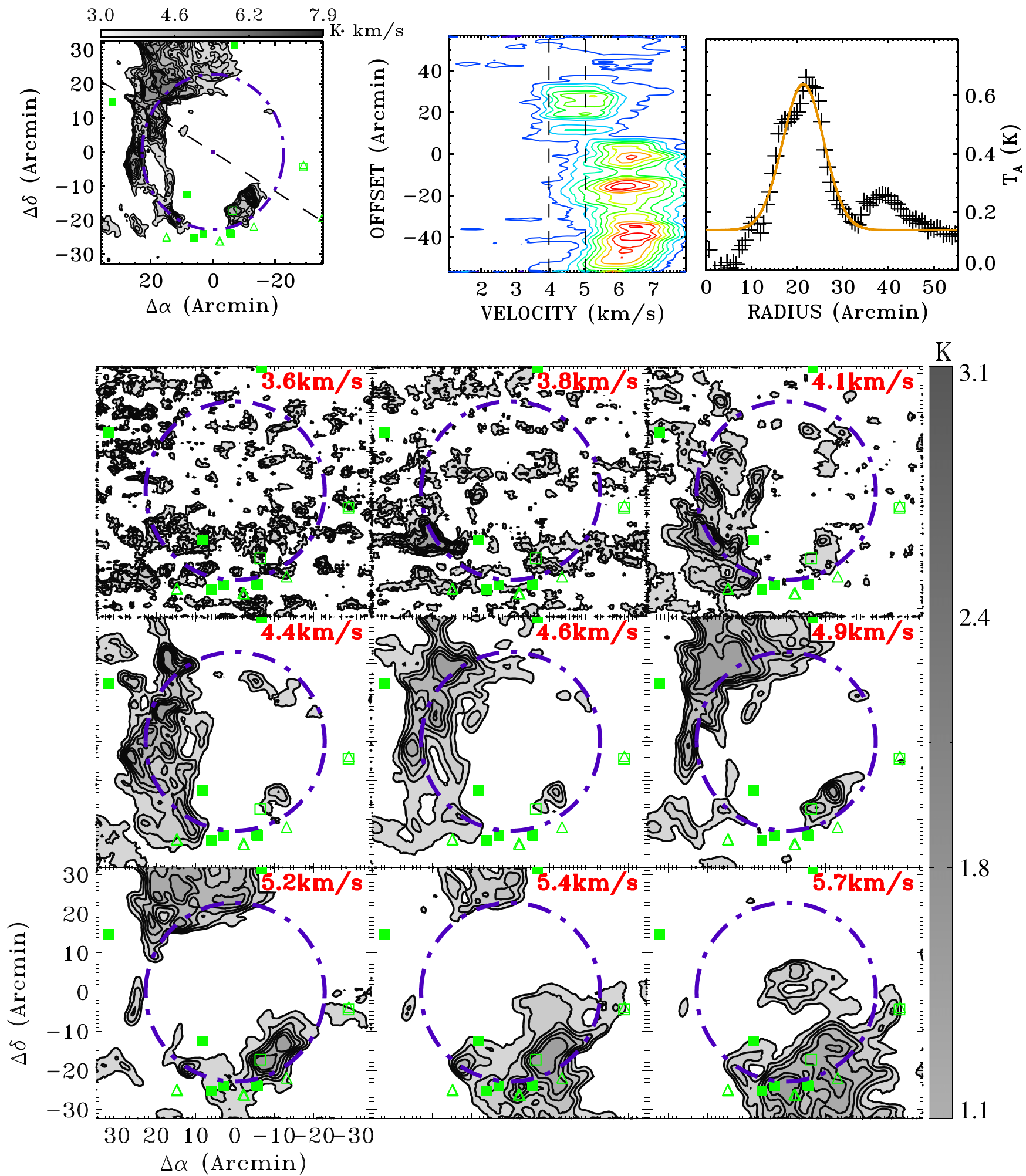

Figure 79. TMB 22. Same as Figure 58 except for the following. In the upper left panel, the integrated velocity interval is from 4.4 to $5.4 \mathrm{~km} \mathrm{~s}^{-1}$, the starting contour and the contour step are 2.0 and $0.7 \mathrm{~K} \mathrm{~km} \mathrm{~s}^{-1}$, respectively. In the upper middle panel, the position angle is $120^{\circ}$ and the contour levels are $0.1-1.5 \mathrm{~K}$ by $0.1 \mathrm{~K}$. 

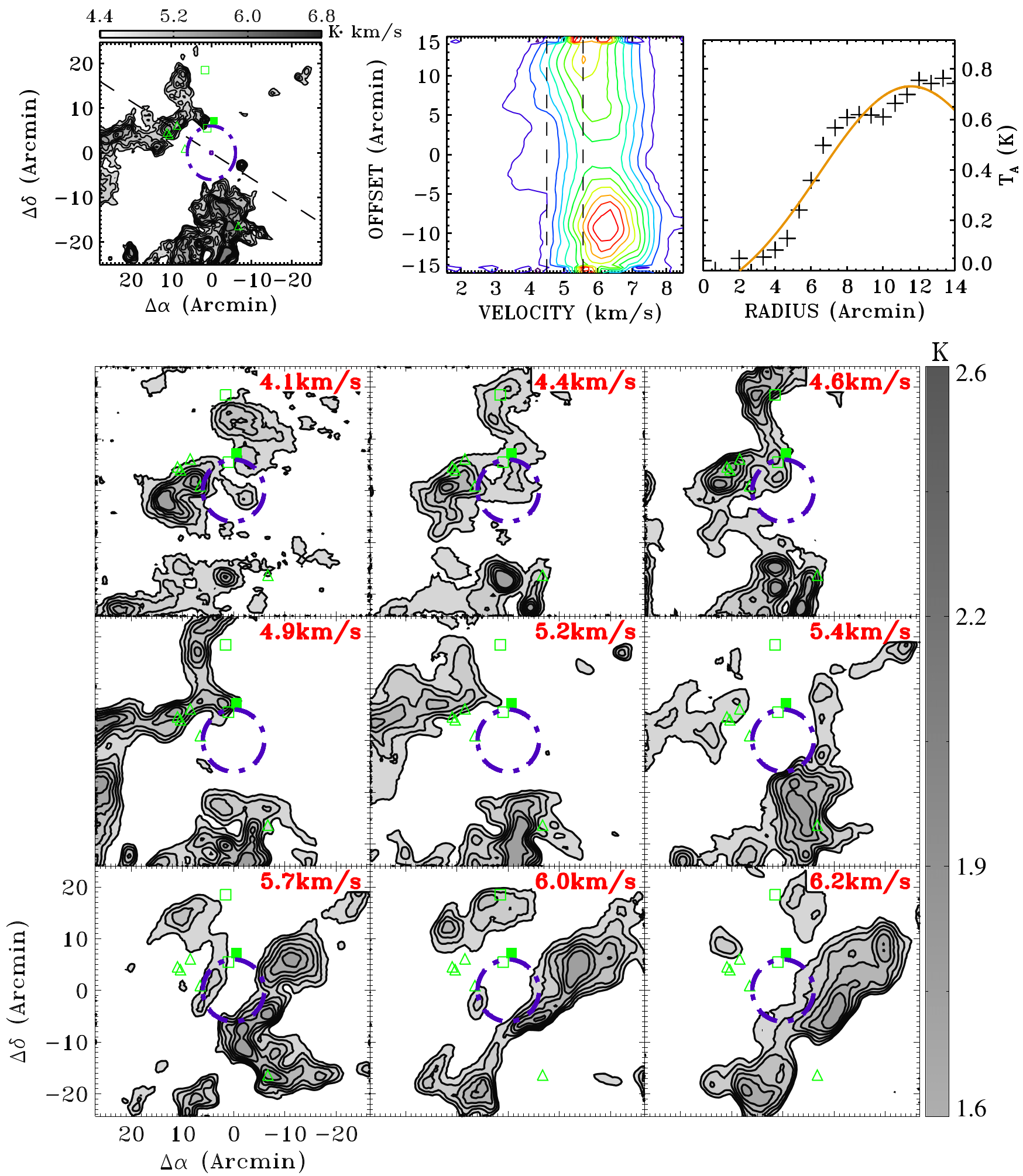

Figure 80. TMB_23. Same as Figure 58 except for the following. In the upper left panel, the integrated velocity interval is from 4.9 to $6.0 \mathrm{~km} \mathrm{~s}^{-1}$, the starting contour and the contour step are 3.6 and $0.4 \mathrm{~K} \mathrm{~km} \mathrm{~s}^{-1}$, respectively. In the upper middle panel, the position angle is $120^{\circ}$ and the contour levels are $0.1-1.7 \mathrm{~K}$ by $0.2 \mathrm{~K}$. 

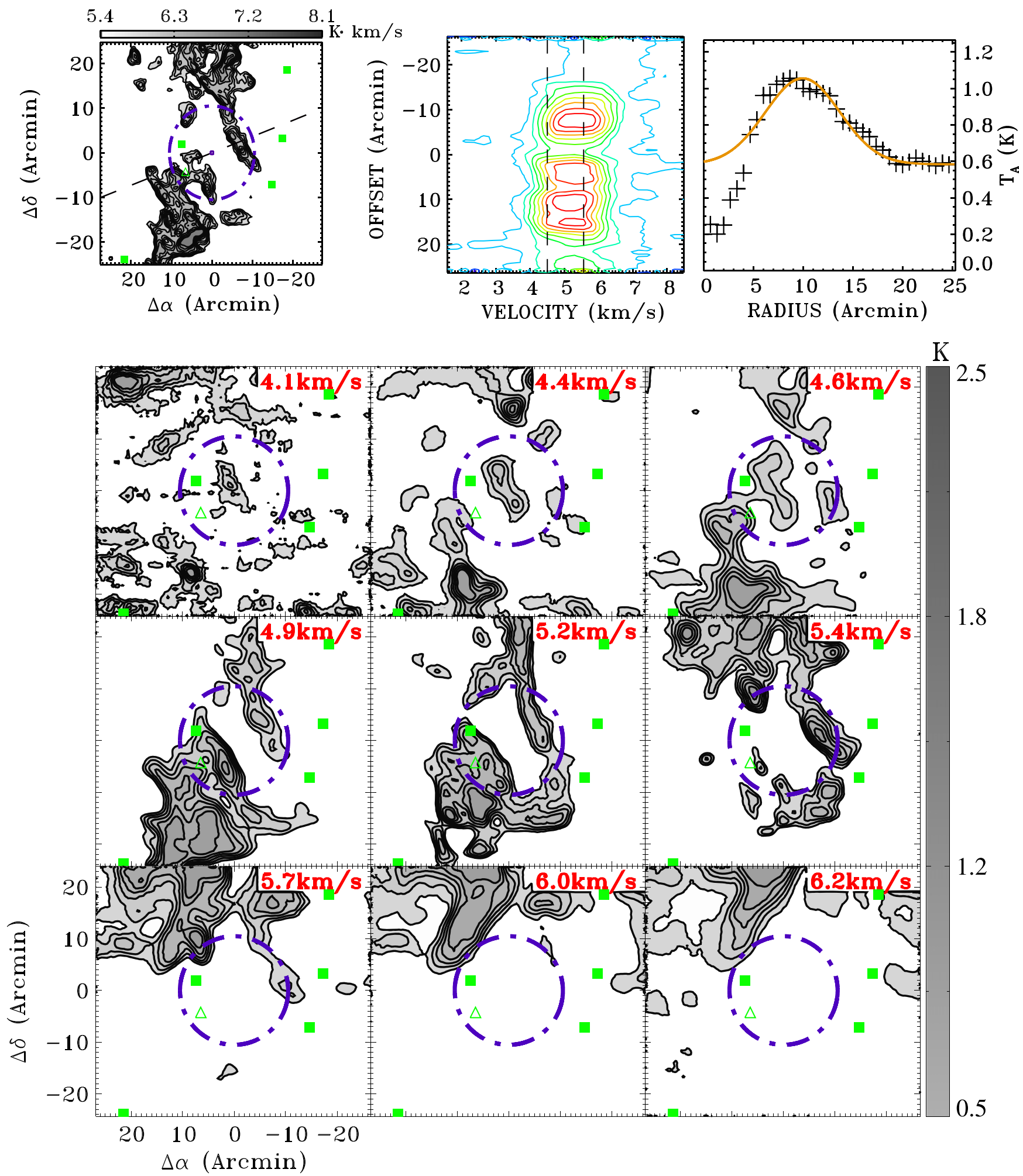

Figure 81. TMB_24. Same as Figure 58 except for the following. In the upper left panel, the integrated velocity interval is from 4.9 to $6.0 \mathrm{~km} \mathrm{~s}^{-1}$, the starting contour and the contour step are 4.2 and $0.5 \mathrm{~K} \mathrm{~km} \mathrm{~s}^{-1}$, respectively. In the upper middle panel, the position angle is $70^{\circ}$ and the contour levels are $0.1-0.9 \mathrm{~K}$ by $0.1 \mathrm{~K}$. 

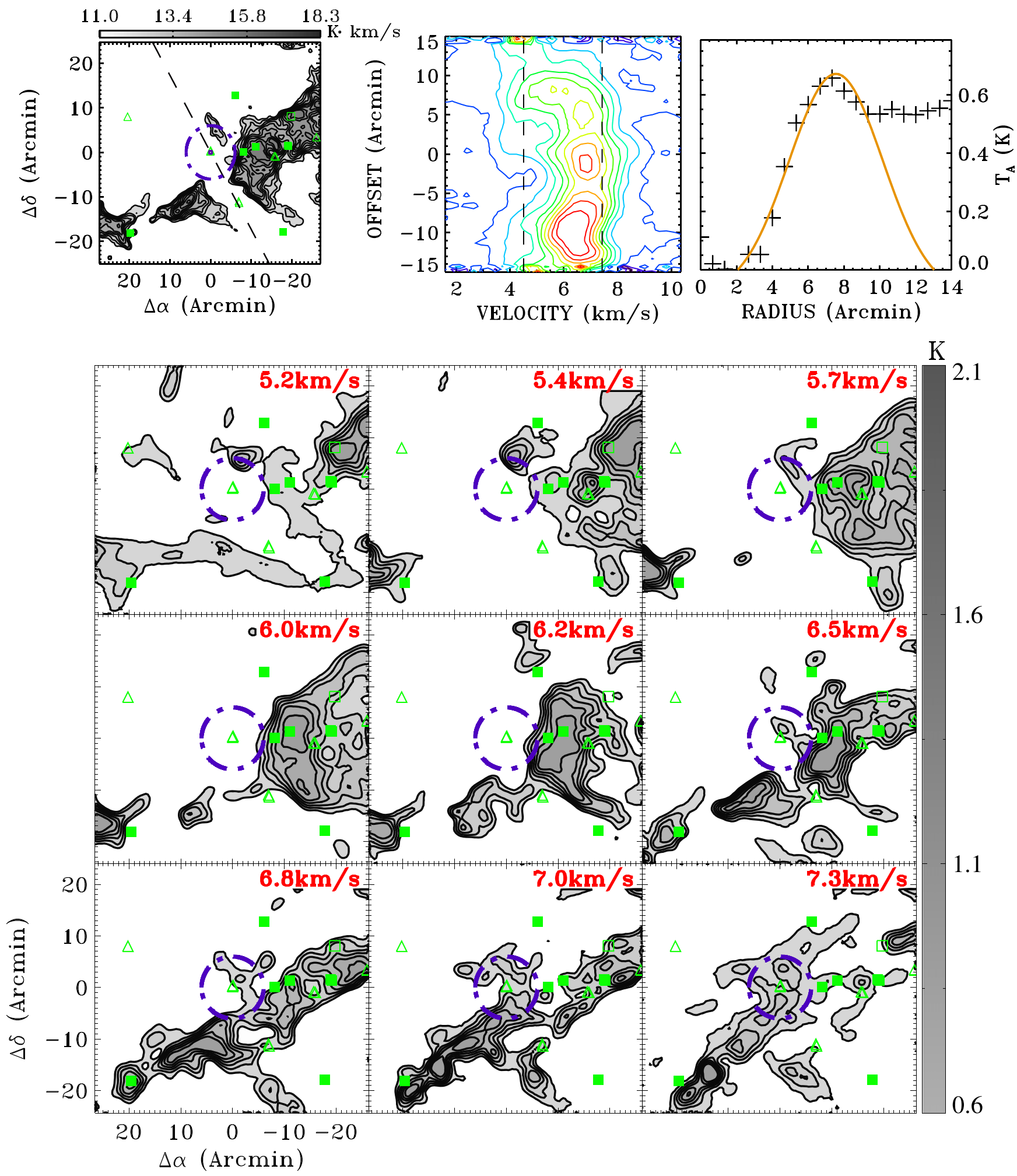

Figure 82. TMB_25. Same as Figure 58 except for the following. In the upper left panel, the integrated velocity interval is from 4.9 to $7.8 \mathrm{~km} \mathrm{~s}^{-1}$, the starting contour and the contour step are 8.2 and $1.2 \mathrm{~K} \mathrm{~km} \mathrm{~s}^{-1}$, respectively. In the upper middle panel, the position angle is $150^{\circ}$ and the contour levels are $0.1-1.0 \mathrm{~K}$ by $0.1 \mathrm{~K}$. 

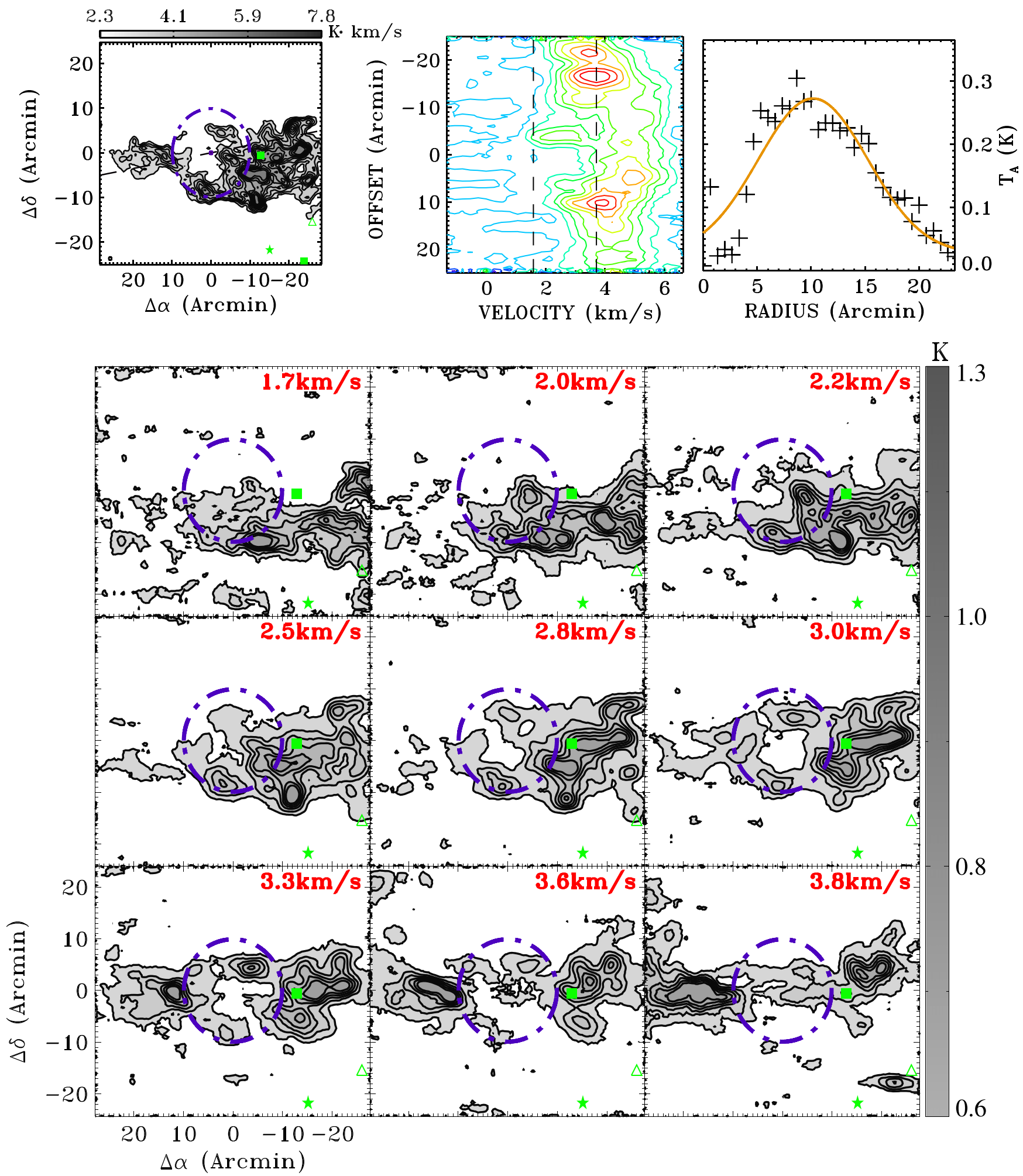

Figure 83. TMB_26. Same as Figure 58 except for the following. In the upper left panel, the integrated velocity interval is from 2.0 to $4.1 \mathrm{~km} \mathrm{~s}^{-1}$, the starting contour and the contour step are 1.5 and $0.8 \mathrm{~K} \mathrm{~km} \mathrm{~s}^{-1}$, respectively. In the upper middle panel, the position angle is $80^{\circ}$ and the contour levels are $0.1-0.6 \mathrm{~K}$ by $0.1 \mathrm{~K}$. 

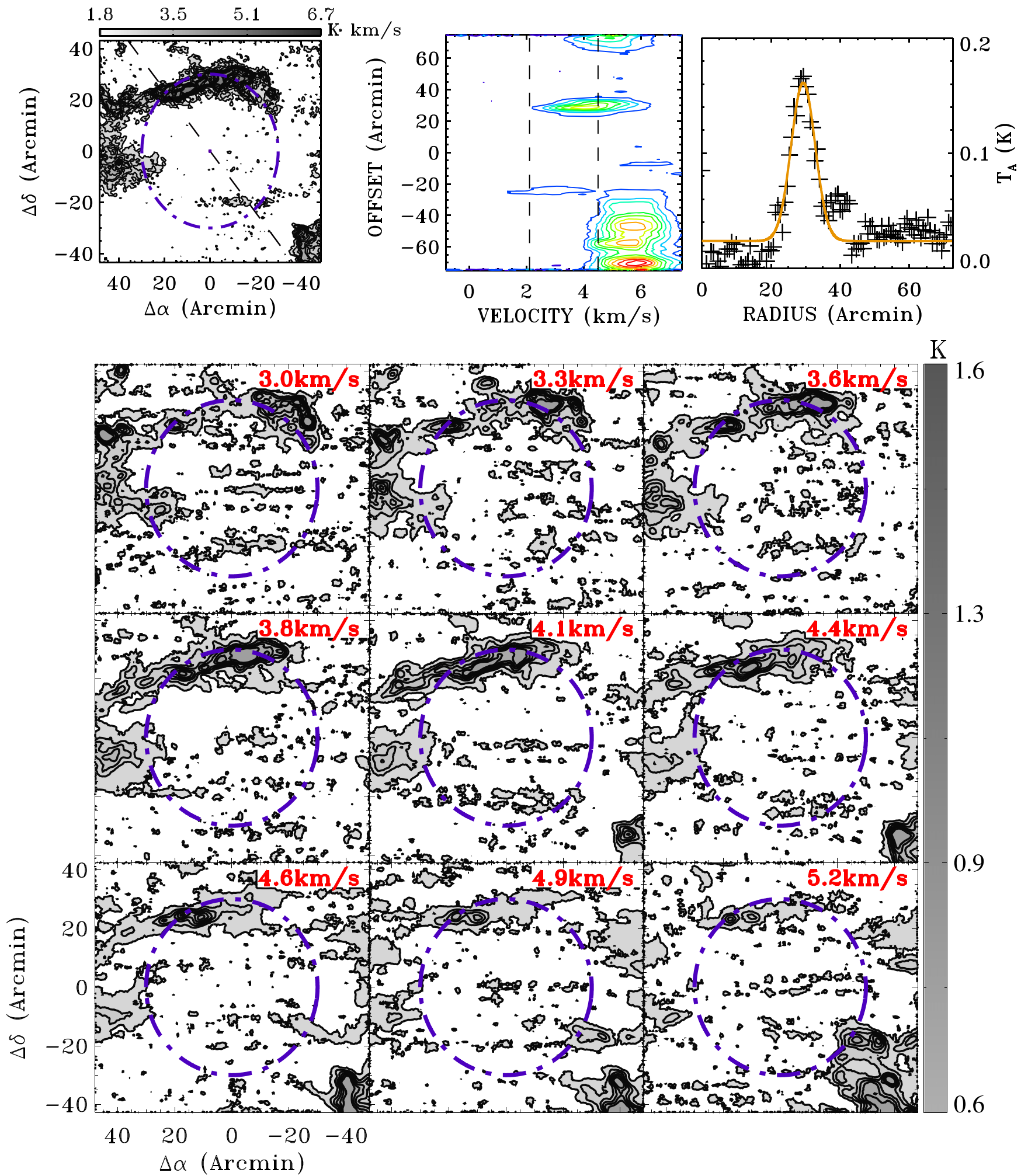

Figure 84. TMB_27. Same as Figure 58 except for the following. In the upper left panel, the integrated velocity interval is from 2.5 to $4.9 \mathrm{~km} \mathrm{~s}^{-1}$ and the starting contour is $1.8 \mathrm{~K} \mathrm{~km} \mathrm{~s}^{-1}$. In the upper middle panel, the position angle is $140^{\circ}$. 

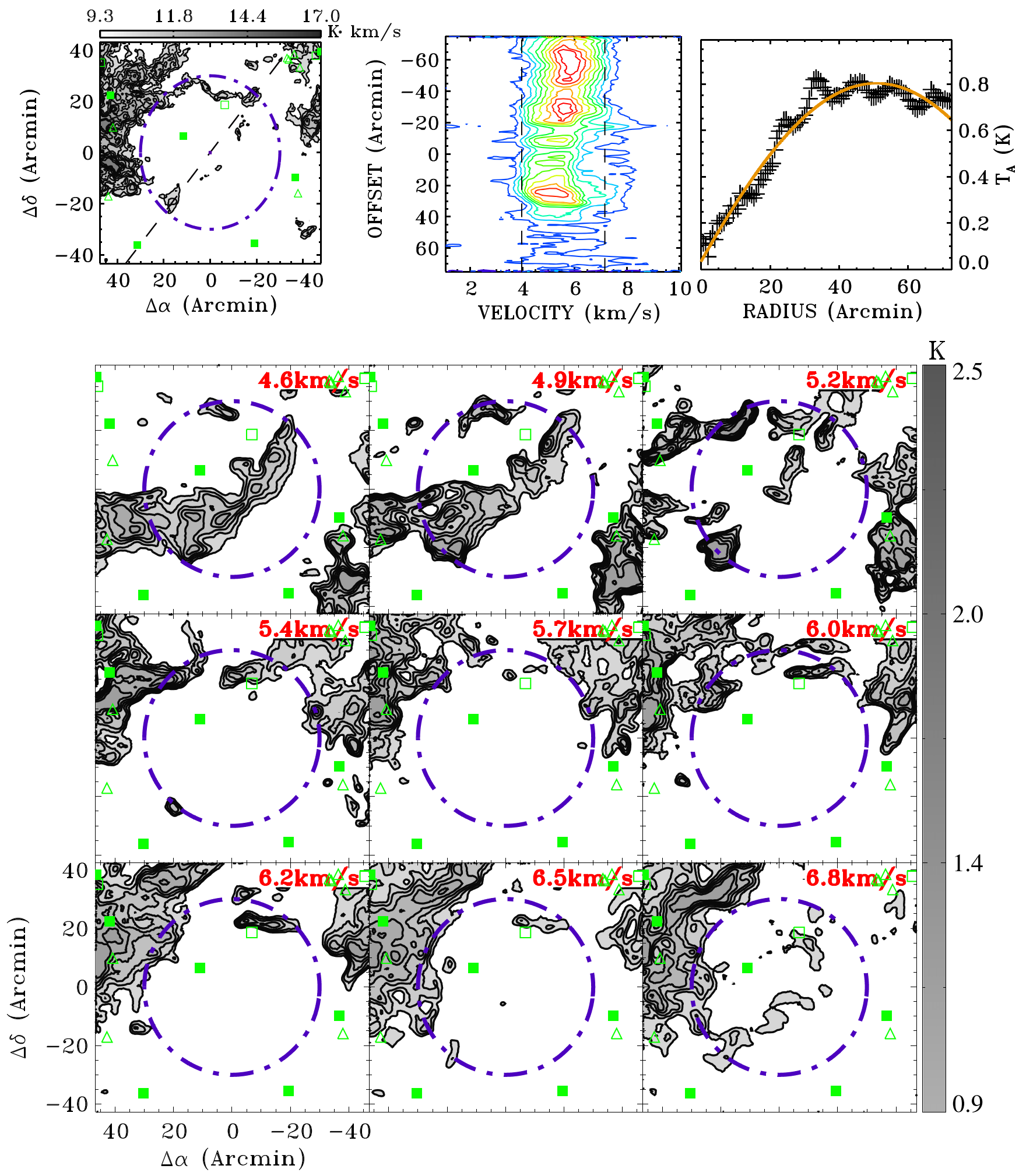

Figure 85. TMB_28. Same as Figure 58 except for the following. In the upper left panel, the integrated velocity interval is from 4.4 to $7.6 \mathrm{~km} \mathrm{~s}{ }^{-1}$, the starting contour and the contour step are 7.5 and $1.2 \mathrm{~K} \mathrm{~km} \mathrm{~s}^{-1}$, respectively. In the upper middle panel, the position angle is $40^{\circ}$. 

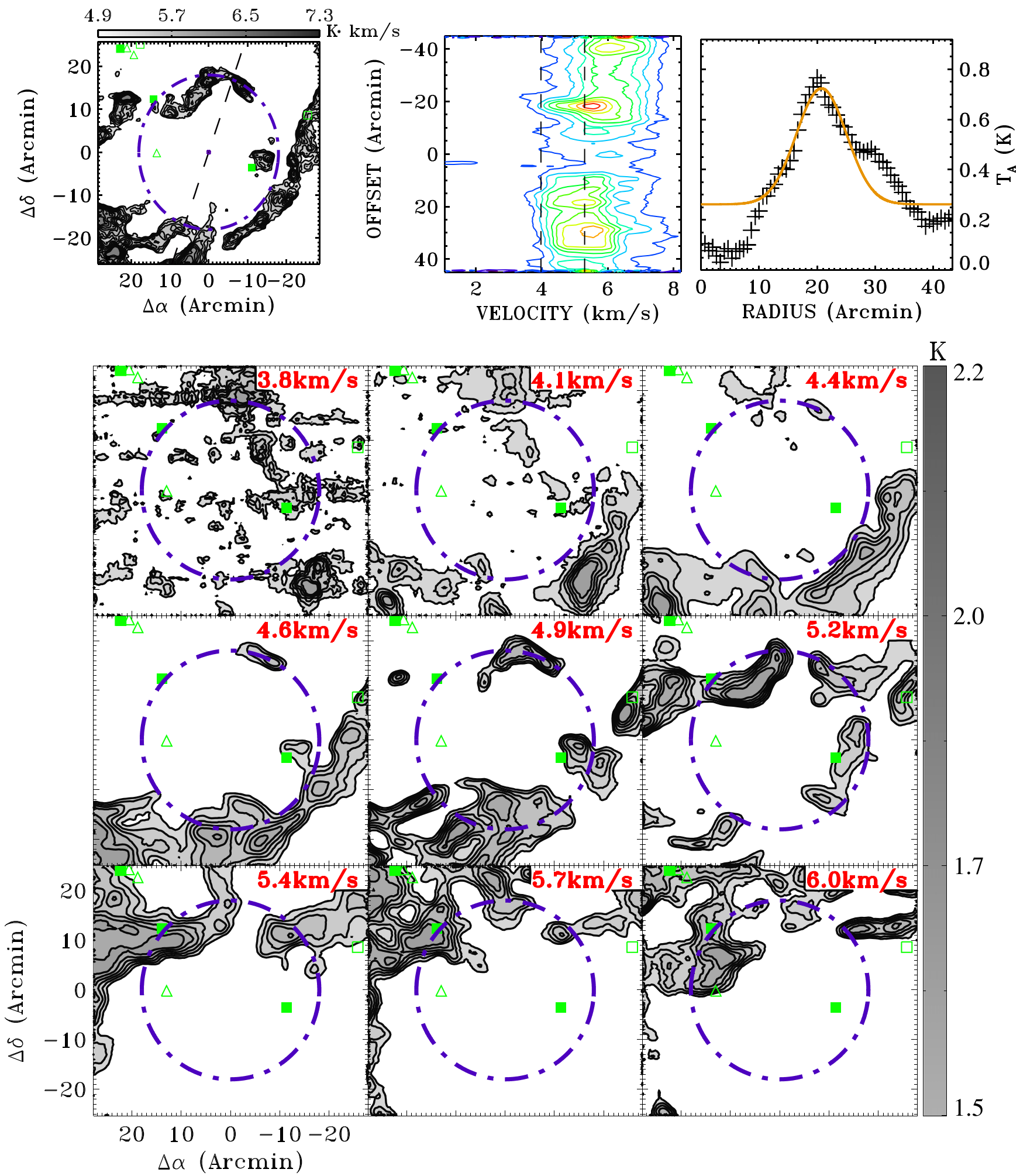

Figure 86. TMB_29. Same as Figure 58 except for the following. In the upper left panel, the integrated velocity interval is from 4.4 to $5.7 \mathrm{~km} \mathrm{~s}^{-1}$, the starting contour and the contour step are 4.0 and $0.4 \mathrm{~K} \mathrm{~km} \mathrm{~s}^{-1}$, respectively. In the upper middle panel, the position angle is $20^{\circ}$ and the contour levels are $0.1-1.3 \mathrm{~K}$ by $0.1 \mathrm{~K}$. 

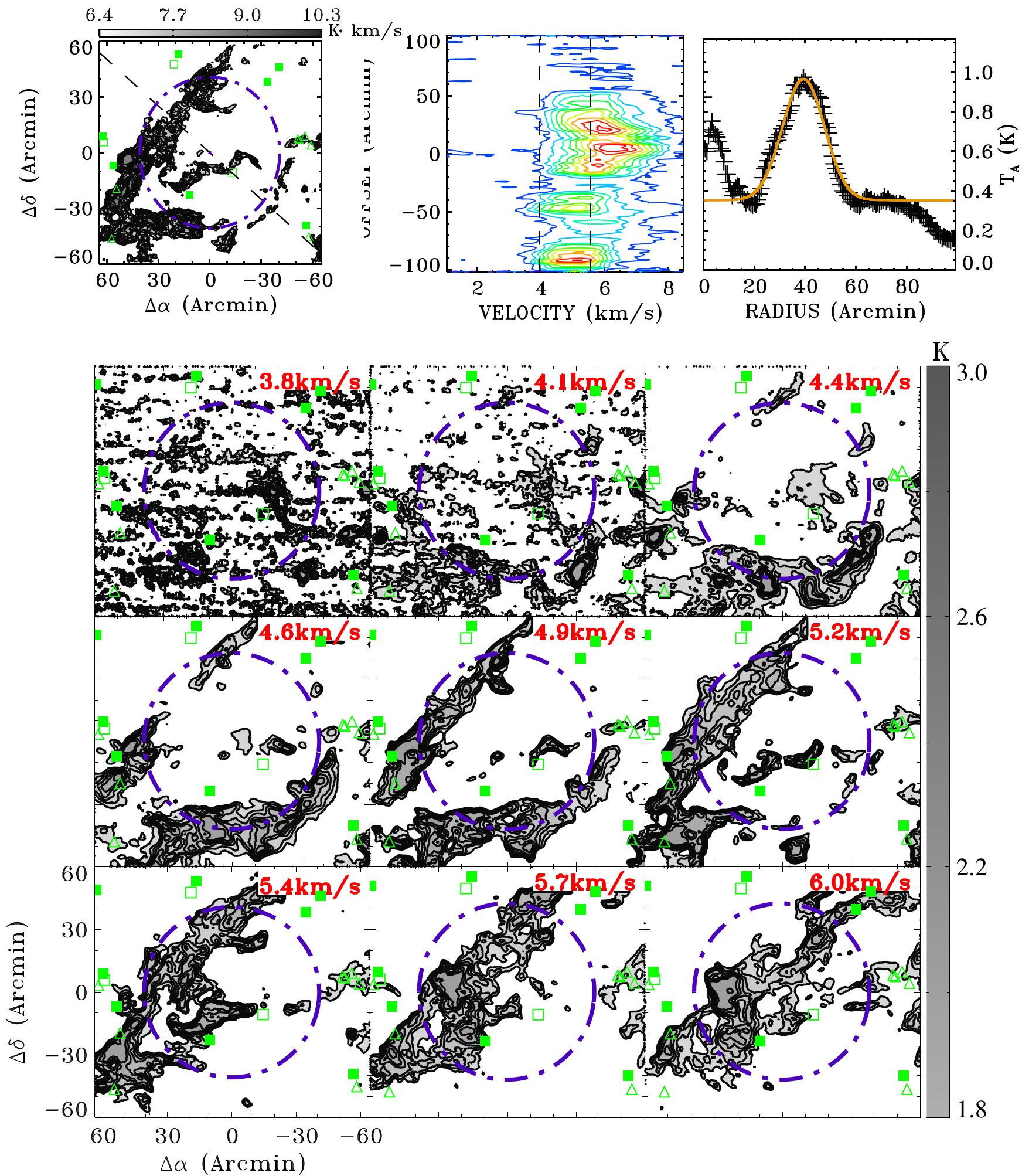

Figure 87. TMB_30. Same as Figure 58 except for the following. In the upper left panel, the integrated velocity interval is from 4.4 to $6.0 \mathrm{~km} \mathrm{~s}{ }^{-1}$ and the starting contour is $5.1 \mathrm{~K} \mathrm{~km} \mathrm{~s}^{-1}$. In the upper middle panel, the position angle is $130^{\circ}$ and the contour levels are $0.1-1.4 \mathrm{~K}$ by $0.1 \mathrm{~K}$. 

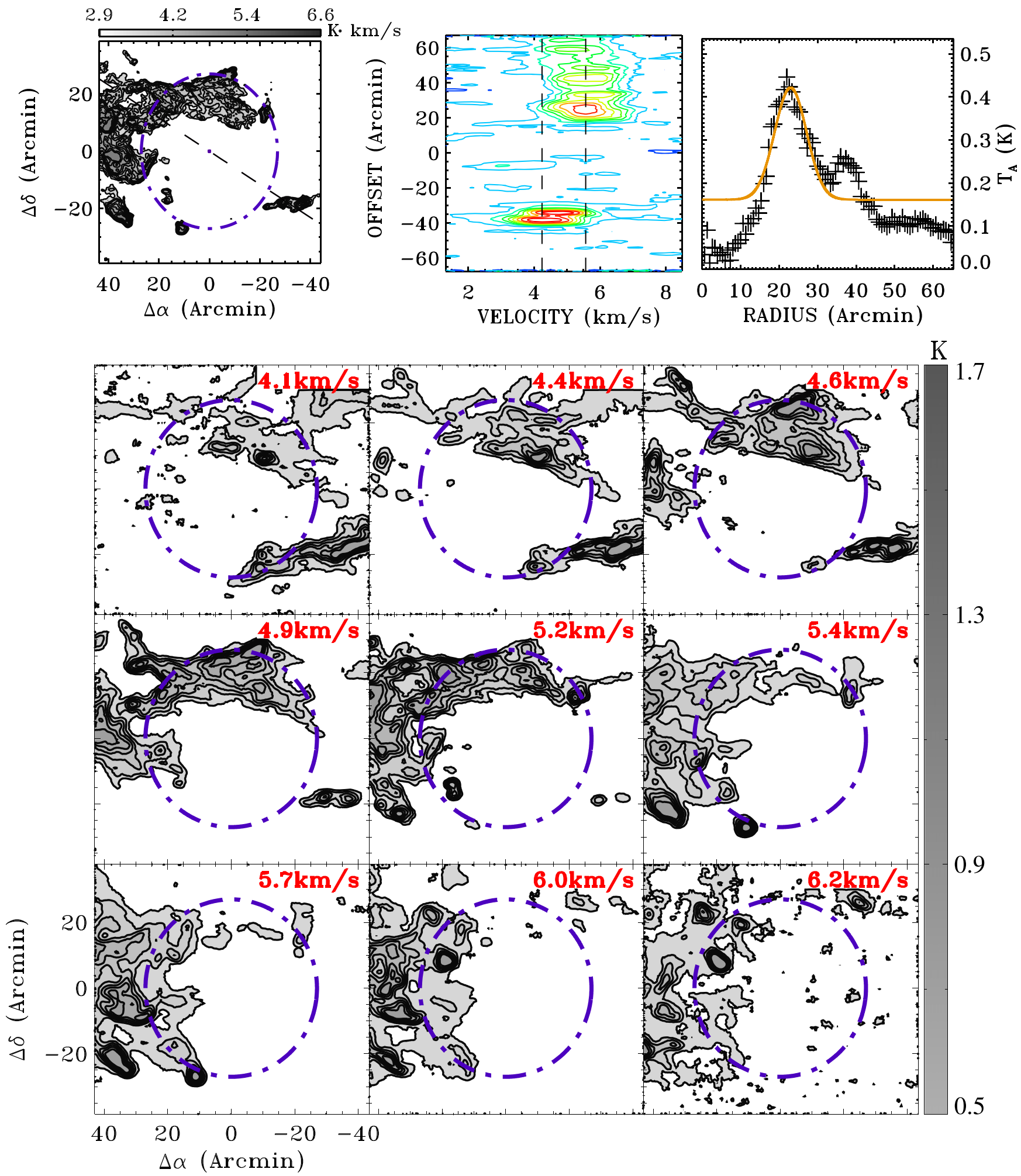

Figure 88. TMB 31. Same as Figure 58 except for the following. In the upper left panel, the integrated velocity interval is from 4.6 to $6.0 \mathrm{~km} \mathrm{~s} \mathrm{~s}^{-1}$ and the starting contour is $1.7 \mathrm{~K} \mathrm{~km} \mathrm{~s}^{-1}$. In the upper middle panel, the position angle is $120^{\circ}$ and the contour levels are $0.1-0.8 \mathrm{~K}$ by $0.1 \mathrm{~K}$. 

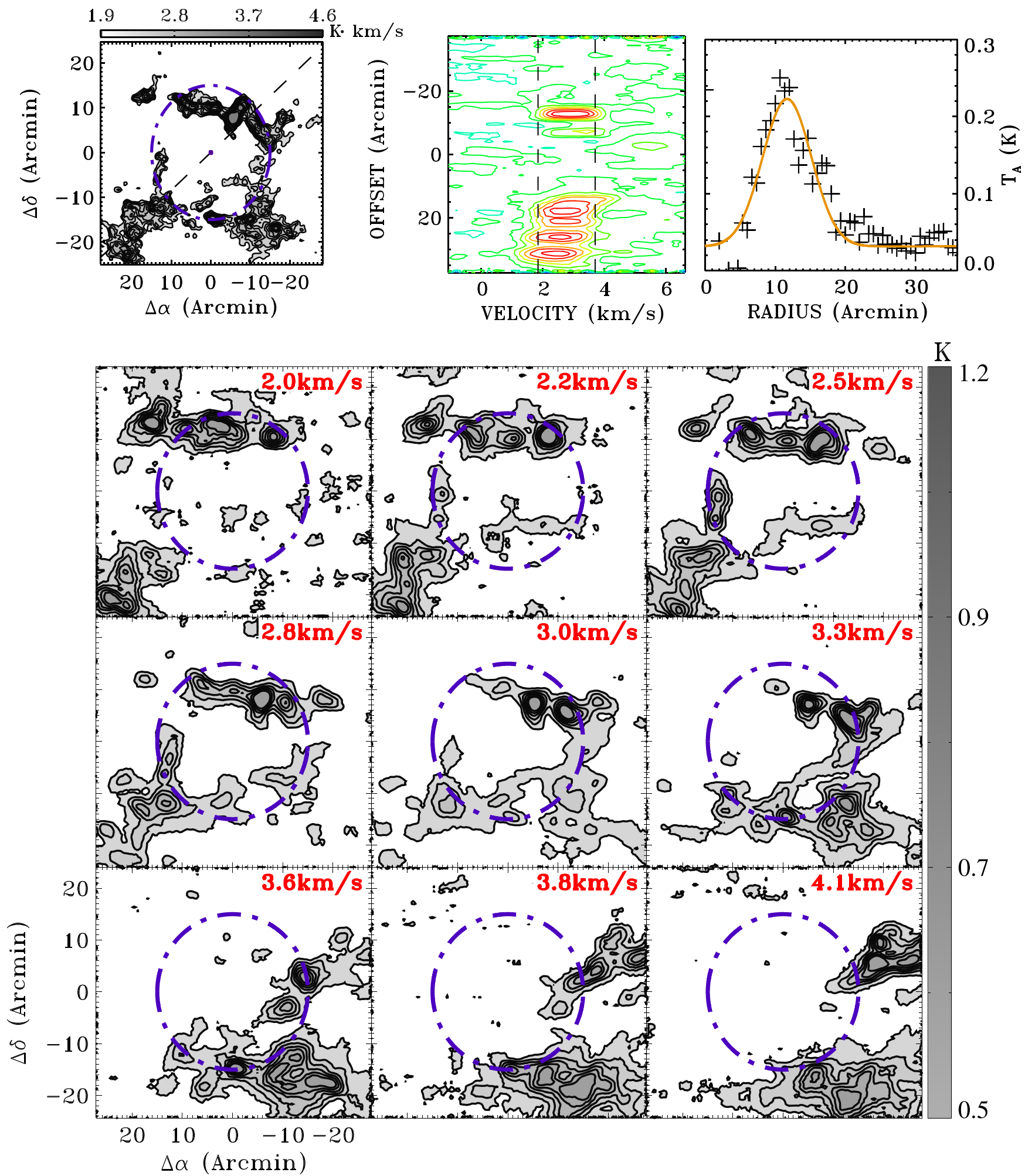

Figure 89. TMB 32. Same as Figure 58 except for the following. In the upper left panel, the integrated velocity interval is from 2.2 to $4.1 \mathrm{~km} \mathrm{~s}^{-1}$, the starting contour and the contour step are 1.4 and $0.4 \mathrm{~K} \mathrm{~km} \mathrm{~s}^{-1}$, respectively. In the upper middle panel, the position angle is $50^{\circ}$ and the contour levels are $0.1-0.5 \mathrm{~K}$ by $0.1 \mathrm{~K}$. 

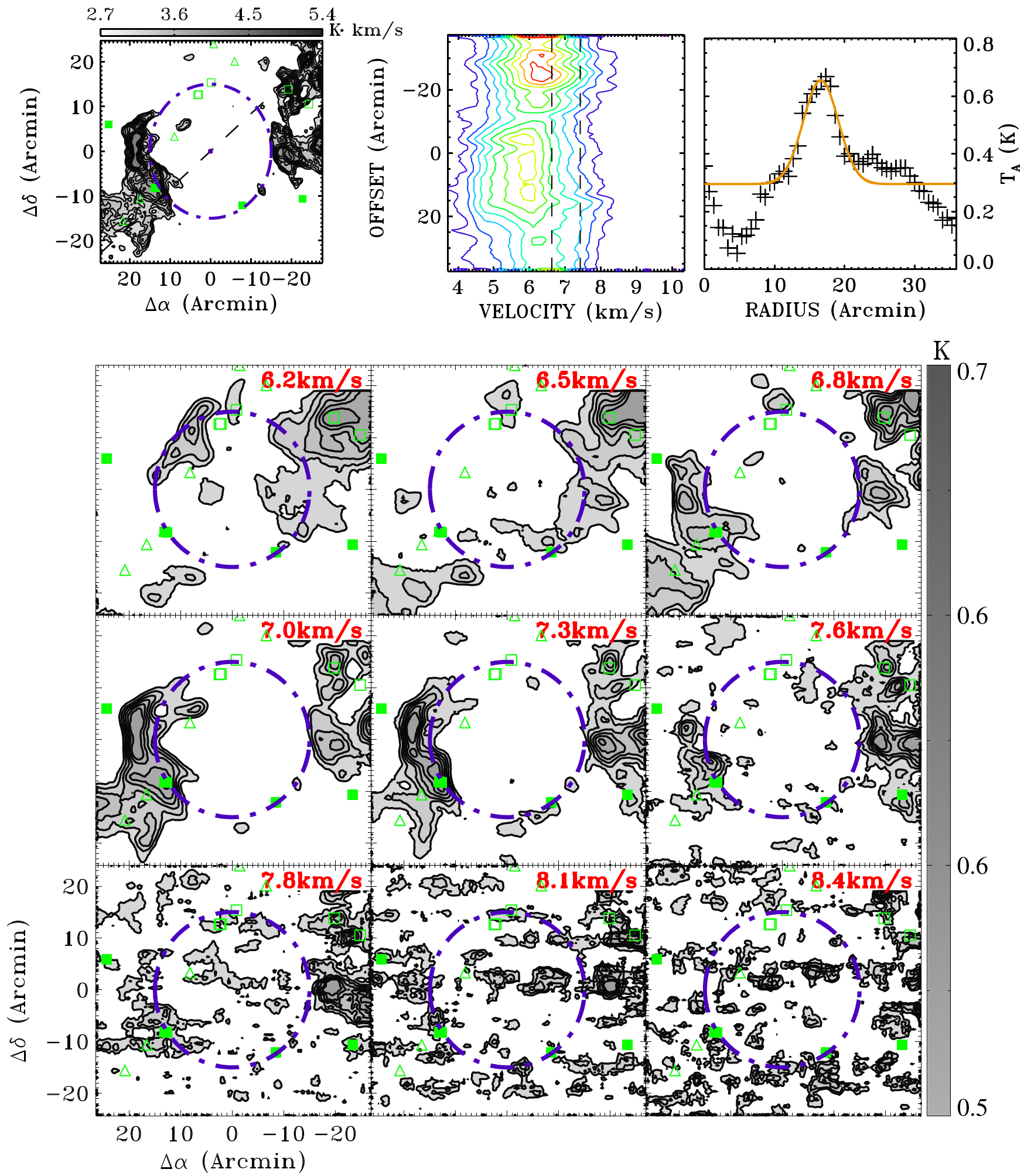

Figure 90. TMB_33. Same as Figure 58 except for the following. In the upper left panel, the integrated velocity interval is from 7.0 to $7.8 \mathrm{~km} \mathrm{~s}^{-1}$, the starting contour and the contour step are 2.0 and $0.4 \mathrm{~K} \mathrm{~km} \mathrm{~s}^{-1}$, respectively. In the upper middle panel, the position angle is $50^{\circ}$ and the contour levels are $0.1-2.2 \mathrm{~K}$ by $0.2 \mathrm{~K}$. 

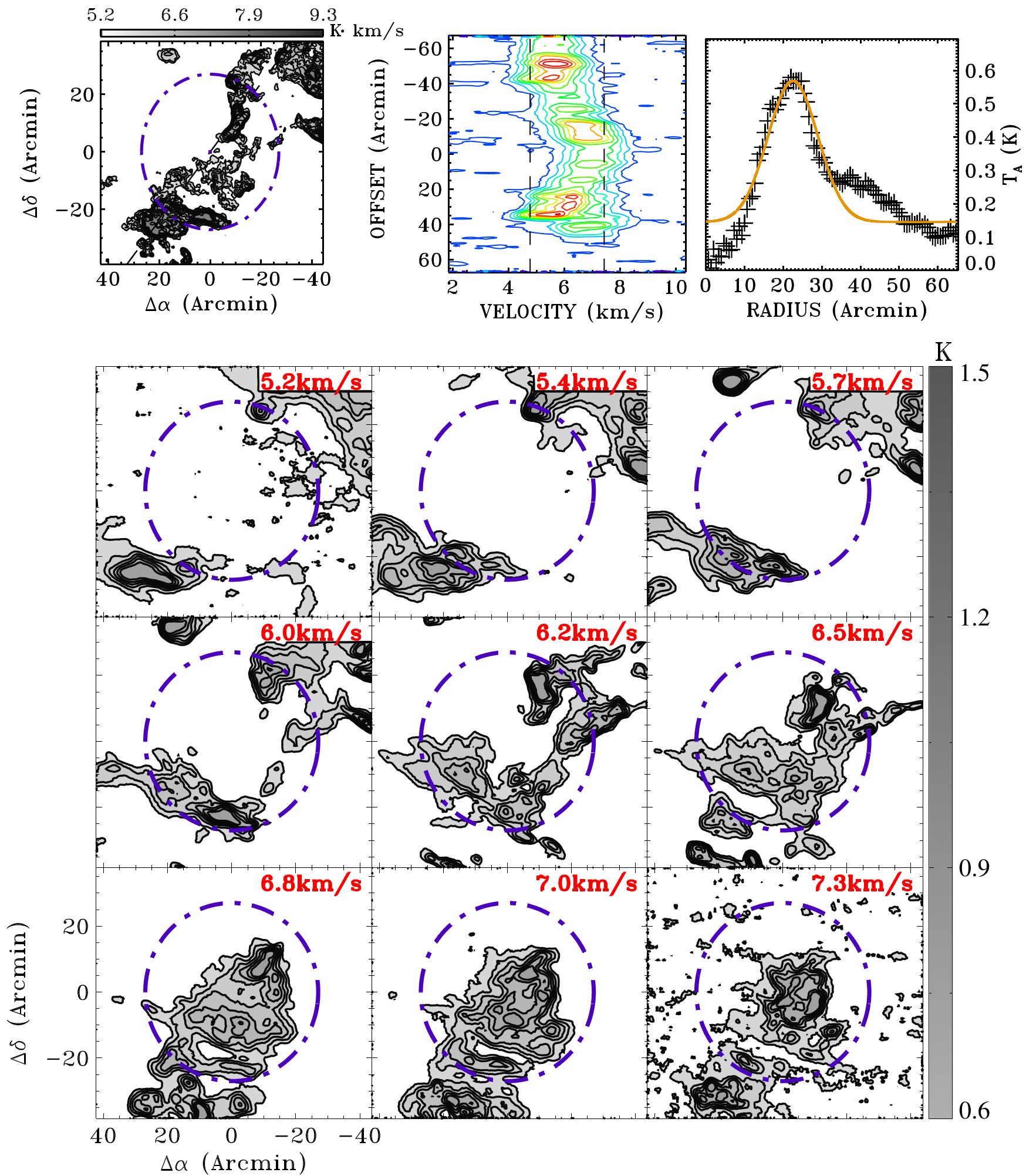

Figure 91. TMB 34. Same as Figure 58 except for the following. In the upper left panel, the integrated velocity interval is from 5.2 to $7.8 \mathrm{~km} \mathrm{~s}^{-1}$, the starting contour and the contour step are 3.8 and $0.7 \mathrm{~K} \mathrm{~km} \mathrm{~s}^{-1}$, respectively. In the upper middle panel, the position angle is $40^{\circ}$. 

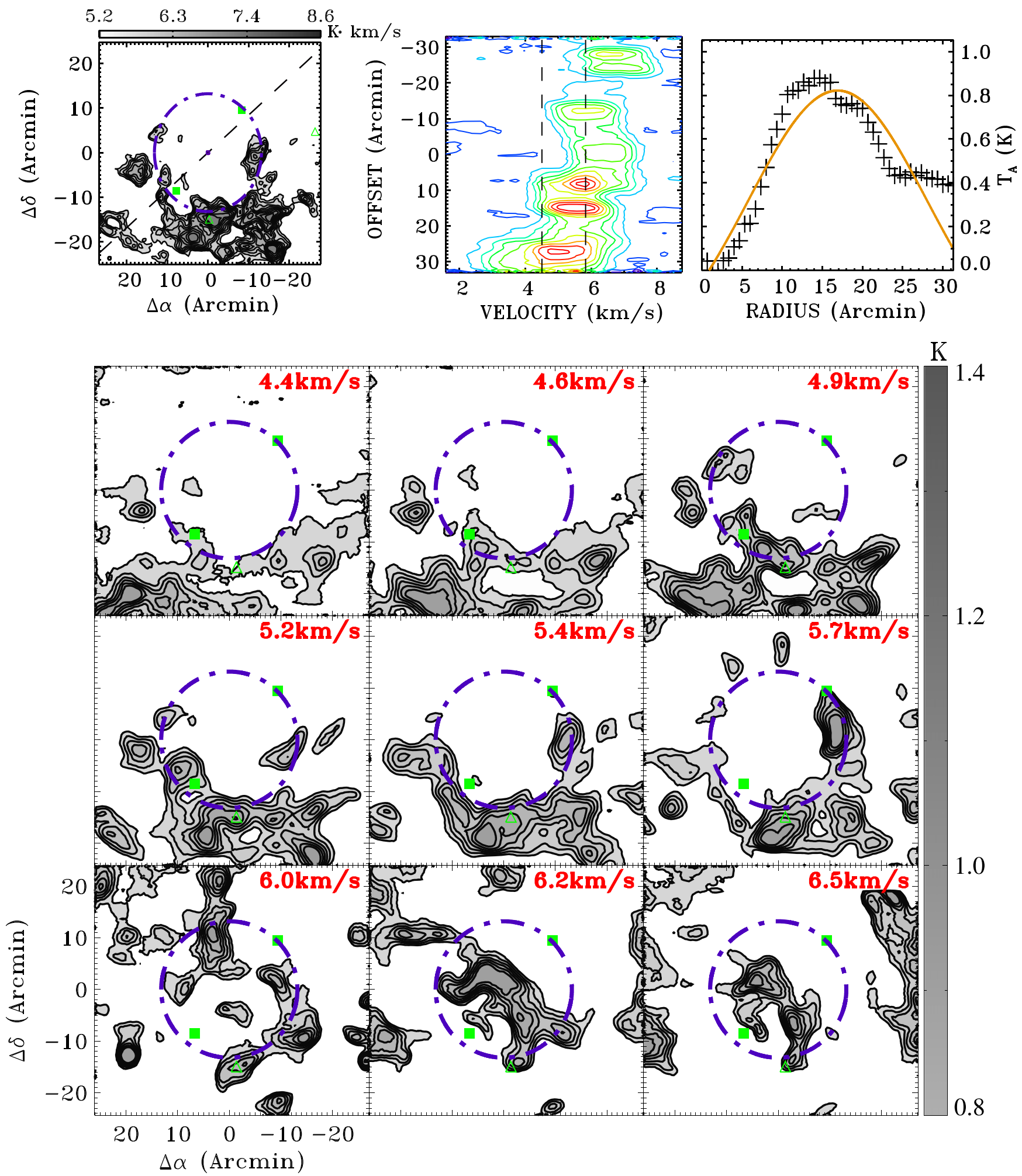

Figure 92. TMS 35. Same as Figure 58 except for the following. In the upper left panel, the integrated velocity interval is from 4.9 to $6.2 \mathrm{~km} \mathrm{~s}{ }^{-1}$ and the starting contour is $4.0 \mathrm{~K} \mathrm{~km} \mathrm{~s}^{-1}$. In the upper middle panel, the position angle is $50^{\circ}$ and the contour levels are $0.1-0.9 \mathrm{~K}$ by $0.1 \mathrm{~K}$. 

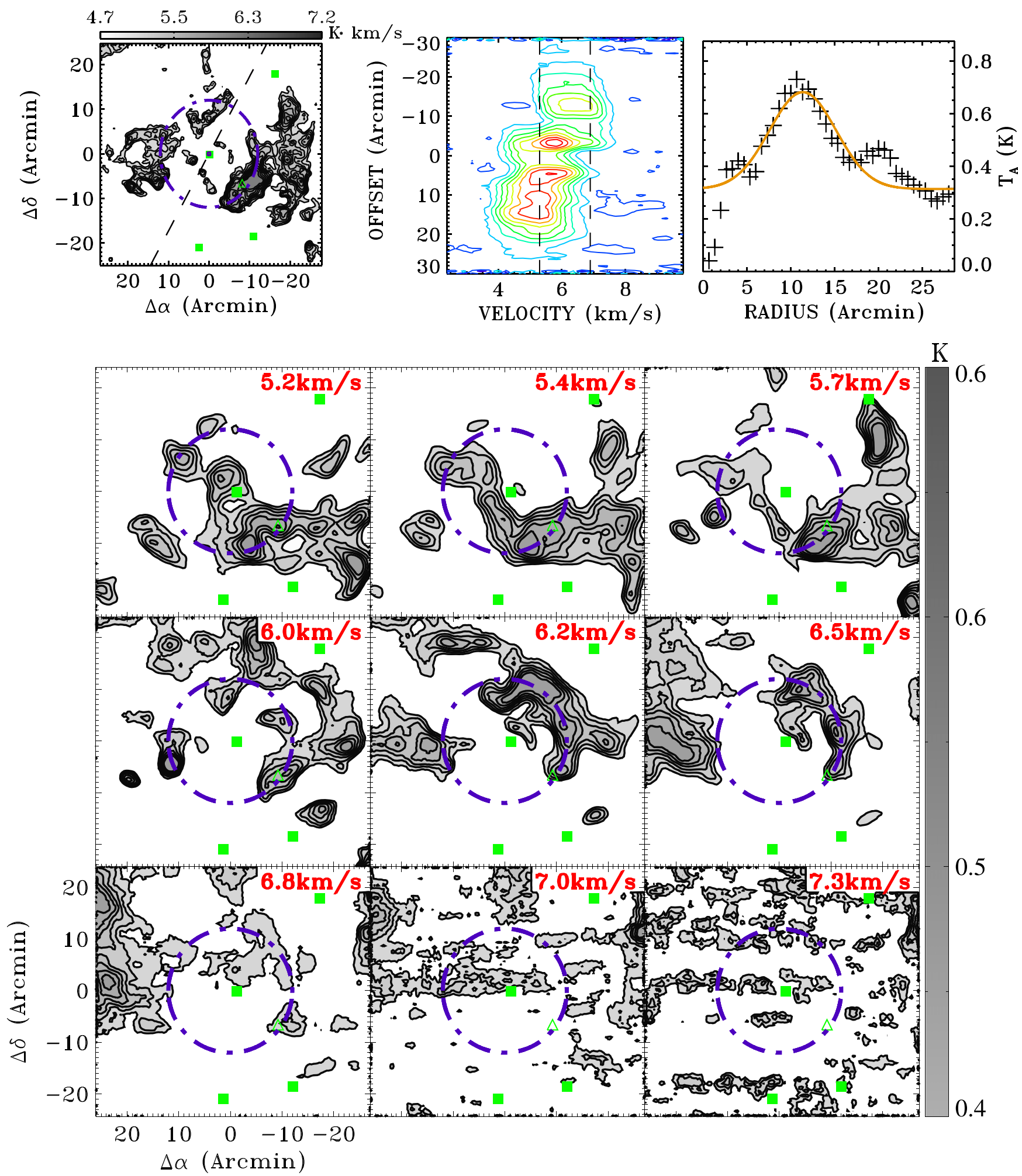

Figure 93. TMB_36. Same as Figure 58 except for the following. In the upper left panel, the integrated velocity interval is from 5.7 to $7.3 \mathrm{~km} \mathrm{~s}^{-1}$, the starting contour and the contour step are 4.0 and $0.4 \mathrm{~K} \mathrm{~km} \mathrm{~s}^{-1}$, respectively. In the upper middle panel, the position angle is $30^{\circ}$ and the contour levels are $0.1-0.8 \mathrm{~K}$ by $0.1 \mathrm{~K}$. 

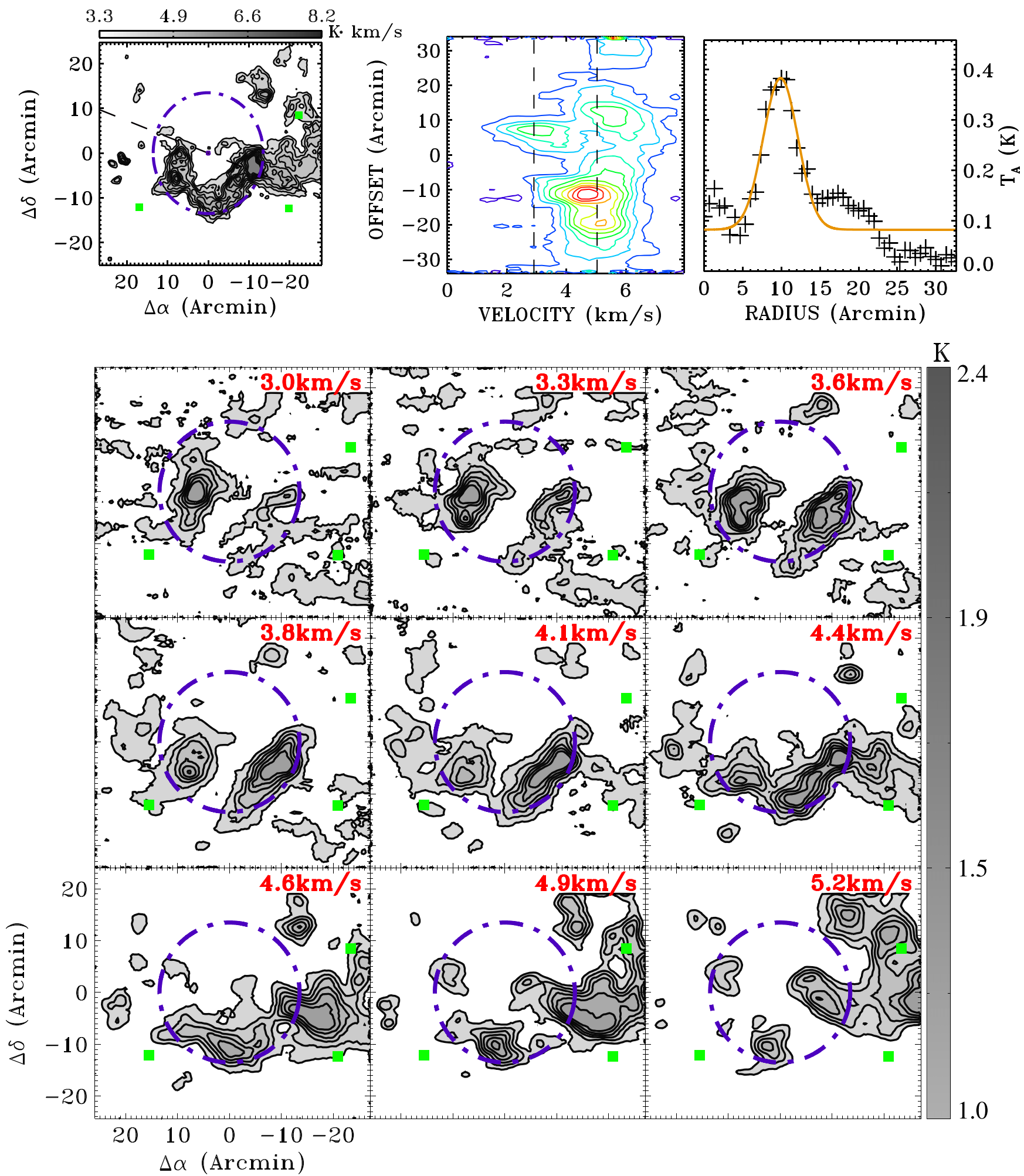

Figure 94. TMB 37. Same as Figure 58 except for the following. In the upper left panel, the integrated velocity interval is from 3.3 to $5.4 \mathrm{~km} \mathrm{~s}^{-1}$, the starting contour and the contour step are 2.2 and $0.7 \mathrm{~K} \mathrm{~km} \mathrm{~s}^{-1}$, respectively. In the upper middle panel, the contour levels are $0.1-1.1 \mathrm{~K} \mathrm{by} 0.1 \mathrm{~K}$. 

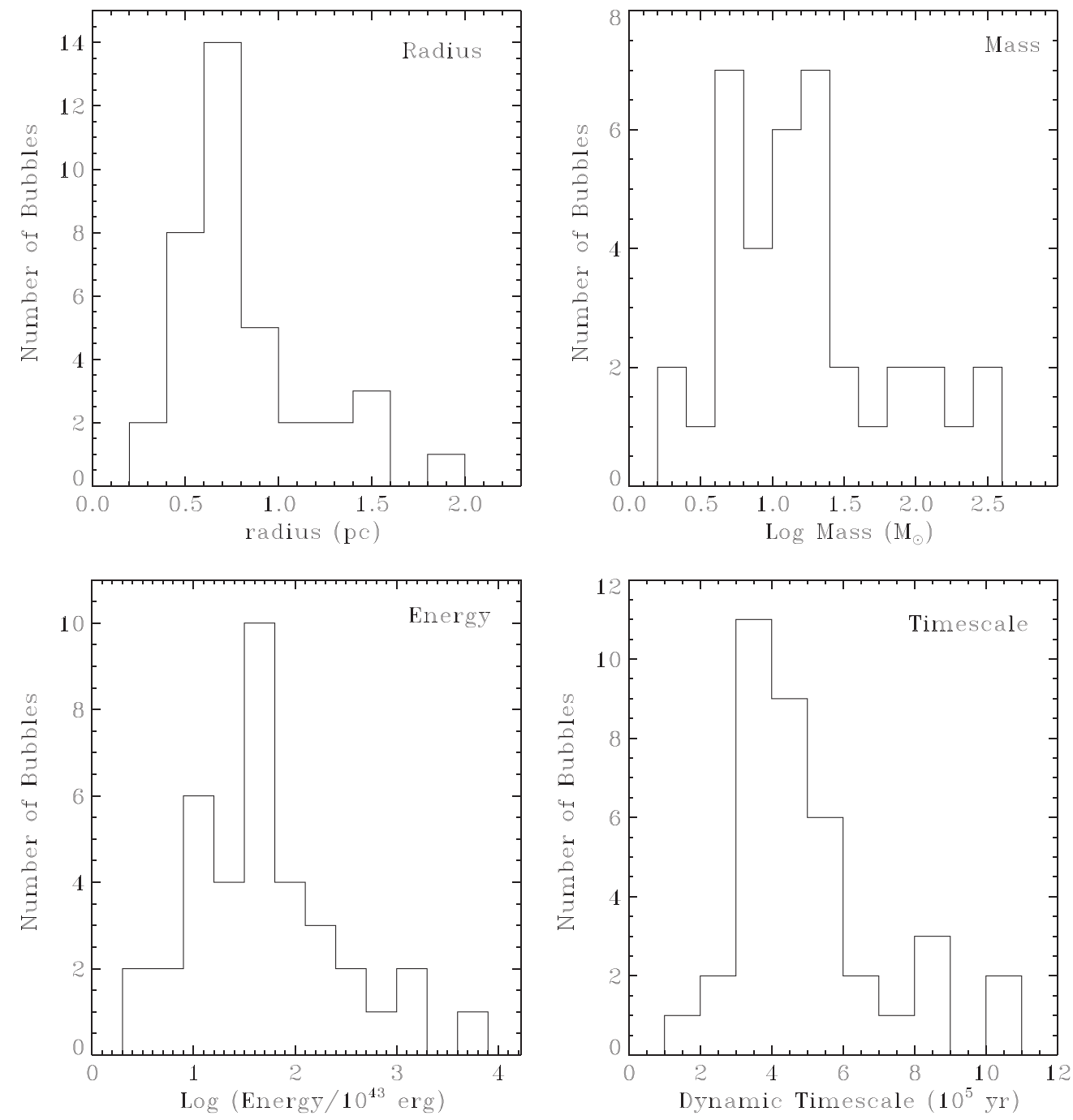

Figure 95. Histograms of the distributions of bubble parameters.

The Mach number of the turbulence can be calculated by (Mac Low 1999)

$$
M_{\mathrm{rms}}=\frac{\sigma_{3 \mathrm{~d}}}{c} .
$$

Using Equations (8), (15), and $c_{\delta}$ (16), we determine the Mach number, $M_{\mathrm{rms}}=5$, which is different from the assumption $\left(M_{\mathrm{rms}}=10\right)$ by Arce et al. (2010) and Narayanan et al. (2012).

The free-fall timescale of the cloud,

where

$$
t_{\mathrm{ff}}=\left(3 \pi / 32 G \rho_{\text {cloud }}\right)^{1 / 2},
$$

$$
\rho_{\text {cloud }}=\frac{3 M_{\text {cloud }}}{4 \pi R_{\text {cloud }}^{3}}
$$

is the average volume density of the cloud. Then, we obtain the free-fall timescale, $t_{\mathrm{ff}}=7 \times 10^{6}$ years.

Using the formulas from Equations (12) to (18), we determine the turbulent dissipation time to be $3.5 \times 10^{6}$ years. Then, we determine the turbulent dissipation rate to be $3.1 \times 10^{33} \mathrm{erg} \mathrm{s}^{-1}$, which is about 2.4 times larger than the luminosity of the outflows but $48 \%$ of the energy injection rate of bubbles. The turbulent dissipation rate we obtained is close to that $\left(3.8 \times 10^{33} \mathrm{erg} \mathrm{s}^{-1}\right)$ of Narayanan et al. (2012). In Table 8 , we list the parameters related to the dissipation rate we obtain from the above two methods and compare them with the results of Narayanan et al. (2012).

Both methods invoke numerical simulations to calibrate the numerical factors in addition to essentially dimensional arguments. The main difference of the two methods is the scale of the region where dissipation takes place. McKee \& Ostriker (2007) adopted the dimension of the entire cloud. Meanwhile, when we use the method provided by Mac Low (1999), the scale is the average size of the outflows and bubbles. None of the simulations so far implement the physics (excitation, radiative transfer, etc.) necessary for actually modeling dissipation. Thus, we should treat the calculations above with caution and take them as dimensional and order of magnitude estimates.

Comparing the energy injection rate of outflows and bubbles with the turbulent dissipation rate, we conclude that in the current episode of star formation in Taurus, both outflows and bubbles can sustain the currently observed turbulence in Taurus. 


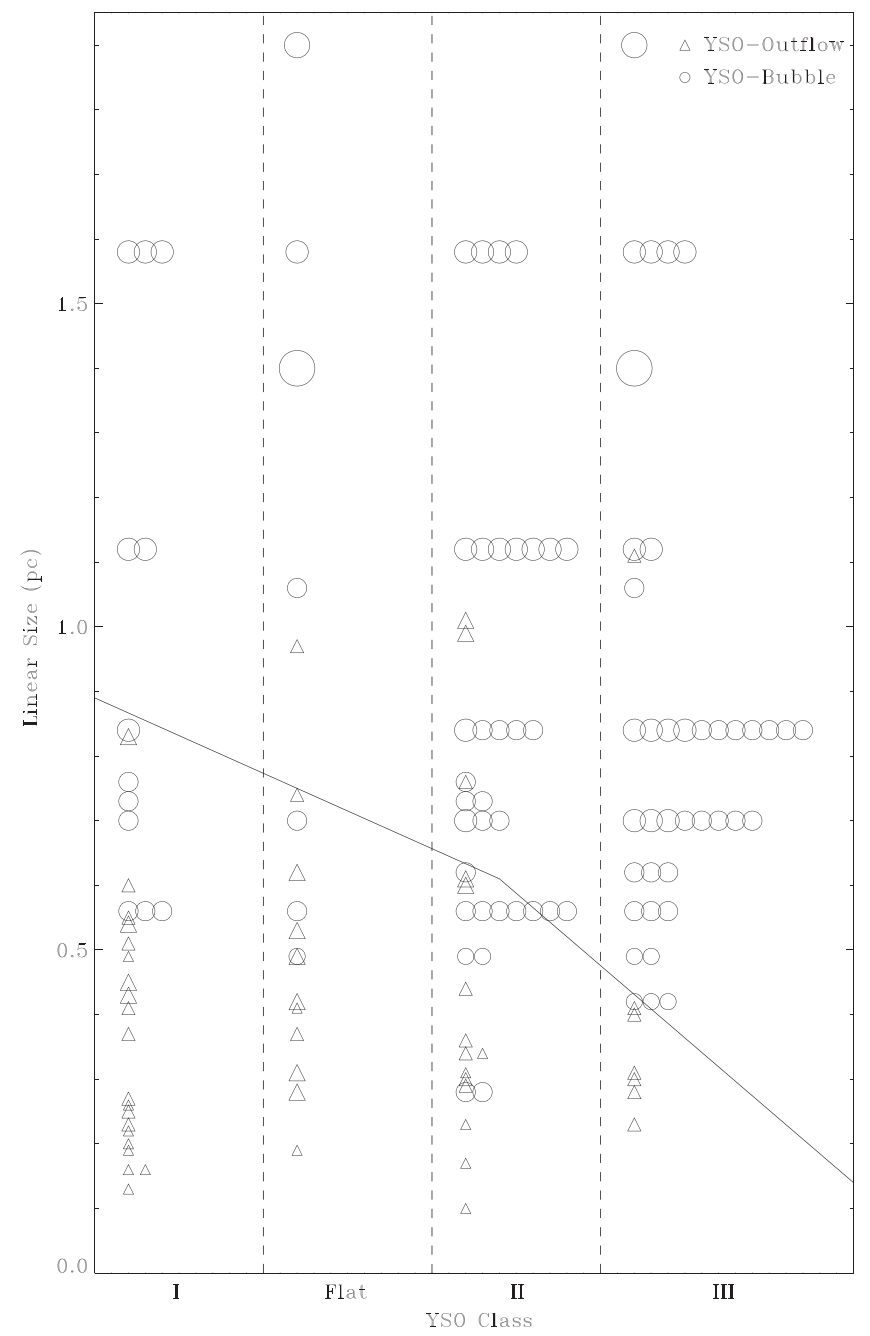

Figure 96. Distribution of Outflows and Bubbles. The open upward-pointing triangles represent the "outflow-driving YSOs" and the open circles represent the "bubble-driving YSOs." The black solid line roughly divides the outflows and bubbles. The size of the symbols is proportional to the energy of outflows and bubbles.

\subsection{Protostellar Winds Can Drive Bubbles to Sustain Turbulence in Taurus}

Protostellar winds will inject energy into the cloud and may help sustain turbulence (Nakamura \& Li 2007). The winds can clear the gas surrounding the young star and form a bubble structure (Arce et al. 2011). To assess whether the winds can drive bubbles in Taurus, we compared the wind energy injection rate into the cloud $\left(\dot{E}_{\mathrm{w}}\right)$ with the total energy injection rate from bubbles of the cloud. Following Arce et al. (2011), we estimated the wind energy injection rate using Equation (3.7) from McKee (1989):

$$
\dot{E}_{\mathrm{w}}=\frac{1}{2}\left(\dot{M}_{\mathrm{w}} v_{\mathrm{w}}\right) \sigma_{3 \mathrm{~d}},
$$

where $v_{\mathrm{w}}$ is the wind velocity, which is generally assumed to be close to the star escape velocity. For low- and intermediatemass stars, the escape velocity is about $(1-4) \times 10^{2} \mathrm{~km} \mathrm{~s}^{-1}$ (Lamers \& Cassinelli 1999). Similar to Arce et al. (2011), we assumed $v_{\mathrm{w}} \sim 200 \mathrm{~km} \mathrm{~s}^{-1}$. The total mass loss rate from the protostellar winds is given by $\dot{M}_{\mathrm{w}}$, which can be estimated by the sum of the wind mass loss rate for each bubble $\left(\dot{m}_{\mathrm{w}}\right)$. The wind mass loss rate required to produce the bubbles is roughly estimated by Equation (2) from Arce et al. (2011):

$$
\dot{m}_{\mathrm{w}}=\frac{P_{\text {bubble }}}{v_{\mathrm{w}} \tau_{\mathrm{w}}},
$$

where $P_{\text {bubble }}$ is the total momentum of the bubbles. The wind velocity, $v_{\mathrm{w}}$, is the same as that in Equation (19). $\tau_{\mathrm{w}}$ is the wind timescale, which is assumed to be $\sim 1$ Myr (Arce et al. 2011). From Equation (20), we obtained the wind mass loss rates of each bubble $\left(\dot{m}_{\mathrm{W}}\right)$, which are listed in Table 7 . Summing the $\dot{m}_{\mathrm{w}}$ of all bubbles, we find $\dot{M}_{\mathrm{w}}$ to be $1.89 \times 10^{-5} M_{\odot} \mathrm{yr}^{-1}$. Using Equation (19), we determined the wind energy injection rate $\left(\dot{E}_{\mathrm{w}}\right)$ to be $\sim 2 \times 10^{33} \mathrm{erg} \mathrm{s}^{-1}$, i.e., $31 \%$ of the total energy injection rate from bubbles in Taurus, which is comparable to the turbulent dissipation rate in Taurus. Therefore, the protostellar winds can drive bubbles to sustain turbulence in Taurus.

\subsection{Potential Sources of Turbulent Motions in Taurus}

The origin of turbulence in the molecular cloud has been intensely debated over the past three decades (e.g., Larson 1981; Heyer \& Brunt 2004). Hennebelle \& Falgarone 
Table 8

The Parameters Related to the Dissipation Rate

\begin{tabular}{lcccc}
\hline \hline Method & $\kappa$ & $M_{\text {rms }}$ & $\begin{array}{c}t_{\text {diss }} \\
\left(10^{6} \text { years }\right)\end{array}$ & $\begin{array}{c}L_{\text {turb }} \\
\left(10^{33} \text { erg s }^{-1}\right)\end{array}$ \\
\hline MO07 $^{\text {a }}$ & $\cdots$ & $\ldots$ & 16 & 0.7 \\
ML99 $^{\mathrm{b}}$ & 0.64 & 5 & 3.5 & 3.1 \\
Na12 $^{\mathrm{c}}$ & 1 & 10 & 2.7 & 3.8 \\
\hline
\end{tabular}

Notes.

a The result we obtain using the method given by McKee \& Ostriker (2007).

b The result we obtain using the method given by Mac Low (1999).

c The result of Narayanan et al. (2012) using the method given by Mac Low (1999).

(2012) suggest that for a large fraction of clouds, the turbulent driving is external. Numerical simulations show that the external sources of turbulence are likely to be large-scale $\mathrm{H}$ I streams (Ballesteros-Paredes et al. 1999), shocks (McKee \& Ostriker 2007), Alfvén waves (Nakamura \& Li 2007; Wang et al. 2010), supernova explosions, and galactic differential rotation (Klessen \& Hennebelle 2010; Hennebelle \& Falgarone 2012). It is unclear which of these is the source of the turbulence in Taurus.

\section{CONCLUSIONS}

We have studied the dynamic structures including outflows and bubbles within the Taurus molecular cloud using the $100 \mathrm{deg}^{2}$ FCRAO large-scale ${ }^{12} \mathrm{CO}(1-0)$ and ${ }^{13} \mathrm{CO}(1-0)$ maps and the Spitzer protostellar catalog. The high sensitivity and large spatial dynamic range of the maps provide us with an excellent opportunity to undertake an unbiased search for outflows and bubbles in this region. We also analyzed the energy injection of these dynamic structures into the entire cloud. Our conclusions regarding the dynamic structures in Taurus and their properties are as follow.

1. We identified 55 outflows around the Spitzer YSOs in the main $44 \mathrm{deg}^{2}$ area of Taurus. In total, 31 of the detected outflows were previously unknown, increasing the number of outflows by a factor of 1.3 .

2. We classified the outflows into five categories according to the morphology of contour maps and $\mathrm{P}-\mathrm{V}$ diagrams. The classifications indicate the confidence level of the outflows. $76.3 \%$ of the outflows are in the "most probable" category in our study.

3. Most of the outflows are driven by Class I, Flat, and Class II YSOs, while few outflows were found around Class III YSOs, which indicates that the outflow activity likely occurred in the earlier stage rather than the late phase of star formation.

4. More bipolar and monopolar redshifted outflows were identified while few monopolar blueshifted ones were detected in our study.

5. We detected 37 bubbles in the $100 \mathrm{deg}^{2}$ region of Taurus. All of the bubbles were previously unknown. The bubbles were identified by the integrated intensity maps, $\mathrm{P}-\mathrm{V}$ diagrams, Gaussian fitting profiles, and channel maps.

6. The gravitational binding energy of the Taurus molecular cloud is $\sim 1.5 \times 10^{48} \mathrm{erg}$. The total kinetic energy of outflows and bubbles in Taurus are $\sim 3.9 \times 10^{45} \mathrm{erg}$ and $\sim 9.2 \times 10^{46} \mathrm{erg}$, respectively. Neither outflows nor bubbles can balance the overall gravitational binding energy of Taurus.

7. The turbulent energy of the Taurus molecular cloud is $\sim 3.2 \times 10^{47} \mathrm{erg}$. The energy of all of the detected outflows and bubbles cannot have generated the observed turbulence in Taurus.

8. The rate of turbulent dissipation in Taurus ranges from $\sim 6.6 \times 10^{32}$ to $\sim 3.1 \times 10^{33} \mathrm{erg} \mathrm{s}^{-1}$. The energy injection rates of outflows and bubbles are $\sim 1.3 \times 10^{33}$ $\mathrm{erg} \mathrm{s}^{-1}$ and $\sim 6.4 \times 10^{33} \mathrm{erg} \mathrm{s}^{-1}$, respectively. Both outflows and bubbles can sustain the turbulence in Taurus at the current epoch.

9. The stellar winds can drive bubbles to sustain turbulence in the Taurus molecular cloud.

We are grateful to Dr. Y. L. Yue, Dr. Z. Y. Zhang, Dr. T. Liu, Dr. X. Y. Gao, and Dr. Z. Y. Ren for their kind and valuable advice and support. We thank the anonymous referee for a careful inspection of the manuscript and constructive comments, particularly the important suggestion to examine the turbulent dissipation issue in order to improve the quality of this study. We also thank Prof. W. Butler Burton for help in the review process. This work is partly supported by the China Ministry of Science and Technology under State Key Development Program for Basic Research (2012CB821802), and the National Natural Science Foundation of China (11373038, 11373045), the Hundred Talents Program of the Chinese Academy of Sciences, and the Young Researcher Grant of National Astronomical Observatories, Chinese Academy of Sciences.

\section{APPENDIX \\ DERIVATIONS OF OUTFLOW PARAMETERS}

To calculate molecular outflow parameters, we need first to obtain the column density. A simple solution of the equation of radiative transfer is

$$
T_{\mathrm{s}}=\left[T_{\mathrm{ex}}-T_{\mathrm{bg}}\right]\left(1-e^{-\tau_{\nu}}\right)
$$

where $T_{\mathrm{s}}$ is the source temperature, $T_{\mathrm{ex}}$ is the excitation temperature, $T_{\mathrm{bg}}$ is the background temperature, and $\nu$ is the frequency of the transition. The modified Planck function $J$ is defined as

$$
J(T)=\frac{h \nu / k}{e^{\frac{h \nu}{k T}}-1},
$$

where $k$ is Boltzmann's constant and $h$ is Planck's constant. The definition of optical depth in terms of upper level column density is expressed as (Wilson et al. 2013)

$$
\int \tau_{\nu} d \nu=\frac{A_{\mathrm{ul}} c^{2} N_{\mathrm{u}}}{8 \pi \nu^{2}}\left(e^{h \nu / k T_{\mathrm{ex}}}-1\right) .
$$

Assuming $\tau \ll 1, h \nu / k \ll T_{\text {ex }}$, and $T_{\text {bg }} \ll T_{\text {ex }}$, we obtain the column density of the rotational upper level of the transition in the outflow by combining Equation (21) with Equation (23).

$$
N_{\mathrm{u}}\left({ }^{12} \mathrm{CO}\right)=\frac{8 \pi k \nu^{2}}{h c^{3} A_{\mathrm{ul}}} \int T_{\mathrm{s}} d \nu,
$$

where $T_{\mathrm{s}}$ is the observed source antenna temperature with proper correction for antenna efficiency, $c$ is the speed of light, 
and $A_{\mathrm{ul}}$ is the spontaneous transition rate from the upper level $(J+1)$ to the lower level $(J)$, which can be expressed as

$$
A_{\mathrm{ul}}=\frac{64 \pi^{4} \nu^{3} \mu_{\mathrm{d}}^{2}}{3 h c^{3}} \frac{J+1}{2 J+3},
$$

where $\mu_{\mathrm{d}}$ is the permanent electric dipole moment of a molecule and $J=0$.

The total column density of the outflow is

$$
N_{\text {tot }}\left({ }^{12} \mathrm{CO}\right)=\frac{N_{\mathrm{u}}\left({ }^{12} \mathrm{CO}\right)}{f_{\mathrm{u}}},
$$

where $f_{\mathrm{u}}$ is the fraction of the ${ }^{12} \mathrm{CO}$ in the upper level of the transition. Under local thermal equilibrium (LTE) conditions, $f_{\mathrm{u}}$ is given by

$$
f_{\mathrm{u}}=\frac{g_{\mathrm{u}} \exp \left(-h \nu / k T_{\mathrm{ex}}\right)}{Q\left(T_{\mathrm{ex}}\right)},
$$

where the statistical weight of the upper level $g_{\mathrm{u}}=2$ $(J+1)+1$, the LTE partition function (for $k T_{\text {ex }} \gg h B$ ) $Q\left(T_{\mathrm{ex}}\right)=k T_{\mathrm{ex}} / h B$, and the rotational constant $B=\nu /[2$ $(J+1)]$ for the $J+1 \rightarrow J$ transition (Tennyson 2005). Then, we can derive the total column density of outflow from Equations (24)-(27),

$$
N_{\text {tot }}\left({ }^{12} \mathrm{CO}\right)=\frac{3 k^{2} T_{\mathrm{ex}}}{4 \pi^{3} \mu_{\mathrm{d}}^{2} h \nu^{2} \exp \left(-h \nu / k T_{\mathrm{ex}}\right)} \int T_{\mathrm{s}} d \nu .
$$

If there is a high-velocity wing in ${ }^{12} \mathrm{CO}$ but not in the ${ }^{13} \mathrm{CO}$ profile, we assume that ${ }^{12} \mathrm{CO}$ is optically thin. Then, we can calculate the column density of the outflow from Equation (28). If there is a high-velocity wing in both the ${ }^{12} \mathrm{CO}$ and ${ }^{13} \mathrm{CO}$ profiles, then we can correct the optical depth of ${ }^{12} \mathrm{CO}$ using the following equation:

$$
\frac{T_{\mathrm{a}}^{*}\left({ }^{12} \mathrm{CO}\right)}{T_{\mathrm{a}}^{*}\left({ }^{13} \mathrm{CO}\right)}=\frac{1-e^{-\tau\left({ }^{12} \mathrm{CO}\right)}}{1-e^{-\tau\left({ }^{(13} \mathrm{CO}\right)}} .
$$

Here $T_{\mathrm{a}}^{*}\left({ }^{12} \mathrm{CO}\right)$ and $T_{\mathrm{a}}^{*}\left({ }^{13} \mathrm{CO}\right)$ are the antenna temperatures of ${ }^{12} \mathrm{CO}$ and ${ }^{13} \mathrm{CO}$ (with proper correction for antenna efficiency), respectively. $\tau\left({ }^{12} \mathrm{CO}\right)$ and $\tau\left({ }^{13} \mathrm{CO}\right)$ are the optical depths of ${ }^{12} \mathrm{CO}$ and ${ }^{13} \mathrm{CO}$, respectively. We assume the abundance ratio of ${ }^{12} \mathrm{CO}$ to ${ }^{13} \mathrm{CO}$ is 65 (Langer \& Penzias 1993). The correction factor for opacity is defined as

$$
f_{\tau}=\frac{\tau\left({ }^{12} \mathrm{CO}\right)}{1-e^{-\tau\left({ }^{12} \mathrm{CO}\right)}} .
$$

Then, we determine the corrected total column density of the outflow as

$$
N_{\text {ctot }}\left({ }^{12} \mathrm{CO}\right)=f_{\tau} N_{\text {tot }}\left({ }^{12} \mathrm{CO}\right) .
$$

After obtaining the column density, we can calculate other parameters of the outflow. The mass of the outflow can be calculated from

$$
M_{\text {gas }}=N_{\text {tot }}\left({ }^{12} \mathrm{CO}\right)\left[\mathrm{H}_{2} / \mathrm{CO}\right] \mu_{\mathrm{g}} m(\mathrm{H}) S,
$$

where $\mu_{\mathrm{g}}=2.72$ is the mean molecular weight (Brunt 2010), $m(\mathrm{H})=1.67 \times 10^{-24} \mathrm{~g}$ is the mass of a hydrogen atom,
$\left[\mathrm{H}_{2}\right] /[\mathrm{CO}]$ is assumed to be $10^{4}$, and $S$ is the area of the outflow.

The momentum $(P)$ and energy $(E)$ of the outflow can be calculated from

$$
\begin{gathered}
P=M_{\mathrm{gas}}|\bar{v}|, \\
E=\frac{1}{2} M_{\mathrm{gas}} \bar{v}^{2},
\end{gathered}
$$

where $\bar{v}$ is the average velocity of the outflow relative to the cloud systemic velocity and $M_{\text {gas }}$ is obtained from Equation (32).

The dynamical timescale $t_{\mathrm{dyn}}$ can be estimated from

$$
t_{\mathrm{dyn}}=\frac{L}{|\bar{v}|},
$$

where $L$ is the typical linear scale of the outflow lobe. The outflow luminosity, $L_{\text {flow }}$, can be estimated by dividing the kinetic energy by the dynamical timescale. It can be expressed as

$$
L_{\text {flow }}=\frac{E}{t_{\text {dyn }}} \text {. }
$$

\section{REFERENCES}

Arce, H. G., Borkin, M. A., Goodman, A. A., Pineda, J. E., \& Beaumont, C. N. 2011, ApJ, 742, 105

Arce, H. G., Borkin, M. A., Goodman, A. A., Pineda, J. E., \& Halle, M. W. 2010, ApJ, 715, 1170

Arce, H. G., \& Goodman, A. A. 2001, ApJ, 554, 132

Bachiller, R. 1996, ARA\&A, 34, 111

Ballesteros-Paredes, J., Hartmann, L., \& Vázquez-Semadeni, E. 1999, ApJ, 527,285

Beaumont, C. N., \& Williams, J. P. 2010, ApJ, 709, 791

Bontemps, S., Andre, P., Terebey, S., \& Cabrit, S. 1996, A\&A, 311, 858

Brunt, C. M. 2010, A\&A, 513, A67

Chandler, C. J., Terebey, S., Barsony, M., et al. 1996, ApJ, 471, 308

Churchwell, E., Povich, M. S., Allen, D., et al. 2006, ApJ, 649, 759

Churchwell, E., Watson, D. F., Povich, M. S., et al. 2007, ApJ, 670, 428

Davis, C. J., Chrysostomou, A., Hatchell, J., et al. 2010, MNRAS, 405, 759

Deharveng, L., Schuller, F., Anderson, L. D., et al. 2010, A\&A, 523, A6

Franco, J. 1983, ApJ, 264, 508

Goldsmith, P. F., Heyer, M., Narayanan, G., et al. 2008, ApJ, 680, 428

Goldsmith, P. F., Snell, R. L., Hemeon-Heyer, M., \& Langer, W. D. 1984, ApJ, 286, 599

Hennebelle, P., \& Falgarone, E. 2012, A\&ARv, 20, 55

Heyer, M. H., \& Brunt, C. M. 2004, ApJL, 615, L45

Heyer, M. H., Morgan, J., Schloerb, F. P., Snell, R. L., \& Goldsmith, P. F. 1992, ApJL, 395, L99

Heyer, M. H., Snell, R. L., Goldsmith, P. F., et al. 1987, ApJ, 321, 370

Hogerheijde, M. R., van Dishoeck, E. F., Blake, G. A., \& van Langevelde, H. J. 1998, ApJ, 502, 315

Klessen, R. S., \& Hennebelle, P. 2010, A\&A, 520, A17

Kwan, J., \& Scoville, N. 1976, ApJL, 210, L39

Lada, C. J. 1985, ARA\&A, 23, 267

Lada, C. J., \& Harvey, P. M. 1981, ApJ, 245, 58

Lamers, H. J. G. L. M., \& Cassinelli, J. P. 1999, Introduction to Stellar Winds (Cambridge: Cambridge Univ. Press)

Langer, W. D., \& Penzias, A. A. 1993, ApJ, 408, 539

Larson, R. B. 1981, MNRAS, 194, 809

Lichten, S. M. 1982, ApJL, 255, L119

Mac Low, M.-M. 1999, ApJ, 524, 169

Makovoz, D., \& Marleau, F. R. 2005, PASP, 117, 1113

Margulis, M., \& Lada, C. J. 1985, ApJ, 299, 925

McKee, C. F. 1989, ApJ, 345, 782

McKee, C. F., \& Ostriker, E. C. 2007, ARA\&A, 45, 565

Mitchell, G. F., Hasegawa, T. I., Dent, W. R. F., \& Matthews, H. E. 1994 ApJL, 436, L177

Moriarty-Schieven, G. H., Wannier, P. G., Tamura, M., \& Keene, J. 1992, ApJ, 400, 260

Myers, P. C., Heyer, M., \& Snell, R. L. 1988, ApJ, 324, 907 
Nakamura, F., \& Li, Z.-Y. 2007, ApJ, 662, 395

Nakamura, F., Kamada, Y., Kamazaki, T., et al. 2011a, ApJ, 726, 46

Nakamura, F., Sugitani, K., Shimajiri, Y., et al. 2011b, ApJ, 737, 56

Narayanan, G., Heyer, M. H., Brunt, C., et al. 2008, ApJS, 177, 341

Narayanan, G., Snell, R., \& Bemis, A. 2012, MNRAS, 425, 2641

Norman, C., \& Silk, J. 1980, ApJ, 238, 158

Ohashi, N., Hayashi, M., Ho, P. T. P., \& Momose, M. 1997a, ApJ, 475, 211

Ohashi, N., Hayashi, M., Ho, P. T. P., et al. 1997b, ApJ, 488, 317

Padgett, D., Noriega-Crespo, A., McCabe, C., et al. 2007, BAAS, 39, 750

Pineda, J. L., Goldsmith, P. F., Chapman, N., et al. 2010, ApJ, 721, 686

Qian, L., Li, D., \& Goldsmith, P. F. 2012, ApJ, 760, 147

Qian, L., Li, D., Offner, S., \& Pan, Z.-C. 2014, ApJ, submitted

Rebull, L. M., Koenig, X. P., Padgett, D. L., et al. 2011, ApJS, 196, 4

Rebull, L. M., Padgett, D. L., McCabe, C.-E., et al. 2010, ApJS, 186 , 259

Rieke, G. H., Young, E. T., Engelbracht, C. W., et al. 2004, ApJS, 154, 25

Shu, F. H., Adams, F. C., \& Lizano, S. 1987, ARA\&A, 25, 23

Snell, R. L., Loren, R. B., \& Plambeck, R. L. 1980, ApJL, 239, L17
Solomon, P. M., Huguenin, G. R., \& Scoville, N. Z. 1981, ApJ, 245, L19

Stojimirovic, I., Narayanan, G., \& Snell, R. L. 2007, ApJ, 660, 418

Tafalla, M., Santiago, J., Johnstone, D., et al. 2004, A\&A, 423, L21

Tamura, M., Ohashi, N., Hirano, N., Itoh, Y., \& Moriarty-Schieven, G. H. 1996, AJ, 112, 2076

Tennyson, J. 2005, Astronomical Spectroscopy: An Introduction to the Atomic and Molecular Physics of Astronomical Spectra (London: Imperial College Press)

Terebey, S., Beichman, C. A., Gautier, T. N., et al. 1990, ApJL, 362, L63

Torres, R. M., Loinard, L., Mioduszewski, A. J., \& Rodríguez, L. F. 2009, ApJ, 698,242

Ungerechts, H., \& Thaddeus, P. 1987, ApJS, 63, 645

Wang, P., Li, Z.-Y., Abel, T., \& Nakamura, F. 2010, ApJ, 709, 27

Wilson, T. L., Rohlfs, K., \& Hüttemeister, S. 2013, Tools of Radio Astronomy (Berlin: Springer)

Wu, Y., Wei, Y., Zhao, M., et al. 2004, A\&A, 426, 503

Wu, Y., Zhang, Q., Chen, H., et al. 2005, AJ, 129, 330

Wu, Y., Zhou, S., \& Evans, N. J., II 1992, A\&A, 394, 196

Zhou, S., Evans, N. J., II, \& Wang, Y. 1996, ApJ, 466, 296 